\title{
Cortactin Phosphorylation by Casein Kinase 2 Regulates Actin Related Protein 2/3 Complex Activity and Invadopodia Function
}

\author{
Steven Michael Markwell \\ West Virginia University, smarkwell@mix.wvu.edu
}

Follow this and additional works at: https://researchrepository.wvu.edu/etd

Part of the Biological Phenomena, Cell Phenomena, and Immunity Commons, Cancer Biology

Commons, and the Otorhinolaryngologic Diseases Commons

\section{Recommended Citation}

Markwell, Steven Michael, "Cortactin Phosphorylation by Casein Kinase 2 Regulates Actin Related Protein 2/3 Complex Activity and Invadopodia Function" (2018). Graduate Theses, Dissertations, and Problem Reports. 3685.

https://researchrepository.wvu.edu/etd/3685

This Dissertation is protected by copyright and/or related rights. It has been brought to you by the The Research Repository @ WVU with permission from the rights-holder(s). You are free to use this Dissertation in any way that is permitted by the copyright and related rights legislation that applies to your use. For other uses you must obtain permission from the rights-holder(s) directly, unless additional rights are indicated by a Creative Commons license in the record and/ or on the work itself. This Dissertation has been accepted for inclusion in WVU Graduate Theses, Dissertations, and Problem Reports collection by an authorized administrator of The Research Repository @ WVU.

For more information, please contact researchrepository@mail.wvu.edu. 


\title{
Cortactin Phosphorylation by Casein Kinase 2 Regulates Actin Related Protein 2/3 Complex Activity and Invadopodia Function
}

\author{
Steven Michael Markwell
}

Dissertation submitted to the School of Medicine at West Virginia University in partial fulfillment of the requirements for the degree of

\author{
Doctor of Philosophy in Cancer Cell Biology \\ Michael Schaller, Ph.D., Chair \\ Michael Ruppert, M.D./Ph.D. \\ Karen Martin, Ph.D. \\ Erik Bey, Ph.D. \\ William Petros, Pharm.D. \\ Scott Weed, Ph.D., Mentor \\ Cancer Cell Biology Program \\ Morgantown, West Virginia \\ 2018
}

Keywords: cortactin, Arp2/3 complex, CK2, invadopodia, Silmitasertib, HNSCC

Copyright 2018 Steven M. Markwell 


\begin{abstract}
Cortactin Phosphorylation by Casein Kinase 2 Regulates Actin Related Protein 2/3 Complex Activity and Invadopodia Function
\end{abstract}

Steven Michael Markwell

Malregulation of the actin cytoskeleton enhances tumor cell motility and invasion. The actinbinding protein cortactin facilitates branched actin network formation through activation of the actin-related protein (Arp) 2/3 complex. Arp2/3 complex activation is responsible for driving increased migration and extracellular matrix (ECM) degradation by governing invadopodia formation and activity. While cortactin-mediated activation of Arp2/3 complex and invadopodia regulation has been well established, signaling pathways responsible for governing cortactin binding to Arp $2 / 3$ are unknown. In this dissertation we identify casein kinase (CK) $2 \alpha$ phosphorylation of cortactin as a negative regulator of Arp2/3 binding. CK2 $\alpha$ directly phosphorylates cortactin at a conserved threonine (T24) adjacent to the canonical Arp2/3 binding motif. Phosphorylation of cortactin T24 by CK2 $\alpha$ impairs the ability of cortactin to bind Arp2/3 and activate actin nucleation. Decreased invadopodia activity is observed in HNSCC cells with expression of CK2 $\alpha$ phosphorylation-null cortactin mutants, shRNA-mediated CK2 $\alpha$ knockdown, and with the CK2 $\alpha$ inhibitor Silmitasertib. Silmitasertib inhibits HNSCC collective invasion in tumor spheroids and orthotopic tongue tumors in mice. Although overall cancer incidence rates are declining across the United States, the incidence of head and neck squamous cell carcinoma (HNSCC) continues to increase within the Appalachian region. To better understand the underlying factors leading to disproportionate outcomes, our group has established an Appalachian-specific HNSCC patient tissue cohort from surgically-resected tumors. This cohort represents all HNSCC stages, lesion types and morphologies, as well as cases that contain human papillomavirus (HPV) and/or tobacco and alcohol use. Moreover, we have generated several patient derived xenografts (PDXs) from these tissues, allowing further cellular, biochemical and preclinical therapeutic evaluation. Utilization of PDX tumors from this cohort will allow examination of critical steps in the development and potential treatment of invasive, metastatic, and recurrent Appalachian-associated disease. Matched patient and PDX sample availability enables personalized medicine and co-clinical trials aimed at reversing this Appalachian cancer health disparity and ultimately improving regional HNSCC patient care. 


\section{Dedication:}

I dedicate this to my fiancé Tiffany. I never would have accomplished this without your support, encouragement and confidence in my ability to succeed. You make me a better person than I ever could push myself to become. 


\section{Acknowledgements:}

Somehow, I finally made it through the maze that is graduate school. There have been many points where I thought about quitting and doing something else with my life but thanks to my many friends and family for dealing with me and convincing me that I really do enjoy the path I've chosen.

First, I would like to thank my fiancé Tiffany for dealing with all of my craziness and still being happy to share this journey with me. I never would have imagined that I'd meet the love of my life in Morgantown, but here we are. You've shown me what it means to be part of a team and I love you more every day. Thank you for being the foundation that allows me to be the best version of myself.

Next, I would like to thank my family. To my parents, Ken and Ronni; this accomplishment only possible due to your love and support. To my sister, Rae; thanks for being there to share in my frustrations and elations and letting me rant endlessly about the thing that's bothering me that day. To my brother, Dan; no matter what you find a way to put me in a better mood and that's priceless. To all my grandparents, Joan, Mort, Catherine, and Leslie; if not for all your hard work l'd never be in a position to pursue my intellectual curiosity. Lastly, to my cat Kahlan; thanks for all the snuggles and comfort when I'm worn out at the end of the day.

To my mentor, Dr. Scott Weed, thank you for righting the ship every time it seems like we're capsizing. You've shown me how to develop my own personal style while still conveying a professional attitude. Thank you for giving me the freedom to pursue my academic interests and for reigning me in when l've strayed too far. The skills l've learned under your guidance have set me up for a successful career.

Thank you to all my committee members, you have been incredibly helpful and constructive in shaping my understanding of the field. Your advice has been invaluable to reaching this point.

To the past and present members of the Weed lab; you have been incredible. To all the undergraduates and otolaryngology residents; thank you for bringing a unique point of view and enthusiasm to the lab. Jason, your ongoing advice has helped me avoid more pitfalls than I care to count. Karen, I managed not to burn down the lab after all. Mandy, thanks for always being there to help me when l've totally screwed up something and can't figure out what went wrong. Elyse, Friday afternoons aren't the same without wine, cheese and rock band. Brenen, for sharing a love of Game of Thrones and beer. It's going to be odd finishing the series on my own. Jessica, I'll miss our book sale and coffee weekends.

To all my Morgantown friends, even those who no longer call it home; thanks for turning this small college town into a home. In particular, Chris, Lindsay, Dan, Joe, and Jake; there was never a dull moment. Thanks for getting me through everything and making the good times roll. To the members of Tree of Life Congregation; thanks for taking in the stranger among you and treating them like one of your own. Margalit, your assistance and support have been a blessing.

Lastly, I would like to thank the Cancer Cell Biology program and West Virginia University for the opportunity to earn my Ph.D. You have shaped me into a capable scientist that I hope you will be proud to count among your alumni.

I'd like to summarize this experience in the words of Harry Caray, "Holy Cow!" 


\section{Table of Contents}

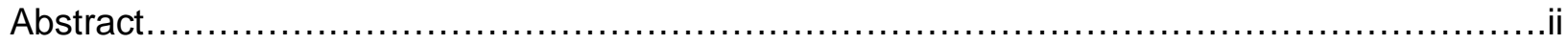

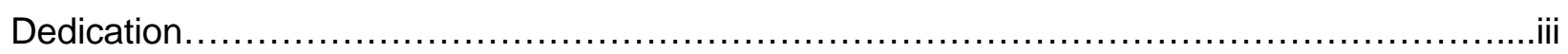

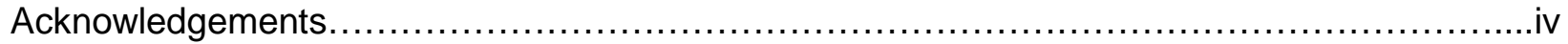

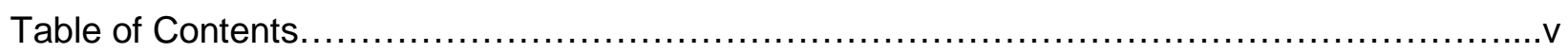

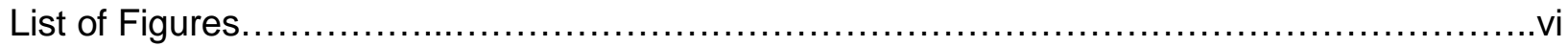

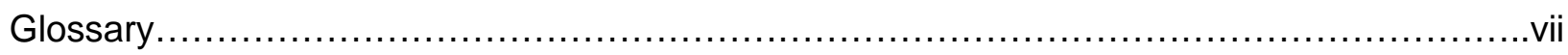

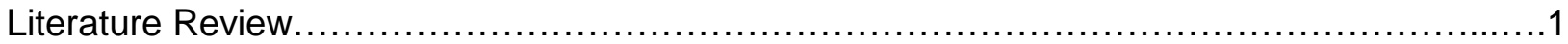

Head and Neck Squamous Cell Carcinoma

Mechanisms of Cancer Cell Invasion

Invadopodia

Cortactin

CK2

Patient-Derived Xenografts

Study 1: Cortactin Phosphorylation by Casein Kinase 2 Regulates Actin-Related Protein

2/3 Complex Activity, Invadopodia Function, and Tumor Cell Invasion..................55

Study 2: Establishment of an Appalachian Head and Neck Squamous Cell Carcinoma

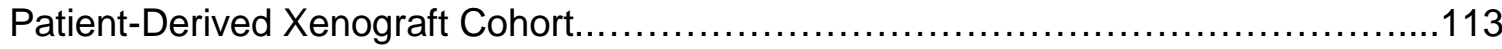

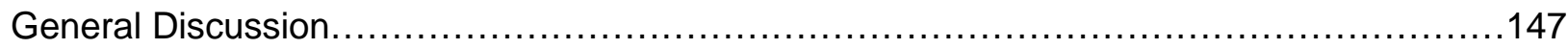

Appendix

Quantitative Measurement of Invadopodia-mediated Extracellular Matrix

Proteolysis in Single and Multicellular Contexts............................162

Review: Tumor vs. Stromal Contributions to Invasion in HNSCC ....................172

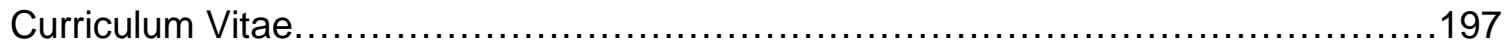




\section{List of Figures and Tables}

\section{Literature Review}

Figures

1. Tumor cell invasion modalities

2. Diagram representing cortactin functional domains

3. Cortactin Arp2/3 interaction modeling

4. CK2 $\alpha$ flexible regions

\section{Tables}

1. Immunocompromised mouse models

\section{Study 1}

Figures

Graphical Abstact

1. Cortactin T24 is required for binding and activation of Arp2/3 complex

2. T24 is required for cortactin-mediated invadopodia formation and ECM degradation in HNSCC cells

3. CK2 $\alpha$ phosphorylation of cortactin T24 inhibits Arp2/3 complex binding and activation

4. CK2 $\alpha$ is required for optimal HNSCC invadopodia function

5. Silmitasertib-mediated CK2 inhibition reduces invadopodia function in established HNSCC cell lines

6. Silmitasertib inhibits invadopodia function in HNSCC PDX cells

7. Silmitasertib impairs collective invasion in HNSCC

Supplementary Figures

1. T24 is required for optimal OSC19 invadopodia function

2. Effects of stable and transient cortactin knockdown on HNSCC invadopodia function

3. Silmitasertib inhibits CK2-dependent phosphorylation

4. Silmitasertib treatment does not impact collective invasive groups at the tumor invasive front

Supplementary Tables

1. Predictive analysis of human cortactin T24 phosphorylating kinases

\section{Study 2}

Figures

1. Representative examples of WVUSCC patient tumor anatomy and histology

2. Representative example of WVUSCC PDX implantation

3. WVUSCC PDX tumor monitoring and tissue passaging

4. Histological validation of WVUSCC PDX tumors

5. WVUSCC PDX-derived cell lines and gelatin degradation

\section{Tables}

1. WVUSCC patient cohort

2. WVUSCC tissue naming convention 


\section{Glossary}

\begin{tabular}{|c|c|}
\hline$\mu L$ & microliter \\
\hline$\mu \mathrm{m}$ & micrometer \\
\hline$\mu \mathrm{M}$ & micromolar \\
\hline 2D & two dimensional \\
\hline 3D & three dimensional \\
\hline A & alanine \\
\hline ABP & actin binding protein \\
\hline Arp & actin related protein \\
\hline ATP & adenosine triphosphate \\
\hline $\mathrm{AKT}(\mathrm{PKB})$ & protein kinase $\mathrm{B}$ \\
\hline BSA & bovine serum albumin \\
\hline C & Celsius \\
\hline CAFs & cancer associated fibroblasts \\
\hline CCND1 & cyclin D1 \\
\hline Cdc42 & cell division cycle 42 , GTP binding protein \\
\hline CK2 $2 \alpha$ & casein kinase $2 \alpha$ \\
\hline CMV & cytomegalovirus promoter \\
\hline $\mathrm{CP}$ & capping protein \\
\hline CORO & coronin \\
\hline c-Src & cellular sarcoma kinase \\
\hline Ctl & control \\
\hline CTTN & human cortactin \\
\hline Cttn & murine cortactin \\
\hline$\Delta$ & deleted \\
\hline $\mathrm{D}$ & aspartic acid \\
\hline DAPI & 4',6-diamidino-2-phenylindole \\
\hline DDW & aspartic acid-aspartic acid-tryptophan \\
\hline DMEM & Dulbecco's modified Eagle medium \\
\hline DMSO & dimethyl sulfoxide \\
\hline DNA & deoxyribonucleic acid \\
\hline DTT & dithiothreitol \\
\hline E- & epithelial \\
\hline ECM & extracellular matrix \\
\hline EDTA & ethylenediaminetetraacetic acid \\
\hline EMT & epithelial-mesenchymal transition \\
\hline ERK & extracellular signal regulating kinase \\
\hline FDA & Food and Drug Administration \\
\hline FFPE & formalin-fixed paraffin embedded \\
\hline F- & filamentous \\
\hline FBS & fetal bovine serum \\
\hline FITC & fluorescein isothiocyanate \\
\hline G- & globular \\
\hline GM-CSF & granulocyte-macrophage - colony stimulating factor \\
\hline GST & glutathione-S transferase \\
\hline$H \& E$ & hematoxylin and eosin \\
\hline $\mathrm{HCl}$ & hydrochloric acid \\
\hline HNSCC & head and neck squamous cell carcinoma \\
\hline HPV & human papilloma virus \\
\hline IF & immunofluorescence \\
\hline
\end{tabular}




\begin{tabular}{|c|c|}
\hline $\mathrm{IHC}$ & immunohistochemistry \\
\hline IL-3 & interleukin 3 \\
\hline KD & knockdown \\
\hline $\mathrm{kDa}$ & kiloDalton \\
\hline LB & luria broth \\
\hline $\mathrm{mL}$ & milliliter \\
\hline MMP & matrix metalloproteinase \\
\hline mRNA & messanger RNA \\
\hline $\mathrm{NaBH}_{4}$ & sodium borohydride \\
\hline NOD/SCIDy & non-obese diabetic/severe combined immunodeficiency \\
\hline NPF & nucleation promoting factor \\
\hline N.S. & not significant \\
\hline NTA & N-terminal acidic \\
\hline N-WASp & Neuronal-Wiskott-Aldrich Syndrome protein \\
\hline ODN & oligodeoxynucleotides \\
\hline OSC & oral squamous carcinoma \\
\hline p16 & cyclin-dependent kinase inhibitor 2A \\
\hline p53 & tumor suppressor protein 53 \\
\hline PBS & phosphate-buffered saline \\
\hline PCR & polymerase chain reaction \\
\hline PDX & patient-derived xenograft \\
\hline PP2A & protein phosphatase $2 \mathrm{~A}$ \\
\hline PRR & proline rich region \\
\hline $\mathrm{Rb}$ & retinoblastoma protein \\
\hline ROCK & Rho-associated serine/threonine kinase \\
\hline RNA & ribonucleic acid \\
\hline RNAi & ribonucleic acid interference \\
\hline ROI & region of interest \\
\hline RR & repeats region \\
\hline SCC & squamous cell carcinoma \\
\hline S & serine \\
\hline SCF & stem cell factor \\
\hline SDS-PAGE & sodium dodecyl sulfate polyacrylamide gel eletrophoresis \\
\hline $\mathrm{SH} 2$ & Src homology 2 \\
\hline $\mathrm{SH} 3$ & Src homology 3 \\
\hline shRNA & short hairpin ribonucleic acid \\
\hline SiRNA & small interfering ribonucleic acid \\
\hline Src & sarcoma kinase \\
\hline $\mathrm{SSH}$ & slingshot homolog phosphatase \\
\hline TAM & tumor-associated macrophage \\
\hline TIFF & tagged image file format \\
\hline VCA & verprolin, central, acidic \\
\hline W & tryptophan \\
\hline WASp & Wiskott-Aldrich Syndrome protein \\
\hline WAVE & WASp family verprolin-homologous protein \\
\hline WIP & WASp-interacting protein \\
\hline WT & wild-type \\
\hline Y & tyrosine \\
\hline
\end{tabular}




\section{Literature Review}

\section{Head and neck squamous cell carcinoma}

Head and neck squamous cell carcinoma (HNSCC) arises from epithelial cells lining the upper aerodigestive tract, encompassing the lips, oral cavity, nasopharynx, larynx, and hypopharynx $(1,2)$. Clinically HNSCC is further subdivided by anatomical location into disease of the oral cavity and pharynx or the larynx, based on differences in progression, response to therapy, and patient outcome $(1,2)$. Genetically HNSCC comprises two subtypes based on infection with human papilloma virus (HPV): HPV-positive and HPV-negative $(1,2)$. The largely positive prognosis associated with HPV-positivity in HNSCC resulted in the American Joint Committee on Cancer (AJCC) down-staging all HPV-positive disease effective January $1^{\text {st }}, 2017$ (3). Likewise, clinical trials have established dose de-escalation protocols for treatment of HPV-positive HNSCC (4). HPV-negative HNSCC is associated with prolonged tobacco and alcohol exposure $(1,2)$. As tobacco consumption in the United States declines, there has been a corollary decrease in HPVnegative HNSCC incidents (5). However, HPV-positive HNSCC has increased over this same time period, leading to an overall plateau in total HNSCC incidence over the past decade (5). Recent projections suggest that at the national level HPV-related HNSCC will become the predominant form of this cancer as early as 2020 (5).

While HPV-positive HNSCC accounts for nearly $20 \%$ of all HNSCC incidents, it is significantly enriched in oropharyngeal tumors where it is responsible for $60-80 \%$ of all HNSCC in this subregion (5-7). High-risk HPV subtypes 16 and 18 contribute to the vast majority of HPV-positive HNSCC (6-8). HPV is a circular double stranded DNA virus encoding 7 "early" stage proteins (E1-7) and 2 "late" stage proteins (L1 and L2) $(7,8)$. The early proteins E6 and E7 primarily target tumor suppressor genes p53 and Rb in host cells, transforming the infected epithelium and increasing susceptibility to oncogenic insult $(7,8)$. Despite impairment of these key tumor suppressor pathways, HPV-positive tumors have similar yet distinct mutational burdens as 
compared with HPV-negative tumors (9-11). Due to the influence of the other HPV encoded genes, these tumors tend to be poorly differentiated and highly metastatic to regional lymph nodes (12). Tumor differentiation is characterized by the invasive front, where the tumor interacts with the stroma. Well differentiated tumors show a clearly defined front, while poorly differentiated tumors show tumor cells intermixed with stromal cells making the exact tumor border difficult to discern (13). Moderately-differentiated tumors display either an intermediate phenotype or have regions along the tumor border than display both well and poorly differentiated segments (13). Conversely, HPV-negative HNSCC tumors tend to be well- to moderately-differentiated, invading as collective strands which cause immense loco-regional destruction of vital tissues leading to worse overall patient survival (12).

Further genomic analysis identified 5 distinct subtypes within HNSCC; two HPV-positive and three HPV-negative $(9,11)$. Characteristically, these tumors stratify into three supergroups: 1) the inflammation/mesenchymal group, 2) the basal group, and 3) the classical group. The basal supergroup is exclusively HPV-negative, while the other two can be further subdivided based on HPV status (9). The basal supergroup is enriched in hypoxia-responsive gene signatures and epithelial cell markers while the classical supergroup shows higher proliferation rates and highly correlates with tobacco consumption (9). The inflammation/mesenchymal supergroup exhibits an immune-responsive signature, enriched in $\mathrm{CD}^{+} \mathrm{T}$ lymphocyte infiltration and a poorly differentiated morphology (9). Despite similarities within supergroups, the HPV-positive tumors still display increased overall survival as compared to any of the HPV-negative subtypes (9). Another study further validated the distinct HPV-positive subtypes, again molecularly segregating the well differentiated tumors from the immune-responsive mesenchymal tumors (11).

While loco-regional disease is a hallmark of HNSCC progression, metastasis to distant organs such as the lungs, bone, brain, and liver have been noted $(14,15)$. Metastatic progression is a multi-step process during which tumors cell must breach the basement membrane, forge a path 
through the extracellular matrix $(E C M)$ to reach local vasculature or lymphatics, intravasate through the endothelium, extravasate into the secondary tissue, and engraft at the metastatic niche. Invasive carcinoma cells utilize an array of modalities in order to take advantage of cues from the tumor microenvironment, as well as internal aberrant signal transduction to accomplish these steps (16).

\section{Mechanisms of cancer cell invasion}

The inherent invasive tumor cell phenotype, along with its particular microenvironment, requires differing modes of cellular invasion as single cells or as collective invasive groups (Figure 1) $(17,18)$. Single-cell invasion can be subdivided into two modes. Non-proteolytic amoeboid motility driven through membrane blebbing allows cells to squeeze through pores in the ECM. Proteolytic mesenchymal invasion requires stromal cells or cells that have undergone epithelialmesenchymal transition (EMT) to degrade basement membrane and ECM components in order to generate permissive invasion pathways (18). Amoeboid invasion can be further characterized based on intracellular signaling involving specific Ras-family small GTPases of either the RhoRho-associated protein kinase (ROCK) pathway or the Rac pathway (19-24). Rho-dependent signaling results in actino-myosin contractility, compressing the cell and propelling the trailing edge of the cell forward $(20,23,24)$. At the leading edge of the cell, Rac signaling produces filopodial extensions, lamellipodia and podosomes driving the membrane forward and attaching to the ECM. Lamellipodia formation in response to extracellular growth factors, such as EGF, initiate membrane protrusion $(25,26)$. Podosomes anchor the actin cytoskeleton to the ECM allowing contractility at the trailing edge to push the cell forward $(19,23,24)$. Mesenchymal invasion relies almost entirely on the Rac-based signaling for directed cell motility $(18,19,24)$. Unlike amoeboid invasion, mesenchymal cells produce invadopodia, membrane protrusions specialized in proteolytic ECM cleavage through localization and secretion of specific matrix metalloproteinases (MMPs) (17,27-29). Both single cell modalities can give rise to multi-cellular 
streaming in which cells form loose transient cell-cell adhesions or simply take advantage of the cleared "tracks" left behind by previous invasive cells. It has also been noted that stromal cells can be involved in these multi-cellular streams, particularly when cancer-associated fibroblasts (CAFs) are recruited to "trail-blaze" a pathway through the ECM (30-32).

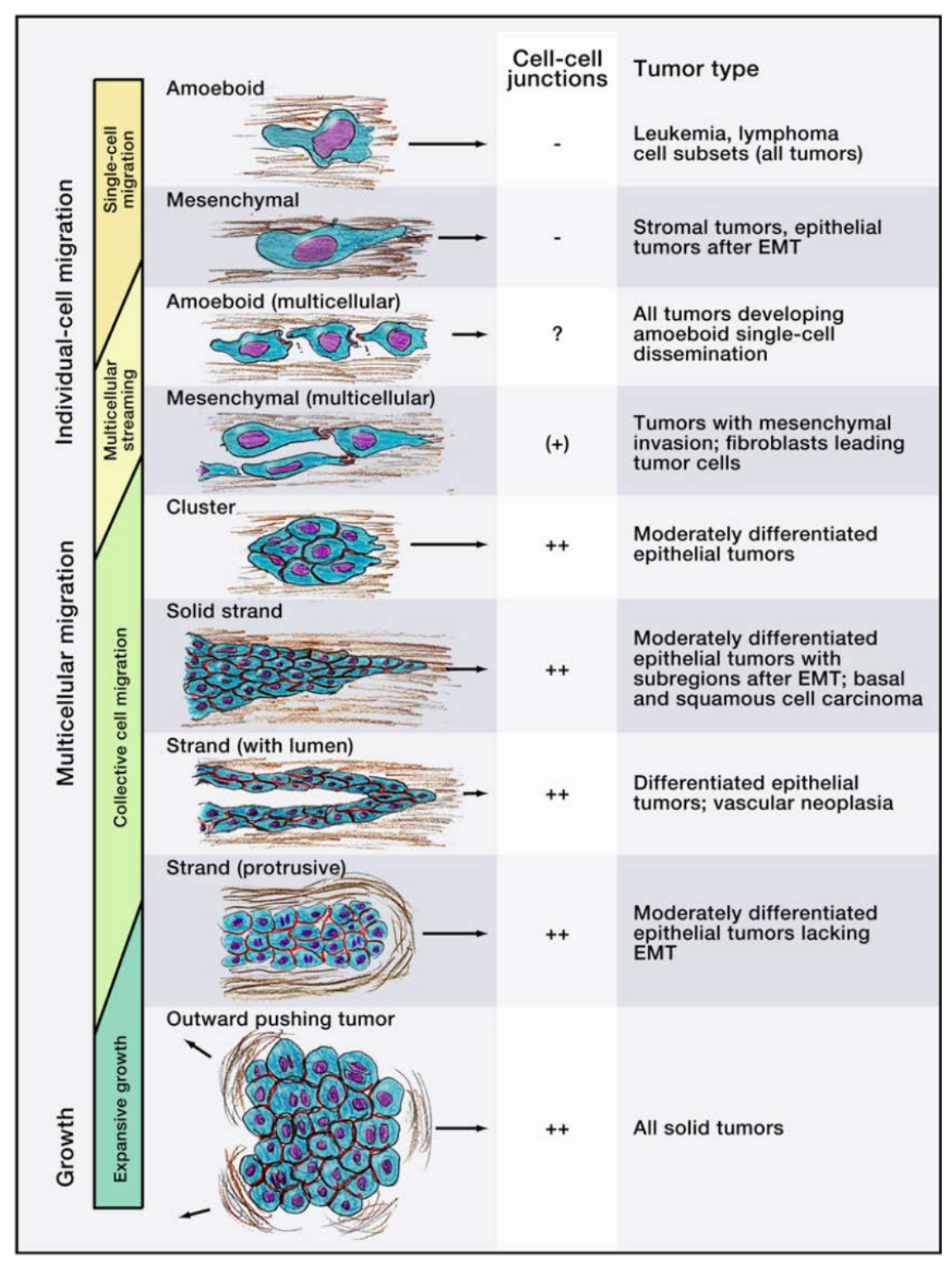

Figure 1. Tumor cell invasion modalities. Tumor cells utilize a spectrum of invasive modalities depending on the cell-cell interactions, tumor cell morphology and surrounding ECM.

Adapted from Figure 2 in Friedl, P. and Alexander, S. (2011) Cell 147 (5), p.992-1009.
Collective cellular

invasion is classified into

four main subtypes

based on morphologic

differences in the

collective groups arising

from differences

inherent to the invasive

cell and the surrounding

tissue: 1) cluster-like

invasion, 2) solid strand

invasion, 3) lumen-

containing strand

invasion, 4) protrusive

strand invasion $(17,18)$.

All collective invasion

relies upon cell-cell

adhesions between

either tumor cells

themselves or between 
tumor cells and infiltrating stromal cells within the tumor microenvironment $(17,18,33)$. Clusterlike invasion occurs when a compact "ball" of cells breaks away from the main tumor mass and invades as a tight group of epithelial cells $(17,18)$. Strand-like invasion can take on several morphologies based on cell morphology near the leading edge and is dependent upon maintaining polarity within the invasive group $(17,18)$. Solid strand collective invasion involves mesenchymal cells localizing to the leading edge of the strand, either through recruitment of stromal cells such as CAFs or from subregional EMT of tumor cells termed "tip" cells $(18,30,31)$. These tip cells maintain some expression of epithelial cell surface markers while maintaining junctions with the rest of the invasive strand to allow force transduction down the length of the collective group. Based on cell type and polarity, some invasive strands maintain a clear luminal region at the center of the strand, typically only exhibited in well-differentiated tumors $(17,18)$. In the absence of EMT or recruitment of mesenchymal stromal cells, collective strands display a rounded epithelial morphology where the collective mass extends a multi-cellular protrusion into the surrounding stroma $(17,18)$. At the extreme end of the collective invasion spectrum lies expansive growth-driven invasion wherein accelerated tumor cell proliferation (as compared to the surrounding tissue) generates an outward facing force that allows for pushing expansion of the invasive front $(17,18)$. The extent of this expansive growth depends on the rigidity of the surrounding tissue and can lead to extracapsular spread when metastatic lymph nodes can no longer contain the expanding tumor mass $(17,18)$. Tumor cells are typically capable of undergoing several if not all of these collective invasion modalities, where it is not uncommon to observe multiple differing modes within a single tumor or within a patient with multiple tumors (17). Therefore, a deeper understanding of the underlying mechanisms giving rise to these different modes of invasion is needed in order to better target and prevent invasive spread and improve patient outcomes. 
Rearrangement and organization of the actin cytoskeleton is necessary for formation of various cellular structures involved in cellular migration and invasion, as well as the transduction of forces through multi-cellular tumor cell groups. Migratory cells generate several actin-rich subcellular structures including podosomes, filopodia, lamellipodia and invadopodia (34-36). Formation and maturation of each of these structures requires tightly regulated cycles of actin polymerization and depolymerization through a variety of actin-binding proteins (ABPs) $(35,36)$. These proteins are key modulators of filamentous (F-) actin production, branching, stabilization, and eventual turnover and recycling of globular (G-) actin for further cycles of F-actin assembly $(26,37)$. F-actin is formed through ATP-bound G-actin monomer polymerization which rapidly adds to the "barbed' end of the filament (38). As the filament ages, ATP hydrolysis occurs rending ADP-bound actin towards the "pointed" end of the filament, leading to spontaneous depolymerization in the absence of filament-stabilizing ABPs (38). Specific ABPs enable complex structures to arise from F-actin, allowing for the formation of branches, bundles, and sheets dependent upon the specific ABPs involved (26,35-37). Filopodia contain mostly bundled actin strands, lamellipodia consist of mostly branched actin networks, while podosomes and invadopodia contain both branched actin near the base and bundled actin strands in the membranous extensions $(35,36)$. F-actin assembly is highly dependent on actin-related protein (Arp) 2/3 complex-mediated nucleation activity enhanced through nucleation promoting factors (NPFs) containing either a verprolin, central, acidic (VCA) domain found in the Wiskott-Aldrich Syndrome protein (WASp) family or an $\mathrm{N}$-terminal acidic (NTA) domain found in the cortactin family $(35,36,38)$. The type of specialized membrane protrusion formed depends upon the microenvironment as well as intrinsic cellular signals. In a 2D environment, cells typically exhibit lamellipodia and filopodia at the leading edge of the cell which is sufficient to pull the cell along a layer of $\operatorname{ECM}(35,36)$. In a 3D environment, it becomes difficult to differentiate podosomes from invadopodia since both subcellular structures have nearly identical components (39-44). While distinct markers distinguishing podosomes from invadopodia are contested in the field, consensus opinion proposes that WASp and Grb2 are 
considered posodosome-specific, while Nck1 and Mena appear invadopodia-specific (29). 3D protrusions containing F-actin and associated proteins such as cortactin, $\beta 1$ integrin, MMP14, N-WASp, paxillin, and talin have been observed in macrophages, dendritic cells, lymphocytes, melanoma, fibrosarcoma and breast cancer cells $(43,45)$. Recent studies have observed similar structures in tumor and endothelial cells in vivo $(40,43,46,47)$.

\section{Invadopodia}

Invadopodia were first identified by Chen et al. and characterized as membrane protrusions with localized ECM proteolytic activity in oncogenically transformed cells (48). Invadopodia share many ABPs found in other membrane protrusions. Invadopodia also contain microtubules and associated stabilizing proteins with the necessary transport machinery required for localization and secretion of MMPs (49-52). Invadopodia formation occurs in discrete spatial-temporal stages: 1) precursor core initiation, 2) precursor stabilization and 3) invadopodia maturation $(29,53)$.

Invadopodia are formed in response to chemotactic and proliferative environmental cues such as epidermal growth factor receptor (EGFR) stimulation by epidermal growth factor (EGF) $(48,49,54-$ 56). Intracellular signals are transmitted through Ras family GTPases, Src tyrosine kinase, protein kinase $C(P K C)$, Erk1/2, and others. Core initiation begins with cortactin-mediated recruitment of Arp2/3 complex, N-WASp, and cofilin to preexisting F-actin filaments $(29,53,57,58)$.

Stabilization is achieved through Src-dependent Tks5 binding to phosphoinositol 3,4-bisphosphate $\left(\mathrm{PIP}_{2}\right)$ on the plasma membrane (58-60) along with recruitment of $\beta 1$ integrin to the precursor where it binds to the ECM and initiates maturation signaling $(29,53,61)$. $\beta 1$ integrin activation through ECM binding creates an Arg kinase binding site on $\beta 1$ integrin initiating assembly of a Mena-Arg-SHIP2 complex that generates additional $\mathrm{PIP}_{2}$, further adhering the precursor core to the membrane (61). Simultaneously, cofilin severs existing F-actin filaments near the membrane, generating new barbed ends (62-64). Src and/or Arg-dependent cortactin 
phosphorylation creates docking sites for Nck1, recruiting N-WASp and Arp2/3 complex to initiate actin polymerization at the membrane $(29,55,58-60,65-68)$. WIP, Nck, and N-WASp bind to Src or Abl/Arg tyrosine kinases which target key C-terminal residues on cortactin $(55,65,69,70)$. Phospho-cortactin then interacts with various SH2 domain containing proteins , Arp2/3 complex through the NTA domain, and F-actin through the repeats region $(R R)(71,72)$. This multi-protein complex facilitates Arp2/3-mediated branched actin network formation, providing protrusive force on the cellular membrane to initiation invadopodia elongation $(35,36,71,72)$.

Invadopodia maturation involves elongation through the combined action of the actin bundling protein fascin and straight-filament NPFs of the formin family $(52,73)$. Additional recruitment of MMP14 as well as exosomes containing MMPs secreted at the invadopodia tip promote focalized matrix degradation $(50,74-76)$. Microtubules and certain intermediate filaments arrive at nascent invadopodia and are also involved in the maturation process $(29,52,77,78)$. Regulation of F-actin barbed ends at invadopoida tips is achieved by the uncapping activity of formins and Ena/Vasp. This enables G-actin addition to filament barbed ends near the inner membrane face, directly contributing in invadopodial membrane protrusion $(52,73,79,80)$. Bundling of the F-actin network within invadopodia is achieved through the activity of fascin, $\alpha$-actinin, and caldesmon. The combined activity of these dual F-actin bundling proteins serves to organize F-actin filaments in to parallel arrays, stabilizing the F-actin invadopodia core (80). The adhesion proteins talin and paxillin localize at the invadopodia tip to connect the F-actin network to various integrins, forming a bridge between the actin cytoskeleton and the $\operatorname{ECM}(29,53,56,78,81-83)$.

Invadopodia maturation is a tightly regulated process, striking a balance between the ECM adhesion necessary for motility and the proteolysis required maintain directed cellular invasion (29). As noted above, focal ECM degradation occurs when MMP14 (also known as membrane-

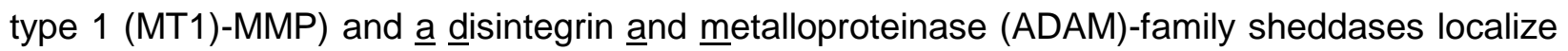
to invadopodia. These transmembrane proteases in turn act on the pro- forms of secreted 
extracellular MMP2 and MMP9, cleaving the pro- fragments to enable full enzymatic activity (51,57,84-86). MMP14 disruption through reduced expression, inhibition of activity or mislocalization profoundly diminishes invadopodia-mediated proteolysis and tumor cell invasion, highlighting a central role for this MMP in tumor invasion (87-92). MMP14 localization is regulated by plasma membrane dynamics, where recycling to the invadopodia tip can occur via clathrinmediated endocytosis or exocytic trafficking in a cortactin-dependent manner followed by exocyst complex-mediated sequestering at the membrane for rapid reincorporation at newly formed preinvadopodia $(49,76,89,91-96)$.

Although invadopodia were initially characterized in 2D cell culture, several 3D models demonstrate that these structures are not culture artifacts. Physiological in vivo and in vitro assays using native ECM have identified tumor cells that display invadopodia-like structures with $\mathrm{N}$ WASp, Tks5, cortactin, Src, MMP14 and Arp2/3 complex localization at sites of ECM proteolysis $(39,40,91,97-99)$. Furthermore, tumor cell lines that exhibit greater invadopodia function in 2D models almost universally display enhanced invasion capacity in 3D model systems. Similarly, podosomes have been found on osteoclasts, macrophages, smooth muscle and endothelial cells mediating homeostatic ECM reorganization (100-104). It is likely that tumor cells utilize this inherent cellular phenomenon in an attempt to pursue a more hospitable microenvironment as a result of increased primary tumor growth, resulting in depletion of local resources or by preventing adequate nutrient exchange due to incomplete angiogenesis and subsequent hypoxic conditions (105-107).

\section{Cortactin}

The ABP cortactin was originally identified as a Src kinase substrate that localized to peripherial F-actin $(108,109)$. Cortactin is a cytoplasmic protein ubiquitously expressed in all mammalian tissues of non-hematopoietic origin (71). Subsequent studies have elucidated roles in cell adhesion, migration, endocytosis, and invasion (71). Fittingly, cortactin localizes to various actin- 
rich membrane protrusions including lamellipodia, filopodia, podosomes, and invadopodia $(72,110)$. Cortactin is overexpressed in several invasive cancers including HNSCC, melanoma, breast, lung, bladder, colorectal, hepatocellular, and esophageal cancers (111-121). The CTTN gene that encodes cortactin is found on chromosome 11q13. Chromosome 11q13 amplification occurs in nearly $1 / 3$ of HNSCC patients and is responsible for a significant portion of cortactin overexpression events in this disease, though it is not a prerequisite for enhanced cortactin protein expression in all HNSCC patients (122-127). CTTN amplification is a poor prognostic marker, corresponding with increased lymph node involvement, distant metastasis, and decreased time to recurrence $(110,112,124,128-130)$. Regardless of the mechanism, overexpression of cortactin mRNA and/or protein corresponds with poor patient outcome in multiple patient cohorts $(119,125,131,132)$.

On the mechanistic level, cortactin is a multi-domain scaffolding protein that has been proposed to adopt several conformations. A closed "lollipop" auto-inhibitory conformation is thought to represent the inactive form, in which the Src homology $(\mathrm{SH}) 3$ domain folds back to bind to the proline-rich region (PRR). The active form is thought to assume an open "rod-like" conformation in which the molecule is linearized, allowing multi-protein complex formation $(109,133,134)$.

\section{\begin{tabular}{|l|l|l|l|l|l|l|l|l|l|l|} 
NTA & R1 & R2 & R3 & R4 & R5 & R6 & Helix & PRR & SH3 \\
\hline
\end{tabular}}

Figure 2. Diagram representing cortactin functional domains. NTA, N-terminal acidic domain; R1-R6, repeats regions; Helix, alpha helical domain, PRR, proline rich region; SH3, Src homology 3 domain.

Based on primary structure and functional analysis, cortactin has been subdivided into multiple domains/regions, including the NTA domain, the RR, an a-helical domain, the PRR, and an SH3 domain (Figure 2)(135). The NTA domain mediates binding to and activation of Arp2/3 complex via a highly conserved DDW motif common to most NPFs (72,109,110,136-143). Arp2/3 activation by cortactin alone is weak compared to WASp-family NPFs, requiring direct and/or indirect WASp-protein binding to cortactin to facilitate robust Arp2/3 complex activation 
$(72,110,137,144,145)$. Following the NTA domain, the F-actin interacting domain contains 6.537 amino acid tandem repeats. Binding to F-actin occurs at sequences around the $4^{\text {th }}$ repeat $(109,139)$. Lysine acetylation/deacetylation within the repeats region modulates F-actin binding efficiency. HDAC6-mediated cortactin deacetylation enhances F-actin binding, while a group of histone acetyltransferases including pCAF/p300 and SIRT1 acetylate cortactin to impair F-actin binding (146-148). The ability of cortactin to simultaneous bind Arp2/3 and F-actin stabilizes the overall F-actin network. This is achieved by cortactin NTA binding to Arp2/3 complex at the base of one actin filament, and the F-actin binding domain interacting directly with the side of a second F-actin filament. This stabilization effect is required for enhanced cell migration and tumor cell invasion $(71,72,146,149)$. The $\alpha$-helical domain contains a calpain cleavage site that when proteolyzed by calpain 2 , enhances cellular migration likely due to the inability of the cleaved product to adopt the closed conformation $(150,151)$. Both the PRR and SH3 domain are responsible for integrating upstream signaling events via post-translational modifications and mediating protein-protein interactions with other cortactin binding proteins $(66,70,128,137,152-$ 156).

The cortactin C-terminus contains the vast majority of its post-translational modification sites (157). Src and Abl family kinases phosphorylate tyrosine 421, 470, and 486 in the PRR, leading to enhanced tumor cell motility, invasion, and metastasis $(65,72,128,158)$. Tyrosine phosphorylated cortactin is enriched in lamellipodia, invadopodia, and dorsal waves (153). SH2 domain containing cortactin-binding proteins including Nck1, Crk, and Abl family kinases utilize these phospho-tyrosine residues dock with cortactin, ultimately recruiting other binding partners to form large multi-protein complexes $(65,66,158-160)$. The Nck1 SH2 domain binds pY421 and pY466 then recruits N-WASp whose PRR interacts with the cortactin SH3 domain $(65,158)$. This trimolecular complex enhances Arp2/3 complex nucleation activity through synergistic activities of the cortactin NTA and N-WASp VCA domains. Some controversy exists regarding the function 
of tyrosine phosphorylated cortactin on NPF activity. One study concluded that Src-dependent cortactin phosphorylation diminished N-WASp binding and Arp2/3 actin nucleation activity through maintaining a closed conformation (161), while another study found that cortactin tyrosine phosphorylation does not induce a closed conformation (158). How these various posttranslational modifications to C-terminal cortactin residues impact one another and ultimately how these signals integrate to control actin dynamics remains unresolved.

In order to increase ABP recycling, protein tyrosine phosphatase (PTP) 1B dephosphorylates cortactin tyrosine residues (162). The actin capping protein MenalNV protects cortactin from PTP1B-mediated dephosphorylation, though the exact mechanism remains unclear (163). Tyrosine dephosphorylation plays a major role in invadopodia regulation, as impaired phosphorylation at $\mathrm{Y} 421, \mathrm{Y} 466$, and $\mathrm{Y} 482$ significantly decreases invadopodia formation $(72,128,164)$. Additionally, FAK has been shown to modulate Src-dependent cortactin phosphorylation at Y421, Y470, and Y486, enhancing focal adhesion turnover and allowing for increased cell motility (128). Cortactin is also a substrate for a variety of serine/threonine kinases. Erk1/2 targets serine (S) 405 and 418; ㅁ21-ạctivated kinase Rac1/Cdc42 activated kinases (PAK) 1, 3, and 4 phosphorylate S405 and S418 as well as S113 in first repeat region $(128,152,153,165)$. Erk1/2-mediated phosphorylation of S405/418 enhances binding between cortactin SH3 domain and the N-WASp PRR by releasing cortactin from the auto-inhibited state. Erk phosphorylation of N-WASp causes a similar release of VCA autoinhibition. The newly formed complex between activated cortactin SH3 and N-WASp PRR domains promotes Arp2/3 nucleation activity, leading to increased lamellipodia persistence and subsequent enhanced motility in HNSCC cells (128,166-168). S113 phosphorylation appears to further regulate interaction between the RR and F-actin filaments (152). Other studies show that both Erk1/2 and PAK-dependent serine phosphorylation are required for optimal ECM degradation in melanoma cells (49). In addition, protein kinase D (PKD) phosphorylates cortactin S298 in the sixth repeat and S348 preceding the 
helical-PRR $(169,170)$. PKD phosphorylated cortactin shows impaired binding to $\beta$-catenin and vinculin in adherens junctions, uncoupling E-cadherin mediated cell-cell interactions from the actin cytoskeleton, resulting in junction dissolution (169-172). While these studies provide important mechanistic insights into cortactin regulation by phosphorylation, several phosphorylation sites identified by comprehensive mass spectrometry screens currently do not have assigned functions (157).

The cortactin $\mathrm{SH} 3$ domain mediates binding to a variety of PRR-containing cortactin binding partners. These interactions allow cortactin to integrate signals that control signal transduction (Fgd1, BPGAP1, Abl family kinases), actin polymerization (N-WASp, WIP), cell-cell and cell-ECM adhesions (ZO-1, FAK), endocytosis and exocytosis (ACK1, HIP1R, AMAP, Dynamin-2, SHANK2, CD2AP), and actomyosin contractility (myosin light chain kinase (MLCK)) $(71,128,134,173)$. These processes collectively play major roles in directed cellular chemotaxis and migration, as well as invadopodia function and ECM invasion.

While many studies have focused on modification and interactions with the cortactin C-terminal domain, the cortactin NTA domain that harbors the NPF activity centered around the DDW motif has also been extensively studied. Several studies have demonstrated that removal of the DDW motif significantly impairs binding to Arp $2 / 3$ complex and subsequently prevents invadopodia formation and maturation in a variety of cell lines $(137-143,174)$. NPFs enhance Arp2/3-mediated actin polymerization by stabilizing a conformation in which Arp2 and Arp3 subunits mimic an Factin barbed end, allowing for rapid assembly of a new actin filament $(137,140,175)$. The cortactin 

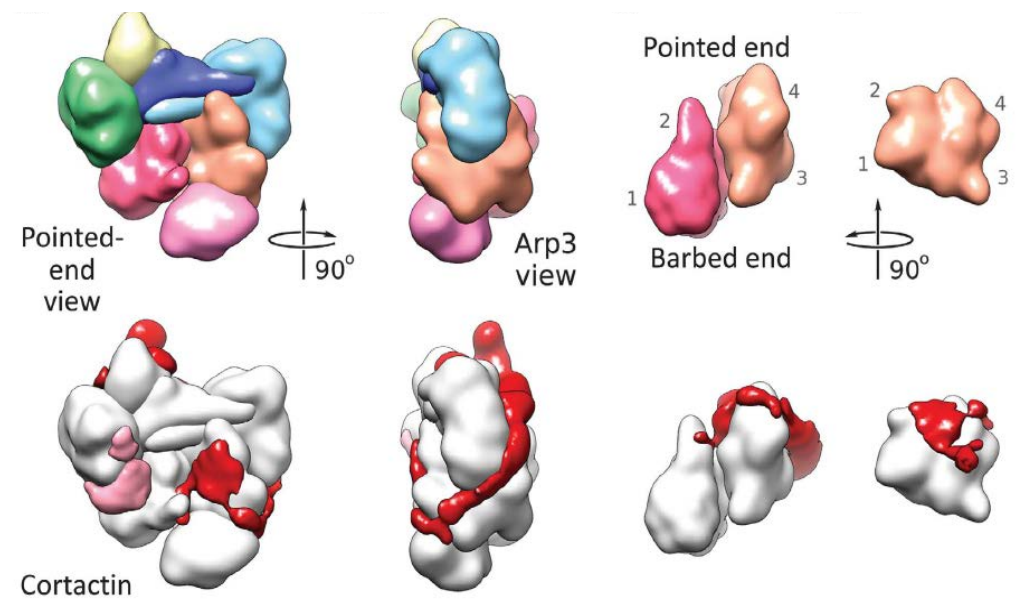

Figure 3. Cortactin Arp2/3 interaction modeling

3D reconstruction of cortactin bound Arp2/3 complex reveals cortactin NTA domain bridging the pointed end mimic. Panels display views of Arp2/3 complex from the pointed end (left) and Arp3 (left center) as well as an Arp2\&3 dimer from the side (right center) and Arp3 alone (right). Arp2/3 complex is shown in the top row with color subunits. On the bottom, Arp2/3 complex is colored white and cortactin is shown in red.

Adapted from Figure 3 in $X u, X$. et al. (2012) EMBO, 31(1), p.236-247.
NTA domain directly binds both Arp2 and Arp3 along the surface opposite to the barded end mimic, pinching the two subunits together in order to stabilize the active Arp2/3 complex conformation (38,175). Similarly, NPFs from proteins with VCA domains bridge between Arp2 and Arp3 along the pointed end of the complex, stabilizing Arp2/3 complex in a manner conducive to $\mathrm{G}-$

actin binding (175). Since the VCA domain is also capable of binding G-actin, these NPFs demonstrate a greater ability to activate Arp2/3 complex, enhancing the nucleation rate. Mechanistic studies to determine the spatial and temporal coordination of cortactin and WASpfamily NPFs in Arp2/3-mediated branch point assembly have yielded differing mechanisms. One proposed mechanism involves simultaneous binding of N-WASp and cortactin to Arp2/3 complex (137). A second mechanism proposes that N-WASp binding and activating Arp2/3 complex prior to displacement by cortactin. In this mechanism, cortactin then binds to and stabilizes Arp2/3 complex free of N-WASp while anchoring the newly formed "daughter" filament to the existing "mother" filament via the RR (176). Further work is required to fully elucidate the precise mechanisms utilized by cells in regulating motile and invasive behavior reliant on Arp2/3 activity, as well as how upstream signaling events alter NPF binding and activation during these cellular events. 
Cortactin is a key component of the actin-based invadopodia machinery and as such is recognized as the canonical invadopodia marker $(71,72,128)$. Initial work demonstrated that cortactin formed a complex with paxillin and PKC within invadopodia that correlated with sites of ECM degradation (72). Likewise, cortactin expression and subcellular localization correlates with invadopodia formation and maturation in transformed fibroblasts and multiple invasive cancer cell lines $(71,72)$. Mechanistically, cortactin initiates invadopodia formation through recruitment to sites of actin polymerization and ECM adhesion giving rise to immature pre-invadopodia $(139,141,142)$. Subsequently, other invadopodia-associated proteins including Arp2/3 complex, N-WASp, and Tks5, are recruited to enhance and maintain Arp2/3 activity in order to drive membrane protrusion. Invadopodia maturation is characterized by subsequent recruitment and localization of MMPs, particularly MMP14, to sites of focalized ECM degradation $(74,76,87,89,91,95)$. One model suggests the following order of events for cortactin-mediated invadopodia initiation (176). First, non-phosphorylated cortactin arrives at an actin filament, then recruits N-WASp, Arp2/3 complex, and cofilin. Cortactin tyrosine phosphorylation releases cofilin to begin severing F-actin, generating a localized increase in free barbed ends, and clearing a docking site for Nck1 binding to cortactin. Nck1 recruits N-WASp into the trimolecular complex described previously. Lastly, PTP1B dephosphorylates cortactin, releasing Nck1 and N-WASp and inhibiting cofilin in order to stabilize the newly formed actin network. Invadopodia turnover is necessary for efficient tumor cell invasion $(177,178)$. Two mechanisms have been elucidated for driving invadopodia disassembly, one involving Rac1-Trio-PAK1 signaling cascade and another through RhoG activity. In the first pathway Trio, a Rac1 guanine exchange factor (GEF), activates Rac1 which in turn activates PAK1 or PAK4 $(165,178,179)$. PAK-dependent phosphorylation of cortactin S113, S150 and S282 destabilizes invadopodia resulting in disassembly presumably through decreased interaction between the cortactin RR and F-actin $(152,178,179)$. The second pathway involves RhoG-dependent paxillin Y31 and Y118 phosphorylation leading to calpain activation $(180,181)$. Calpain cleaves several ABPs including cortactin, talin, and WASp 
destabilizing the invadopodia F-actin core leading to invadopodia disassembly $(150,151,181-$ 183).

In addition to forming invadopodia actin networks, cortactin is also involved in endocytic transport and secretion of various MMP cargoes at invadopodia. During endocytosis, the cortactin SH3 domain binds dynamin-2, linking the cortical actin cytoskeleton to the membrane severing machinery around clathrin-coated vesicles (155,184-187). De novo cortactin-mediated actin network formation initiates membrane invagination prior to membrane severing through dynamin-2 (155,184-187). Following vesicle formation, cortactin and Arp2/3 complex remain attached to a subset of vesicles where further actin polymerization propels newly formed vesicles through the cytoplasm (188). RNAi-mediated cortactin reduction dramatically reduces MMP2 and MMP9 secretion as well as MMP14 localization to invadopodia tips. Conversely, overexpression of cortactin corresponds with increased MMP secretion $(189,190)$.

\section{CK2}

CK2, previously misnamed casein kinase-2, is a ubiquitously expressed, highly conserved, constitutively active serine/threonine kinase consisting of two catalytic subunits ( $\alpha$ or $\left.\alpha^{\prime}\right)$ and two $\beta$ regulatory subunits (191-193). The CK2 holoenzyme exists as a heterotetrameric complex or as lone catalytic subunits in cells. The complex is exclusively cytoplasmic, while the catalytic subunits can be found in both the cytoplasm and nucleus (191,192,194-197). The regulatory subunits play a role in substrate recognition as well as modulating catalytic function of the holoenzyme $(192,193,196,198,199)$. This is dissimilar to most heterotrimeric kinases in which the regulatory subunit serves as a molecular switch, activating the catalytic subunit's kinase activity. Unlike most kinases, the CK2 catalytic subunit can facilitate phosphorylation utilizing either ATP or GTP hydrolysis $(191,195,198,200)$. CK2 $\alpha$ contains two lobular domains with the catalytic site residing at the interface between the domains $(201,202)$. The constitutive activity arises from a unique structure, leaving the activation segment and the third a helix (termed $\alpha \mathrm{C}$ ) permanently in 


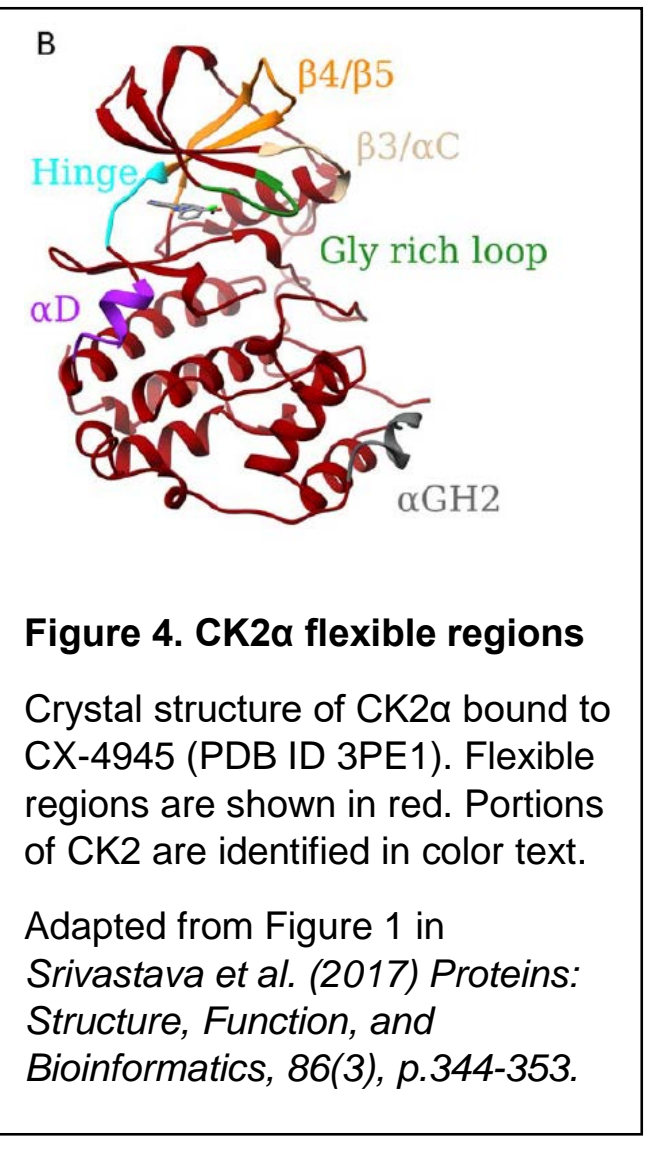

the active conformation $(201,202)$. Additionally, human CK2 $\alpha$ subunits exhibit remarkable conformational heterogeneity even when compared to evolutionarily similar orthologs which remain sequentially and structurally similar (202). One recent study identified 5 distinct regions of conformational flexibility, a glycine-rich $p$-loop, the $\beta 3 / \alpha C$ loop, the $\beta 4-\beta 5$ region, the Hinge/ $\alpha D$ region, and the $\alpha \mathrm{GH} 2$ helix (201). The $\beta 4-\beta 5$ region mediates binding to the regulatory subunit, while the glycine-rich $\mathrm{p}$-loop and the Hinge/aD region confer differences in access to the ATP binding pocket, therein representing "closed" vs. "open" conformations (201). As of yet, no functional role has been elucidated for the $\beta 3 / \alpha C$ loop or the $\alpha \mathrm{GH} 2$ helix. Due to its very general

recognition motif (S-X-X-E/D/pS/pT) CK2 is recognized as one of the most promiscuous kinases in the genome with over 300 proposed substrates and more than 700 predicted CK2 phosphosites matching its recognition motif in the PhosphoSitePlus dataset $(191,194)$. With the advent of CRISPR/Cas9-mediated knockout technology, a recent study attempted to verify substrate specificity for proposed CK2 targets, confirming 48 catalytic subunit regulated phospho-sites and another 163 heterotetrameric complex regulated phospho-sites in C2C12 mouse myoblast cells (194).

Despite its constitutive activity, CK2 appears to be regulated at the protein level rather than transcriptionally $(203,204)$. Early studies reveal intracellular shuttling between the nucleus and cytoplasm and subcellular localization as the major regulatory mechanism in CK2-dependent signaling (196,205-208). While holoenzyme shuttling between the nuclear and cytoplasm 
appears to be commonplace in normal cells, subunit specific responses have been noted in various cancer cell models where nuclear CK2 is associated with a pro-survival phenotype $(203,207,209,210)$. One study found that Bcr-Abl mediated-CK2 $\alpha$ phosphorylation suppresses CK2 activity, which can reversed with PTP1B, demonstrating a post-translational regulatory mechanism of CK2-mediated signaling networks (211).

CK2 is critical for maintaining cellular homeostasis, as minor changes in expression or nuclear localization have robust effects on proliferation and cell viability $(203,207,208,210,212-214)$. Initial studies on CK2 signaling demonstrated enhanced activity and nuclear localization in response to androgenic growth stimuli, whereas androgen depletion or decreased CK2 expression induced apoptosis $(205,212,214,215)$. Additionally, CK2 modulates many canonical cell proliferation signaling networks including the Wnt, EGFR, Ras, AKT, Hedgehog and JAK/STAT pathways (216-220). CK2 mediates crosstalk between the Wnt and EGFR pathways via Erk2-dependent CK2 phosphorylation. This results in inhibiting a-catenin phosphorylation and promotes subsequent $\beta$-catenin translocation to the nucleus that drives glioblastoma invasion (216). In some cases CK2 is required for downstream signal transduction, as demonstrated in Hedgehog signaling where CK2 phosphorylates Smoothened and stabilizes Cubitus interruptus (Ci) and JAK/STAT signaling necessary for JAK2-mediated STAT activation that is required for expression of suppressor of cytokine signaling (SOCS)-3, both of which are necessary for tissue homeostasis and repair as well as maintaining local stem cell populations $(219,220)$. In other cases CK2 merely enhances oncogenically driven proliferation as seen in H-Ras and AKT models $(217,218)$. Likewise, CK2 mediates many cell death signals, particularly responses to DNA damage. CK2 directly modulates the DNA damage response pathway, phosphorylating BRCA1 C-terminal residues that are notably mutated in breast cancer to disable a key checkpoint in DNA repair (221). Indirectly CK2 modulates the DNA damage response by phosphorylating several tumor suppressor proteins including p53, structure-specific recognition protein (SSRP)1, 
promyelocytic leukemia gene (PML), and PTEN. This alters transcription and replication activity within the nucleus (222-226). CK2 $\alpha$ suppresses apoptotic signaling via a multitude of posttranslational modifications to apoptotic machinery including the PI3K/AKT pathway, survivin, various inhibitors of apoptosis proteins (IAPs), caspases, the $\mathrm{Bcl} 2$ pathway, and the reactive oxygen species response pathway $(195,227)$. As further evidence of the pro-survival role of CK2, impairment of CK2 signaling, either through removal of either CK2 $\alpha$ or CK2 $\beta$ in mice or simultaneous removal of CK2 $\alpha$ and CK2 $\alpha^{\prime}$ in yeast is lethal $(192,195)$. The fine-tuning between pro and anti-survival signaling is best demonstrated in retinal astrocytes, where CK2 expression is necessary for neo-angiogenesis during development, while extended CK2 signaling ultimately results in retinopathy $(228,229)$.

CK2 $\beta$ is necessary for localizing the holoenzyme to the plasma membrane, where it phosphorylates several ABPs including spectrin, ankyrin and adducin $(200,230)$. Important to this Dissertation, CK2 phosphorylates the cortactin homologue HS1 at an unidentified site in the comparable NTA region, as well as residues near the DDW region in the Arp2/3 regulatory proteins N-WASP and WAVE2 (231-235). There is conflicting evidence about the nature of CK2mediated phosphorylation surrounding the DDW motif in WASp family proteins. Phosphorylation of the DDW flanking residues in WAVE/SCAR proteins impairs their ability to act as NPFs $(233,236)$. Similarly, CK2-mediated phosphorylation impairs N-WASp activity while simultaneously enhancing clathrin-mediated EGFR endocytosis (235). Contrary to these finding, another study demonstrated that WASp phosphorylation by CK2 enhanced binding to and activation of Arp2/3 complex (234). Work in this Dissertation indicates that CK2-dependent cortactin NTA phosphorylation impairs binding to and activation of Arp2/3 complex (237). In addition to modulating actin dynamics, CK2 also phosphorylates many microtubule associated proteins including microtubule-associated protein (MAP)-1A, MAP-1B, MAP2, MAPT, $\beta$-tubulin, stathmin, and dynein. This is congruent with association of CK2 with the mitotic spindle in dividing 
cells $(196,197,238-244)$. Collectively these data demonstrate a role for CK2 signaling having a direct impact on the actin and tubulin cytoskeletons involved in cellular motility, mitosis, and cytokinesis.

Given that CK2 influences many prominent pathways utilized during tumor initiation and progression, it is not surprising that CK2 is overexpressed in a multitude of different cancer types (191,195,204,227,245-247). Increased tumor cell CK2 expression correlates with transformation, cell growth, cell cycle progression, cell survival, angiogenesis, and tumor cell motility, as well as decreased apoptosis and drug resistance $(191,195,203,227,245)$. One study identified CK2 among a 186-gene "invasiveness signature", where enhanced expression correlates with decreased overall and metastasis-free survival in medulloblastoma, lung, breast, and prostate cancers $(245,248)$. Specific to HNSCC, CK2 overexpression correlates with increased tumor grade, stage, and clinical outcome as well as radio- and chemotherapy resistance $(227,246,249-$ 252). CK2 is enriched at the invasive tumor front, primarily within tumor cells, cancer-associated fibroblasts, and tumor-infiltrating lymphocytes. This implies that CK2 overexpression plays important roles in tumor-associated inflammation, invasion, and metastatic progression $(251,253)$. With regards to directed cellular invasion, CK2 expression is critical for IL-6-driven tumor cell chemotaxis (254). In addition, CK2 activity further enhances a tumor stem cell phenotype and sphere formation, supporting an important a role in cancer progression and disease recurrence (255).

While CK2 is not an oncogene in the traditional sense, modest increases in CK2 expression enhance the tumorigenicity of other driver oncogenes in various transgenic mouse models $(195,247,256-259)$. In addition, CK2 directly phosphorylates several cancer-promoting transcription factors including NF-kB, p53, TAp73, and Twist to promote a pro-malignant phenotype $(227,246,247,254-256,258)$. CK2 also phosphorylates IKBa and RELA to promote IKK $\beta$ activity while simultaneously suppressing p53 expression at both the mRNA and protein 
levels $(227,246,247)$. One study found that transgenic CK2 overexpression in mice leads to loss of p53 expression, enhancing lymphoma onset (256). In a Tal-1-driven murine acute lymphoblastic leukemia (ALL) model, CK2 $\alpha$ co-overexpression enhanced tumorigenesis and significantly decreased overall survival (258). Other studies found that CK2 $\alpha$ overexpression enhances Notch1, C-Myc and Wnt pathway component expression while modulating NF-kB signaling, ultimately leading to tumorigenesis $(247,259,260)$. Conversely CK2 impairment decreases expression of $\beta$-catenin, c-Myc, CCND1, Bcl-XL, Integrins- $\alpha 3,-\alpha 6,-\beta 4$, VEGF, Slug, Snail, vimentin and Notch1 while increasing expression of p53,TAp63, TAp73 and E-cadherin $(227,247,255,259-261)$. CK2 $\alpha$ expression (but not CK2 $\beta$ ) is sufficient to confer drug resistance in acute myeloid leukemia (AML) and osteosarcoma models (203). Similarly, increased CK2 activity is induced in prostate cancer cell lines upon treatment with cytotoxic agents and correlates with resistance to apoptosis (214). Exogenous CK2 $\alpha$ expression alone is sufficient to abrogate drug-induced apoptosis in certain tumor cell lines $(210,214)$. Collectively these studies support a global role for CK2 in promoting and maintaining signaling necessary for oncogenic initiation and progression.

Given the myriad signal transduction pathways enhanced through CK2 activity, CK2 has garnered much interest as a drug target. This one target modulates cellular response to mitogenic signals, inflammatory cytokines, apoptosis and cell survival as well as drug efflux pump efficiency, angiogenic signaling, and tumor invasion and metastasis (195,203,227,229,247,262). Many preclinical models have shown anti-cancer benefits using antisense and siRNA targeted CK2 reduction $(195,213,227,261)$. Antisense oligodeoxynucleotides (ODN) against CK2 inhibits growth and induces apoptosis in prostate cancer and HNSCC cell lines along with associated xenografts $(212,213,227,252,263,264)$. Tenfibgen nanocapsule-mediated anti-CK2 ODN delivery exhibits high tumor specificity in vivo $(195,213,263,264)$. Reduced CK2 expression resultant from antisense ODN or RNAi treatment impaired HNSCC cell migration and invasion while 
simultaneously resensitizing HNSCC tumors and cell lines to cisplatin $(227,261)$. Furthermore, impaired CK2 signaling abrogates stem-like gene expression, clonal survival, and spheroid formation $(255,260)$.

While preclinical CK2 modulation studies continue to show promise, clinical trials targeting CK2 specific signaling have shown marginal benefit. Pharmacological CK2 inhibitors developed to date include apigenin, CIGB-300, 2-Dimethylamino-4,5,6,7-tetrabromobenzimidazole (DMAT), 5,6dichloro-1- $\beta$-D-ribofuranosylbenzimidazole (DRB), emodin, hematein, Silmitasertib (also known as $\quad$ CX-4945), 4,5,6,7-tetrabromobenzotriazole (TBB), quinalizarin, (E)-3-(2,3,4,5tetrabromophenyl)acrylic acid (TBCA), and 6,7-dichloro-1,4-dihydro-8-hydroxy-4-[(4methylphenylamino)methylen]dibenzo[b,d]furan-3(2H)-one (TF). These compounds display varying degrees of anti-tumor efficacy and enzyme specificity (191,262,264-276). Nearly all of these CK2-targeted inhibitors are competitive ATP/GTP small molecules, inherently leading to poor target specificity due to the inherent conservation of this pocket in the active sites of kinases $(262,277,278)$. CK2 pharmacological inhibition decreases proliferation in breast carcinoma and lymphoma cell lines $(247,259,279)$. Although CK2 inhibition as a single agent has shown limited success, combination therapies with CK2 inhibitors show improved efficacy. One such study demonstrated that CK2 inhibition in Imatinib insensitive Bcr-Abl-driven ALL reduced cell viability and resensitized the surviving cell population to Imatinib treatment (279). Similarly, increased apoptosis was found in prostate and colorectal carcinoma using CK2 inhibitors with doxorubicin (278). CK2 inhibition also sensitizes colorectal carcinoma and rhabdomyosarcoma cells to TNFrelated apoptosis-inducing ligand (TRAIL)-mediated apoptosis $(280,281)$. Likewise, CK2 inhibition sensitizes HeLa cells to ionizing radiation (282). Cells within the tumor microenvironment similarly rely on CK2 activity in response to various signals emanating from the tumor cells. CK2 inhibition reduced endothelial cell migration and chemotaxis disrupting neoangiogenesis and hematopoetic stem cell recruitment (229). These studies indicate that CK2 inhibition has limited utility as a single 
agent but may play an important role as a key component in combination therapies where diminished CK2 activity abolishes a drug resistant phenotype.

To date, only two CK2 inhibitors have entered clinical trials: Silimitasertib and CIGB-300 (NCT01199718, NCT00891280, NCT02128282, NCT01639625, NCT01639638). Silmitasertib is a benzonaphthyridine derivative that binds CK2 $\alpha$ with high specificity that inhibits catalytic activity at low concentrations (201). Crystal structure and computational modeling confirm that Silmitasertib-bound CK2 $\alpha$ exhibits the open conformation in the Hinge/ $\alpha \mathrm{D}$ region and a stretched glycine-rich p-loop, confirming the ATP competitive nature of this inhibitor (201). Silmitasertib treatment in murine glioma and breast cancer models blunts NF-KB, PI3K/AKT, and JAK/STAT3 signaling. This results in diminished cell cycle progression, cell viability, and cell migration $(246,283)$. Inhibitor studies in lung and prostate cancer models demonstrate significantly reduced tumor cell proliferation $(246,284,285)$. Silmitasertib treated HNSCC cell lines and xenografts display decreased proliferation, increased cell death and loss of stem-like characteristics $(227,246,255)$. Silmitasertib resistance correlates with upregulated MEK-Erk1/2 signaling which can be abrogated using PD-3025901, a selective MEK inhibitor (246).

CIGB-300 is a small peptide inhibitor that blocks CK2 phosphorylation sites at the CK2 substrate phospho-acceptor site $(265,286)$. CIGB-300 administration induces apoptosis in human cell lines as well as in xenograft models $(265,271)$. Phase I studies have found that intralesional CIGB-300 administration is well tolerated and resulted in cervical cancer patient tumor reduction, with some patients experiencing full histological regression $(265,286,287)$. Similar to previous CK2 inhibitor studies, CIGB-300 synergistically enhanced paclitaxel and cisplatin treatment in human lung and cervical cancer xenografts (288). While only a limited sample, these trials demonstrate promising advances in patient treatment and an opportunity to overcome drug resistance to potentially enhance the clinical standard of care for cancer patients. 


\section{Patient-Derived Xenografts (PDXs)}

Modeling human cancer and developing pharmaceutical interventions has largely been carried out using cell lines in 2D culture or subcutaneous and orthotopic xenograft models with minimal efficacy, with only 5-13.4\% of promising preclinical trial drugs achieving success at the clinical level (289-292). Cell line xenografts fail to recapitulate the tumor microenvironment, therein limiting applicability to the human disease (32). In addition, in vitro cell culture methods select for highly proliferative, pro-survival subclonal populations within the tumor, resulting in an artificially increased cellular homogeneity than what is found in patient tumors (292).

Genetically engineered mouse models (GEMMs) allow for modeling tumorigenesis concurrent with disease progression, evaluation of tumor-stroma interactions, and immune surveillance of the tumor. These traits collectively allow for greater biological relevancy in these models than cellbased systems (293-295). GEMMs enable cancer researchers to induce tissue specific alterations and temporally regulate specific factors to pinpoint critical stages in tumor onset and progression that cannot be achieved using cell line xenografts $(293,294)$. GEMM studies have a variety of applications including validating oncogenic drivers, determining therapeutic responses, and evaluating drug-resistance and disease recurrence in a manner that closely resembles the human disease $(293,295-298)$. However, GEMMs tend to be time consuming to generate, display less tumor heterogeneity due to reliance on specific oncogenic drivers rather than chromosomal instability and de novo DNA damage, and often fail to predict drug efficacy in heavily pretreated patients (297,299-301). While GEMMs are better able to phenocopy the human disease, certain genetic abnormalities found in human patients cannot be recapitulated in any mouse model due to differences between species $(296,301)$. This is particularly true when observing large chromosomal amplification events common in human patients which have no genetic equivalent in rodents. 
As an alternative to these systems, recent advances in generating immune-compromised mouse strains have allowed for the ability of directly implant intact patient tumor tissue that can survive in mice. These patient-derived xenografts (PDX) models maintain a greater degree of tumor heterogeneity and recapitulate the majority of the genetic landscape found in the patient tumor, thus providing a platform for precision oncology testing (300-307). Directly culturing tumors in vivo maintains many conditions of the tumor microenvironment encountered in the patient, including reduced oxygen levels, nutrient and hormone delivery, and tumor-stromal interactions $(32,293,305)$.

PDX development begins with direct patient tumor sample implantation into immunodeficient mice which maintains many histological and genetic characteristics of the primary patient tumor (302304,306-308). Patient samples for PDX work are typically obtained as either fine needle aspirates (FNAs) or following surgical resection (305). Tumor samples are implanted in the recipient mice as small tumor pieces or as a single-cell suspensions mixed with artificial ECM and growth factors $(299,303-307,309-311)$. Single-cell suspension is achieved by digesting patient stroma with ECM-cleaving proteases and/or mechanical mincing of the tissue $(299,305,310-312)$. These suspension techniques expose tumor cells to harsh chemical and mechanical challenges that reduce cell viability, leading to decreased tumor engraftment and heterogeneity in the mouse (299). PDXs can be implanted or injected into heterotopic (typically subcutaneous flank or subretinal space) or orthotopic sites $(305,309,313)$. Site selection is influenced by several factors including tissue size, the orthotopic organ (both size and accessibility), and the goal of that individual PDX model (tissue expansion vs. modeling tumor phenomenon vs. drug treatment evaluation) $(305,312)$.

Engraftment success depends on a host of factors including inherent tumor aggressiveness, histological grade, tumor composition, sample size, and preservation of endogenous tumor signaling such as resistance to anoikis and apoptosis $(299,302,308,310,313)$. Other extraneous 
factors can also impact engraftment including time (from surgical removal from the patient to implantation in the mouse), tissue temperature, lack of continuous nutrient exposure, and implantation location $(299,311)$.

Nomenclature for PDX development and expansion varies between research groups but is typically denoted using either F0, G0, or P0 for the initial implantation of patient tissue into the mouse $(299,305,313)$. Subsequent implantations following the P0 tumor growth add to the number to indicate how many rounds of growth has occurred within the animal model (ex. P1, P2, P3). A PDX is widely considered to be established and reliable upon reaching P3, assuming that the tumor has not deviated from the characteristics of the primary patient sample $(299,305,313)$. At this point, PDX models are usually utilized for drug evaluations or tumor progression studies rather than tissue expansion/PDX establishment that occurs during the first three passages $(299,305,313)$. Criteria for passaging the PDX tumor into the subsequent mouse are highly variable due to differing Institutional Animal Care and Use Committee (IACUC) standards and tumor monitoring techniques. Passaging is commonly performed when the tumor reaches $\sim 1 \mathrm{~cm}$ in the largest dimension. At this point, tumor tissue is removed, processed then reimplanted (313). During tissue processing the tumor mass is broken down into small pieces, several of which may be evaluated for tumor drift or utilized for deriving novel primary cell lines (305).

A critical step in establishing PDX tumors is avoiding host rejection of the tumor xenograft. This is largely achieved using immunodeficient mouse models for tissue implantation (Table 1). Immune compromised mice were first discovered by N.R. Grist in 1962, which were later characterized by Dr. S.P. Flanagan as mice lacking functional T cells (314). In 1983 Bosma et al. published the first severe combined immunodeficient (SCID) mouse model, showing deficiency in functional B and T cell populations (315). Non-obese diabetic (NOD) mice developed to study diabetes mellitus were soon discovered to contain several cellular immunological deficiencies, including dysfunctional dendritic, macrophage, and NK cells (316). Crossing these two stains 
generated the first NOD/SCID mice capable of enhanced human tumor engraftment rates compare to either prior line (317). Combining the NOD/SCID phenotype with IL2RY ${ }^{\text {null }}$ (NSG) mice further enhanced human tumor tissue engraftment and reduced incidence of spontaneous lymphomas that frequently occur from implanted human tumors in the immune-permissive environment (318). While additional immunocompromised mouse stains have been established, none have achieved the widespread utilization of the stains described above $(299,319)$. NSG mice are currently the most commonly utilized model due to their combined adaptive and innate immune response impairment resulting in improved tumor engraftment $(299,305,313,318,320)$. Tumor implantation in NSG mice shows also allows for prolonged survival of associated patient tumor infiltrating lymphocytes (TILs) up to 9 weeks post implantation, allowing for short-term study of patient immune response in this system $(305,321)$. In order to further improve human tumor engraftment, transgenic mice expressing human stem cell factor (SCF), granulocyte-macrophage - colony stimulating factor (GM-CSF), and interleukin (IL)-3 in the NSG background have been established and are termed NSG-SGM3 mice (322). These specific murine cytokines show little cross-reactivity with their human ortholog receptors and have been attributed to poor engraftment of human hematopoetic stem cells and leukemia xenografts, which is countered by the expression of these human cytokines (322).

\begin{tabular}{l|l|l|l} 
Mouse Strain & Modifications & Advantages & \multicolumn{1}{c}{ Disadvantages } \\
\hline Nude & $\begin{array}{l}\text { No functional T } \\
\text { cells }\end{array}$ & $\begin{array}{l}\text { Allows for human tumor } \\
\text { engraftment, hairless } \\
\text { phenotype allows for easier } \\
\text { subcutaneous tumor } \\
\text { monitoring, radioresistant }\end{array}$ & $\begin{array}{l}\text { Lowest human tumor } \\
\text { engraftment rate, } \\
\text { functional B and NK } \\
\text { cells, T cell function } \\
\text { improves with age, } \\
\text { intact complement }\end{array}$ \\
\hline SCID & $\begin{array}{l}\text { No functional B } \\
\text { and T cells }\end{array}$ & $\begin{array}{l}\text { Higher allogeneic and } \\
\text { xenogeneic engraftment rate } \\
\text { than nudes (above), lacks } \\
\text { complement }\end{array}$ & $\begin{array}{l}\text { Functional NK cells, } \\
\text { spontaneous } \\
\text { lymphoma } \\
\text { development }\end{array}$ \\
\hline NODISCID & $\begin{array}{l}\text { No functional B } \\
\text { and T cells; } \\
\text { dendritic, } \\
\text { macrophage, }\end{array}$ & $\begin{array}{l}\text { Low dendritic, macrophage, } \\
\text { and NK cell activity, lacks } \\
\text { complement, engrafts } \\
\text { hematopoietic cancer cell lines }\end{array}$ & $\begin{array}{l}\text { Radiosensitive, higher } \\
\text { rate of spontaneous } \\
\text { lymphoma } \\
\text { development }\end{array}$ \\
\hline
\end{tabular}




\begin{tabular}{|c|c|c|c|}
\hline & $\begin{array}{l}\text { and NK cell } \\
\text { impairment }\end{array}$ & & \\
\hline Rag2y $y^{\text {null }}$ & $\begin{array}{l}\text { No functional B, } \\
T \text {, and NK cells }\end{array}$ & $\begin{array}{l}\text { High human tumor } \\
\text { engraftment rate, low rate of } \\
\text { spontaneous lymphoma } \\
\text { development, radioresistant }\end{array}$ & $\begin{array}{l}\text { Functional dendritic } \\
\text { cells and } \\
\text { macrophages, intact } \\
\text { complement }\end{array}$ \\
\hline NSG & $\begin{array}{l}\text { No functional B, } \\
\text { NK, and T cells; } \\
\text { dendritic cell } \\
\text { and } \\
\text { macrophage } \\
\text { impairment }\end{array}$ & $\begin{array}{l}\text { Higher allogeneic and } \\
\text { xenogeneic engraftment rate } \\
\text { than all stains listed above, } \\
\text { low rate of spontaneous } \\
\text { lymphoma development, lacks } \\
\text { complement }\end{array}$ & $\begin{array}{l}\text { Poor lymph node } \\
\text { development, } \\
\text { maintains tumor } \\
\text { stromal cells for a short } \\
\text { duration ( }-9 \text { weeks), } \\
\text { radiosensitive }\end{array}$ \\
\hline NSG-SGM3 & $\begin{array}{l}\text { No functional B, } \\
\text { NK, and T cells; } \\
\text { dendritic cell } \\
\text { and } \\
\text { macrophage } \\
\text { impairment } \\
\text { Human SCF, } \\
\text { GM-CSF, and } \\
\text { IL-3 expression }\end{array}$ & $\begin{array}{l}\text { Highest allogeneic and } \\
\text { xenogeneic engraftment rate, } \\
\text { low rate of spontaneous } \\
\text { lymphoma, maintains cytokine } \\
\text { sensitive tumor } \\
\text { subpopulations, lacks } \\
\text { complement }\end{array}$ & $\begin{array}{l}\text { Poorly characterized, } \\
\text { slightly deficient lymph } \\
\text { node development, } \\
\text { radiosensitive }\end{array}$ \\
\hline
\end{tabular}

\section{Table 1. Immunocompromised mouse models}

While these advances have increased human tumor engraftment in mice, they require the loss of host immune surveillance. This makes such models unsuitable for studying tumor-immune interactions. To reestablish proper immune system interactions without compromising engraftment rates, current development in the field is centered on producing mice with a humanized immune system. In these models, human CD34 ${ }^{+}$hematopoietic stem cells (HSCs) are selected from bone marrow, cytokine-mobilized peripheral blood, or umbilical cord blood samples. Cells are injected into an immunodeficient recipient mouse's circulation to seed the marrow and function as in the patient $(318,323,324)$. Humanized mice express a wide array of immune lineage cell subtypes and are fully capable of eliciting the expected immune response when challenged $(318,323)$. Not only do these models provide paracrine signaling factors lost in a standard PDX model, they also provide tumor immune surveillance and can be utilized in cancer immunotherapy preclinical studies. 
PDX models have been successfully established for many tumor types (299). Several HNSCC PDX models exist, covering both HPV+ and HPV- subtypes (311,312,325-328). As seen in other tumor PDX models, engraftment rates vary based on the tissue of origin (312). Surprisingly, neither recurrence nor HPV status show any effect on PDX establishment, but these PDXs recapitulate overall survival differences seen in such patients $(311,312)$. The only factor that appears to influence PDX engraftment is metastatic development in the patient prior to surgical resection, but this is inconsistent across cohorts (311,325-327). Epigenetic marker analysis shows that while tumor cell lines are significantly different from primary tumors, established PDXs maintain identical methylation signatures to the primary tumor from which they are derived (329). Preclinical studies demonstrate enrichment for resistance to cisplatin and susceptibility to diaziquone in HNSCC PDXs, which correlates with p53 mutation and cyclin D1 amplification in specific tumor samples $(328,330)$. These data recapitulate findings from various HNSCC clinical trials $(331,332)$. One study showed PI3K enrichment in Cetuximab-resistant HNSCC tumors which respond to PX-866, a PI3K inhibitor, treatment demonstrating predictive therapeutic intervention based on genomic and proteomic markers (312).

Several studies have evaluated specific tumor subpopulations, seeking to identify the HNSCC CSCs which are display enhanced tumor initiating capacity and drug resistance $(307,310,333)$. Establishing treatment modalities to target these distinct subpopulations is key in developing second line therapies and preventing disease recurrence.

PDX models exhibit responses similar to the patient when exposed to clinical therapeutics $(302,334,335)$. The successful translation of PDX treatment to clinical efficacy prompted the National Cancer Institute $(\mathrm{NCl})$ to shift its drug screening program from an established panel of cell lines to a PDX cohort (336). Likewise, the Novartis Institutes for BioMedical Research published a large drug screening study utilizing a library of 1,075 PDX tumors (337). PDX samples can be evaluated post-treatment to identify shifts in tumor expression that may provide 
additional insight towards secondary treatments $(312,338)$. For instance, identifying an expanding drug-resistant subpopulation susceptible to another chemotherapeutic agent that was previously undetectable in the untreated sample provides a beneficial change in clinical intervention $(312,334,338,339)$. One study found that a drug-resistant tumor was addicted to the drug itself, which regressed following treatment cessation (334). This same study also demonstrated that periodic treatment, rather than continual dosing, delayed progress to drug-resistant disease and prevented drug-reliant subpopulation development (334). Several studies have run co-clinical trials where the patient and matched PDX are treated with identical therapeutics, with PDX sample analysis allowing clearer observation of patient tumor alterations that can potentially shift clinical intervention for that individual (340-343). This represents an initial foray into personalized or precision medicine where the genomic and proteomic tumor characteristics govern patient care, rather than conventional therapy where clinicians rely upon gross tumor characteristics including tumor size, grade, and stage to determine patient treatment $(339,344,345)$.

While PDXs represent an advance in characterizing and in preclinical testing of human tumors, there are several limitations to this model. Not every tumor sample engrafts in the animal to form a tumor. Some tumors that do engraft grow too slowly to allow time for clinicians to practically adjust patient care. Additionally, mouse stromal cells quickly replace their human stromal counterparts within the tumor and do not always mimic behaviors found in patient tumor tissue. Similarly, alterations in drug metabolism, targeting, and tumor vascularity can confound PDX drug responses. On the practical side, one must account for the increased cost associated with immunodeficient mouse cohorts along with the time commitment to implant tumors, monitor progress, and passage tissue into subsequent mice. Newly developed models such as the NSGSGM3 can alleviate some biological deficiencies but come at increased expense and/or shortened therapeutic window. Despite these short comings, PDX preclinical and co-clinical trials enhance 
translational robustness to patient care and pave the way towards enhanced precision medicine care in oncology. 


\section{References}

1. Rothenberg SM, Ellisen LW. The molecular pathogenesis of head and neck squamous cell carcinoma. J Clin Invest. 2012;122:1951-7.

2. Leemans $\mathrm{CR}$, Braakhuis BJM, Brakenhoff $\mathrm{RH}$. The molecular biology of head and neck cancer. Nat Rev Cancer. 2011;11:9-22.

3. Amin MB, Edge SB, American Joint Committee on Cancer. AJCC cancer staging manual.

4. Masterson L, Moualed D, Liu ZW, Howard JEF, Dwivedi RC, Tysome JR, et al. Deescalation treatment protocols for human papillomavirus-associated oropharyngeal squamous cell carcinoma: A systematic review and meta-analysis of current clinical trials. Eur J Cancer. 2014;50:2636-48.

5. Chaturvedi AK, Engels EA, Pfeiffer RM, Hernandez BY, Xiao W, Kim E, et al. Human papillomavirus and rising oropharyngeal cancer incidence in the United States. J Clin Oncol. 2011;29:4294-301.

6. Benson E, Li R, Eisele D, Fakhry C. The clinical impact of HPV tumor status upon head and neck squamous cell carcinomas. Oral Oncol. 2014;50:565-74.

7. Rautava J, Syrjänen S. Biology of human papillomavirus infections in head and neck carcinogenesis. Head Neck Pathol. 2012;6 Suppl 1:S3-15.

8. Rampias T, Sasaki C, Psyrri A. Molecular mechanisms of HPV induced carcinogenesis in head and neck. Oral Oncol. 2014;50:356-63.

9. Keck MK, Zuo Z, Khattri A, Stricker TP, Brown CD, Imanguli M, et al. Integrative Analysis of Head and Neck Cancer Identifies Two Biologically Distinct HPV and Three Non-HPV Subtypes. Clin Cancer Res. 2015;21:870-81.

10. Seiwert TY, Zuo Z, Keck MK, Khattri A, Pedamallu CS, Stricker T, et al. Integrative and comparative genomic analysis of HPV-positive and HPV-negative head and neck squamous cell carcimonas. Clin cancer Res. 2014;

11. Zhang Y, Koneva LA, Virani S, Arthur AE, Virani A, Hall PB, et al. Subtypes of HPVPositive Head and Neck Cancers Are Associated with HPV Characteristics, Copy Number Alterations, PIK3CA Mutation, and Pathway Signatures. Clin Cancer Res. 2016;22:4735-45.

12. O'Rorke MA, Ellison M V, Murray LJ, Moran M, James J, Anderson LA. Human papillomavirus related head and neck cancer survival: a systematic review and metaanalysis. Oral Oncol. 2012;48:1191-201.

13. Rivera C, Venegas B. Histological and molecular aspects of oral squamous cell carcinoma. Oncol Lett. 2014;8:7-11.

14. Takes RP, Rinaldo A, Silver CE, Haigentz M, Woolgar JA, Triantafyllou A, et al. Distant metastases from head and neck squamous cell carcinoma. Part I. Basic aspects. Oral Oncol. 2012;48:775-9.

15. López F, Rodrigo JP, Silver CE, Haigentz M, Bishop JA, Strojan P, et al. Cervical lymph node metastases from remote primary tumor sites. Eisele D, editor. Head Neck. 2016;38:E2374-85.

16. Markwell S, Weed S. Tumor and Stromal-Based Contributions to Head and Neck 
Squamous Cell Carcinoma Invasion. Cancers (Basel). 2015;7:382-406.

17. Friedl P, Locker J, Sahai E, Segall JE. Classifying collective cancer cell invasion. Nat Cell Biol. 2012;14:777-83.

18. Friedl $\mathrm{P}$, Alexander S. Cancer invasion and the microenvironment: plasticity and reciprocity. Cell. Elsevier Inc.; 2011;147:992-1009.

19. Sanz-Moreno V, Gadea G, Ahn J, Paterson H, Marra P, Pinner S, et al. Rac activation and inactivation control plasticity of tumor cell movement. Cell. 2008;135:510-23.

20. Sahai E, Marshall CJ. Differing modes of tumour cell invasion have distinct requirements for Rho/ROCK signalling and extracellular proteolysis. Nat Cell Biol. 2003;5:711-9.

21. Parri M, Chiarugi P. Rac and Rho GTPases in cancer cell motility control. Cell Commun Signal. 2010;8:23.

22. Crespo P, Calvo F, Sanz-Moreno V. Ras and Rho GTPases on the move. Bioarchitecture. 2011;1:200-4.

23. Ridley AJ. Rho GTPase signalling in cell migration. Curr Opin Cell Biol. 2015;36:103-12.

24. Jansen S, Gosens R, Wieland T, Schmidt M. Paving the Rho in cancer metastasis: Rho GTPases and beyond. Pharmacol Ther. 2018;183:1-21.

25. Chan AY, Raft S, Bailly M, Wyckoff JB, Segall JE, Condeelis JS. EGF stimulates an increase in actin nucleation and filament number at the leading edge of the lamellipod in mammary adenocarcinoma cells. J Cell Sci. 1998;111 ( Pt 2):199-211.

26. Chhabra ES, Higgs HN. The many faces of actin: matching assembly factors with cellular structures. Nat Cell Biol. 2007;9:1110-21.

27. Friedl $P$, Wolf K. Proteolytic interstitial cell migration: a five-step process. Cancer Metastasis Rev. 2009;28:129-35.

28. Bravo-Cordero JJ, Hodgson L, Condeelis J. Directed cell invasion and migration during metastasis. Curr Opin Cell Biol. 2012;24:277-83.

29. Eddy RJ, Weidmann MD, Sharma VP, Condeelis JS. Tumor Cell Invadopodia: Invasive Protrusions that Orchestrate Metastasis. Trends Cell Biol. Elsevier Ltd; 2017;27:595-607.

30. Gaggioli C, Hooper S, Hidalgo-Carcedo C, Grosse R, Marshall JF, Harrington K, et al. Fibroblast-led collective invasion of carcinoma cells with differing roles for RhoGTPases in leading and following cells. Nat Cell Biol. 2007;9:1392-400.

31. Scott RW, Hooper S, Crighton D, Li A, König I, Munro J, et al. LIM kinases are required for invasive path generation by tumor and tumor-associated stromal cells. J Cell Biol. 2010;191:169-85.

32. De Wever O, Mareel M. Role of tissue stroma in cancer cell invasion. J Pathol. 2003;200:429-47.

33. Dumortier JG, Martin S, Meyer D, Rosa FM, David NB. Collective mesendoderm migration relies on an intrinsic directionality signal transmitted through cell contacts. Proc Natl Acad Sci U S A. 2012;109:16945-50.

34. Yamaguchi $\mathrm{H}$, Condeelis $\mathrm{J}$. Regulation of the actin cytoskeleton in cancer migration and 
invasion. Biochem Biophys Acta. 2007;1773:642-52.

35. Rottner K, Faix J, Bogdan S, Linder S, Kerkhoff E. Actin assembly mechanisms at a glance. J Cell Sci. 2017;130:3427-35.

36. Siton-Mendelson O, Bernheim-Groswasser A. Functional Actin Networks under Construction: The Cooperative Action of Actin Nucleation and Elongation Factors. Trends Biochem Sci. 2017;42:414-30.

37. Pollard TD, Borisy GG. Cellular motility driven by assembly and disassembly of actin filaments. Cell Feb 21, 2003 page 453-65.

38. Campellone KG, Welch MD. A nucleator arms race: cellular control of actin assembly. Nat Rev Mol Cell Biol. 2010;11:237-51.

39. Gligorijevic B, Wyckoff J, Yamaguchi H, Wang Y, Roussos ET, Condeelis J. N-WASPmediated invadopodium formation is involved in intravasation and lung metastasis of mammary tumors. J Cell Sci. 2012;125:724-34.

40. Quintavalle M, Elia L, Condorelli G, Courtneidge SA. MicroRNA control of podosome formation in vascular smooth muscle cells in vivo and in vitro. J Cell Biol. 2010;189:1322.

41. Aung A, Seo YN, Lu S, Wang Y, Jamora C, del Álamo JC, et al. 3D Traction Stresses Activate Protease-Dependent Invasion of Cancer Cells. Biophys J. 2014;107:2528-37.

42. Di Martino J, Henriet E, Ezzoukhry Z, Mondal C, Bravo-Cordero JJ, Moreau V, et al. 2D and 3D Matrices to Study Linear Invadosome Formation and Activity. J Vis Exp. 2017;

43. Di Martino J, Henriet E, Ezzoukhry Z, Goetz JG, Moreau V, Saltel F. The microenvironment controls invadosome plasticity. J Cell Sci. 2016;

44. Petrie RJ, Gavara N, Chadwick RS, Yamada KM. Nonpolarized signaling reveals two distinct modes of 3D cell migration. J Cell Biol. 2012;197:439-55.

45. Murphy DA, Courtneidge SA. The "ins" and "outs" of podosomes and invadopodia: characteristics, formation and function. Nat Rev Mol Cell Biol. Nature Publishing Group; 2011;12:413-26.

46. Gligorijevic B, Bergman A, Condeelis J. Multiparametric classification links tumor microenvironments with tumor cell phenotype. PLoS Biol. Public Library of Science; 2014;12:e1001995.

47. Seano G, Chiaverina G, Gagliardi PA, di Blasio L, Puliafito A, Bouvard C, et al. Endothelial podosome rosettes regulate vascular branching in tumour angiogenesis. Nat Cell Biol. 2014;16:931-41.

48. Chen WT, Chen JM, Parsons SJ, Parsons JT. Local degradation of fibronectin at sites of expression of the transforming gene product pp60src. Nature. 1985;316:156-8.

49. Ayala I, Baldassarre M, Giacchetti G, Caldieri G, Tete S, Luini A, et al. Multiple regulatory inputs converge on cortactin to control invadopodia biogenesis and extracellular matrix degradation. J Cell Sci. 2008;121:369-78.

50. Clark ES, Whigham AS, Yarbrough WG, Weaver AM. Cortactin is an essential regulator of matrix metalloproteinase secretion and extracellular matrix degradation in invadopodia. Cancer Res. 2007;67:4227-35. 
51. Nakahara H, Howard L, Thompson EW, Sato H, Seiki M, Yeh Y, et al.

Transmembrane/cytoplasmic domain-mediated membrane type 1-matrix metalloprotease docking to invadopodia is required for cell invasion. Proc Natl Acad Sci U S A. 1997;94:7959-64.

52. Schoumacher M, Goldman RD, Louvard D, Vignjevic DM. Actin, microtubules, and vimentin intermediate filaments cooperate for elongation of invadopodia. J Cell Biol. 2010;189:541-56.

53. Beaty BT, Condeelis J. Digging a little deeper: The stages of invadopodium formation and maturation. Eur J Cell Biol. NIH Public Access; 2014;93:438-44.

54. Yamaguchi H, Lorenz M, Kempiak S, Sarmieto C, Coniglio S, Symons M, et al. Molecular mechanisms of invadopodium formation: the role of the N-WASP-Arp2/3 complex pathway and cofilin. J Cell Biol. 2005;168:441-52.

55. Mader CC, Oser M, Magalhaes MAO, Bravo-Cordero JJ, Condeelis J, Koleske AJ, et al. An EGFR-Src-Arg-Cortactin pathway mediates functional maturation of invadopodia and breast cancer cell invasion. Cancer Res. 2011;71:1730-41.

56. Bowden ET, Barth M, Thomas D, Glazer RI, Mueller SC. An invasion-related complex of cortactin, paxillin and PKCmu associates with invadopodia at sites of extracellular matrix degradation. Oncogene. 1999;18:4440-9.

57. Artym V V, Zhang Y, Seillier-Moiseiwitsch F, Yamada KM, Mueller SC. Dynamic interactions of cortactin and membrane type 1 matrix metalloproteinase at invadopodia: defining the stages of invadopodia formation and function. Cancer Res. 2006;66:303443.

58. Sharma VP, Eddy R, Entenberg D, Kai M, Gertler FB, Condeelis J. Tks5 and SHIP2 regulate invadopodium maturation, but not initiation, in breast carcinoma cells. Curr Biol. 2013;23:2079-89.

59. Courtneidge SA, Azucena EF, Pass I, Seals DF, Tesfay L. The Src Substrate Tks5, Podosomes (Invadopodia), and Cancer Cell Invasion. Cold Spring Harb Symp Quant Biol. 2005;70:167-71.

60. Seals DF, Azucena EFJ, Pass I, Tesfay L, Gordon R, Woodrow M, et al. The adaptor protein Tks5/Fish is required for podosome formation and function, and for the proteasedriven invasion of cancer cells. Cancer Cell. 2005;7:155-65.

61. Beaty BT, Sharma VP, Bravo-Cordero JJ, Simpson MA, Eddy RJ, Koleske AJ, et al. Beta1 integrin regulates Arg to promote invadopodial maturation and matrix degradation. Mol Biol Cell. 2013;24:1661-75.

62. Arber S, Barbayannis FA, Hanser H, Schneider C, Stanyon CA, Bernard O, et al. Regulation of actin dynamics through phosphorylation of cofilin by LIM-kinase. Nature. 1998;393:805-9.

63. Blanchoin L, Pollard TD, Mullins RD. Interactions of ADF/cofilin, Arp2/3 complex, capping protein and profilin in remodeling of branched actin filament networks. Curr Biol. 2000;10:1273-82.

64. Oser M, Condeelis J. The cofilin activity cycle in lamellipodia and invadopodia. J Cell Biochem. 2009;108:1252-62. 
65. Oser M, Mader CC, Gil-Henn H, Magalhaes M, Bravo-Cordero JJ, Koleske AJ, et al. Specific tyrosine phosphorylation sites on cortactin regulate Nck1-dependent actin polymerization in invadopodia. J Cell Sci. 2010;123:3662-73.

66. Lapetina S, Mader CC, Machida K, Mayer BJ, Koleske AJ. Arg interacts with cortactin to promote adhesion-dependent cell edge protrusion. J Cell Biol. 2009;185:503-19.

67. Stylli SS, Stacey TTI, Verhagen AM, Xu SS, Pass I, Courtneidge SA, et al. Nck adaptor proteins link Tks5 to invadopodia actin regulation and ECM degradation. J Cell Sci. 2009;122:2727-40.

68. Crimaldi L, Courtneidge SA, Gimona M. Tks5 recruits AFAP-110, p190RhoGAP, and cortactin for podosome formation. Exp Cell Res. 2009;315:2581-92.

69. Linder S, Nelson D, Weiss M, Aepfelbacher M. Wiskott-Aldrich syndrome protein regulates podosomes in primary human macrophages. Proc Natl Acad Sci U S A. 1999;96:9648-53.

70. Oser M, Yamaguchi H, Mader CC, Bravo-Cordero JJ, Arias M, Chen X, et al. Cortactin regulates cofilin and $\mathrm{N}$-WASp activities to control the stages of invadopodium assembly and maturation. J Cell Biol. 2009;186:571-87.

71. MacGrath SM, Koleske AJ. Cortactin in cell migration and cancer at a glance. J Cell Sci. Company of Biologists; 2012;125:1621-6.

72. Schnoor M, Stradal TE, Rottner K. Cortactin: Cell Functions of A Multifaceted ActinBinding Protein. Trends Cell Biol. 2018;28:79-98.

73. Lizarraga F, Poincloux R, Romao M, Montagnac G, Le Dez G, Bonne I, et al. Diaphanous-Related Formins Are Required for Invadopodia Formation and Invasion of Breast Tumor Cells. Cancer Res. 2009;69:2792-800.

74. Steffen A, Le Dez G, Poincloux R, Recchi C, Nassoy P, Rottner K, et al. MT1-MMPdependent invasion is regulated by TI-VAMP/VAMP7. Curr Biol. 2008;18:926-31.

75. Jacob A, Prekeris R. The regulation of MMP targeting to invadopodia during cancer metastasis. Front Cell Dev Biol. 2015;3:4.

76. Bravo-Cordero JJ, Marrero-Diaz R, Megías D, Genís L, García-Grande A, García MA, et al. MT1-MMP proinvasive activity is regulated by a novel Rab8-dependent exocytic pathway. EMBO J. 2007;26:1499-510.

77. Gimona M, Buccione R, Courtneidge SA, Linder S. Assembly and biological role of podosomes and invadopodia. Curr. Opin. Cell Biol. 2008. page 235-41.

78. Van Waes C. Cell adhesion and regulatory molecules involved in tumor formation, hemostasis, and wound healing. Head Neck. 1995;17:140-7.

79. Philippar U, Roussos ET, Oser M, Yamaguchi H, Kim H-D, Giampieri S, et al. A Mena invasion isoform potentiates EGF-induced carcinoma cell invasion and metastasis. Dev Cell. 2008;15:813-28.

80. Li A, Dawson JC, Forero-Vargas M, Spence HJ, Yu X, König I, et al. The actin-bundling protein fascin stabilizes actin in invadopodia and potentiates protrusive invasion. Curr Biol. 2010;20:339-45.

81. Kelly T, Mueller SC, Yeh Y, Chen WT. Invadopodia promote proteolysis of a wide variety 
of extracellular matrix proteins. J Cell Physiol. 1994;158:299-308.

82. Mueller SC, Yeh Y, Chen WT. Tyrosine phosphorylation of membrane proteins mediates cellular invasion by transformed cells. J Cell Biol. 1992;119:1309-25.

83. Alexander NR, Branch KM, Parekh A, Clark ES, Iwueke IC, Guelcher SA, et al. Extracellular matrix rigidity promotes invadopodia activity. Curr Biol. 2008;18:1295-9.

84. Ghersi G, Dong H, Goldstein LA, Yeh Y, Hakkinen L, Larjava HS, et al. Regulation of Fibroblast Migration on Collagenous Matrix by a Cell Surface Peptidase Complex. J Biol Chem. 2002;277:29231-41.

85. Redondo-Muñoz J, Escobar-Díaz E, Samaniego R, Terol MJ, García-Marco JA, GarcíaPardo A. MMP-9 in B-cell chronic lymphocytic leukemia is up-regulated by alpha4beta1 integrin or CXCR4 engagement via distinct signaling pathways, localizes to podosomes, and is involved in cell invasion and migration. Blood. 2006;108:3143-51.

86. Monsky WL, Lin CY, Aoyama A, Kelly T, Akiyama SK, Mueller SC, et al. A potential marker protease of invasiveness, seprase, is localized on invadopodia of human malignant melanoma cells. Cancer Res. 1994;54:5702-10.

87. Wang Z, Zhang F, He J, Wu P, Tay LWR, Cai M, et al. Binding of PLD2-Generated Phosphatidic Acid to KIF5B Promotes MT1-MMP Surface Trafficking and Lung Metastasis of Mouse Breast Cancer Cells. Dev Cell. 2017;43:186-197.e7.

88. Kumar S, Das A, Barai A, Sen S. MMP Secretion Rate and Inter-invadopodia Spacing Collectively Govern Cancer Invasiveness. Biophys J. 2018;114:650-62.

89. El Azzouzi K, Wiesner C, Linder S. Metalloproteinase MT1-MMP islets act as memory devices for podosome reemergence. J Cell Biol. 2016;213:109-25.

90. Morris DC, Popp JL, Tang LK, Gibbs HC, Schmitt E, Chaki SP, et al. Nck deficiency is associated with delayed breast carcinoma progression and reduced metastasis. Mol Biol Cell. 2017;28:3500-16.

91. Yu X, Zech T, McDonald L, Gonzalez EG, Li A, Macpherson I, et al. N-WASP coordinates the delivery and F-actin-mediated capture of MT1-MMP at invasive pseudopods. J Cell Biol. 2012;199:527-44.

92. Castro-Castro A, Marchesin V, Monteiro P, Lodillinsky C, Rossé C, Chavrier P. Cellular and Molecular Mechanisms of MT1-MMP-Dependent Cancer Cell Invasion. Annu Rev Cell Dev Biol. 2016;32:555-76.

93. Caldieri G, Giacchetti G, Beznoussenko G, Attanasio F, Ayala I, Buccione R. Invadopodia biogenesis is regulated by caveolin-mediated modulation of membrane cholesterol levels. J Cell Mol Med. 2009;13:1728-40.

94. Yamaguchi H, Takeo Y, Yoshida S, Kouchi Z, Nakamura Y, Fukami K. Lipid rafts and caveolin-1 are required for invadopodia formation and extracellular matrix degradation by human breast cancer cells. Cancer Res. 2009;69:8594-602.

95. Sakurai-Yageta M, Recchi C, Le Dez G, Sibarita J-B, Daviet L, Camonis J, et al. The interaction of IQGAP1 with the exocyst complex is required for tumor cell invasion downstream of Cdc42 and RhoA. J Cell Biol. 2008;181:985-98.

96. Linklater E, Jewett CE, Prekeris R. Polarized Membrane Trafficking in Development and 
Disease: From Epithelia Polarization to Cancer Cell Invasion. In: Houston DW, editor. Cell polarity Dev Dis. 2018. page 121-39.

97. Hebbrecht T, Van Audenhove I, Zwaenepoel O, Verhelle A, Gettemans J. VCA nanobodies target N-WASp to reduce invadopodium formation and functioning. Komarova Y, editor. PLoS One. 2017;12:e0185076.

98. Bertier L, Hebbrecht T, Mettepenningen E, De Wit N, Zwaenepoel O, Verhelle A, et al. Nanobodies targeting cortactin proline rich, helical and actin binding regions downregulate invadopodium formation and matrix degradation in SCC-61 cancer cells. Biomed Pharmacother. 2018;102:230-41.

99. Magalhaes MAO, Larson DR, Mader CC, Bravo-Cordero JJ, Gil-Henn H, Oser M, et al. Cortactin phosphorylation regulates cell invasion through a $\mathrm{pH}$-dependent pathway. J Cell Biol. 2011;195:903-20.

100. Juin a., Billottet C, Moreau V, Destaing O, Albiges-Rizo C, Rosenbaum J, et al. Physiological type I collagen organization induces the formation of a novel class of linear invadosomes. Mol Biol Cell. 2012;23:297-309.

101. Albiges-Rizo C, Destaing O, Fourcade B, Planus E, Block MR. Actin machinery and mechanosensitivity in invadopodia, podosomes and focal adhesions. J Cell Sci. 2009;122:3037-49.

102. Destaing O, Block MR, Planus E, Albiges-Rizo C. Invadosome regulation by adhesion signaling. Curr Opin Cell Biol. 2011;23:597-606.

103. Linder S, Wiesner C. Feel the force: Podosomes in mechanosensing. Exp Cell Res. 2016;343:67-72.

104. Wiesner C, Le-Cabec V, El Azzouzi K, Maridonneau-Parini I, Linder S. Podosomes in space: macrophage migration and matrix degradation in $2 \mathrm{D}$ and $3 \mathrm{D}$ settings. Cell Adh Migr. 2014;8:179-91.

105. You W-K, Stallcup W. Localization of VEGF to Vascular ECM Is an Important Aspect of Tumor Angiogenesis. Cancers (Basel). 2017;9:97.

106. Harper K, R. Lavoie R, Charbonneau M, Brochu-Gaudreau K, Dubois CM. The Hypoxic Tumor Microenvironment Promotes Invadopodia Formation and Metastasis through LPA1 Receptor and EGFR Cooperation. Mol Cancer Res. 2018;16:1601-13.

107. Hoffmann C, Mao X, Brown-Clay J, Moreau F, Al Absi A, Wurzer H, et al. Hypoxia promotes breast cancer cell invasion through HIF-1a-mediated up-regulation of the invadopodial actin bundling protein CSRP2. Sci Rep. 2018;8:10191.

108. Wu H, Parsons JT. Cortactin, an 80/85-Kilodalton pp60Src substrate, is a Filamentous Actin-binding Protein Enriched in the Cell Cortex. J Cell Biol. 1993;120:1417-26.

109. Wu H, Reynolds a B, Kanner SB, Vines RR, Parsons JT. Identification and characterization of a novel cytoskeleton-associated pp60src substrate. Mol Cell Biol. 1991;11:5113-24.

110. Yin M, Ma W, An L. Cortactin in cancer cell migration and invasion. Oncotarget. Impact Journals, LLC; 2017;8:88232-43.

111. Dedes KJ, Lopez-Garcia M-A, Geyer FC, Lambros MBK, Savage K, Vatcheva R, et al. 
Cortactin gene amplification and expression in breast cancer: a chromogenic in situ hybridisation and immunohistochemical study. Breast Cancer Res Treat. 2010;124:65366.

112. Li Y, Tondravi M, Liu J, Smith E, Haudenschild CC, Kaczmarek M, et al. Cortactin Potentiates Bone Metastasis of Breast Cancer Cells. Cancer Res. 2001;61:6906-11.

113. Yuan B-Z, Zhou X, Zimonjic DB, Durkin ME, Popescu NC. Amplification and Overexpression of the EMS 1 Oncogene, a Possible Prognostic Marker, in Human Hepatocellular Carcinoma. J Mol Diagnostics. 2003;5:48-53.

114. Hirakawa $\mathrm{H}$, Shibata $\mathrm{K}$, Nakayama $\mathrm{T}$. Localization of cortactin is associated with colorectal cancer development. Int J Oncol. 2009;35:1271-6.

115. Schuuring E, Verhoeven E, Litvinov S, Michalides RJAM. The product of the EMS1 gene, amplified and overexpressed in human carcinomas, is homologous to a v-src substrate and is located in cell-substratum contact sites. Mol Cell Biol. 1993;13:2891-8.

116. Luo M-L, Shen X-M, Zhang Y, Wei F, Xu X, Cai Y, et al. Amplification and overexpression of CTTN (EMS1) contribute to the metastasis of esophageal squamous cell carcinoma by promoting cell migration and anoikis resistance. Cancer Res. 2006;66:11690-9.

117. Gao H-W, Yu C-P, Lee H-S, Nieh S, Chiang C-P, Wang W-M, et al. Fascin, cortactin and survivin expression of melanocytic neoplasms and association with clinicopathological parameters and anatomic locations in Chinese people. Eur J Dermatol. 2010;20:293301.

118. Ambrosio EP, Rosa FE, Domingues MAC, Villacis RAR, Coudry R de A, Tagliarini JV, et al. Cortactin is associated with perineural invasion in the deep invasive front area of laryngeal carcinomas. Hum Pathol. 2011;42:1221-9.

119. Clark ES, Brown B, Whigham AS, Kochaishvili a, Yarbrough WG, Weaver a M. Aggressiveness of HNSCC tumors depends on expression levels of cortactin, a gene in the 11q13 amplicon. Oncogene. 2009;28:431-44.

120. Gibcus JH, Mastik MF, Menkema L, de Bock GH, Kluin PM, Schuuring E, et al. Cortactin expression predicts poor survival in laryngeal carcinoma. Br J Cancer. 2008;98:950-5.

121. Hofman P, Butori C, Havet K, Hofman V, Selva E, Guevara N, et al. Prognostic significance of cortactin levels in head and neck squamous cell carcinoma: comparison with epidermal growth factor receptor status. Br J Cancer. 2008;98:956-64.

122. Alavi S, Namazie A, Calcaterra TC, Wang MB, Srivatsan ES. Clinical application of fluorescence in situ hybridization for chromosome 11q13 analysis in head and neck cancer. Laryngoscope. 1999;109:874-9.

123. Jin C, Jin Y, Gisselsson D, Wennerberg J, Wah TS, Strömbäck B, et al. Molecular cytogenetic characterization of the 11q13 amplicon in head and neck squamous cell carcinoma. Cytogenet Genome Res. 2006;115:99-106.

124. Meredith SD, Levine PA, Burns JA, Gaffey MJ, Boyd JC, Weiss LM, et al. Chromosome $11 q 13$ amplification in head and neck squamous cell carcinoma. Association with poor prognosis. Arch Otolaryngol Head Neck Surg. 1995;121:790-4.

125. Rodrigo JP, Garcı LA, Ramos S, Lazo PS, Sua C, García LA, et al. EMS1 Gene Amplification Correlates with Poor Prognosis in Squamous Cell Carcinomas of the Head 
and Neck. Clin cancer Res. 2000;6:3177-82.

126. Schuuring E, Verhoeven E, Mooi WJ, Michalides RJAM. Identification and cloning of two overexpressed genes, U21B31/PRAD1 and EMS1, within the amplified chromosome $11 q 13$ region in human carcinomas. Oncogene. 1992;7:355-61.

127. Kwek SS, Roy R, Zhou H, Climent J, Martinez-Climent JA, Fridlyand J, et al. Co-amplified genes at $8 p 12$ and $11 q 13$ in breast tumors cooperate with two major pathways in oncogenesis. Oncogene. 2009;28:1892-903.

128. Ammer AG, Weed SA. Cortactin branches out: roles in regulating protrusive actin dynamics. Cell Motil Cytoskeleton. 2008;65:687-707.

129. Sugahara K, Michikawa Y, Ishikawa K, Shoji Y, Iwakawa M, Shibahara T, et al. Combination effects of distinct cores in 11q13 amplification region on cervical lymph node metastasis of oral squamous cell carcinoma. Int J Oncol. 2011;39:761-9.

130. Hui R, Ball JR, Macmillan RD, Kenny FS, Prall OW, Campbell DH, et al. EMS1 gene expression in primary breast cancer: relationship to cyclin D1 and oestrogen receptor expression and patient survival. Oncogene. 1998;17:1053-9.

131. Chuma M, Sakamoto M, Yasuda J, Fujii G, Nakanishi K, Tsuchiya A, et al. Overexpression of cortactin is involved in motility and metastasis of hepatocellular carcinoma. J Hepatol. 2004;41:629-36.

132. Rothschild BL, Shim AH, Ammer AG, Kelley LC, Irby KB, Head J a, et al. Cortactin overexpression regulates actin-related protein $2 / 3$ complex activity, motility, and invasion in carcinomas with chromosome 11q13 amplification. Cancer Res. 2006;66:8017-25.

133. Cowieson NP, King G, Cookson D, Ross I, Huber T, Hume DA, et al. Cortactin adopts a globular conformation and bundles actin into sheets. J Biol Chem. 2008;283:16187-93.

134. Kirkbride KC, Sung BH, Sinha S, Weaver AM. Cortactin: a multifunctional regulator of cellular invasiveness. Cell Adh Migr. 2011;5:187-98.

135. Weed S a, Parsons JT. Cortactin: coupling membrane dynamics to cortical actin assembly. Oncogene. 2001;20:6418-34.

136. Blanchoin L, Amann KJ, Higgs HN, Marchand J, Kaiser DA, Pollard TD. Direct observation of dendritic actin filament networks nucleated by Arp2/3 complex and WASP / Scar proteins. Nature. 2000;404:1007-11.

137. Weaver AM, Heuser JE, Karginov A V., Lee W, Parsons JT, Cooper JA. Interaction of cortactin and N-WASp with Arp2/3 complex. Curr Biol. 2002;12:1270-8.

138. Weed S a, Du Y, Parsons JT. Translocation of cortactin to the cell periphery is mediated by the small GTPase Rac1. J Cell Sci. 1998;111 ( Pt 1:2433-43.

139. Weed SA, Karginov A V., Schafer DA, Weaver AM, Kinley AW, Cooper JA, et al. Cortactin localization to sites of actin assembly in lamellipodia requires interactions with F-actin and the Arp2/3 complex. J Cell Biol. 2000;151:29-40.

140. Pollard TD. Regulation of Actin Filament Assembly by Arp2/3 Complex and Formins. Annu Rev Biophys Biomol Struct. Annual Reviews; 2007;36:451-77.

141. Miglarese MR, Mannion-Henderson J, Wu H, Parsons JT, Bender TP. The protein tyrosine kinase substrate cortactin is differentially expressed in murine $\mathrm{B}$ lymphoid 
tumors. Oncogene. 1994;9:1989-97.

142. Du Y, Weed SA, Xiong WC, Marshall TD, Parsons JT. Identification of a novel cortactin $\mathrm{SH} 3$ domain-binding protein and its localization to growth cones of cultured neurons. Mol Cell Biol. 1998;18:5838-51.

143. Daly RJ. Cortactin signalling and dynamic actin networks. Biochem J. 2004;382:13-25.

144. Helgeson LA, Prendergast JG, Wagner AR, Rodnick-Smith M, Nolen BJ. Interactions with Actin Monomers, Actin Filaments and Arp2/3 Complex Define the Roles of WASP Family Proteins and Cortactin in Coordinately Regulating Branched Actin Networks. J Biol Chem. 2014;289:1-26.

145. Helgeson $L$ a, Nolen BJ. Mechanism of synergistic activation of Arp2/3 complex by cortactin and N-WASP. Elife. 2013;2:e00884.

146. Zhang X, Yuan Z, Zhang Y, Yong S, Salas-Burgos A, Koomen J, et al. HDAC6 modulates cell motility by altering the acetylation level of cortactin. Mol Cell. 2007;27:197-213.

147. Zhang Y, Zhang M, Dong H, Yong S, Li X, Olashaw N, et al. Deacetylation of cortactin by SIRT1 promotes cell migration. Oncogene. 2009;28:445-60.

148. Luxton GWG, Gundersen GG. HDAC6-pack: cortactin acetylation joins the brew. Dev Cell. 2007;13:161-2.

149. Weaver AM, Karginov A V., Kinley AW, Weed SA, Li Y, Parsons JT, et al. Cortactin promotes and stabilizes Arp2/3-induced actin filament network formation. Curr Biol. 2001;11:370-4.

150. Huang C, Tandon NN, Greco NJ, Ni Y, Wang T, Zhan X. Proteolysis of platelet cortactin by calpain. J Biol Chem. 1997;272:19248-52.

151. Perrin BJ, Amann KJ, Huttenlocher A. Proteolysis of cortactin by calpain regulates membrane protrusion during cell migration. Mol Biol Cell. 2006;17:239-50.

152. Webb BA, Zhou S, Eves R, Shen L, Jia L, Mak AS. Phosphorylation of cortactin by p21activated kinase. Arch Biochem Biophys. 2006;456:183-93.

153. Campbell DH, Sutherland RL, Daly RJ. Signaling Pathways and Structural Domains Required for Phosphorylation of EMS1 / Cortactin Signaling. Cancer Res. 1999;59:537685.

154. Evans J V, Ammer AG, Jett JE, Bolcato C a, Breaux JC, Martin KH, et al. Src binds cortactin through an SH2 domain cystine-mediated linkage. J Cell Sci. 2012;125:618597.

155. McNiven MA, Kim L, Krueger EW, Orth JD, Cao H, Wong TW. Regulated interactions between dynamin and the actin-binding protein cortactin modulate cell shape. J Cell Biol. 2000;151:187-98.

156. Tomar A, Lawson C, Ghassemian M, Schlaepfer DD. Cortactin as a target for FAK in the regulation of focal adhesion dynamics. Hotchin NA, editor. PLoS One. 2012;7:e44041.

157. Martin KH, Jeffery ED, Grigera PR, Shabanowitz J, Hunt DF, Parsons JT. Cortactin phosphorylation sites mapped by mass spectrometry. J Cell Sci. 2006;119:2851-3.

158. Tehrani S, Tomasevic N, Weed S, Sakowicz R, Cooper J a. Src phosphorylation of 
cortactin enhances actin assembly. Proc Natl Acad Sci U S A. 2007;104:11933-8.

159. Boyle SN, Michaud GA, Schweitzer B, Predki PF, Koleske AJ. A critical role for cortactin phosphorylation by Abl-family kinases in PDGF-induced dorsal-wave formation. Curr Biol. 2007;17:445-51.

160. Bougnères L, Girardin SE, Weed SA, Karginov A V, Olivo-Marin J-C, Parsons JT, et al. Cortactin and Crk cooperate to trigger actin polymerization during Shigella invasion of epithelial cells. J Cell Biol. 2004;166:225-35.

161. Mizutani K, Miki H, He H, Maruta H, Takenawa T. Essential role of neural Wiskot-Aldrich syndrome protein in podosome formation and degradation of extracellular matrix in srctransformed fibroblasts. Cancer Res. 2002;62:669-74.

162. Stuible M, Dubé N, Tremblay ML. PTP1B regulates cortactin tyrosine phosphorylation by targeting Tyr446. J Biol Chem. 2008;283:15740-6.

163. Weidmann MD, Surve CR, Eddy RJ, Chen X, Gertler FB, Sharma VP, et al. MenalNV dysregulates cortactin phosphorylation to promote invadopodium maturation. Sci Rep. 2016;6:36142.

164. Ammer AG, Kelley LC, Hayes KE, Evans J V, Lopez-skinner A, Martin KH, et al. Saracatinib impairs head and neck squamous cell carcinoma invasion by disruption invadopodia function. J Cancer Sci Ther. 2009;1:52-61.

165. Nicholas NS, Pipili A, Lesjak MS, Wells CM. Differential role for PAK1 and PAK4 during the invadopodia lifecycle. Small GTPases. 2017;1-7.

166. Martinez-quiles N, Ho HH, Kirschner MW, Ramesh N, Geha RS. Erk / Src Phosphorylation of Cortactin Acts as a Switch On-Switch Off Mechanism That Controls Its Ability To Activate N-WASP. Mol Cell Biol. 2004;24:5269-80.

167. Kelley LC, Hayes KE, Ammer AG, Martin KH, Weed SA. Revisiting the ERK/Src cortactin switch. Commun Integr Biol. 2011;4:205-7.

168. Kelley LC, Hayes KE, Ammer AG, Martin KH, Weed SA. Cortactin phosphorylated by ERK1/2 localizes to sites of dynamic actin regulation and is required for carcinoma lamellipodia persistence. PLoS One. 2010;5:e13847.

169. De Kimpe L, Janssens K, Derua R, Armacki M, Goicoechea S, Otey C, et al. Characterization of cortactin as an in vivo protein kinase $D$ substrate: interdependence of sites and potentiation by Src. Cell Signal. 2009;21:253-63.

170. Sroka R, Van Lint J, Katz S-F, Schneider MR, Kleger A, Paschke S, et al. Cortactin is a scaffolding platform for the $\mathrm{E}$-cadherin adhesion complex and is regulated by protein kinase D1 phosphorylation. J Cell Sci. 2016;129:2416-29.

171. Eiseler T, Hausser A, De Kimpe L, Van Lint J, Pfizenmaier K. Protein kinase D controls actin polymerization and cell motility through phosphorylation of cortactin. J Biol Chem. 2010;285:18672-83.

172. Eiseler T, Schmid MA, Topbas F, Pfizenmaier K, Hausser A. PKD is recruited to sites of actin remodelling at the leading edge and negatively regulates cell migration. FEBS Lett. 2007;581:4279-87.

173. Ren G, Crampton MS, Yap AS. Cortactin: Coordinating adhesion and the actin 
cytoskeleton at cellular protrusions. Cell Motil Cytoskeleton. 2009;66:865-73.

174. Galletta BJ, Chuang DY, Cooper JA. Distinct roles for Arp2/3 regulators in actin assembly and endocytosis. Kirschner MW, editor. PLoS Biol. 2008;6:e1.

175. Xu X-P, Rouiller I, Slaughter BD, Egile C, Kim E, Unruh JR, et al. Three-dimensional reconstructions of Arp2/3 complex with bound nucleation promoting factors. EMBO J. Nature Publishing Group; 2012;31:236-47.

176. Uruno T, Liu J, Li Y, Smith N, Zhan X. Sequential interaction of actin-related proteins 2 and 3 (Arp2/3) complex with neural Wiscott-Aldrich syndrome protein (N-WASP) and cortactin during branched actin filament network formation. J Biol Chem.

2003;278:26086-93.

177. Jeannot P, Nowosad A, Perchey RT, Callot C, Bennana E, Katsube T, et al. p27Kip1 promotes invadopodia turnover and invasion through the regulation of the PAK1/Cortactin pathway. Elife. 2017;6.

178. Moshfegh Y, Bravo-Cordero JJ, Miskolci V, Condeelis J, Hodgson L. A Trio-Rac1-Pak1 signalling axis drives invadopodia disassembly. Nat Cell Biol. Nature Publishing Group; 2014;16:571-83.

179. Jeannot P, Nowosad A, Perchey RT, Callot C, Bennana E, Katsube T, et al. p27 Kip1 promotes invadopodia turnover and invasion through the regulation of the PAK1/Cortactin pathway. Elife. 2017;6.

180. Goicoechea SM, Zinn A, Awadia SS, Snyder K, Garcia-Mata R. A RhoG-mediated signaling pathway that modulates invadopodia dynamics in breast cancer cells. J Cell Sci. 2017;130:jcs.195552.

181. Badowski C, Pawlak G, Grichine A, Chabadel A, Oddou C, Jurdic P, et al. Paxillin Phosphorylation Controls Invadopodia/Podosomes Spatiotemporal Organization. Ginsberg M, editor. Mol Biol Cell. 2008;19:633-45.

182. Calle Y, Carragher NO, Thrasher AJ, Jones GE. Inhibition of calpain stabilises podosomes and impairs dendritic cell motility. J Cell Sci. 2006;119:2375-85.

183. Hoskin V, Szeto A, Ghaffari A, Greer PA, Côté GP, Elliott BE. Ezrin regulates focal adhesion and invadopodia dynamics by altering calpain activity to promote breast cancer cell invasion. Mol Biol Cell. 2015;26:3464-79.

184. Urrutia R, Henley JR, Cook T, McNiven MA. The dynamins: redundant or distinct functions for an expanding family of related GTPases? Proc Natl Acad Sci U S A. 1997;94:377-84.

185. Schafer DA, Weed SA, Binns D, Karginov A V, Parsons JT, Cooper JA. Dynamin2 and cortactin regulate actin assembly and filament organization. Curr Biol. 2002;12:1852-7.

186. Mooren OL, Kotova TI, Moore AJ, Schafer DA. Dynamin2 GTPase and cortactin remodel actin filaments. J Biol Chem. 2009;284:23995-4005.

187. Cao H, Orth JD, Chen J, Weller SG, Heuser JE, McNiven MA. Cortactin is a component of clathrin-coated pits and participates in receptor-mediated endocytosis. Mol Cell Biol. 2003;23:2162-70.

188. Kaksonen M, Peng HB, Rauvala H. Association of cortactin with dynamic actin in 
lamellipodia and on endosomal vesicles. J Cell Sci. 2000;113 Pt 24:4421-6.

189. Clark ES, Weaver AM. A new role for cortactin in invadopodia: regulation of protease secretion. Eur J Cell Biol. 2008;87:581-90.

190. Zhao G, Zhang H, Huang Z, Lv L, Yan F. Cortactin and Exo70 mediated invasion of hepatoma carcinoma cells by MMP-9 secretion. Mol Biol Rep. 2016;43:407-14.

191. Chua MMJ, Ortega CE, Sheikh A, Lee M, Abdul-Rassoul H, Hartshorn KL, et al. CK2 in Cancer: Cellular and Biochemical Mechanisms and Potential Therapeutic Target. Pharmaceuticals (Basel). Multidisciplinary Digital Publishing Institute (MDPI); 2017;10.

192. Bidwai AP, Hanna DE, Glover C V. Purification and characterization of casein kinase II (CKII) from delta cka1 delta cka2 Saccharomyces cerevisiae rescued by Drosophila CKII subunits. The free catalytic subunit of casein kinase II is not toxic in vivo. J Biol Chem. 1992;267:18790-6.

193. Meggio F, Boldyreff B, Marin O, Marchiori F, Perich JW, Issinger OG, et al. The effect of polylysine on casein-kinase-2 activity is influenced by both the structure of the protein/peptide substrates and the subunit composition of the enzyme. Eur J Biochem. 1992;205:939-45.

194. Franchin C, Borgo C, Cesaro L, Zaramella S, Vilardell J, Salvi M, et al. Re-evaluation of protein kinase CK2 pleiotropy: new insights provided by a phosphoproteomics analysis of CK2 knockout cells. Cell Mol Life Sci. Springer International Publishing; 2017;1-16.

195. Trembley JH, Chen Z, Unger G, Slaton J, Kren BT, Van Waes C, et al. Emergence of protein kinase CK2 as a key target in cancer therapy. Biofactors. NIH Public Access; 2010;36:187-95.

196. Faust M, Montenarh M. Subcellular localization of protein kinase CK2. A key to its function? Cell Tissue Res. 2000;301:329-40.

197. Krek W, Maridor G, Nigg EA. Casein kinase II is a predominantly nuclear enzyme. J Cell Biol. 1992;116:43-55.

198. Meggio F, Pinna $L$ a. One-thousand-and-one substrates of protein kinase CK2? FASEB J. 2003;17:349-68.

199. Grankowski N, Boldyreff B, Issinger OG. Isolation and characterization of recombinant human casein kinase II subunits alpha and beta from bacteria. Eur J Biochem. 1991;198:25-30.

200. Wei T, Tao M. Human erythrocyte casein kinase II: characterization and phosphorylation of membrane cytoskeletal proteins. Arch Biochem Biophys. 1993;307:206-16.

201. Srivastava A, Hirota T, Irle S, Tama F. Conformational dynamics of human protein kinase CK2 $\alpha$ and its effect on function and inhibition. Proteins Struct Funct Bioinforma. 2018;86:344-53.

202. Niefind K, Raaf J, Issinger O-G. Protein kinase CK2 in health and disease: Protein kinase CK2: from structures to insights. Cell Mol Life Sci. 2009;66:1800-16.

203. Di Maira G, Brustolon F, Bertacchini J, Tosoni K, Marmiroli S, Pinna LA, et al. Pharmacological inhibition of protein kinase CK2 reverts the multidrug resistance phenotype of a CEM cell line characterized by high CK2 level. Oncogene. 2007;26:6915- 
26.

204. Tawfic S, Yu S, Wang H, Faust R, Davis A, Ahmed K. Protein kinase CK2 signal in neoplasia. Histol Histopathol. 2001;16:573-82.

205. Ahmed K, Yenice S, Davis A, Goueli SA. Association of casein kinase 2 with nuclear chromatin in relation to androgenic regulation of rat prostate. Proc Natl Acad Sci U S A. 1993;90:4426-30.

206. Martel V, Filhol O, Nueda A, Cochet C. Dynamic localization/association of protein kinase CK2 subunits in living cells: a role in its cellular regulation? Ann N Y Acad Sci. 2002;973:272-7.

207. Wang H, Yu S, Davis AT, Ahmed K. Cell cycle dependent regulation of protein kinase CK2 signaling to the nuclear matrix. J Cell Biochem. 2003;88:812-22.

208. Yu S, Davis AT, Guo C, Green JE, Ahmed K. Differential targeting of protein kinase CK2 to the nuclear matrix upon transient overexpression of its subunits. J Cell Biochem. 1999;74:127-34.

209. Salvi M, Sarno S, Marin O, Meggio F, Itarte E, Pinna LA. Discrimination between the activity of protein kinase CK2 holoenzyme and its catalytic subunits. FEBS Lett. 2006;580:3948-52.

210. Guo C, Yu S, Davis AT, Wang H, Green JE, Ahmed K. A potential role of nuclear matrixassociated protein kinase CK2 in protection against drug-induced apoptosis in cancer cells. J Biol Chem. 2001;276:5992-9.

211. Hériché JK, Chambaz EM. Protein kinase CK2alpha is a target for the Abl and Bcr-Abl tyrosine kinases. Oncogene. 1998;17:13-8.

212. Wang H, Davis A, Yu S, Ahmed K. Response of cancer cells to molecular interruption of the CK2 signal. Mol Cell Biochem. 2001;227:167-74.

213. Slaton JW, Unger GM, Sloper DT, Davis AT, Ahmed K. Induction of apoptosis by antisense CK2 in human prostate cancer xenograft model. Mol Cancer Res. 2004;2:71221.

214. Yu S, Wang H, Davis A, Ahmed K. Consequences of CK2 signaling to the nuclear matrix. Mol Cell Biochem. 2001;227:67-71.

215. Ahmed K. Nuclear matrix and protein kinase CK2 signaling. Crit Rev Eukaryot Gene Expr. 1999;9:329-36.

216. Ji H, Wang J, Nika H, Hawke D, Keezer S, Ge Q, et al. EGF-induced ERK activation promotes CK2-mediated disassociation of alpha-Catenin from beta-Catenin and transactivation of beta-Catenin. Mol Cell. 2009;36:547-59.

217. Orlandini M, Semplici F, Ferruzzi R, Meggio F, Pinna LA, Oliviero S. Protein kinase CK2alpha' is induced by serum as a delayed early gene and cooperates with Ha-ras in fibroblast transformation. J Biol Chem. 1998;273:21291-7.

218. Di Maira G, Salvi M, Arrigoni G, Marin O, Sarno S, Brustolon F, et al. Protein kinase CK2 phosphorylates and upregulates Akt/PKB. Cell Death Differ. 2005;12:668-77.

219. Jia H, Liu Y, Xia R, Tong C, Yue T, Jiang J, et al. Casein kinase 2 promotes Hedgehog signaling by regulating both smoothened and Cubitus interruptus. J Biol Chem. 
2010;285:37218-26.

220. Zheng Y, Qin H, Frank SJ, Deng L, Litchfield DW, Tefferi A, et al. A CK2-dependent mechanism for activation of the JAK-STAT signaling pathway. Blood. 2011;118:156-66.

221. O'Brien KA, Lemke SJ, Cocke KS, Rao RN, Beckmann RP. Casein kinase 2 binds to and phosphorylates BRCA1. Biochem Biophys Res Commun. 1999;260:658-64.

222. Kapoor M, Lozano G. Functional activation of $p 53$ via phosphorylation following DNA damage by UV but not gamma radiation. Proc Natl Acad Sci U S A. 1998;95:2834-7.

223. Keller DM, Lu H. p53 serine 392 phosphorylation increases after UV through induction of the assembly of the CK2.hSPT16.SSRP1 complex. J Biol Chem. 2002;277:50206-13.

224. Li Y, Keller DM, Scott JD, Lu H. CK2 phosphorylates SSRP1 and inhibits its DNA-binding activity. J Biol Chem. NIH Public Access; 2005;280:11869-75.

225. Scaglioni PP, Yung TM, Cai LF, Erdjument-Bromage $H$, Kaufman AJ, Singh B, et al. A CK2-Dependent Mechanism for Degradation of the PML Tumor Suppressor. Cell. 2006;126:269-83.

226. Torres $\mathrm{J}$, Pulido R. The tumor suppressor PTEN is phosphorylated by the protein kinase CK2 at its $C$ terminus. Implications for PTEN stability to proteasome-mediated degradation. J Biol Chem. 2001;276:993-8.

227. Brown MS, Diallo OT, Hu M, Ehsanian R, Yang X, Arun P, et al. CK2 Modulation of NF- B, TP53, and the Malignant Phenotype in Head and Neck Cancer by Anti-CK2 Oligonucleotides In vitro or In vivo via Sub-50-nm Nanocapsules. Clin Cancer Res. 2010;16:2295-307.

228. Kramerov AA, Saghizadeh M, Pan H, Kabosova A, Montenarh M, Ahmed K, et al. Expression of protein kinase CK2 in astroglial cells of normal and neovascularized retina. Am J Pathol. 2006;168:1722-36.

229. Kramerov AA, Saghizadeh M, Caballero S, Shaw LC, Li Calzi S, Bretner M, et al. Inhibition of protein kinase CK2 suppresses angiogenesis and hematopoietic stem cell recruitment to retinal neovascularization sites. Mol Cell Biochem. 2008;316:177-86.

230. Sarrouilhe D, Filhol O, Leroy D, Bonello G, Baudry M, Chambaz EM, et al. The tight association of protein kinase CK2 with plasma membranes is mediated by a specific domain of its regulatory beta-subunit. Biochim Biophys Acta. 1998;1403:199-210.

231. Ruzzene M, Brunati AM, Sarno S, Marin O, Donella-Deana A, Pinna LA. Ser/Thr phosphorylation of hematopoietic specific protein 1 (HS1): implication of protein kinase CK2. Eur J Biochem. 2000;267:3065-72.

232. Mendoza MC. Phosphoregulation of the WAVE regulatory complex and signal integration. Semin Cell Dev Biol. Elsevier Ltd; 2013;24:272-9.

233. Pocha SM, Cory GO. WAVE2 is regulated by multiple phosphorylation events within its VCA domain. Cell Motil Cytoskeleton. 2009;66:36-47.

234. Cory GOC, Cramer R, Blanchoin L, Ridley AJ. Phosphorylation of the WASP-VCA domain increases its affinity for the Arp2/3 complex and enhances actin polymerization by WASP. Mol Cell. 2003;11:1229-39.

235. Galovic M, Xu D, Areces LB, van der Kammen R, Innocenti M. Interplay between N- 
WASP and CK2 optimizes clathrin-mediated endocytosis of EGFR. J Cell Sci. 2011;124:2001-12.

236. Ura S, Pollitt AY, Veltman DM, Morrice NA, Machesky LM, Insall RH. Pseudopod growth and evolution during cell movement is controlled through SCAR/WAVE dephosphorylation. Curr Biol. 2012;22:553-61.

237. Markwell SM, Ammer AG, Interval ET, Allen JL, Papenberg RA, Castano JE, et al. Cortactin Phosphorylation by Casein Kinase 2 Regulates Actin-Related Protein 2/3 Complex Activity, Invadopodia Function and Tumor Cell Invasion. Mol cancer Res. 2019;in press.

238. Serrano L, Hernández MA, Díaz-Nido J, Avila J. Association of casein kinase II with microtubules. Exp Cell Res. 1989;181:263-72.

239. Crute BE, Van Buskirk RG. A casein kinase-like kinase phosphorylates beta-tubulin and may be a microtubule-associated protein. J Neurochem. 1992;59:2017-23.

240. Serrano L, Díaz-Nido J, Wandosell F, Avila J. Tubulin phosphorylation by casein kinase II is similar to that found in vivo. J Cell Biol. 1987;105:1731-9.

241. Karki S, Tokito MK, Holzbaur EL. Casein kinase II binds to and phosphorylates cytoplasmic dynein. J Biol Chem. 1997;272:5887-91.

242. Moreno FJ, Avila J. Phosphorylation of stathmin modulates its function as a microtubule depolymerizing factor. Mol Cell Biochem. 1998;183:201-9.

243. Greenwood JA, Scott CW, Spreen RC, Caputo CB, Johnson G V. Casein kinase II preferentially phosphorylates human tau isoforms containing an amino-terminal insert. Identification of threonine 39 as the primary phosphate acceptor. J Biol Chem. 1994;269:4373-80.

244. Faust M, Schuster N, Montenarh M. Specific binding of protein kinase CK2 catalytic subunits to tubulin. FEBS Lett. 1999;462:51-6.

245. Ortega CE, Seidner Y, Dominguez I. Mining CK2 in cancer. PLoS One. 2014;9:e115609.

246. Bian Y, Han J, Kannabiran V, Mohan S, Cheng H, Friedman J, et al. MEK inhibitor PD0325901 overcomes resistance to CK2 inhibitor CX-4945 and exhibits anti-tumor activity in head and neck cancer. Int J Biol Sci. Ivyspring International Publisher; 2015;11:41122.

247. Landesman-Bollag E, Song DH, Romieu-Mourez R, Sussman DJ, Cardiff RD, Sonenshein GE, et al. Protein kinase CK2: signaling and tumorigenesis in the mammary gland. Mol Cell Biochem. 2001;227:153-65.

248. Liu R, Wang X, Chen GY, Dalerba P, Gurney A, Hoey T, et al. The prognostic role of a gene signature from tumorigenic breast-cancer cells. N Engl J Med. 2007;356:217-26.

249. Gapany M, Faust R a, Tawfic S, Davis a, Adams GL, Ahmed K. Association of elevated protein kinase CK2 activity with aggressive behavior of squamous cell carcinoma of the head and neck. Mol Med. 1995;1:659-66.

250. Faust RA, Gapany M, Tristani P, Davis A, Adams GL, Ahmed K. Elevated protein kinase CK2 activity in chromatin of head and neck tumors: association with malignant transformation. Cancer Lett. 1996;101:31-5. 
251. Faust RA, Niehans G, Gapany M, Hoistad D, Knapp D, Cherwitz D, et al. Subcellular immunolocalization of protein kinase CK2 in normal and carcinoma cells. Int J Biochem Cell Biol. 1999;31:941-9.

252. Faust RA, Tawfic S, Davis AT, Bubash LA, Ahmed K. Antisense oligonucleotides against protein kinase CK2-alpha inhibit growth of squamous cell carcinoma of the head and neck in vitro. Head Neck. 2000;22:341-6.

253. Yenice S, Davis AT, Goueli SA, Akdas A, Limas C, Ahmed K. Nuclear casein kinase 2 (CK-2) activity in human normal, benign hyperplastic, and cancerous prostate. Prostate. 1994;24:11-6.

254. Su Y-W, Xie T-X, Sano D, Myers JN. IL-6 stabilizes Twist and enhances tumor cell motility in head and neck cancer cells through activation of casein kinase 2. PLoS One. 2011;6:e19412.

255. Lu H, Yan C, Quan XX, Yang X, Zhang J, Bian Y, et al. CK2 Phosphorylates and Inhibits TAp73 Tumor Suppressor Function to Promote Expression of Cancer Stem Cell Genes and Phenotype in Head and Neck Cancer. Neoplasia. 2014;16:789-800.

256. Landesman-Bollag E, Channavajhala PL, Cardiff RD, Seldin DC. p53 deficiency and misexpression of protein kinase CK2 $\alpha$ collaborate in the development of thymic lymphomas in mice. Oncogene. 1998;16:2965-74.

257. Xu X, Landesman-Bollag E, Channavajhala PL, Seldin DC. Murine protein kinase CK2: gene and oncogene. Mol Cell Biochem. 1999;191:65-74.

258. Kelliher MA, Seldin DC, Leder P. Tal-1 induces T cell acute lymphoblastic leukemia accelerated by casein kinase Ilalpha. EMBO J. 1996;15:5160-6.

259. Channavajhala $P$, Seldin DC. Functional interaction of protein kinase CK2 and c-Myc in lymphomagenesis. Oncogene. 2002;21:5280-8.

260. Zhang S, Long H, Yang Y-L, Wang Y, Hsieh D, Li W, et al. Inhibition of CK2a downregulates Notch1 signalling in lung cancer cells. J Cell Mol Med. 2013;17:854-62.

261. Zhang F, Yang B, Shi S, Jiang X. RNA interference (RNAi) mediated stable knockdown of protein casein kinase 2-alpha (CK2 $\alpha$ ) inhibits migration and invasion and enhances cisplatin-induced apoptosis in HEp-2 laryngeal carcinoma cells. Acta Histochem. 2014;116:1000-6.

262. Duncan JS, Litchfield DW. Too much of a good thing: the role of protein kinase CK2 in tumorigenesis and prospects for therapeutic inhibition of CK2. Biochim Biophys Acta. 2008;1784:33-47.

263. Wang G, Unger G, Ahmad KA, Slaton JW, Ahmed K. Downregulation of CK2 induces apoptosis in cancer cells - A potential approach to cancer therapy. Mol Cell Biochem. 2005;274:77-84.

264. Ahmad KA, Wang G, Slaton J, Unger G, Ahmed K. Targeting CK2 for cancer therapy. Anticancer Drugs. 2005;16:1037-43.

265. Perea SE, Reyes O, Baladron I, Perera Y, Farina H, Gil J, et al. CIGB-300, a novel proapoptotic peptide that impairs the CK2 phosphorylation and exhibits anticancer properties both in vitro and in vivo. Mol Cell Biochem. 2008;316:163-7. 
266. Zandomeni R, Zandomeni MC, Shugar D, Weinmann R. Casein kinase type II is involved in the inhibition by 5,6-dichloro-1-beta-D-ribofuranosylbenzimidazole of specific RNA polymerase II transcription. J Biol Chem. 1986;261:3414-9.

267. Hagiwara M, Inoue S, Tanaka T, Nunoki K, Ito M, Hidaka H. Differential effects of flavonoids as inhibitors of tyrosine protein kinases and serine/threonine protein kinases. Biochem Pharmacol. 1988;37:2987-92.

268. Pagano MA, Meggio F, Ruzzene M, Andrzejewska M, Kazimierczuk Z, Pinna LA. 2Dimethylamino-4,5,6,7-tetrabromo-1H-benzimidazole: a novel powerful and selective inhibitor of protein kinase CK2. Biochem Biophys Res Commun. 2004;321:1040-4.

269. Yim H, Lee YH, Lee $\mathrm{CH}$, Lee SK. Emodin, an anthraquinone derivative isolated from the rhizomes of Rheum palmatum, selectively inhibits the activity of casein kinase II as a competitive inhibitor. Planta Med. 1999;65:9-13.

270. Götz C, Gratz A, Kucklaender U, Jose J. TF — A novel cell-permeable and selective inhibitor of human protein kinase CK2 induces apoptosis in the prostate cancer cell line LNCaP. Biochim Biophys Acta - Gen Subj. 2012;1820:970-7.

271. Benavent Acero F, Capobianco CS, Garona J, Cirigliano SM, Perera Y, Urtreger AJ, et al. CIGB-300, an anti-CK2 peptide, inhibits angiogenesis, tumor cell invasion and metastasis in lung cancer models. Lung Cancer. 2017;107:14-21.

272. Siddiqui-Jain A, Drygin D, Streiner N, Chua P, Pierre F, O'Brien SE, et al. CX-4945, an orally bioavailable selective inhibitor of protein kinase CK2, inhibits prosurvival and angiogenic signaling and exhibits antitumor efficacy. Cancer Res. American Association for Cancer Research; 2010;70:10288-98.

273. Sarno S, Reddy H, Meggio F, Ruzzene M, Davies SP, Donella-Deana A, et al. Selectivity of 4,5,6,7-tetrabromobenzotriazole, an ATP site-directed inhibitor of protein kinase CK2 ('casein kinase-2'). FEBS Lett. 2001;496:44-8.

274. Cozza G, Mazzorana M, Papinutto E, Bain J, Elliott M, di Maira G, et al. Quinalizarin as a potent, selective and cell-permeable inhibitor of protein kinase CK2. Biochem J. 2009;421:387-95.

275. HUNG M-S, XU Z, CHEN Y, SMITH E, MAO J-H, HSIEH D, et al. Hematein, a casein kinase II inhibitor, inhibits lung cancer tumor growth in a murine xenograft model. Int J Oncol. 2013;43:1517-22.

276. Pagano MA, Poletto G, Di Maira G, Cozza G, Ruzzene M, Sarno S, et al. Tetrabromocinnamic Acid (TBCA) and Related Compounds Represent a New Class of Specific Protein Kinase CK2 Inhibitors. ChemBioChem. 2007;8:129-39.

277. Zień P, Bretner M, Zastapiło K, Szyszka R, Shugar D. Selectivity of 4,5,6,7tetrabromobenzimidazole as an ATP-competitive potent inhibitor of protein kinase CK2 from various sources. Biochem Biophys Res Commun. 2003;306:129-33.

278. Sandholt IS, Olsen BB, Guerra B, Issinger O-G. Resorufin: a lead for a new protein kinase CK2 inhibitor. Anticancer Drugs. 2009;20:238-48.

279. Mishra S, Pertz V, Zhang B, Kaur P, Shimada H, Groffen J, et al. Treatment of P190 Bcr/Abl lymphoblastic leukemia cells with inhibitors of the serine/threonine kinase CK2. Leukemia. 2007;21:178-80. 
280. Izeradjene K, Douglas L, Delaney A, Houghton JA. Casein kinase II (CK2) enhances death-inducing signaling complex (DISC) activity in TRAIL-induced apoptosis in human colon carcinoma cell lines. Oncogene. 2005;24:2050-8.

281. Izeradjene K, Douglas L, Delaney A, Houghton JA. Influence of casein kinase II in tumor necrosis factor-related apoptosis-inducing ligand-induced apoptosis in human rhabdomyosarcoma cells. Clin Cancer Res. 2004;10:6650-60.

282. Yamane K, Kinsella TJ. CK2 inhibits apoptosis and changes its cellular localization following ionizing radiation. Cancer Res. 2005;65:4362-7.

283. Gray GKK, McFarland BC, Rowse AL, Gibson SA, Benveniste EN. Therapeutic CK2 inhibition attentuates diverse prosurvival signaling cascades and decreases cell viability in human breast cancer cells. Oncotarget. 2014;5:6484-96.

284. Pierre F, Chua PC, O'Brien SE, Siddiqui-Jain A, Bourbon P, Haddach M, et al. Discovery and SAR of 5-(3-Chlorophenylamino)benzo[ $c$ ][2,6]naphthyridine-8-carboxylic Acid (CX4945), the First Clinical Stage Inhibitor of Protein Kinase CK2 for the Treatment of Cancer. J Med Chem. 2011;54:635-54.

285. Pierre F, Chua PC, O'Brien SE, Siddiqui-Jain A, Bourbon P, Haddach M, et al. Preclinical characterization of CX-4945, a potent and selective small molecule inhibitor of CK2 for the treatment of cancer. Mol Cell Biochem. 2011;356:37-43.

286. Solares AM, Santana A, Baladrón I, Valenzuela C, González CA, Díaz A, et al. Safety and preliminary efficacy data of a novel Casein Kinase 2 (CK2) peptide inhibitor administered intralesionally at four dose levels in patients with cervical malignancies. BMC Cancer. 2009;9:146.

287. Sarduy MR, García I, Coca MA, Perera A, Torres LA, Valenzuela CM, et al. Optimizing CIGB-300 intralesional delivery in locally advanced cervical cancer. $\mathrm{Br} \mathrm{J}$ Cancer. 2015;112:1636-43.

288. PERERA Y, TORO N DEL, GOROVAYA L, FERNANDEZ-DE-COSSIO J, FARINA HG, PEREA SE. Synergistic interactions of the anti-casein kinase 2 CIGB-300 peptide and chemotherapeutic agents in lung and cervical preclinical cancer models. Mol Clin Oncol. 2014;2:935-44.

289. Barretina J, Caponigro G, Stransky N, Venkatesan K, Margolin AA, Kim S, et al. The Cancer Cell Line Encyclopedia enables predictive modelling of anticancer drug sensitivity. Nature. 2012;483:603-7.

290. DiMasi JA, Reichert JM, Feldman L, Malins A. Clinical approval success rates for investigational cancer drugs. Clin Pharmacol Ther. 2013;94:329-35.

291. Hutchinson L, Kirk R. High drug attrition rates-where are we going wrong? Nat Rev Clin Oncol. 2011;8:189-90.

292. Gillet J-P, Calcagno AM, Varma S, Marino M, Green LJ, Vora MI, et al. Redefining the relevance of established cancer cell lines to the study of mechanisms of clinical anticancer drug resistance. Proc Natl Acad Sci U S A. 2011;108:18708-13.

293. Gengenbacher N, Singhal M, Augustin HG. Preclinical mouse solid tumour models: status quo, challenges and perspectives. Nat Rev Cancer. Nature Publishing Group; 2017;17:751-65. 
294. Day C-P, Merlino G, Van Dyke T. Preclinical mouse cancer models: a maze of opportunities and challenges. Cell. NIH Public Access; 2015;163:39-53.

295. Clohessy JG, Pandolfi PP. Mouse hospital and co-clinical trial project-from bench to bedside. Nat Rev Clin Oncol. Nature Publishing Group; 2015;12:491-8.

296. Singh M, Murriel CL, Johnson L. Genetically engineered mouse models: closing the gap between preclinical data and trial outcomes. Cancer Res. American Association for Cancer Research; 2012;72:2695-700.

297. Kersten K, de Visser KE, van Miltenburg MH, Jonkers J. Genetically engineered mouse models in oncology research and cancer medicine. EMBO Mol Med. Wiley-Blackwell; 2017;9:137-53.

298. Singh M, Lima A, Molina R, Hamilton P, Clermont AC, Devasthali V, et al. Assessing therapeutic responses in Kras mutant cancers using genetically engineered mouse models. Nat Biotechnol. Nature Publishing Group; 2010;28:585-93.

299. Jung J, Seol HS, Chang S. The Generation and Application of Patient-Derived Xenograft Model for Cancer Research. Cancer Res Treat. 2018;50:1-10.

300. Alizadeh AA, Aranda V, Bardelli A, Blanpain C, Bock C, Borowski C, et al. Toward understanding and exploiting tumor heterogeneity. Nat Med. 2015;21:846-53.

301. Tabassum DP, Polyak K. Tumorigenesis: It takes a village. Nat Rev Cancer. Nature Publishing Group; 2015;15:473-83.

302. Sivanand S, Peña-Llopis S, Zhao H, Kucejova B, Spence P, Pavia-Jimenez A, et al. A validated tumorgraft model reveals activity of dovitinib against renal cell carcinoma. Sci Transl Med. 2012;4:137ra75.

303. van Weerden WM, de Ridder CM, Verdaasdonk CL, Romijn JC, van der Kwast TH, Schröder $\mathrm{FH}$, et al. Development of seven new human prostate tumor xenograft models and their histopathological characterization. Am J Pathol. 1996;149:1055-62.

304. Reyal F, Guyader C, Decraene C, Lucchesi C, Auger N, Assayag F, et al. Molecular profiling of patient-derived breast cancer xenografts. Breast Cancer Res. 2012;14:R11.

305. Tentler JJ, Tan AC, Weekes CD, Jimeno A, Leong S, Pitts TM, et al. Patient-derived tumour xenografts as models for oncology drug development. Nat Rev Clin Oncol. 2012;9:338-50.

306. Jung J, Lee CH, Seol HS, Choi YS, Kim E, Lee EJ, et al. Generation and molecular characterization of pancreatic cancer patient-derived xenografts reveals their heterologous nature. Oncotarget. 2016;7:62533-46.

307. Basu D, Nguyen T-TK, Montone KT, Zhang G, Wang L-P, Diehl JA, et al. Evidence for mesenchymal-like sub-populations within squamous cell carcinomas possessing chemoresistance and phenotypic plasticity. Oncogene. 2010;29:4170-82.

308. John T, Kohler D, Pintilie M, Yanagawa N, Pham N-A, Li M, et al. The ability to form primary tumor xenografts is predictive of increased risk of disease recurrence in earlystage non-small cell lung cancer. Clin Cancer Res. 2011;17:134-41.

309. Morton CL, Houghton PJ. Establishment of human tumor xenografts in immunodeficient mice. Nat Protoc. 2007;2:247-50. 
310. Prince ME, Sivanandan R, Kaczorowski A, Wolf GT, Kaplan MJ, Dalerba P, et al. Identification of a subpopulation of cells with cancer stem cell properties in head and neck squamous cell carcinoma. Proc Natl Acad Sci. 2007;104:973-8.

311. Kimple RJ, Harari PM, Torres AD, Yang RZ, Soriano BJ, Yu M, et al. Development and characterization of HPV-positive and HPV-negative head and neck squamous cell carcinoma tumorgrafts. Clin Cancer Res. 2013;19:855-64.

312. Keysar SB, Astling DP, Anderson RT, Vogler BW, Bowles DW, Morton JJ, et al. A patient tumor transplant model of squamous cell cancer identifies PI3K inhibitors as candidate therapeutics in defined molecular bins. Mol Oncol. 2013;7:776-90.

313. Jin K, Teng L, Shen Y, He K, Xu Z, Li G. Patient-derived human tumour tissue xenografts in immunodeficient mice: a systematic review. Clin Transl Oncol. 2010;12:473-80.

314. Flanagan SP. "Nude", a new hairless gene with pleiotropic effects in the mouse. Genet Res. 1966;8:295-309.

315. Bosma GC, Custer RP, Bosma MJ. A severe combined immunodeficiency mutation in the mouse. Nature. 1983;301:527-30.

316. Kikutani $\mathrm{H}$, Makino $\mathrm{S}$. The murine autoimmune diabetes model: NOD and related strains. Adv Immunol. 1992;51:285-322.

317. Lapidot T, Fajerman Y, Kollet O. Immune-deficient SCID and NOD/SCID mice models as functional assays for studying normal and malignant human hematopoiesis. J Mol Med (Berl). 1997;75:664-73.

318. Shultz LD, Lyons BL, Burzenski LM, Gott B, Chen X, Chaleff S, et al. Human lymphoid and myeloid cell development in NOD/LtSz-scid IL2R gamma null mice engrafted with mobilized human hemopoietic stem cells. J Immunol. 2005;174:6477-89.

319. Okada S, Vaeteewoottacharn K, Kariya R. Establishment of a Patient-Derived Tumor Xenograft Model and Application for Precision Cancer Medicine. Chem Pharm Bull. 2018;66:225-30.

320. Cao X, Shores EW, Hu-Li J, Anver MR, Kelsall BL, Russell SM, et al. Defective lymphoid development in mice lacking expression of the common cytokine receptor gamma chain. Immunity. 1995;2:223-38.

321. Simpson-Abelson MR, Sonnenberg GF, Takita H, Yokota SJ, Conway TF, Kelleher RJ, et al. Long-term engraftment and expansion of tumor-derived memory T cells following the implantation of non-disrupted pieces of human lung tumor into NOD-scid IL2Rgamma(null) mice. J Immunol. 2008;180:7009-18.

322. Wunderlich M, Chou F-S, Link KA, Mizukawa B, Perry RL, Carroll M, et al. AML xenograft efficiency is significantly improved in NOD/SCID-IL2RG mice constitutively expressing human SCF, GM-CSF and IL-3. Leukemia. 2010;24:1785-8.

323. Wiekmeijer A-S, Pike-Overzet K, Brugman MH, Salvatori DCF, Egeler RM, Bredius RGM, et al. Sustained Engraftment of Cryopreserved Human Bone Marrow CD34(+) Cells in Young Adult NSG Mice. Biores Open Access. 2014;3:110-6.

324. Saito Y, Ellegast JM, Rafiei A, Song Y, Kull D, Heikenwalder M, et al. Peripheral blood CD34+ cells efficiently engraft human cytokine knock-in mice. Blood. American Society of Hematology; 2016;128:1829-33. 
325. Chen J, Milo GE, Shuler CF, Schuller DE. Xenograft growth and histodifferentiation of squamous cell carcinomas of the pharynx and larynx. Oral Surg Oral Med Oral Pathol Oral Radiol Endod. 1996;81:197-202.

326. Zätterström UK, Braakhuis BJ, Wennerberg J, van Dongen GA, Attewell R, Nauta JJ, et al. Growth of xenografted squamous cell carcinoma of the head and neck--possible correlation with patient survival. APMIS. 1992;100:976-80.

327. Wennerberg J, Tropé C, Biörklund A. Heterotransplantation of human head and neck tumours into nude mice. Acta Otolaryngol. 1983;95:183-90.

328. Langdon SP, Hendriks HR, Braakhuis BJ, Pratesi G, Berger DP, Fodstad O, et al. Preclinical phase II studies in human tumor xenografts: a European multicenter follow-up study. Ann Oncol Off J Eur Soc Med Oncol. 1994;5:415-22.

329. Hennessey PT, Ochs MF, Mydlarz WW, Hsueh W, Cope L, Yu W, et al. Promoter methylation in head and neck squamous cell carcinoma cell lines is significantly different than methylation in primary tumors and xenografts. Wang XW, editor. PLoS One. 2011;6:e20584.

330. Henriksson E, Baldetorp B, Borg A, Kjellen E, Akervall J, Wennerberg J, et al. p53 mutation and cyclin D1 amplification correlate with cisplatin sensitivity in xenografted human squamous cell carcinomas from head and neck. Acta Oncol. 2006;45:300-5.

331. Koch WM, Brennan JA, Zahurak M, Goodman SN, Westra WH, Schwab D, et al. p53 mutation and locoregional treatment failure in head and neck squamous cell carcinoma. $\mathrm{J}$ Natl Cancer Inst. 1996;88:1580-6.

332. Cabelguenne A, Blons H, de Waziers I, Carnot F, Houllier AM, Soussi T, et al. p53 alterations predict tumor response to neoadjuvant chemotherapy in head and neck squamous cell carcinoma: a prospective series. J Clin Oncol. 2000;18:1465-73.

333. Basu D, Montone KT, Wang L-P, Gimotty PA, Hammond R, Diehl JA, et al. Detecting and targeting mesenchymal-like subpopulations within squamous cell carcinomas. Cell Cycle. 2011;10:2008-16.

334. Das Thakur M, Salangsang F, Landman AS, Sellers WR, Pryer NK, Levesque MP, et al. Modelling vemurafenib resistance in melanoma reveals a strategy to forestall drug resistance. Nature. 2013;494:251-5.

335. Merk J, Rolff J, Becker M, Leschber G, Fichtner I. Patient-derived xenografts of nonsmall-cell lung cancer: a pre-clinical model to evaluate adjuvant chemotherapy? Eur J Cardiothorac Surg. 2009;36:454-9.

336. Ledford H. US cancer institute to overhaul tumour cell lines. Nature. 2016;530:391-391.

337. Gao H, Korn JM, Ferretti S, Monahan JE, Wang Y, Singh M, et al. High-throughput screening using patient-derived tumor xenografts to predict clinical trial drug response. Nat Med. 2015;21:1318-25.

338. Migliardi G, Sassi F, Torti D, Galimi F, Zanella ER, Buscarino M, et al. Inhibition of MEK and PI3K/mTOR suppresses tumor growth but does not cause tumor regression in patient-derived xenografts of RAS-mutant colorectal carcinomas. Clin Cancer Res. 2012;18:2515-25.

339. Erriquez J, Olivero M, Mittica G, Scalzo MS, Vaira M, De Simone M, et al. Xenopatients 
show the need for precision medicine approach to chemotherapy in ovarian cancer. Oncotarget. 2016;7:26181-91.

340. Garrido-Laguna I, Tan AC, Uson M, Angenendt M, Ma WW, Villaroel MC, et al. Integrated preclinical and clinical development of mTOR inhibitors in pancreatic cancer. Br J Cancer. 2010;103:649-55.

341. Jimeno A, Amador ML, Kulesza P, Wang X, Rubio-Viqueira B, Zhang X, et al. Assessment of celecoxib pharmacodynamics in pancreatic cancer. Mol Cancer Ther. 2006;5:3240-7.

342. Lunardi A, Ala U, Epping MT, Salmena L, Clohessy JG, Webster KA, et al. A co-clinical approach identifies mechanisms and potential therapies for androgen deprivation resistance in prostate cancer. Nat Genet. 2013;45:747-55.

343. Owonikoko TK, Zhang G, Kim HS, Stinson RM, Bechara R, Zhang C, et al. Patientderived xenografts faithfully replicated clinical outcome in a phase II co-clinical trial of arsenic trioxide in relapsed small cell lung cancer. J Transl Med. 2016;14:111.

344. Gonçalves A, Bertucci F, Guille A, Garnier S, Adelaide J, Carbuccia N, et al. Targeted $\mathrm{NGS}$, array-CGH, and patient-derived tumor xenografts for precision medicine in advanced breast cancer: a single-center prospective study. Oncotarget. 2016;7:7942841.

345. Witkiewicz AK, Balaji U, Eslinger C, McMillan E, Conway W, Posner B, et al. Integrated Patient-Derived Models Delineate Individualized Therapeutic Vulnerabilities of Pancreatic Cancer. Cell Rep. 2016;16:2017-31. 


\section{Study 1: Cortactin Phosphorylation by Casein Kinase 2}

\section{Regulates Actin-Related Protein 2/3 Complex Activity,}

\section{Invadopodia Function and Tumor Cell Invasion}

Steven M. Markwell ${ }^{1}$, Amanda G. Ammer ${ }^{1}$, Erik T. Interval ${ }^{2}$, Jessica L. Allen ${ }^{1}$, Brenen W. Papenberg ${ }^{1}$, River A. Hames ${ }^{1}$, Johnathan E. Castaño ${ }^{2}$, Dorothy A. Schafer ${ }^{3}$, Scott A. Weed ${ }^{1 *}$

${ }^{1}$ Program in Cancer Cell Biology, Department of Biochemistry, West Virginia University, Morgantown, WV, ${ }^{2}$ Department of Otolaryngology, Head and Neck Surgery, West Virginia University, Morgantown, WV, ${ }^{3}$ Department of Biology, University of Virginia, Charlottesville, VA.

Running title. CTTN Phosphorylation by CK2 Regulates Invasion

Keywords. Cortactin, HNSCC, Invadopodia, Arp2/3, CK2

Financial support: West Virginia University Cancer Institute and NIH grants P20RR016440, P30RR032138/GM103488, P20GM103434, and P20RR016477.

This work was also supported by the Dorothy D. Radford Endowed Fund, West Virginia University Cancer Institute, the Departments of Neurobiology and Anatomy, and Biochemistry (West Virginia University). This project was additionally supported by the National Institute of General Medical Sciences, U54GM104942. The content is solely the responsibility of the authors and does not necessarily represent the official views of the NIH.

Corresponding Author: "Scott A. Weed, West Virginia University Cancer Institute, P.O. Box 9300, Morgantown, WV 26506, Phone: 304-293-3016, Fax: 304-293-4667, scweed@hsc.wvu.edu.

In Press at Molecular Cancer Research (2019) 


\section{Abstract}

Malregulation of the actin cytoskeleton enhances tumor cell motility and invasion. The actin-binding protein cortactin facilitates branched actin network formation through activation of the actin-related protein (Arp) 2/3 complex. Increased cortactin expression due to gene amplification is observed in head and neck squamous cell carcinoma (HNSCC) and other cancers, corresponding with elevated tumor progression and poor patient outcome. Arp2/3 complex activation is responsible for driving increased migration and extracellular matrix (ECM) degradation by governing invadopodia formation and activity. While cortactin-mediated activation of Arp2/3 complex and invadopodia regulation has been well established, signaling pathways responsible for governing cortactin binding to Arp2/3 are unknown and potentially present a new avenue for anti-invasive therapeutic targeting. Here we identify casein kinase (CK) $2 \alpha$ phosphorylation of cortactin as a negative regulator of Arp2/3 binding. CK2 $\alpha$ directly phosphorylates cortactin at a conserved threonine (T24) adjacent to the canonical Arp2/3 binding motif. Phosphorylation of cortactin T24 by CK2 $\alpha$ impairs the ability of cortactin to bind Arp2/3 and activate actin nucleation. Decreased invadopodia activity is observed in HNSCC cells with expression of CK2 $\alpha$ phosphorylation-null cortactin mutants, shRNA-mediated CK2 $\alpha$ knockdown, and with the CK2 $\alpha$ inhibitor Silmitasertib. Silmitasertib inhibits HNSCC collective invasion in tumor spheroids and orthotopic tongue tumors in mice. Collectively these data suggest that CK2 $\alpha-$ mediated cortactin phosphorylation at T24 is critical in regulating cortactin binding to Arp2/3 complex and pro-invasive activity, identifying a potential targetable mechanism for impairing HNSCC invasion. 
Implications: This study identifies a new signaling pathway that contributes to enhancing cancer cell invasion. 


\section{Introduction}

Cell invasion from the primary tumor is responsible for initiating the metastatic cascade and increasing cancer lethality $(1,2)$. Invasion is initiated in part through the action of invadopodia, actin-based membrane protrusions produced by tumor cells that mediate dissemination by degrading restrictive extracellular matrix (ECM) proteins through enzymatic matrix metalloproteinase (MMP) activity (3). Invadopodia contain a central filamentous (F-) actin core surrounded by an integrin-based adhesion ring complex that anchors the structure to allow focal matrix degradation and tumor cell protrusion through the basement membrane (4). Cortactin and actin-related protein (Arp) 2/3 complex are essential protein components involved in invadopodia precursor core formation required for subsequent MMP recruitment and membrane protrusion (5,6). Cortactin overexpression is common in several cancer types including head and neck squamous cell carcinoma (HNSCC), resulting in enhanced motility, invasion, and invadopodia activity $(7,8)$. Cortactin binding to Arp2/3 complex activates Arp2/3 actin nucleation activity, enhancing cellular actin polymerization to form branched F-actin networks $(6,8-$ 10). Cortactin also directly binds F-actin and bundles newly-formed filaments, providing an overall stabilizing effect on the Arp2/3-F-actin network required for invadopodia formation $(5,6,11)$. Previous work has shown that a DDW motif within the cortactin Nterminal acidic (NTA) domain is central in mediating Arp2/3 activation and branched actin network formation $(7,12-14)$. This region is similar to the Arp2/3 binding motif found in the Acidic region of the Verprolin, Central, Acidic (VCA) domain of the Wiskott-Aldrich Syndrome protein (WASp)-family of Arp2/3 nucleation promotion factors (NPFs). While the cortactin DDW motif is well established as the region responsible for Arp2/3 binding, 
post-translational or other modifications of amino acids in the NTA region that regulate binding have not been reported. Tyrosine and serine phosphorylation of cortactin residues in the carboxyl-terminal region are essential for invadopodia formation, cellular invasion, and tumor metastasis through multiple mechanisms ultimately involving activation of WASp NPFs $(6,7,15-18)$. In addition, comprehensive phosphorylation site mapping by mass spectroscopy has identified NTA phosphorylation sites in close proximity to the DDW motif (19). This raises the possibility that phosphorylation of one or more of these residues may serve to govern Arp2/3 binding and invadopodia function in invasive cancer.

Casein kinase (CK) 2 is a ubiquitously expressed, constitutively active serine/threonine kinase consisting of two catalytic subunits ( $\alpha$ or $\alpha^{\prime}$ ) and two $\beta$ regulatory subunits (20). Increased CK2 expression correlates with cell cycle progression, apoptosis resistance and tumor cell motility in various cancers (20). Overexpressed CK2 enhances HNSCC tumor cell motility (20). CK2 phosphorylates the cortactin homologue HS1 at an unidentified site(s) in the NTA region (21), as well as residues near the DDW region in the NPFs N-WASp and WAVE2 (22-25). Here we show that CK2 phosphorylation of threonine (T) 24 in the cortactin NTA impairs binding to and activation of Arp2/3 complex. Cortactin T24 and CK2 are required for efficient invadopodia formation and ECM degradation activity in HNSCC cell lines. Treatment of established and primary HNSCC cells with the selective CK2 inhibitor Silmitasertib impairs invadopodia function and regional HNSCC invasion. These results identify a new mechanism of invadopodia regulation that can be targeted to impair HNSCC invasion. 


\section{Materials and Methods}

\section{Cell culture, lentiviral infection and transfection, siRNA}

HNSCC cell lines OSC19 and UMSCC1 were acquired and maintained as described (26). MDA1586 cells were obtained in March 2014 from Barbara Frederick (University of Colorado, Denver, CO). All HNSCC lines were authenticated by STR profiling at the University of Arizona Genetics Core in June 2017. PCR-based mycoplasma testing (13100-01, Southern Biotech) was conducted on OSC19 and UMSCC1 lines in March 2015 and were free of contamination. The MDA1586 line was not tested for mycoplasma. HEK293T/17 cells were obtained in April 2013 from Robert Wysolmerski (West Virginia University, Morgantown, WV). NIH3T3 cells were obtained in June 2017 from Ivan Martinez (West Virginia University, Morgantown, WV) and maintained for $\leq 10$ passages. These lines were not authenticated or tested for mycoplasma. Cells were propagated in DMEM supplemented with $10 \%$ fetal bovine serum and $1 \%$ penicillin-streptomycin for $<6$ months. OSC19 and UMSCC1 cells stably infected with pLKO.1-puro cortactin shRNA or CK2 $\alpha$ shRNA were generated by clonal puromycin selection following standard methods (CSNK2A1: TRCN0000380839, TRCN0000027627; CTTN: TRCN0000040275).

UMSCC1 cells stably infected with pLU-Luc2 expressing luciferase were generated following standard methods. Murine cortactin rescue OSC19 and UMSCC1 cells containing cortactin shRNA stably infected with pLenti CMV Hygro cortactin constructs were generated by subsequent clonal hygromycin selection. Complete cortactin knockdown in OSC19 and UMSCC1 cells was achieved by transfection of cortactin- 
targeting siRNA (ON-TARGETplus SMARTpool L-010508-00-0020, Dharmacon) using a Nucleofector I (Amaxa Biosystems).

\section{Western blotting, antibodies and immunoprecipitation}

Western blotting was conducted as described (27) and visualized with autoradiography film (E3012, Denville Scientific) or captured by an Amersham Imager 600 (GE Healthcare Bio-Sciences). Antibodies used were: anti-cortactin clone 4F11 (1 $\mu \mathrm{g} / \mathrm{ml},(26))$, antipS473 AKT (\#4060, 1:1000; Cell Signaling Technology), anti-panAKT (\#2920, 1:1000; Cell Signaling Technology), anti- $\beta$-actin (\#8457, 1:1000; Cell Signaling Technology), antiCK2a (\#2656, 1:500, Cell Signaling Technology), anti-DYKDDDK (FLAG) clone 2EL1B11 (MAB3118, 1:500, Millipore) and anti-Arp3 (\#07-272, 1:500, EMD Millipore). Immunoprecipitation was conducted from cells lysed in 50mM Tris Buffer pH 8.0 with 10mM EDTA and 1\% NP-40 (28). Clarified lysates $(1 \mathrm{mg})$ were incubated with $50 \mu \mathrm{l}$ of FLAG M2 affinity resin (A2220, Sigma-Aldrich) for 2 hours at $4^{\circ} \mathrm{C}$. Immune complexes were collected by centrifugation, washed twice with Tris buffer, separated by SDS-PAGE, and Western blotted with antibodies as described above.

\section{Gelatin degradation assay, invadopodia characterization and microscopy}

Cells were plated on Oregon Green 488-conjugated gelatin (G13186, Invitrogen) coated coverslips (29). In cases of inhibitor treatment, cells were allowed to attach for 1 hour, then incubated for 12 or 24 hours with Silmitasertib (S2248, Selleckchem) as indicated. Cells were rinsed in PBS, fixed with 10\% buffered formalin (SF100-4, Fisher) and labeled as described (29). Antibodies used were 4F11 (1:500) or anti-FLAG (1:500). Primary antibodies were visualized using Alexa Fluor 647 conjugated goat anti-mouse secondary 
antibody (A21235, 1:500, Invitrogen). F-actin was visualized with rhodamine-conjugated phalloidin (R415, 1:1000, Invitrogen). Coverslips were mounted using ProLong Gold antifade with DAPI (P36935, Invitrogen). Images for quantifying gelatin degradation and knockdown/rescue expression were acquired with a Zeiss Axio Imager Z2 epifluorescent microscope equipped with an AxioCam MRm CCD camera and AxioVision software using LD Plan-Neofluar 40X/0.6 Corr and Plan-Apochromat 63X/1.4 oil objectives (Carl Zeiss Microscopy). Acquisition parameters were held constant within comparison groups. Confocal images were acquired using a Zeiss Axio Imager Z1 LSM510 confocal microscope with EC Plan-Neofluar 40X/1.30 and Plan-Apochromat 63X/1.4 oil objectives and Zen2009 software (Carl Zeiss Microcopy). All representative images were level adjusted to enhance contrast and brightness as needed and resized using Photoshop CC 2018 (Adobe Systems). Gelatin images were corrected for uneven illumination via bandpass filtering using ImageJ software $(\mathrm{NIH})$. Degradation and invadopodia formation was quantified as described previously (29), with $n \geq 70$ lentiviral infected or $\geq 100$ inhibitor-treated cells evaluated for each condition. FLAG-stained control images were thresholded against non-specific staining using ImageJ software. Cells above threshold values were considered positive for rescue construct expression and used for quantitation. For therapeutic treatments and RNAi stable cell lines, degradation and cell areas were determined by Image $\mathrm{J}(\mathrm{NIH})$ on an individual cell basis. Data represent the mean values normalized to control degradation area per cell area from at least 3 independent experiments. Invadopodia precursors were determined by colocalization of actin and cortactin at sites lacking gelatin degradation. Active invadopodia were determined by colocalization of actin, cortactin, and gelatin degradation. Data represent 
the mean from at least 3 independent experiments. Phase contrast images were acquired using a Zeiss Axiovert 200M microscope equipped with an AxioCamMR CCD camera using a Plan-Neofluar 10X/0.30 objective and AxioVision software (Carl Zeiss Microscopy).

\section{CK2 $\alpha$ kinase assay}

In vitro kinase assays were performed as described (30). Briefly, $0.25,0.5$, or $1 \mu \mathrm{g}$ of purified GST-WT or T24A cortactin NTA fusion proteins were incubated with 8 ng CK2 $\alpha$ (\#14-445, Millipore) and $10 \mu \mathrm{Ci} 32 \mathrm{Py}-\mathrm{ATP}$ (\#NEG002A500UC, PerkinElmer) at $30^{\circ} \mathrm{C}$ for 10 minutes. Reactions were terminated with hot SDS sample loading buffer. Proteins were visualized by autoradiography. Purified N-WASp GST-VCA $(0.5 \mu \mathrm{g})$ and GST $(1 \mu \mathrm{g})$ were used as respective positive and negative controls.

\section{In vitro cortactin phosphorylation binding assay}

Purified WT or T24A cortactin proteins $(2.5 \mu \mathrm{g})$ were bound to $4 \mathrm{~F} 11$-conjugated protein G magnetic beads (\#10003D, Life Technologies). Immune complexes were incubated in the presence or absence of activated CK2a (75 ng; \#V4482, Promega) and ATP (500 nmoles, \#BP413-25, Fisher Scientific) at $30^{\circ} \mathrm{C}$ for 15 minutes. Reactions were washed twice with $10 \mathrm{mM}$ Tris $\mathrm{pH} 7.4,150 \mathrm{mM} \mathrm{NaCl}, 0.5 \mathrm{mM}$ EDTA. Complexes were washed once with $10 \mathrm{mM}$ Tris $\mathrm{pH} 7.4,10 \mathrm{mM}$ EDTA and incubated with $50 \mathrm{ng}$ Arp2/3 complex (\#RP01A, Cytoskeleton) at $4^{\circ} \mathrm{C}$ for 30 minutes. Following incubation, binding complexes were washed once with 10mM Tris Buffer pH 7.4 with $25 \mathrm{mM} \mathrm{NaCl}$, 10mM EDTA, 1\% NP-40, then boiled and Western blotted with antibodies against cortactin and Arp3. 


\section{Actin polymerization assay}

Actin polymerization experiments were conducted as described previously (31). Reactions contained $2 \mu \mathrm{M}$ actin (10\% pyrene-labeled), $75 \mathrm{nM}$ Arp2/3 complex, $100 \mathrm{nM}$ cortactin or $50 \mathrm{nM} \mathrm{GST-VCA} \mathrm{( \# VCG03,} \mathrm{Cytoskeleton),} \mathrm{and/or} \mathrm{varying} \mathrm{amounts} \mathrm{of} \mathrm{CK2} \alpha$ (\#14-445, Millipore) as indicated. For reactions with CK2a, GST-VCA or cortactin mutants were preincubated with CK2 $\alpha$ and 500 nmoles unlabeled ATP for 15 minutes at room temperature prior to addition to the actin polymerization reaction.

\section{PDX-derived cell lines}

Patient-derived xenograft (PDX) tumors and cell lines were established as described (32). WVUSCC-AR2 and WVUSCC-AR5 were derived from surgical specimens of alveolar ridge HNSCC in compliance with West Virginia University Institutional Review Board approved protocol \#1310105737A033. PDXs were developed in compliance with West Virginia University Institutional Animal Care and Use Committee approved protocol \#150302.6 by placing approximately $1 \mathrm{~mm}$ tumor fragments into subcutaneous pockets in the flanks of anesthetized 8-10 week old NOD/SCID-y (NSG) mice. Tumor fragments were overlayed with Matrigel (354234, Corning) and incisions were closed using wound clips. Mice were weighed and monitored for tumor growth on a weekly basis. PDX tumors were passed into new NSG mice and/or used to generate cell lines once tumors reached $\sim 1$ $\mathrm{cm}$ in greatest dimension.

For cell line derivation, PDX tumors were minced and digested in DMEM supplemented with $20 \%$ FBS and $1 \mathrm{mg} / \mathrm{mL}$ collagenase IV (17104019, Gibco). Digested tissues were 
plated onto NIH 3T3 fibroblasts senesced with $4 \mu \mathrm{g} / \mathrm{mL}$ mitomycin C (BP2531, Fisher) and cultured in DMEM:F12 1:1 supplemented with 10\% FBS, $400 \mathrm{ng} / \mathrm{mL}$ hydrocortisone (H0888, Sigma), $50 \mu \mathrm{g} / \mathrm{mL}$ gentamycin (15750060, Gibco), $5 \mu \mathrm{M}$ ROCK inhibitor (S1049, Selleckchem), $0.5 \mathrm{ng} / \mathrm{mL}$ recombinant human epidermal growth factor (EGF) (PHG0311, Gibco), and $10 \mathrm{ng} / \mathrm{mL}$ cholera toxin (C8062, Sigma). Both WVUSCC-AR2 and -AR5 were derived in August 2017 and maintained for $\leq 10$ passages. Derived lines were verified using cytokeratin 14 staining (ab15462, Abcam). Neither STR profiling nor mycoplasma detection was performed on these cell lines. Prior to utilization in gelatin degradation or spheroid invasion assays, PDX derived cell lines were plated directly onto cell culture

dishes for 1-2 passages to remove the fibroblast population. Gelatin degradation and spheroid invasion assays were performed in DMEM supplemented with 10\% FBS.

\section{In vitro tumor spheroid invasion}

3D spheroid invasion assays were performed as previously described (26). 1 $1 \times 10^{4}$ (OSC19) or 2.5 $\times 10^{4}$ (UMSCC1 and WVUSCC-AR5) cells were plated into individual wells coated with $1.5 \%$ noble agar for $24 \mathrm{~h}$ (UMSCC1) or $48 \mathrm{~h}$ (OSC19 and WVUSCC-AR5) to form spheroids. For each line, spheroids were collected, resuspended in $500 \mu \mathrm{L}$ of 2 $\mathrm{mg} / \mathrm{mL}$ rat tail collagen I (354236, Corning), and plated into individual wells of a 24-well plate pre-coated with $400 \mu \mathrm{L}$ solidified $2 \mathrm{mg} / \mathrm{mL}$ collagen I. Plates were incubated for $1 \mathrm{~h}$ at $37^{\circ} \mathrm{C}$, then overlayed with $1 \mathrm{~mL}$ DMEM supplemented with $10 \%$ fetal bovine serum and $1 \%$ penicillin-streptomycin containing DMSO or $10 \mu \mathrm{M}$ Silmitasertib. Spheroid invasion was visualized at the indicated time points by phase contrast microscopy using a Zeiss Axiovert 200M microscope equipped with an AxioCamMR CCD camera using a Plan- 
Neofluar 5X/0.15 objective and Axiovision software (Carl Zeiss Microscopy). Maximal radial distances for invaded cells were calculated using Axiovision software, with invasive distance determined as the difference between the initial and final maximum radius for each invaded spheroid.

\section{Orthotopic tongue tumors and invasion analysis}

Tongue tumor establishment was adapted from previous work (33). $2.5 \times 10^{4}$ luciferase expressing UMSCC1 cells were injected into the tongues of 8-10 week old NSG mice (purchased from the West Virginia University Transgenic Animal Core Facility). Mice were maintained using transgenic dough diet (S3472, Bioserve) and weighed every 2-3 days. Tumor growth was monitored by bioluminescent imaging using $150 \mathrm{mg} / \mathrm{kg}$ D-luciferin (122796, Caliper Life Sciences) injected intraperitoneally, followed by in vivo whole-body bioluminescence imaging using an IVIS Lumina-II system and Living Image 4.0 software (PerkinElmer). Tumors were allowed to establish for one week, then mice were divided equally into two groups based on approximate tumor size. Mice were given $50 \mathrm{mg} / \mathrm{kg}$ Silmitasertib in DMSO or DMSO alone by oval gavage twice daily for three weeks. Mice were subsequently euthanized, tongues excised, processed and stained for histological analysis.

To quantify invasion parameters, whole tongue histological images were cropped to encompass the tumor invasive front and analyzed using ImageJ. Images were processed with the colour deconvolution 1.5 plugin using H\&E or H\&E2 presets. Resultant colour_1 images were $25 \%$ contrast enhanced before conversion into binary images. ROls were selected for particles above 15,000 pixel units and manually verified by overlay onto the 
original H\&E image to remove artifacts. Invasive protrusions were defined as projections at the leading edge of the tumor surrounded by stroma on three sides and identified on the binary image using the polygon selection tool. Invasive distance was determined as the difference between the farthest edge of the protrusion and the protrusion base.

\section{Statistical analysis}

Differences in mean values between groups were evaluated using Student's or Welch's $t$ test. Significance was determined at $p<0.05$ utilizing GraphPad Prism 7 software. Error bars represent +/- S.E.M. 


\section{Results}

\section{Cortactin threonine 24 is required for Arp2/3 complex binding and activation}

Phosphorylation of serine (S) 11, T13, and T24 in the murine cortactin NTA domain has been reported (19). The proximity of these residues to the canonical Arp2/3 binding motif consisting of amino acids 20-22 (DDW) has the potential to regulate Arp2/3 binding (Fig. 1A). To determine if these residues influence cortactin binding to Arp2/3, FLAG-tagged murine cortactin constructs were generated that contained serine to alanine (S11A) and threonine to alanine (T24A) phosphorylation-null mutations. T13 was not evaluated since it is not conserved in human cortactin. Co-immunoprecipitation studies indicate that S11A cortactin bound endogenous Arp2/3 at levels similar to wild-type (WT) cortactin, while T24A cortactin failed to effectively bind Arp2/3 despite retaining the DDW binding motif (Fig. 1B). Threonine to aspartic acid (T24D) phosphomimetic cortactin bound Arp2/3 at reduced levels compared to WT (Fig. 1B). These data demonstrate that both the DDW motif and T24 are required for optimal Arp2/3 complex binding. Furthermore, reduced Arp2/3 binding resultant from the addition of negative charge at amino acid 24 (T24D) suggests that phosphorylation may play a negative-regulatory role.

To assess the impact of T24 on Arp2/3 actin nucleation, recombinant human WT, $\Delta \mathrm{DDW}$, and T24A cortactin proteins were expressed in bacteria and purified (Fig. 1C). When evaluated in pyrene-labeled actin assembly assays, WT cortactin displayed slower polymerization kinetics compared to the neuronal (N)-WASp VCA domain, whereas the $\triangle \mathrm{DDW}$ mutant failed to activate Arp2/3 as previously reported (Fig. 1D, (16,34,35)). T24A cortactin demonstrated intermediate activity, with reduced nucleation levels compared to 
WT cortactin and increased nucleation compared to $\triangle \mathrm{DDW}$ (Fig. 1D). Taken together these data identify T24 in the cortactin NTA as a critical residue required for optimal cortactin-mediated Arp2/3 binding and activation regardless of phosphorylation status.

\section{Cortactin T24 is required for invadopodia-mediated ECM degradation}

Cortactin is essential for initiating invadopodia formation, maturation, and ECM degradation in part due to NTA-mediated Arp2/3 binding $(36,37)$. To determine the role of cortactin T24 in invadopodia function, a panel of cortactin knockdown-rescue cell lines stably expressing FLAG-cortactin mutant constructs were produced in invasive UMSCC1

(Fig. 2) and OSC19 (Supplementary Fig. S1) HNSCC cell lines. Both lines spontaneously produce invadopodia and degrade ECM $(26,38)$. While individual cortactin siRNA (siCTTN) and shRNA (shCTTN) treatment resulted in decreased cortactin expression and matrix degradation in each case (Fig. 2D, Supplementary Fig. S1B, Supplementary Fig. S2), sequential exposure to cortactin siRNA in stable shRNA cells resulted in efficient and reliable cortactin knockdown (KD; Fig. 2, Supplementary Fig. S1, Supplementary Fig. S2). Cortactin KD cells were used for subsequent experimentation to minimize the possibility of residual endogenous cortactin masking the effects of re-expressed FLAG-cortactin mutants. FLAG-WT cortactin expression in KD cells partially restored the amount of active invadopodia formation in UMSCC1 cells (Fig. 2A \& C) and fully restored ECM degradation in UMSCC1 (Fig. 2A \& B) and OSC19 (Supplementary Fig. S1A \& C) cell lines. FLAG$\triangle D D W$ enhanced invadopodia precursor formation but failed to rescue active invadopodia and ECM degradation (Fig. 2A-C, Supplementary Fig. 1A \& C). Similarly, both FLAGT24A and FLAG-T24D cortactin restored invadopodia precursor formation while failing to induce invadopodia maturation above KD levels, with active invadopodia and ECM 
degradation levels for both mutants similar to that of FLAG- $\triangle$ DDW cortactin (Fig. 2A-C, Supplementary Fig. S1A, C, D). These results suggest that Arp2/3 binding and activation facilitated by cortactin T24 is required for effective cortactin-mediated invadopodia formation and ECM degradation in HNSCC cells.

\section{CK2 phosphorylation of cortactin T24 regulates interaction with Arp2/3 complex}

The importance of T24 in Arp2/3 activation and invadopodia function, along with prior identification of T24 as a cortactin phosphorylation site, led us to identify the kinase(s) responsible for phosphorylating T24. Computational analysis of the sequences flanking T24 was performed by seven independent predictive algorithms, six of which suggested that CK2 $\alpha$ had the highest probability of phosphorylating cortactin T24 (Supplementary Table S1). To test this, kinase assays were conducted using GST-tagged cortactin WT and T24A NTA fusion proteins with purified active CK2 $\alpha$. The N-WASp VCA domain was used as a positive control, since previous studies have shown this region to be a CK2 $\alpha$ substrate $(23,25)$. Increasing amounts of GST-WT-NTA were efficiently phosphorylated by CK2 $\alpha$, whereas no phosphorylation was evident in GST-T24A-NTA (Fig. 3A). These data indicate that cortactin T24 can serve as a CK2 $\alpha$ substrate, and that T24 is the only residue targeted by CK2 $\alpha$ in the NTA region.

To determine if CK2 $\alpha$ phosphorylation of cortactin T24 effects binding to Arp2/3 complex, recombinant human WT or T24A cortactin proteins were pre-incubated with or without CK2 $\alpha$, then mixed with purified Arp2/3. Phosphorylation of WT cortactin by CK2 $\alpha$ reduced binding of Arp2/3 complex to background levels (beads alone) whereas no impact on T24A was observed (Fig. 3B). To ascertain the impact of CK2 $\alpha$ phosphorylation on 
cortactin-mediated Arp2/3 activation, actin assembly assays were conducted with CK2 $\alpha$ phosphorylated cortactin and N-WASp VCA domain. As previously determined, CK2 $\alpha$ phosphorylation of N-WASp VCA results in a modest reduction of Arp2/3 NPF activity (black vs. grey, Fig. 3C, (25)). Similarly, WT cortactin incubated with increasing amounts of CK2 $\alpha$ prior to inclusion in polymerization assays resulted in a dose dependent suppression of actin assembly, suggesting that CK2 $\alpha$ phosphorylation impairs the ability of cortactin to activate Arp2/3 complex (Fig. 3C). Although Arp2/3 can be activated by direct phosphorylation from multiple kinases (39-41), CK2 $\alpha$ had no direct effect on Arp2/3 activation (orange vs. pale green, Fig. $3 C$ ), suggesting that the inhibitory effect on Arp $2 / 3$ activity is due to phosphorylated cortactin in these assays. To determine if the CK2 $\alpha-$ targeted T24 residue is responsible for the observed inhibitory effect on Arp2/3 activity, polymerization assays were conducted with WT and T24A cortactin proteins following incubation with CK2 $\alpha$. While CK2 $\alpha$ inhibited the ability of WT cortactin to activate Arp2/3 complex (blue vs. red, Fig. 3D), preincubation of CK2 $\alpha$ with T24A cortactin exhibited no additional inhibitory effect on Arp2/3 nucleation activity (green vs. purple, Fig. 3D). Collectively these data indicate that cortactin phosphorylation at T24 by CK2 $\alpha$ reduces the ability of cortactin to bind and activate Arp2/3 complex-mediated branched actin network formation.

\section{CK2 $\alpha$ is required for optimal HNSCC invadopodia function}

To determine if CK2 $\alpha$ impacts invadopodia function, CK2 $\alpha$ expression was stably knocked down in OSC19 and UMSCC1 cells using anti-CK2 $\alpha$ shRNAs targeting two different regions in the CK2 $\alpha$ transcript. Both shRNAs reduced CK2 $\alpha$ expression to nondetectable levels in each cell line (Fig. 4A). Neither line expressed the alternative CK2 $\alpha^{\prime}$ 
isoform in control or CK2 $\alpha$ knockdown cells (data not shown). CK2 $\alpha$ knockdown in OSC19 cells reduced the level of active invadopodia by $71-88 \%$ and ECM degradation by $63-$ $73 \%$, whereas knockdown in UMSCC1 cells reduced active invadopodia formation by 68 $92 \%$ and ECM degradation by $36-60 \%$ (Fig. 4B-D). While these data indicate that CK2 $\alpha-$ is a key mediator of invadopodia maturation and function, neither invadopodia formation nor matrix degradation was entirely abolished. This would indicate that alternative signaling pathways impinging on cortactin and other invadopodia proteins remain active in the absence of CK2 $\alpha$ expression.

\section{The CK2 $\alpha$ inhibitor Silmitasertib suppresses invadopodia function in HNSCC cells}

Silmitasertib (CX-4945) is an orally bioavailable small molecule ATP-competitive inhibitor that targets CK2 $\alpha$ kinase activity and is currently undergoing clinical trials in multiple cancer types (20) (NCT01199718, NCT02128282, NCT00891280). To determine the impact of Silmitasertib on HNSCC tumor cell-mediated invadopodia formation and ECM degradation, established HNSCC cell lines were treated with increasing Silmitasertib concentrations and evaluated for effects on invadopodia activity and ECM degradation. Dose-dependent decreases in ECM degradation were observed at concentrations above $0.5 \mu \mathrm{M}$ in all evaluated HNSCC lines (Fig. 5A \& B). The greatest impairment of gelatin degradation was seen at $10 \mu \mathrm{M}$ (Fig. 5A \& B), comparable to effective CK2 specific growth-inhibitory doses in several cancer cell lines $(20,42)$. At this concentration, active invadopodia formation was significantly diminished in OSC19 and UMSCC1 cells, whereas MDA1586 cells displayed non-significant decreases (Fig. 5C). To determine if the invadopodia inhibitory effect of Silmitasertib was directly due to altering CK2amediated cortactin T24 phosphorylation, antibodies against phosphorylated cortactin T24 
peptides ( $\mathrm{pT} 24$ ) were designed and purified. Attempts by two different commercial vendors failed to generate a pT24-specific antibody (not shown). Therefore, we evaluated the phosphorylation status of S473 (pS473) in AKT following Silmitasertib treatment as a surrogate marker for drug efficacy, as this site is known to be phosphorylated by CK2 $\alpha$ (20). MDA1586 cells treated with 1 or $10 \mu \mathrm{M}$ Silmitasertib had decreased pS473 AKT after 24 hours, the same timeframe used in ECM degradation assays (Supplementary Fig. S3). OSC19 and UMSCC1 cells had decreased pS473 AKT after treatment with 10 $\mu \mathrm{M}$ Silmitasertib for 12 hours, the time used for matrix degradation assays in these lines (Supplementary Fig. S3).

The fact that CK2 $\alpha$ phosphorylates multiple targets aside from cortactin (20) raises the possibility that the inhibitory effect of Silmitasertib may be due to impairing phosphorylation of additional proteins involved in invadopodia function. To determine the extent of cortactin-specific CK2 $\alpha$ phosphorylation in invadopodia-mediated ECM degradation, UMSCC1 cells expressing FLAG-WT, -T24A, and -T24D cortactin were treated with $1 \mu \mathrm{M}$ Silmitasertib and evaluated for additional suppressive effects on matrix degradation (Fig. 5D \& E). Silmitasertib diminished ECM degradation in cells expressing FLAG-WT cortactin by $51 \%$, similar to the reduction observed in non-transfected cells (Fig 5D vs B). Neither FLAG-T24A or FLAG-T24D expressing cells treated with Silmitasertib demonstrated reductions in ECM degradation levels from baseline vehicletreated controls (Fig. 5D \& E). While these results do not entirely negate alternative CK2dependent signaling pathways in invadopodia regulation, it does suggest that cortactin T24 is the primary CK2 $\alpha$ target in governing HNSCC invadopodia function. 
To evaluate the anti-invadopodia effect of Silmitasertib in more translationally-relevant models, patient-derived xenografts (PDXs) were derived from a well- and a moderatelydifferentiated HNSCC surgical sample. PDX tumors maintained original patient tumor architecture, displaying collective invasion and keratin pearls characteristic of differentiated HNSCC (Fig. 6A). Primary cell lines derived from these PDX tumors form invadopodia and spontaneously degrade gelatin within 24 hours (Fig. 6B). Both lines exhibit tight colony morphology under cell culture conditions consistent with HNSCC lines derived from epithelial HNSCC (Fig. 6B, $(32,43)$ ). Treatment of WVUSCC-AR2 and WVUSCC-AR5 with Silmitasertib yielded similar results to those observed in established lines, with gelatin degradation impaired $38-62 \%$ in WVUSCC-AR2 cells and $56-66 \%$ in WVUSCC-AR5 cells at and above $0.5 \mu \mathrm{M}$ (Fig. 6C \& D). Similarly, $10 \mu \mathrm{M}$ Silmitasertib treatment suppressed active invadopodia by $76 \%$ in WVUSCC-AR2 and $91 \%$ in WVUSCC-AR5 (Fig. 6E). Collectively these data indicate that CK2 $\alpha$ kinase activity is essential for maximal ECM degradation ability in HNSCC.

\section{Silmitasertib inhibits HNSCC invasion}

To determine if Silmitasertib impacts HNSCC invasion, we initially utilized a 3D in vitro assay designed to model collective invasion typically observed in differentiated HNSCC. Tumor spheroids generated from OSC19, UMSCC1 and WVUSCC-AR5 PDX lines were embedded between layers of collagen I. WVUSCC-AR2 cells failed to form spheroids and could not be used in this assay. Spheroids were treated with $10 \mu \mathrm{M}$ Silmitasertib or vehicle (DMSO) for $48 \mathrm{~h}$ (Fig. 7A \& B). Silmitasertib significantly reduced 3D collective invasion in all assayed lines (Fig. 7B). We next evaluated the ability of Silmitasertib to control invasion in the tongues of mice harboring orthotopic tumors. Luciferase-expressing 
UMSCC1 cells injected into the tongues of NSG mice formed detectable tumors within one week (Fig. 7C). Mice were divided into two groups containing similar tumor size as determined by in vivo bioluminescence, with one group treated with vehicle and one with Silmitasertib. Mice dosed twice daily for three weeks displayed a non-significant reduction in tumor growth compared to controls, similar to previous single agent xenograft studies (Fig. 7C, (20)). To negate potential bias due to unequal tumor size, four equivalent tumors from each group were selected for further assessment. In-depth evaluation of tumor margins from tongues excised after four weeks revealed alterations in invasive characteristics of Silmitasertib-treated mice (Fig. 7D-I). Tumors in drug-treated mice had a less ragged appearance at the invasive front, exhibiting shorter and smaller collective cell protrusions into the tongue stroma compared to control mice (Fig. 7D-G). In addition, tongues from Silmitasertib-treated mice had reduced perineural invasion of nerves adjacent to the invasive front (Fig. $7 \mathrm{H} \& \mathrm{I}$ ). No difference was seen in the size or invasive distance of detached collective groups within the invasive front (Supplementary Fig. S4). Collectively these data support a role for CK2 $\alpha$ signaling in driving several pro-invasive behaviors associated with poor patient outcome in HNSCC $(2,44)$.

\section{Discussion}

Proteolysis of restrictive tissue barriers is essential to all steps in the metastatic cascade, and increasing evidence indicates that the proteolytic activity of invadopodia is required for invasive breaching of ECM barriers (1). Signals that govern invadopodia are dynamic and highly regulated, requiring coordinated activity of several oncogenic pathways in parallel to achieve maximal efficiency (1). Active assembly and turnover of cellular F-actin 
networks is required for initiating invadopodia formation and subsequent maturation, involving recruitment and activation of membrane-bound and secreted MMPs to mediate ECM proteolysis (1). Breakdown of existing F-actin networks, in conjunction with Arp2/3 activation in invadopodia, is responsible for actin network turnover necessary for productive branched F-actin formation that drives membrane protrusion. Early recruitment of cortactin is necessary for invadopodia initiation, where phosphorylation of C-terminal tyrosines 421,470 , and 486 , along with serines 405 and 418 , occurs downstream of growth factor and integrin signaling (45). These phosphorylation events create binding sites for scaffolding platforms that recruit N-WASp and WAS protein family member 2 (WASF2; WAVE2, WASp family Verprolin-homologous protein 2), ensuring that activation of Arp2/3 is maintained throughout the invadopodia cycle (6). Cortactin also recruits the F-actin severing protein cofilin to invadopodia, providing the necessary machinery for cyclic actin network breakdown and regrowth during invadopod extension (16). In addition to these important C-terminal functions, the ability of the NTA region to directly bind Arp2/3 is also essential for invadopodia formation and ECM degradation, presumably through direct Arp2/3 activation and prolonged stabilization of Arp2/3-F-actin networks $(10,16)$.

Regarding the ability of the cortactin NTA to bind Arp2/3, this study reveals two distinct findings. First, that T24 is required for Arp2/3 binding, since mutation of this residue ablates (A) or reduces (D) the association of cortactin with $A r p 2 / 3$ in coimmunoprecipitation assays. T24 is two residues C-terminal to the well-defined DDW Arp2/3 interaction motif that is conserved in other Arp2/3 NPFs (6). This places T24 in close proximity to contribute to Arp2/3 binding. While the crystal structure of the cortactin 
NTA bound to Arp2/3 complex has not been reported, previous chemical crosslinking and three-dimensional reconstruction studies indicate that the NTA region primarily binds the Arp3 subunit in the complex, with the most $\mathrm{N}$-terminal residues spanning the Arp2-Arp3 interface to contact the Arp2 subunit (Graphical abstract, $(9,34)$ ). Thus, T24 may contribute essential hydrogen bonding with recipient polar residues near the basic side chains on Arp3 involved in electrostatic binding to the acidic NTA residues. This concept is supported by the requirement of W22 in the DDW motif for Arp2/3 binding, along with the lack of an equivalent threonine in the VCA region of WASp proteins $(16,34,35)$. While T24A cortactin appears incapable of binding Arp2/3, it can activate Arp2/3 actin nucleation in conditions where $\triangle \mathrm{DDW}$ cortactin remains inactive (Fig. 1D). This may be due to T24 having a lower affinity for Arp3 than the DDW region and/or the high cortactin molar levels required to stimulate NPF activity in vitro. Nonetheless, the requirement for cortactin T24 in invadopodia-mediated ECM degradation supports an essential biological role for this residue in a non-phosphorylated context.

Secondly, our study shows that T24 phosphorylation serves to negatively regulate cortactin binding to and activation of Arp2/3 complex (Fig. 3B-D). Addition of the phosphate group to T24 likely imparts a steric and electrostatic disruption, preventing the DDW and other interacting residues in the NTA from initiating and/or maintaining binding to Arp3. Steric interference may be the predominant mechanism, since T24D cortactin can bind Arp2/3 at reduced levels (Fig. 1B), indicating that negative charge alone is insufficient to completely prevent Arp2/3 association. Phosphorylation of T24 therefore serves to block the ability of cortactin to bind Arp2/3, similar to the regulation of the Arp2/3F-actin regulatory proteins coronin $1 \mathrm{~B}$ and cofilin $(46,47)$. While we did not determine if 
phosphorylation of T24 serves to release cortactin from existing Arp2/3-F-actin branchpoints, phosphorylation of Arp2/3-bound cortactin T24 may be an additional mechanism to dissociate cortactin from these regions. Such a mechanism could function in facilitating breakdown and recycling of invadopodia Arp2/3-F-actin networks.

The ability of CK2 $\alpha$ to phosphorylate cortactin T24 supports a wider role for this kinase in actin regulation. Previous work identified T23 as a putative but unconfirmed CK2 $\alpha$ phosphorylation site in the NTA region of myeloid-cell specific cortactin homolog HS1 (21). In addition, CK2 $\alpha$ phosphorylates two adjacent sites in the A domain of WASp family proteins (22-25). These studies report conflicting results on Arp2/3 activity, with phosphorylation of WASp enhancing and in N-WASp and WAVE2 inhibiting VCAstimulated Arp2/3-mediated actin polymerization. Our results with CK2 $\alpha$-phosphorylated N-WASp VCA domain also shows an inhibitory effect on Arp2/3 activation (Fig. 3C), suggesting that CK2 $\alpha$ effects may be differential to specific WASp family proteins.

Cortactin T24A and T24D expression in KD HNSCC lines impairs active invadopodia formation and subsequent ECM degradation (Fig. 2A-C, Supplementary Fig. 1A \& C), supporting in vitro evidence that T24 phosphorylation prevents Arp2/3 binding and activation necessary for invadopodia activity. The importance of CK2 $\alpha$-mediated cortactin T24 phosphorylation is further supported by the inability of Silmitasertib to further suppress ECM degradation in cortactin T24A or T24D expressing cells (Fig. 5D \& E). CK2a-specific knockdown or pharmacologic inhibition in cells would therefore be expected to reduce phosphorylation of cortactin and WASp family proteins, resulting in net increased Arp2/3 activity as observed from in vitro studies. The fact that CK2 $\alpha$ 
knockdown or kinase blockade also impairs invadopodia formation and function is paradoxical but can be explained by cyclic CK2 $\alpha$ phosphorylation and dephosphorylation to regulate cortactin binding to and activation of $A r p 2 / 3$ in invadopodia during maturation and elongation. Dephosphorylation of cortactin T24 and WASp NPFs by unknown phosphatases would enable these NPFs to promote Arp2/3 activation, actin polymerization, and invadopodia activity. Following network breakdown during actin turnover, CK2 $\alpha$ phosphorylation would return NPFs to the inactive state to await the next cycle of actin assembly. While similar cyclic regulation of Src and cofilin has been shown to be essential for invadopodia function and provides support for this model $(38,46)$, confirmation of T24 phosphorylation within invadopodia leading to altered actin dynamics along with identification of the T24 phosphatase are necessary to fully confirm this proposed mechanism.

Locoregional control of HNSCC dissemination is problematic, where perineural invasion and invasive metastatic spread to cervical lymph nodes accelerates patient decline $(44,48,49)$. Cortactin and CK2 $\alpha$ expression are elevated in HNSCC and individually correspond with poorer patient outcomes $(7,20)$. CK2 knockdown and Silmitasertib treatment in HNSCC cells has anti-proliferative and anti-metastatic properties in vitro and in mice, providing support for the utility of Silmitasertib in HNSCC (20). The anti-invasive effect of Silmitasertib shown here is likely due in part to disruption of the invadopodia actin assembly through combined inhibition of multiple Arp2/3 NPFs. We note that T24 cortactin mutants, CK2 $\alpha$ knockdown, and Silmitasertib treatment in HNSCC cells does not completely abolish invadopodia formation, degradation activity or invasion. Activation of invadopodial Arp2/3 NPFs through alternative scaffolding or lipid-based signaling 
pathways that can bypass CK2 inhibition can account for this residual activity (1). However, our results indicate that CK2 $\alpha$ inhibition by a single agent does significantly impair collective HNSCC invasion. Given the current paucity of treatment options for invasive HNSCC, combining Silmitasertib with additional drugs known to impair invadopodia function by blocking additional invadopodia and motility pathways (8) has the potential to provide novel treatment options for controlling invasive spread of late-stage HNSCC harboring elevated cortactin and/or CK2 expression.

\section{Acknowledgments}

We thank Jianjiong Gao (University of Missouri) for sequence prediction analysis. The assistance of the West Virginia University Microscope Imaging Facility, Animal Models and Imaging Facility, and Biochemistry Protein Core are gratefully acknowledged. 


\section{References}

1. Eddy RJ, Weidmann MD, Sharma VP, Condeelis JS. Tumor Cell Invadopodia: Invasive Protrusions that Orchestrate Metastasis. Trends Cell Biol. Elsevier Ltd; 2017;27:595-607.

2. Markwell S, Weed S. Tumor and Stromal-Based Contributions to Head and Neck Squamous Cell Carcinoma Invasion. Cancers (Basel). 2015;7:382-406.

3. Bowden ET, Barth M, Thomas D, Glazer RI, Mueller SC. An invasion-related complex of cortactin, paxillin and PKCmu associates with invadopodia at sites of extracellular matrix degradation. Oncogene. 1999;18:4440-9.

4. Branch KM, Hoshino D, Weaver AM. Adhesion rings surround invadopodia and promote maturation. Biol Open. 2012;1:711-22.

5. Schnoor M, Stradal TE, Rottner K. Cortactin: Cell Functions of A Multifaceted Actin-Binding Protein. Trends Cell Biol. 2018;28:79-98.

6. Yin M, Ma W, An L. Cortactin in cancer cell migration and invasion. Oncotarget. Impact Journals, LLC; 2017;8:88232-43.

7. MacGrath SM, Koleske AJ. Cortactin in cell migration and cancer at a glance. J Cell Sci. Company of Biologists; 2012;125:1621-6.

8. Meirson $\mathrm{T}$, Gil-Henn $\mathrm{H}$. Targeting invadopodia for blocking breast cancer metastasis. Drug Resist Updat. 2018;39:1-17.

9. Xu X-P, Rouiller I, Slaughter BD, Egile C, Kim E, Unruh JR, et al. Threedimensional reconstructions of Arp2/3 complex with bound nucleation promoting factors. EMBO J. Nature Publishing Group; 2012;31:236-47.

10. Weaver AM, Karginov A V., Kinley AW, Weed SA, Li Y, Parsons JT, et al. Cortactin promotes and stabilizes Arp2/3-induced actin filament network formation. Curr Biol. 2001;11:370-4.

11. Helgeson LA, Prendergast JG, Wagner AR, Rodnick-Smith M, Nolen BJ. Interactions with Actin Monomers, Actin Filaments and Arp2/3 Complex Define the Roles of WASP Family Proteins and Cortactin in Coordinately Regulating Branched Actin Networks. J Biol Chem. 2014;289:1-26.

12. Weed SA, Karginov A V., Schafer DA, Weaver AM, Kinley AW, Cooper JA, et al. Cortactin localization to sites of actin assembly in lamellipodia requires interactions with F-actin and the Arp2/3 complex. J Cell Biol. 2000;151:29-40.

13. Weaver AM, Young ME, Lee W-L, Cooper JA. Integration of signals to the Arp2/3 complex. Curr Opin Cell Biol. 2003;15:23-30.

14. Blanchoin L, Amann KJ, Higgs HN, Marchand J, Kaiser DA, Pollard TD. Direct observation of dendritic actin filament networks nucleated by Arp2/3 complex and WASP / Scar proteins. Nature. 2000;404:1007-11. 
15. Martinez-quiles N, Ho HH, Kirschner MW, Ramesh N, Geha RS. Erk / Src Phosphorylation of Cortactin Acts as a Switch On-Switch Off Mechanism That Controls Its Ability To Activate N-WASP. Mol Cell Biol. 2004;24:5269-80.

16. Oser M, Yamaguchi H, Mader CC, Bravo-Cordero JJ, Arias M, Chen X, et al. Cortactin regulates cofilin and N-WASp activities to control the stages of invadopodium assembly and maturation. J Cell Biol. 2009;186:571-87.

17. Mader CC, Oser M, Magalhaes MAO, Bravo-Cordero JJ, Condeelis J, Koleske AJ, et al. An EGFR-Src-Arg-Cortactin pathway mediates functional maturation of invadopodia and breast cancer cell invasion. Cancer Res. 2011;71:1730-41.

18. Ayala I, Baldassarre M, Giacchetti G, Caldieri G, Tetè S, Luini A, et al. Multiple regulatory inputs converge on cortactin to control invadopodia biogenesis and extracellular matrix degradation. J Cell Sci. 2008;121:369-78.

19. Martin KH, Jeffery ED, Grigera PR, Shabanowitz J, Hunt DF, Parsons JT. Cortactin phosphorylation sites mapped by mass spectrometry. J Cell Sci. 2006;119:2851-3.

20. Chua MMJ, Ortega CE, Sheikh A, Lee M, Abdul-Rassoul H, Hartshorn KL, et al. CK2 in Cancer: Cellular and Biochemical Mechanisms and Potential Therapeutic Target. Pharmaceuticals (Basel). Multidisciplinary Digital Publishing Institute (MDPI); 2017;10.

21. Ruzzene M, Brunati AM, Sarno S, Marin O, Donella-Deana A, Pinna LA. Ser/Thr phosphorylation of hematopoietic specific protein 1 (HS1): implication of protein kinase CK2. Eur J Biochem. 2000;267:3065-72.

22. Mendoza MC. Phosphoregulation of the WAVE regulatory complex and signal integration. Semin Cell Dev Biol. Elsevier Ltd; 2013;24:272-9.

23. Pocha SM, Cory GO. WAVE2 is regulated by multiple phosphorylation events within its VCA domain. Cell Motil Cytoskeleton. 2009;66:36-47.

24. Cory GOC, Cramer R, Blanchoin L, Ridley AJ. Phosphorylation of the WASP-VCA domain increases its affinity for the Arp2/3 complex and enhances actin polymerization by WASP. Mol Cell. 2003;11:1229-39.

25. Galovic M, Xu D, Areces LB, van der Kammen R, Innocenti M. Interplay between N-WASP and CK2 optimizes clathrin-mediated endocytosis of EGFR. J Cell Sci. 2011;124:2001-12.

26. Hayes KE, Walk EL, Ammer AG, Kelley LC, Martin KH, Weed SA. Ableson Kinases Negatively Regulate Invadopodia Function and Invasion in Head and Neck Squamous Cell Carcinoma by Inhibiting an HB-EGF Autocrine Loop. Oncogene. 2013;32:4766-77.

27. Ammer AG, Kelley LC, Hayes KE, Evans J V, Lopez-skinner A, Martin KH, et al. Saracatinib impairs head and neck squamous cell carcinoma invasion by disruption invadopodia function. J Cancer Sci Ther. 2009;1:52-61. 
28. Head JA, Jiang D, Li M, Zorn LJ, Schaefer EM, Parsons JT, et al. Cortactin Tyrosine Phosphorylation Requires Rac1 Activity and Association with the Cortical Actin Cytoskeleton. Mol Biol Cell. 2003;14:3216-29.

29. Martin KH, Hayes KE, Walk EL, Ammer AG, Markwell SM, Weed SA. Quantitative Measurement of Invadopodia-mediated Extracellular Matrix Proteolysis in Single and Multicellular Contexts. J Vis Exp. 2012;e4119.

30. Kelley LC, Weed SA. Cortactin Is a Substrate of Activated Cdc42-Associated Kinase 1 (ACK1) during Ligand-induced Epidermal Growth Factor Receptor Downregulation. PLoS One. 2012;7:e44363.

31. Mooren OL, Kotova TI, Moore AJ, Schafer DA. Dynamin2 GTPase and cortactin remodel actin filaments. J Biol Chem. 2009;284:23995-4005.

32. Basu D, Nguyen T-TK, Montone KT, Zhang G, Wang L-P, Diehl JA, et al. Evidence for mesenchymal-like sub-populations within squamous cell carcinomas possessing chemoresistance and phenotypic plasticity. Oncogene. 2010;29:4170-82.

33. Gatesman Ammer A, Hayes KE, Martin KH, Zhang L, Spirou GA, Weed SA. Multiphoton Imaging of Tumor Cell Invasion in an Orthotopic Mouse Model of Oral Squamous Cell Carcinoma. J Vis Exp. 2011;1-7.

34. Weaver AM, Heuser JE, Karginov A V., Lee W, Parsons JT, Cooper JA. Interaction of cortactin and N-WASp with Arp2/3 complex. Curr Biol. 2002;12:1270-8.

35. Uruno T, Liu J, Zhang P, Fan Y, Egile C, Li R, et al. Activation of Arp2/3 complexmediated actin polymerization by cortactin. Nat Cell Biol. 2001;3:259-66.

36. Clark ES, Whigham AS, Yarbrough WG, Weaver AM. Cortactin is an essential regulator of matrix metalloproteinase secretion and extracellular matrix degradation in invadopodia. Cancer Res. 2007;67:4227-35.

37. Siton O, Ideses Y, Albeck S, Unger T, Bershadsky AD, Gov NS, et al. Cortactin releases the brakes in actin- based motility by enhancing WASP-VCA detachment from Arp2/3 branches. Curr Biol. Elsevier Ltd; 2011;21:2092-7.

38. Kelley LC, Ammer AG, Hayes KE, Martin KH, Machida K, Jia L, et al. Oncogenic Src requires a wild-type counterpart to regulate invadopodia maturation. J Cell Sci. 2010;123:3923-32.

39. Vadlamudi RK, Li F, Barnes CJ, Bagheri-Yarmand R, Kumar R. p41-Arc subunit of human Arp2/3 complex is a p21-activated kinase-1-interacting substrate. EMBO Rep. 2004;5:154-60.

40. Kazazian K, Go C, Wu H, Brashavitskaya O, Xu R, Dennis JW, et al. Plk4 Promotes Cancer Invasion and Metastasis through Arp2/3 Complex Regulation of the Actin Cytoskeleton. Cancer Res. 2017;77:434-47. 
41. LeClaire LL, Rana M, Baumgartner M, Barber DL. The Nck-interacting kinase NIK increases Arp2/3 complex activity by phosphorylating the Arp2 subunit. J Cell Biol. Rockefeller University Press; 2015;208:161-70.

42. Siddiqui-Jain A, Drygin D, Streiner N, Chua P, Pierre F, O'Brien SE, et al. CX4945, an orally bioavailable selective inhibitor of protein kinase CK2, inhibits prosurvival and angiogenic signaling and exhibits antitumor efficacy. Cancer Res. American Association for Cancer Research; 2010;70:10288-98.

43. Zhao M, Sano D, Pickering CR, Jasser SA, Henderson YC, Clayman GL, et al. Assembly and initial characterization of a panel of 85 genomically validated cell lines from diverse head and neck tumor sites. Clin Cancer Res. 2011;17:7248-64.

44. Kurtz KA, Hoffman HT, Bridget Zimmerman M, Robinson RA, Carver LA. Perineural and Vascular Invasion in Oral Cavity Squamous Carcinoma Increased Incidence on Re-review of Slides and by Using Immunohistochemical Enhancement. Arch Pathol Lab Med. 2005.

45. Kelley LC, Hayes KE, Ammer AG, Martin KH, Weed SA. Cortactin phosphorylated by ERK1/2 localizes to sites of dynamic actin regulation and is required for carcinoma lamellipodia persistence. PLoS One. 2010;5:e13847.

46. Oser M, Condeelis J. The cofilin activity cycle in lamellipodia and invadopodia. J Cell Biochem. 2009;108:1252-62.

47. Cai L, Holoweckyj N, Schaller MD, Bear JE. Phosphorylation of coronin 1B by protein kinase $C$ regulates interaction with Arp2/3 and cell motility. J Biol Chem. 2005;280:31913-23.

48. Xing Y, Zhang J, Lin H, Gold KA, Sturgis EM, Garden AS, et al. Relation between the level of lymph node metastasis and survival in locally advanced head and neck squamous cell carcinoma. Cancer. 2016;122:534-45.

49. Fagan JJ, Collins B, Barnes L, D'Amico F, Myers EN, Johnson JT. Perineural Invasion in Squamous Cell Carcinoma of the Head and Neck. Arch Otolaryngol Neck Surg. American Medical Association; 1998;124:637. 
Figure 1. Cortactin T24 is required for binding and activation of Arp2/3 complex.

A. Diagram representing cortactin functional domains. NTA, N-terminal acidic domain; R1-R6, repeats regions with F-actin binding site indicated; Helix, alpha helical domain, PRR, proline rich region; SH3, Src homology 3 domain. NTA domain with position of S11 and T24 in context of the DDW region is shown. B. Immunoprecipitation analysis of Arp2/3 binding to cortactin NTA mutants. HEK 293T/17 cells transfected with FLAG-empty vector (EV), FLAG-wild-type cortactin (WT) or the indicated FLAG-cortactin mutants. Immune complexes were Western blotted with antibodies against cortactin (top) and Arp3 (bottom). 1:10 diluted total cell lysates were Western blotted as indicated. C. Coomassie

blue staining of the indicated purified recombinant human cortactin proteins. D. Effect of cortactin T24A on Arp2/3 complex activation. Fluorometric evaluation of actin polymerization over time with the indicated cortactin mutants incubated with Arp2/3 complex and pyrene-labeled actin. Polymerization curves: WT cortactin (blue); T24A cortactin (dark green) and $\triangle \mathrm{DDW}$ cortactin (purple). N-WASp VCA domain (black) was used as a positive control; negative controls include Arp2/3 complex plus actin (pale green) and actin alone (red). Polymerization curves are representative from three independent experiments.

Figure 2. T24 is required for cortactin-mediated invadopodia formation and ECM degradation in HNSCC cells.

A. UMSCC1 cells with stable shRNA scramble control (Ctl) or anti-cortactin shRNA combined with siRNA knockdown $(K D)$ were transduced with murine FLAG-WT, $-\triangle D D W$, -T24A and -T24D cortactin lentiviruses. Cells were plated on Oregon Green (OG)-488 gelatin coated coverslips for 12 hours, fixed, and labeled with anti-FLAG and rhodamine 
phalloidin (Actin). Gelatin panels are pseudo-colored white; degradation is evident as black areas indicating loss of fluorescence. Scale bar represents $20 \mu \mathrm{m}$. Insets in WT Cttn panel display invadopodia precursors (top right, yellow arrows) and active invadopodia (bottom left, yellow arrowheads). Inset scale bar represents $1 \mu \mathrm{m}$. B. Quantification of gelatin matrix degradation for control (Ctl), cortactin KD and FLAGcortactin expressing UMSCC1 cells. C. Quantification of invadopodia precursors (left) and active invadopodia (right) numbers from the lines assayed in (B). Data represents the mean + S.E.M. of $n \geq 100$ cells for each line analyzed from at least three independent experiments. All gelatin degradation conditions were normalized to CtI UMSCC1 cells. n.s., not significant; ${ }^{*}, \mathrm{P}<0.05$, Welch's or Student's $t$ test vs. Ctl $(B \& C), \dagger, P<0.05$, Student's $t$ test vs. wild type cortactin rescue (WT) (C). D. Total cell lysates from (A) evaluated for endogenous and FLAG-cortactin expression by immunoblotting with antibodies against cortactin (top) and $\beta$-actin (bottom).

Figure 3. CK2 $\alpha$ phosphorylation of cortactin T24 inhibits Arp2/3 complex binding and activation.

A. Cortactin T24 is a CK2 $\alpha$ phosphorylation site. Autoradiogram of active CK2 $\alpha$ incubated with the increasing amounts $(0,0.25,0.5$, and $1 \mu \mathrm{g})$ of GST-WT-NTA or GST-T24A-NTA cortactin fusion proteins. GST $(1 \mu \mathrm{g})$ and the GST-VCA domain of N-WASp $(0.25 \mu \mathrm{g})$ were used as respective negative and positive phosphorylation controls. Positions of autophosphorylated CK2 $\alpha$, GST-VCA and cortactin NTA proteins are indicated on the left; autoradiogram is representative of three independent experiments. B. CK2 $\alpha$ phosphorylation at cortactin T24 ablates binding to Arp2/3 complex. Purified recombinant human WT and T24A cortactin proteins $(2.5 \mu \mathrm{g})$ were bound with an anti-cortactin 87 
antibody to protein $\mathrm{G}$ beads. Immune complexes were preincubated with or without $75 \mathrm{ng}$ active CK2 $\alpha$, washed and incubated with $50 \mathrm{ng}$ purified Arp2/3 complex. Coimmunoprecipitated complexes were Western blotted for cortactin (top) and Arp3 (bottom). 4F11-bound protein $\mathrm{G}$ beads were used as a negative control for non-specific binding (Beads). Arp2/3 complex (5 ng) was used as positive control for Arp3 immunoblotting. Blot is representative of two independent experiments. C. Cortactin phosphorylation by CK2 $\alpha$ inhibits cortactin-mediated Arp2/3 actin polymerization. WT human cortactin or GST-VCA proteins were preincubated with the indicated amounts of active CK2 $\alpha$ and evaluated for effects on Arp2/3 activity. Polymerization curves are representative from three independent experiments. D. Phosphorylation of T24 is responsible for the inhibitory effect of $\mathrm{CK} 2 \alpha$ on cortactin-mediated Arp2/3 activation. Human WT and T24A cortactin proteins were preincubated with or without $30 \mathrm{ng}$ active CK2 $\alpha$ and evaluated for effects on Arp2/3-mediated actin assembly. Polymerization curves are representative from three independent experiments.

\section{Figure 4. CK2 $\alpha$ is required for optimal HNSCC invadopodia function.}

A. Evaluation of $\mathrm{CK} 2 \alpha$ expression in stable scramble control (Ctl) and CK2 $\alpha$ shRNA HNSCC cells. Cells were lysed and Western blotted with antibodies against CK2 $\alpha$ (top) and $\beta$-actin (bottom). B. Representative confocal images of OSC19 and UMSCC1 cells with Ctl shRNA and with each anti-CK2 $\alpha$ shRNA (shCK2 $\# 1$ and \#2). Cells were plated on OG-488 gelatin coverslips for $12 \mathrm{~h}$ then labeled with an anti-cortactin antibody and rhodamine phalloidin (Actin). Gelatin is pseudo-colored white. Scale bar represents 20 $\mu \mathrm{m}$. C. CK2 $\alpha$ knockdown decreases invadopodia-mediated ECM degradation. Quantification of matrix degradation area per cell area for $\mathrm{Ctl}$ and each anti-CK2 $\alpha$ shRNA 
in the indicated cell lines. Degradation data was normalized to Ctl condition for each cell line. D. CK2 $\alpha$ knockdown decreases invadopodia numbers. Amount of invadopodia precursors (left) and active invadopodia (right) per cell is shown for control and shCK2a OSC19 and UMSCC1 cells. Data in C and D represents the mean + S.E.M. of $n \geq 100$ cells analyzed from at least three independent experiments. n.s., not significant; *, $\mathrm{P}<$ 0.05, Welch's $t$ test vs Ctl.

Figure 5. Silmitasertib-mediated CK2 inhibition reduces invadopodia function in established HNSCC cell lines.

A. Representative confocal images of MDA1586, OSC19, and UMSCC1 cells plated on OG-488 gelatin coverslips for 1 hour before treatment with vehicle (DMSO) or $10 \mu \mathrm{M}$ Silmitasertib for 24 hours (MDA1586) or 12 hours (OSC19 \& UMSCC1) (optimal ECM degradation times for each line). Cells were fixed and labeled with an anti-cortactin antibody and rhodamine phalloidin (Actin). Gelatin is pseudo-colored white. Scale bar represents $20 \mu \mathrm{m}$. B. CK2 $\alpha$ inhibition decreases invadopodia-mediated ECM degradation. Quantification of gelatin matrix degradation area per cell area in HNSCC cell lines treated with the indicated Silmitasertib concentrations. C. CK2 $\alpha$ inhibition decreases invadopodia numbers. Amount of invadopodia precursors (left) and active invadopodia (right) per cell is shown for Silmitasertib treated HNSCC cell lines. Degradation data was normalized to DMSO-treated (0) cells for each cell line. Data in B and C represents the mean + S.E.M. of $n \geq 100$ cells analyzed from at least three independent experiments. n.s., not significant; *, P $<0.05$, Welch's or Student's $t$ test vs DMSO for each cell line. D. Quantitation of gelatin matrix degradation area per cell area for UMSCC1 cells expressing FLAG-WT, T24A or T24D cortactin treated with DMSO or $1 \mu \mathrm{M}$ Silmitasertib. Data 
represents the mean + S.E.M of $n \geq 75$ cells analyzed from at least three independent experiments. n.s., not significant; *, $\mathrm{P}<0.05$, Welch's or Student's $t$ test vs DMSO for each condition. E. Representative confocal images of UMSCC1 cells expressing FLAGWT, T24A, or T24D cortactin plated on OG-488 gelatin coverslips for 1 hour before treatment with vehicle (DMSO) or $1 \mu \mathrm{M}$ Silmitasertib for 12 hours. Cells were fixed and labeled with an anti-FLAG antibody and rhodamine phalloidin (Actin). Gelatin is pseudocolored white. Scale bar represents $20 \mu \mathrm{m}$.

\section{Figure 6. Silmitasertib inhibits invadopodia function in HNSCC PDX cells.}

A. Establishment of HNSCC patient-derived xenograft (PDX) tumors. Hematoxylin and eosin stained patient tumor tissue and PDXs. Patient tumors were from the alveolar ridge (AR). Scale bar represents $200 \mu \mathrm{m}$. B. Invadopodia formation in HNSCC PDX cells. Representative confocal images of WVUSCC-AR2 (AR2) and WVUSCC-AR5 (AR5) PDX cell lines. Cells were plated on OG-488 gelatin coverslips for 24 hours and labeled with an anti-cortactin antibody and rhodamine phalloidin (Actin). Gelatin is pseudo-colored white. Scale bar represents $20 \mu \mathrm{m}$. 10X representative phase contrast images (Phase) of each line are shown on the right. Scale bar represents $100 \mu \mathrm{m}$. C. Confocal images of WVUSCC-AR2 and WVUSCC-AR5 cells plated on OG-488 gelatin coverslips, allowed to attach for 1 hour, then treated with vehicle (DMSO) or $10 \mu \mathrm{M}$ Silmitasertib for 24 hours. Cells were labeled with an anti-cortactin antibody and rhodamine phalloidin (Actin). Gelatin is pseudo-colored white. Scale bar represents $20 \mu \mathrm{m}$. D. CK2 $\alpha$ inhibition decreases invadopodia-mediated ECM degradation in PDX derived cell lines. Quantification of matrix degradation area per cell area for WVUSCC-AR2 and WVUSCCAR5 PDX cell lines treated with the indicated Silmitasertib concentrations. Degradation 
data was normalized to DMSO-treated cells for each cell line. E. CK2 $\alpha$ inhibition decreases invadopodia numbers in PDX derived cell lines. Determination of amount of invadopodia precursors (left) and active invadopodia (right) in WVUSCC-AR2 and WVUSCC-AR5 cells. Data represents the mean + S.E.M. of $n \geq 100$ cells analyzed from at least three independent experiments. ${ }^{*}, \mathrm{P}<0.05$, Welch's $t$ test vs DMSO for each cell line.

Figure 7. Silmitasertib impairs collective invasion in HNSCC.

A. Representative phase contrast microscopy images of OSC19, UMSCC1 and WVUSCC-AR5 tumor cell spheroids embedded in collagen I $(0 \mathrm{~h})$ then incubated in complete media containing $10 \mu \mathrm{M}$ Silmitasertib or vehicle (DMSO) for $48 \mathrm{~h}$. Red circles indicate the maximum radial distance at indicated time points. Scale bar represents 100 $\mu \mathrm{m}$. B. Quantitation of maximal collective invasive distance of each cell line at $48 \mathrm{~h}$ with vehicle $(0)$ or Silmitasertib $(10 \mu M)$. Data represents the mean + S.E.M. of $n \geq 10$ spheroids per cell line and condition analyzed from at least three independent experiments. ${ }^{*}, \mathrm{P}<0.05$, Welch's or Student's $t$ test vs DMSO. C. Effect of Silmitasertib on HNSCC orthotopic tumor growth. Bioluminescent monitoring of UMSCC1 cells orthotopically injected into tongues of NSG mice. Tumors were allowed to establish for one week prior to administration of Silmitasertib or equal volume vehicle (DMSO). Data represent means +/- S.E.M. from two independent experiments. DMSO, $\mathrm{n}=9$ mice; Silmitasertib, $n=7$ mice. n.s., not significant; Student's $t$ test vs DMSO. D. Silmitasertib inhibits orthotopic HNSCC invasion. Representative hematoxylin and eosin stained invasive front of orthotopic UMSCC1 tongue tumors from mice receiving Silmitasertib or vehicle (DMSO) for three weeks. Black lines show tumor-encompassing RIO borders 
determined by software analysis. Black box in DMSO tumor indicates the invasive protrusion shown in $\mathbf{F}$. White box in DMSO tumor denotes region containing perineural invasion (PNI) shown in I. Black arrow indicates direction of invasion toward tongue base. M, skeletal muscle; T, tumor. Scale bar represents $100 \mu \mathrm{m}$. E. Invasive distance of tumor protrusions from mice treated with Silmitasertib or vehicle (DMSO). Data represent the mean + S.E.M. of two serial sections from $\mathrm{N}=4$ tumors; $\mathrm{n}=34$ protrusions per condition. ${ }^{*}, \mathrm{P}<0.05$, Student's $t$ test vs DMSO. F. Representative image of an invasive protrusion from vehicle-treated mice shown in the black box in $\mathbf{D}$. The protrusive region was traced in black to denote the tumor protrusion from surrounding muscle-containing stroma. $\mathrm{M}$, skeletal muscle; T, tumor protrusion. Scale bar represents $100 \mu \mathrm{m}$. G. Area of invasive protrusions from mice treated with Silmitasertib or vehicle (DMSO). Data represent the mean + S.E.M. of two serial sections from $N=4$ tumors; $n=34$ protrusions per condition. ${ }^{*}, \mathrm{P}<0.05$, Welch's $t$ test vs DMSO. H. Percentage of lingual nerves displaying PNI in tumors from mice treated with Silmitasertib or vehicle (DMSO). Data represent the mean + S.E.M. of two serial sections from $N=4$ tumors; $n \geq 60$ nerves per condition. *, $P<$ 0.05 , Student's $t$ test vs DMSO. I. Representative image of PNI shown in the white box in the DMSO-treated mouse in D. N, nerve; T, tumor cells. Scale bar represents $50 \mu \mathrm{m}$. 
CK2 regulates cortactin function in tumor invasion CK2 phosphorylates cortactin T24 in the Arp2/3 binding domain

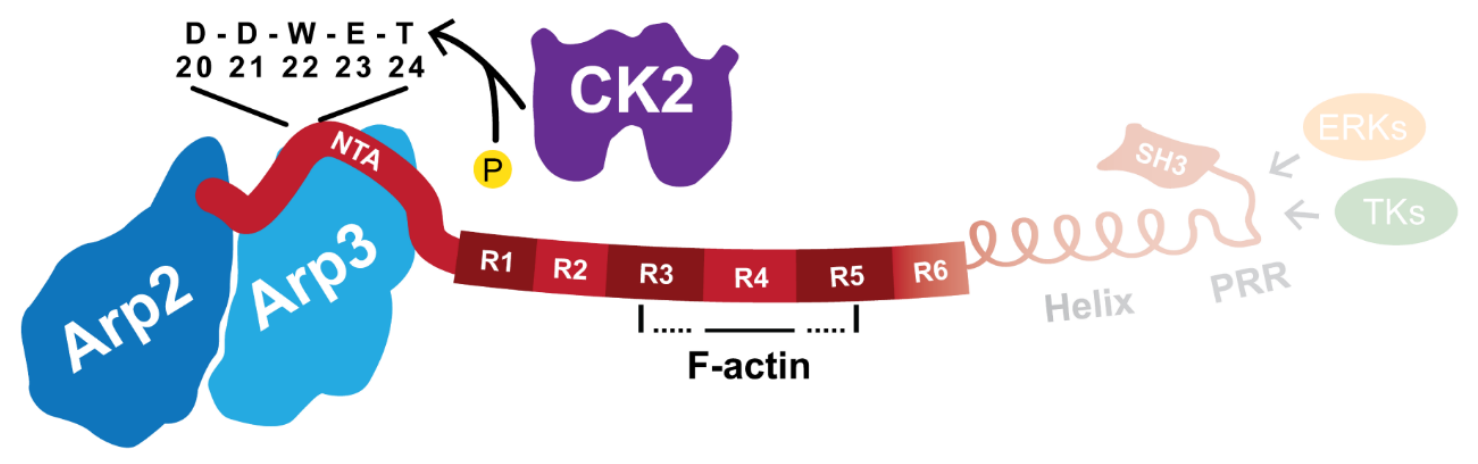

Cortactin-CK2 activity cycle in promoting tumor invasion

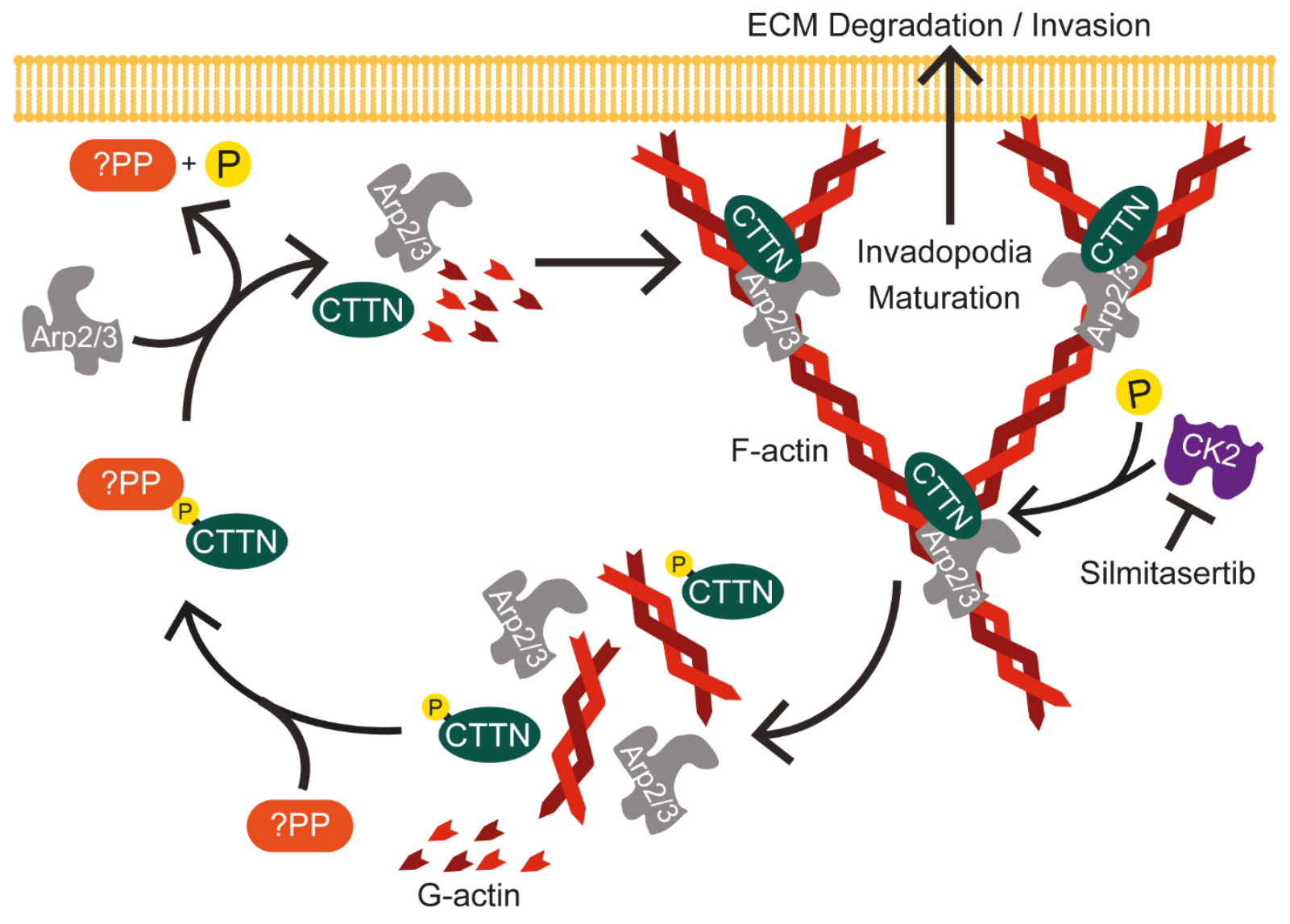

Graphical Abstract Markwell et al. 
A
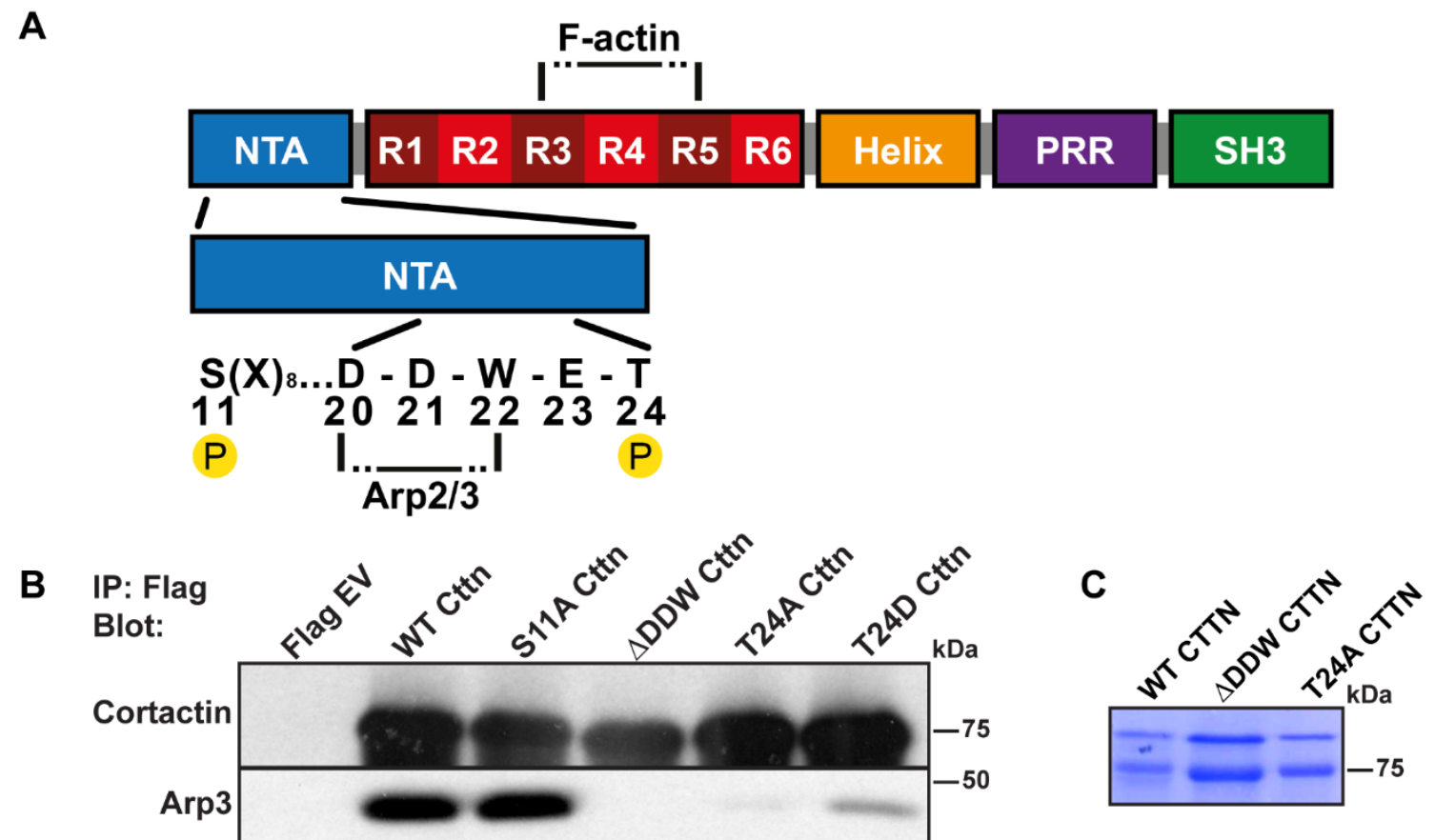

1:10 Input

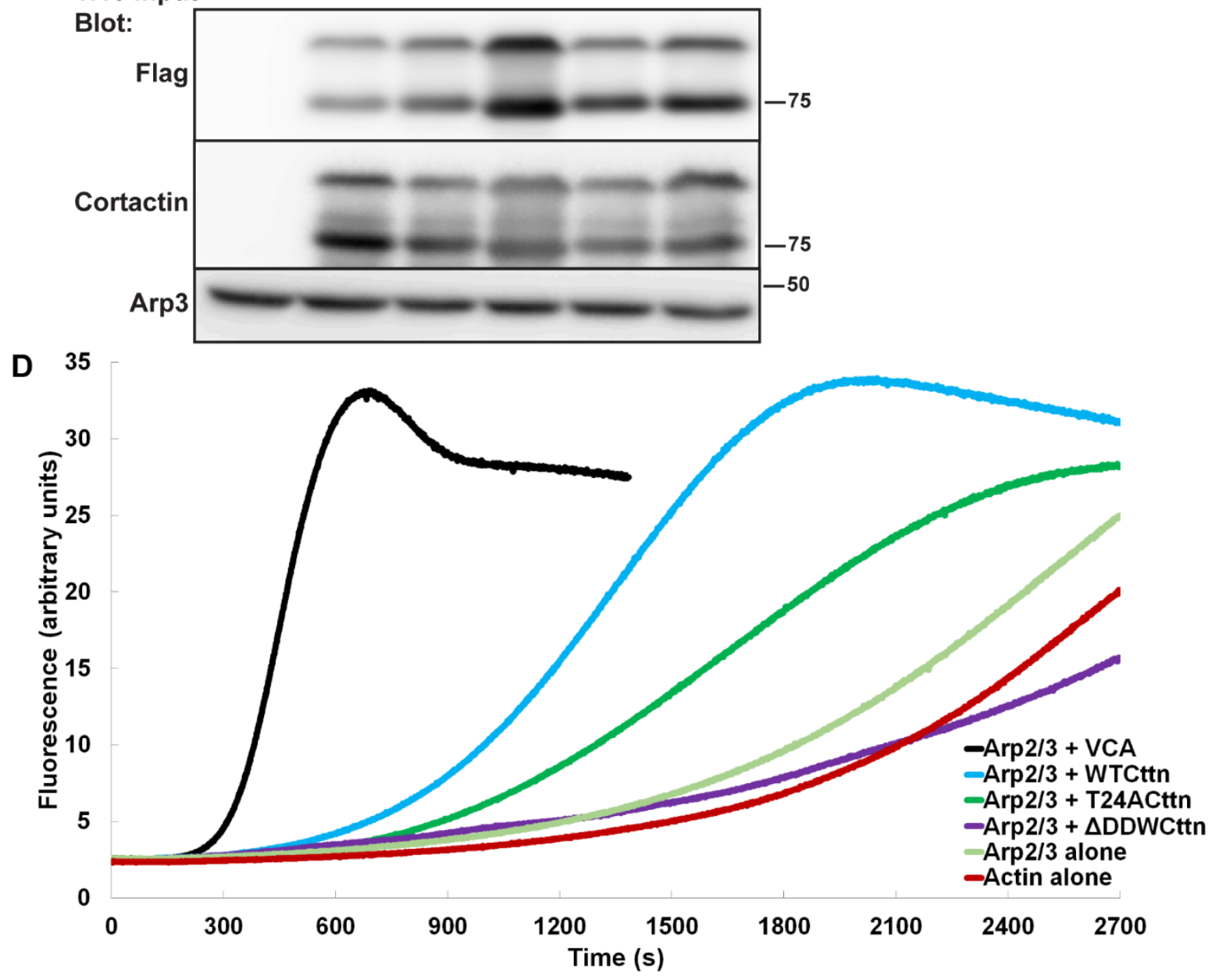

Figure 1 Markwell et al. 

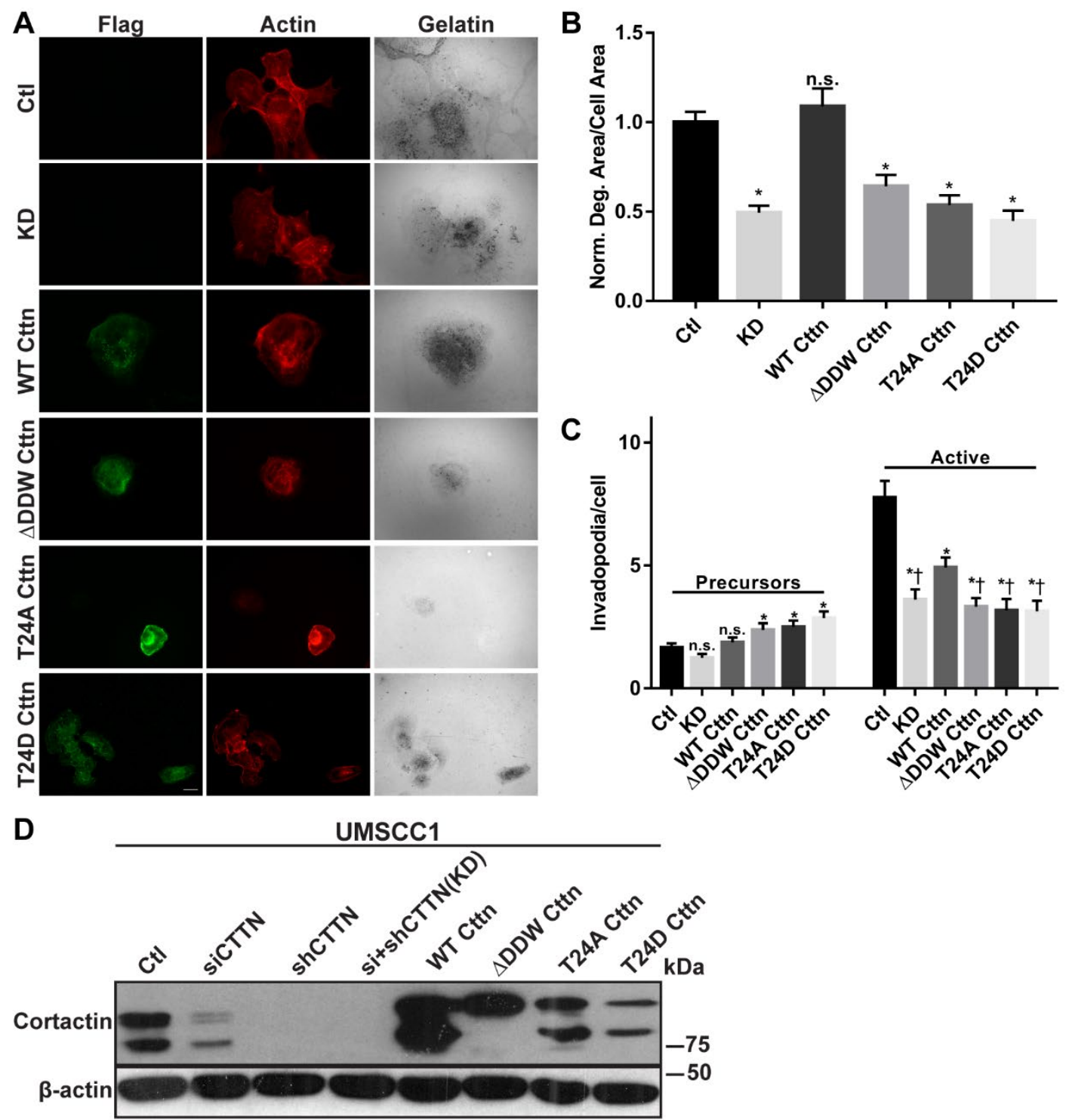

Figure 2 Markwell et al 

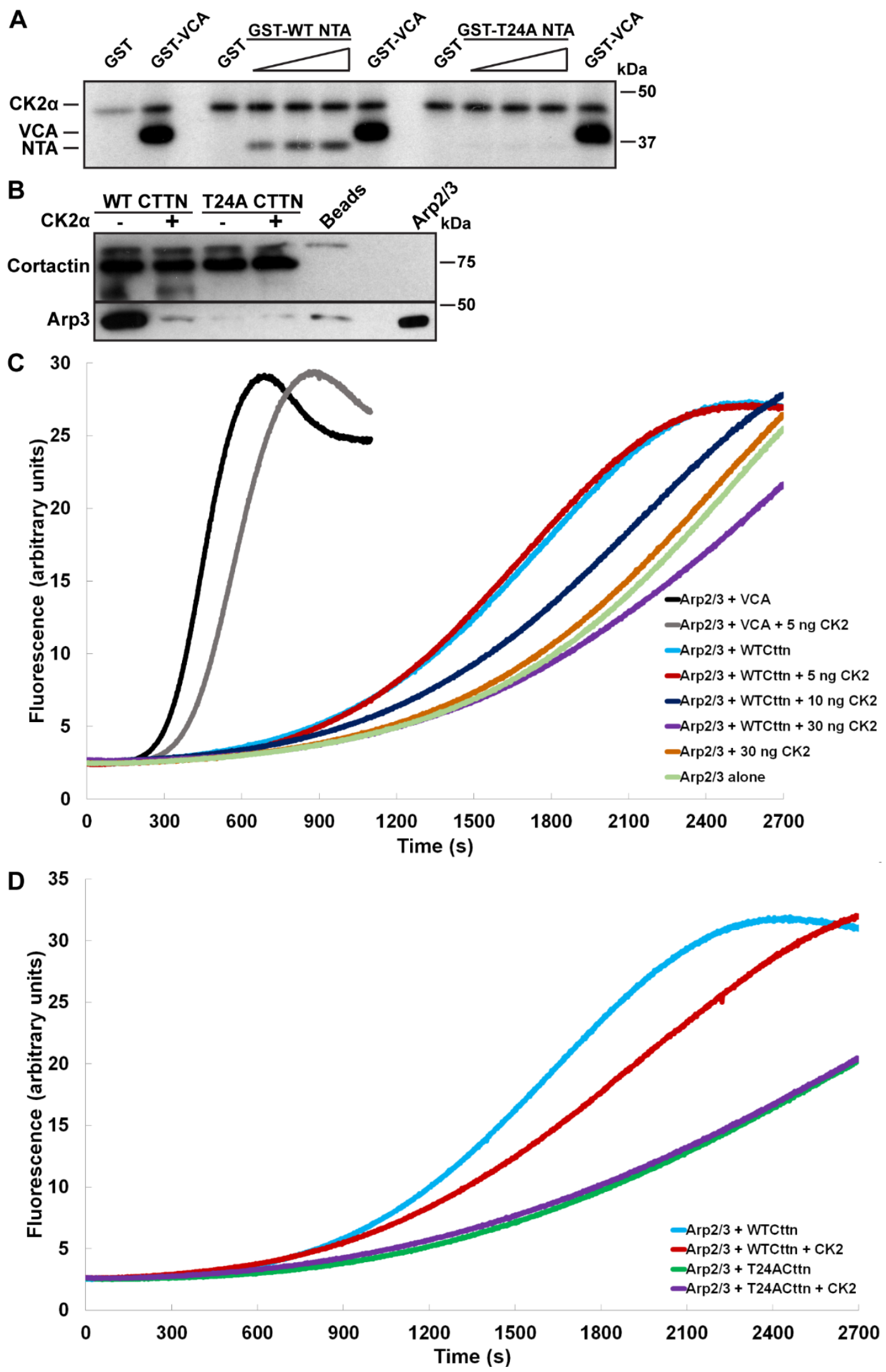

Figure 3 Markwell et al. 

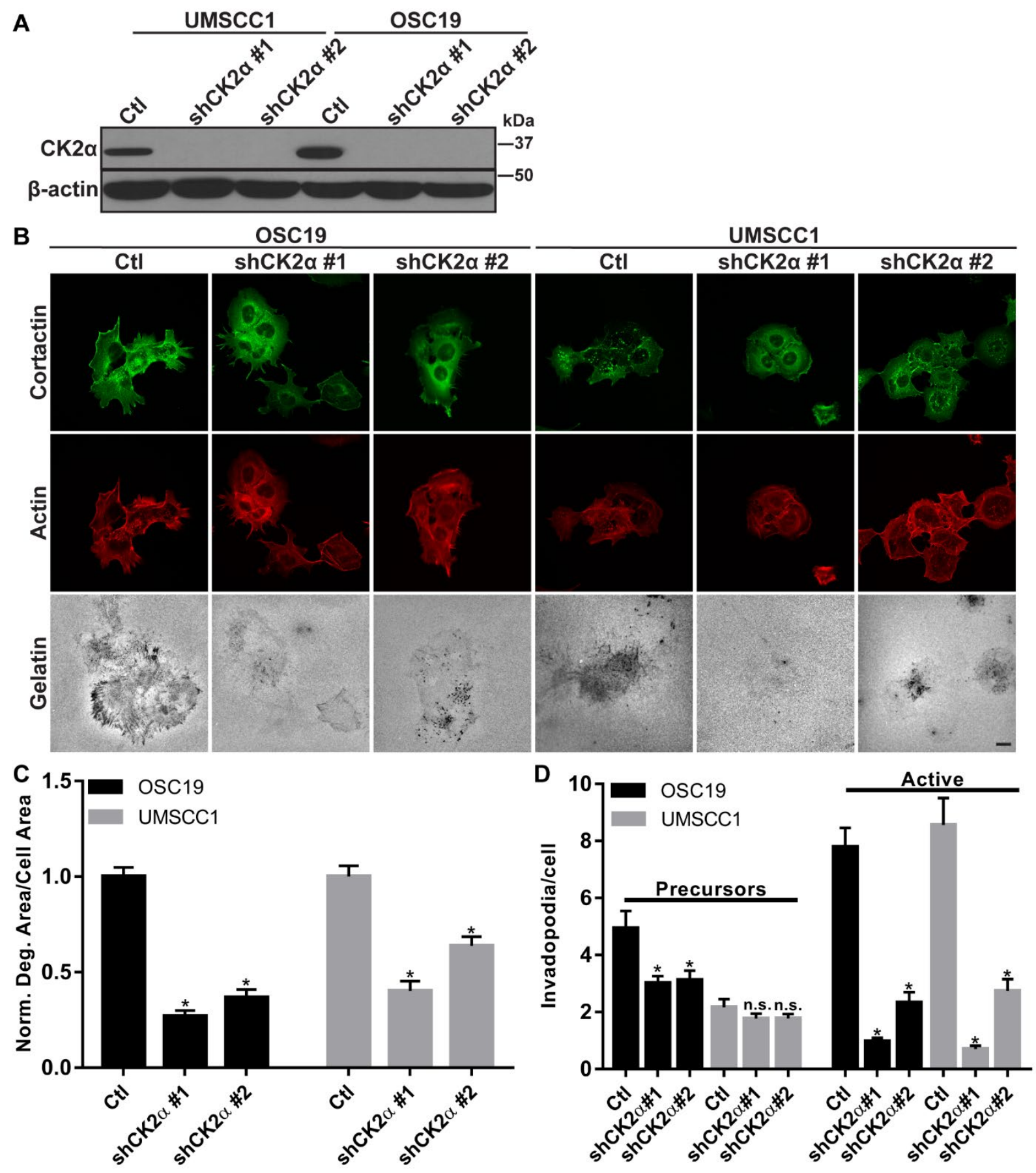

Figure 4 Markwell et al. 


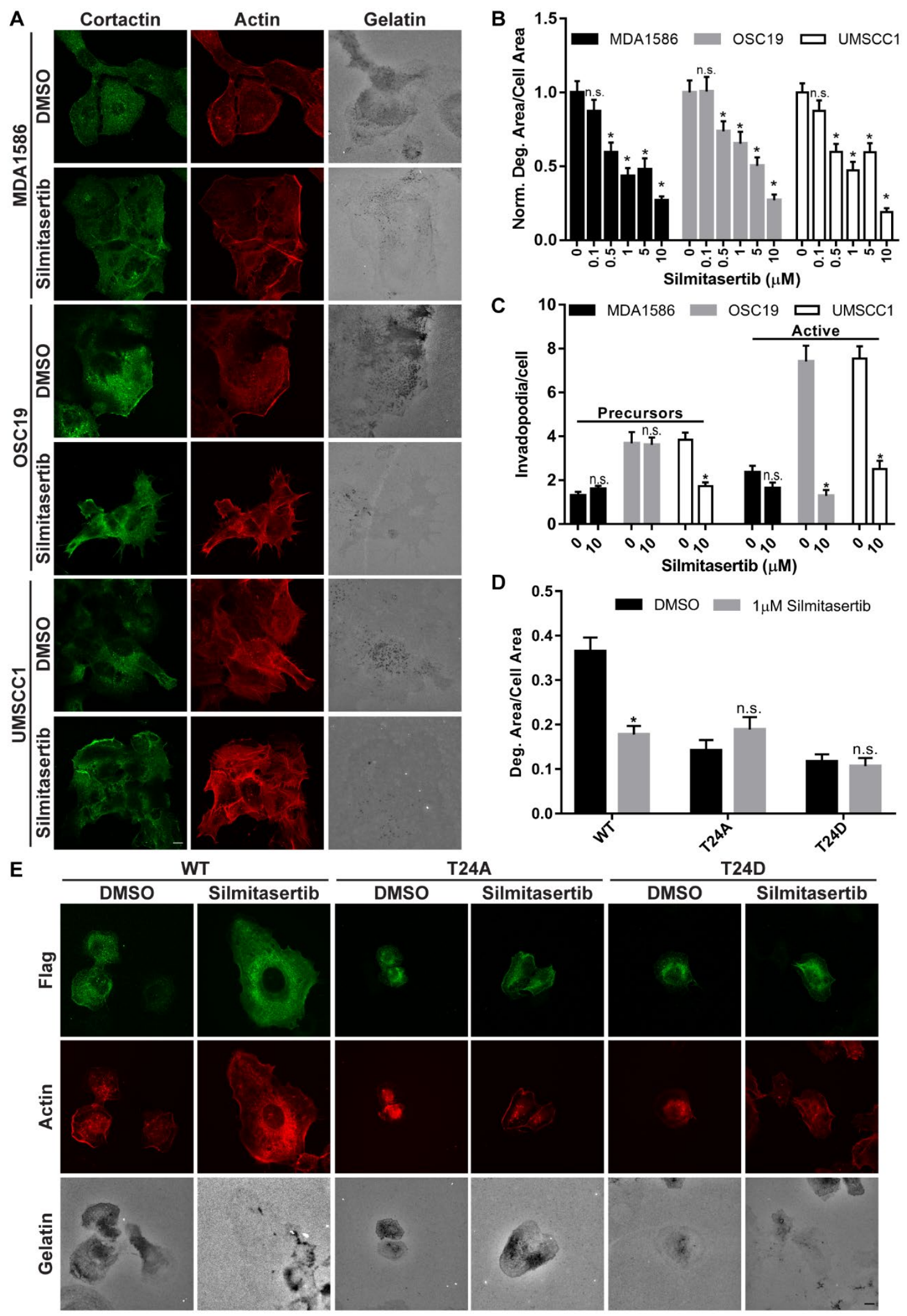

Figure 5 Markwell et al. 


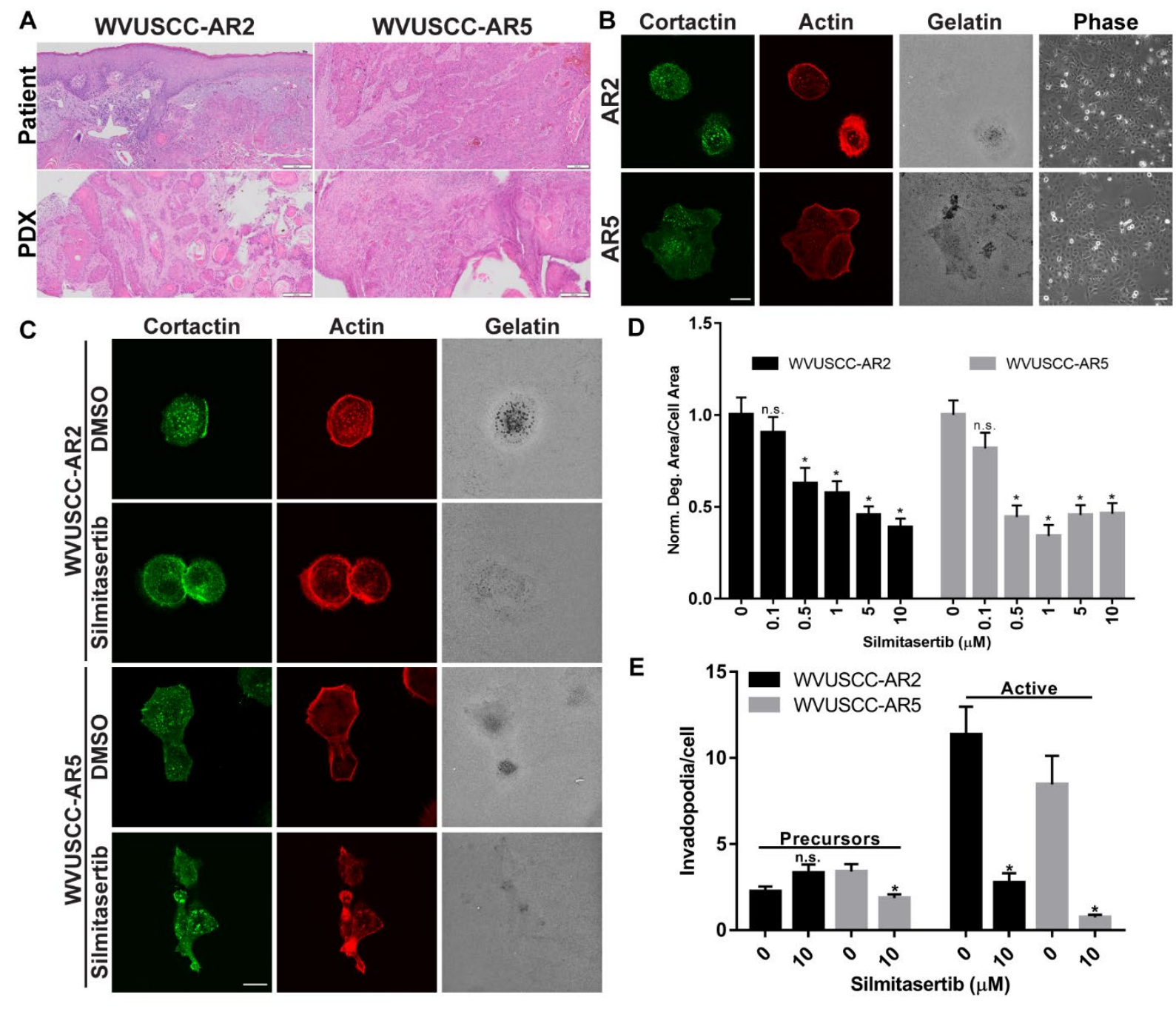

Figure 6 Markwell et al. 
A
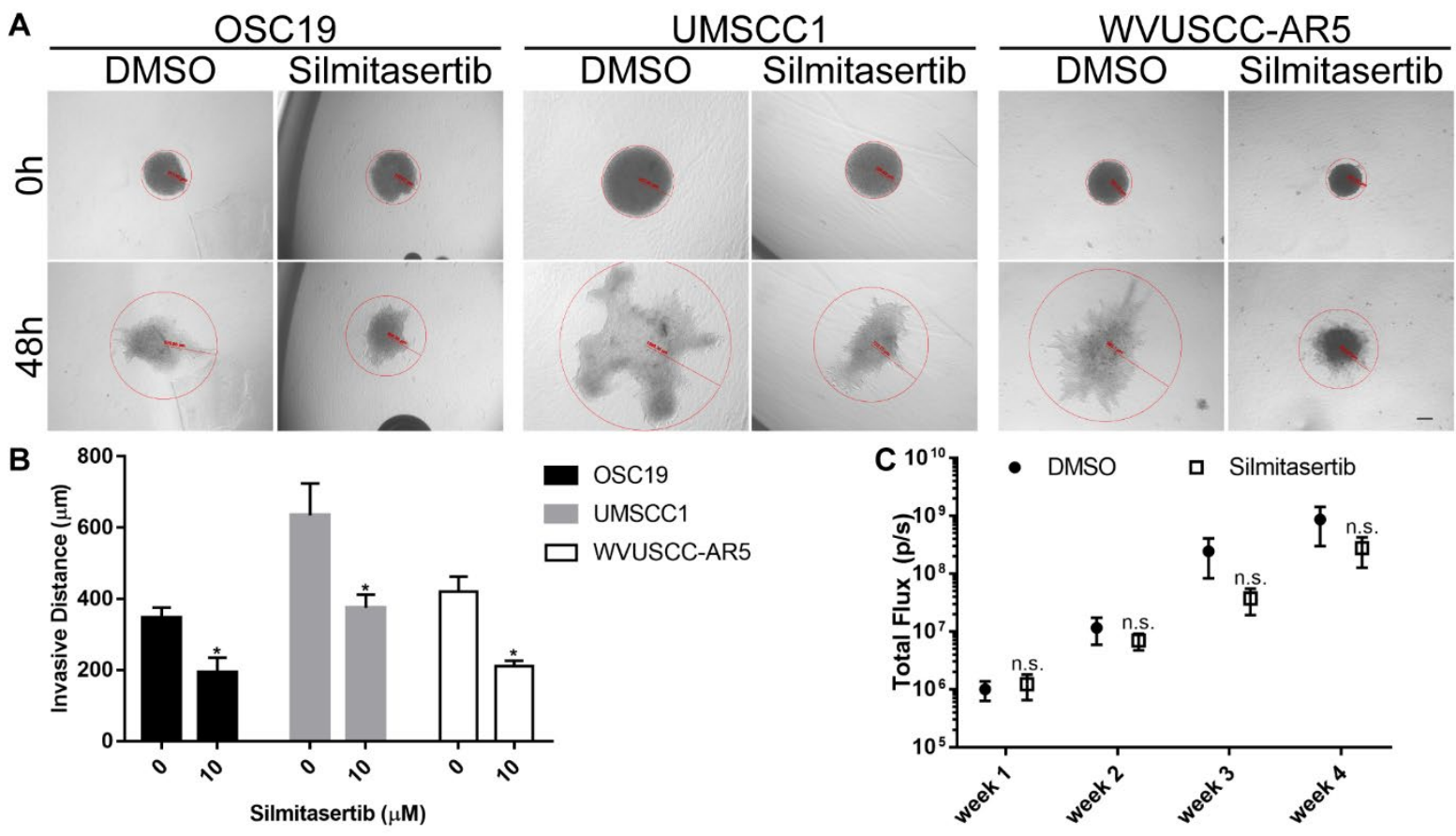

D
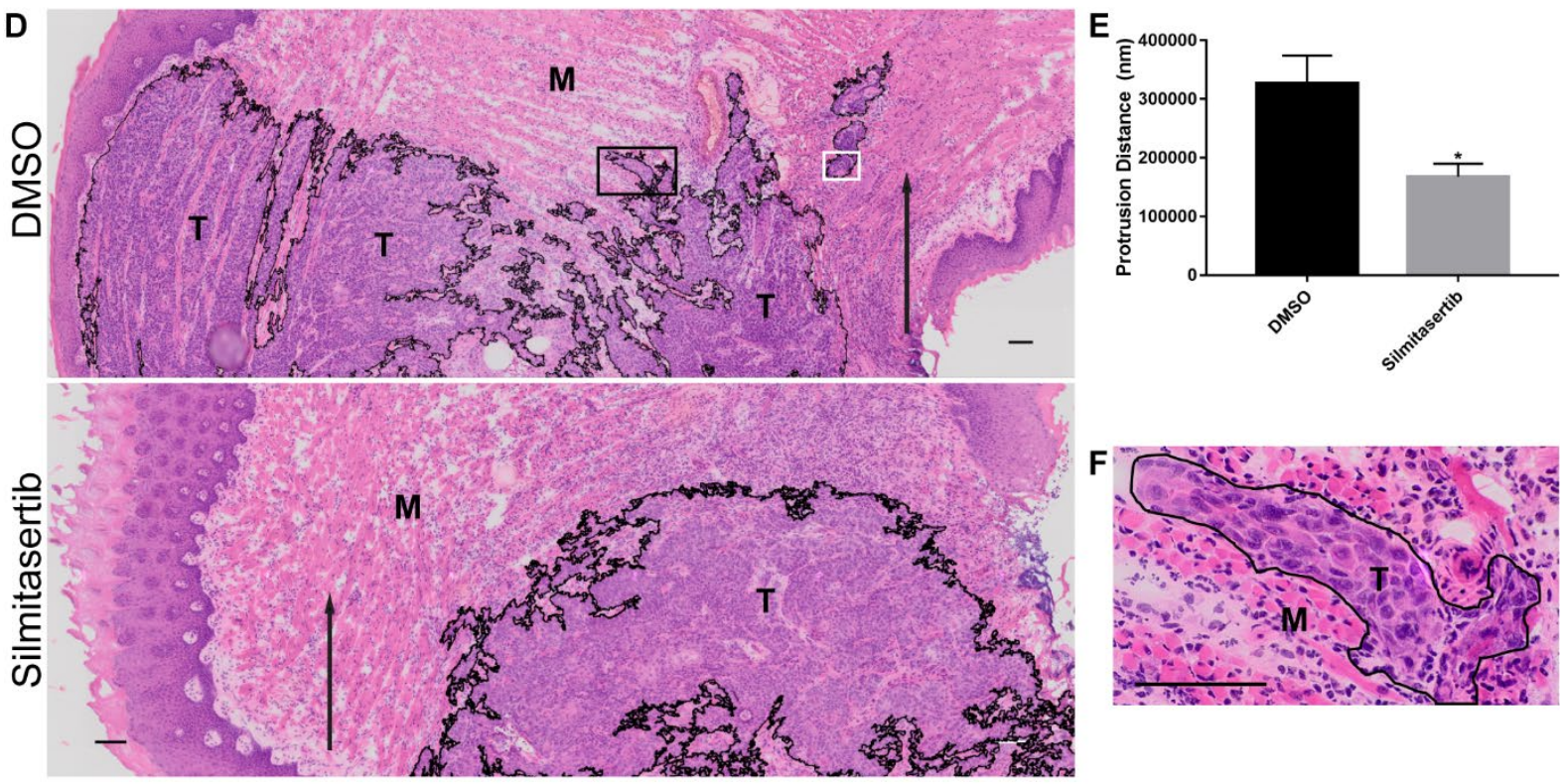

G

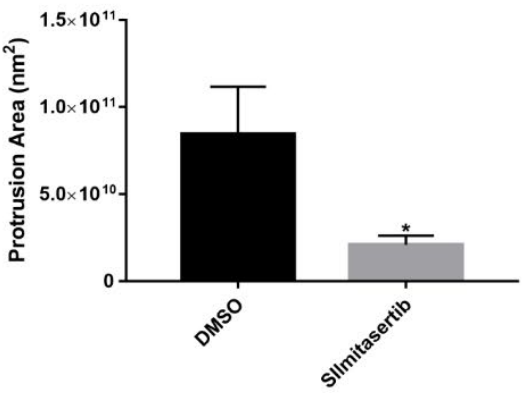

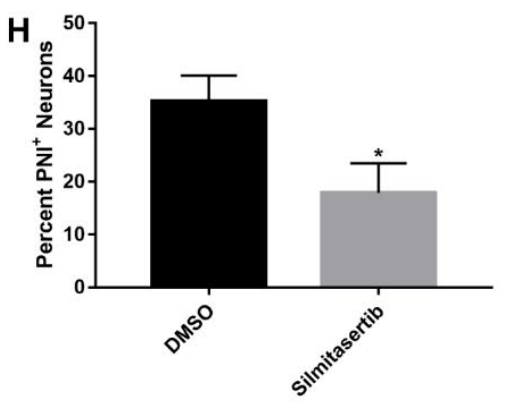

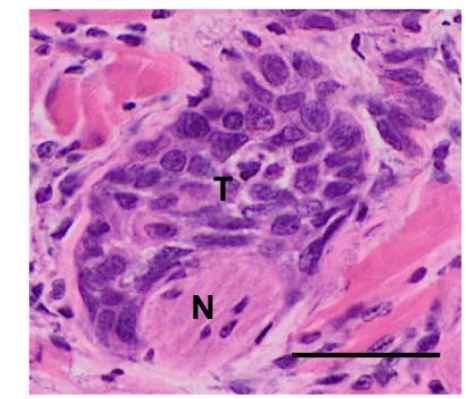

Figure 7 Markwell et al. 


\section{Supplementary Materials}

\section{Supplementary Figure S1. T24 is required for optimal OSC19 invadopodia function.}

A. Representative epifluorescent images of OSC19 cells with stable shRNA scramble control (Ctl) or anti-cortactin shRNA combined with siRNA knockdown (KD) transduced with murine FLAG-WT, $-\triangle D D W,-T 24 A$ and -T24D cortactin lentiviruses. Cells were plated on Oregon Green(OG)-488 gelatin coated coverslips for 12 hours, fixed, and labeled with anti-FLAG and rhodamine phalloidin (Actin). Gelatin panels are pseudo-colored white; degradation is evident as black areas indicating loss of fluorescence. Scale bar represents $20 \mu \mathrm{m}$. B. Total cell lysates from (A) evaluated for endogenous and FLAGcortactin expression by immunoblotting with antibodies against cortactin (top) and $\beta$-actin (bottom). C. Quantification of gelatin matrix degradation for cortactin KD and FLAGcortactin expressing OSC19 cells. Data represents the mean + S.E.M. of $n \geq 100$ cells for each line analyzed from at least three independent experiments. All conditions were normalized to control (Ctl) OSC19 cells. n.s., not significant; *, P $<0.05$, Welch's $t$ test vs. Ctl. D. Representative epifluorescent images of UMSCC1 cells stably expressing shRNA scramble control $(\mathrm{Ctl})$ or murine FLAG-WT, $-\triangle \mathrm{DDW},-\mathrm{T} 24 \mathrm{~A}$ and $-\mathrm{T} 24 \mathrm{D}$ cortactin lentiviruses plated on Oregon Green(OG)-488 gelatin coated coverslips for 12 hours, fixed, and labeled with anti-cortactin or anti-FLAG and rhodamine phalloidin (Actin), as indicated. Gelatin panels are pseudo-colored white; degradation is evident as black areas indicating loss of fluorescence. Scale bar represents $20 \mu \mathrm{m}$. Insets denote invadopodia precursors (yellow; top right corners) and active invadopodia (magenta; bottom left corners). Invadopodia precursors comprise cortactin and actin puncta lacking associated 
gelatin degradation (yellow arrowheads). Active invadopodia contain cortactin and actin with associated gelatin degradation (magenta arrowheads). Inset scale bar represents 2 $\mu \mathrm{m}$.

\section{Supplementary Figure S2. Effects of stable and transient cortactin knockdown on HNSCC invadopodia function.}

A. Representative epifluorescent images of OSC19 cells with stable scramble shRNA control (Ctl), anti-cortactin siRNA knockdown (siCTTN), anti-cortactin shRNA knockdown (shCTTN), or both shRNA and siRNA cortactin treatment (KD). Cells were plated on OG488 gelatin coverslips for 12 hours and labeled with an anti-cortactin antibody and rhodamine phalloidin (Actin). Gelatin is pseudo-colored white. Scale bar represents 20 $\mu m$. Effects of each knockdown condition on cortactin expression are found in the indicated RNAi lanes of the Western blot in Supplemental Figure S1B. B. Quantification of matrix degradation area per cell area for the indicated OSC19 cell lines. All conditions were normalized to control OSC19 gelatin degradation (Ctl). Data represents the mean + S.E.M. of $n \geq 100$ cells per condition analyzed from at least three independent experiments. Ctl and KD conditions are identical to those displayed in Supplemental Figure S1. C. Representative epifluorescent images of UMSCC1 cells with stable scramble control (Ctl), cortactin siRNA knockdown (siCTTN), cortactin shRNA knockdown (shCTTN), or both RNAi conditions (KD). Cells plated on OG-488 gelatin for 12 hours were labeled with an anti-cortactin antibody and rhodamine phalloidin (Actin). Gelatin is pseudo-colored white. Scale bar represents $20 \mu \mathrm{m}$. Cortactin expression for the indicated RNAi lanes of the Western blot are shown in Figure 2D. D. Quantification of matrix degradation area per cell area for the indicated UMSCC1 cell lines. Conditions were 102 
normalized to control (CtI). Data represents the mean + S.E.M. of $n \geq 100$ cells per condition analyzed from at least three independent experiments. Ctl and KD conditions are identical to those displayed in Figure 2.

\section{Supplementary Figure 3. Silmitasertib inhibits CK2-dependent phosphorylation.}

HNSCC cells incubated with 0,1 or $10 \mu \mathrm{M}$ Silmitasertib for 24 hours (MDA1586) or 12 hours (OSC19 and UMSCC1) were lysed and evaluated for CK2 $\alpha$ inhibition by Western blotting with antibodies against phospho-serine 473 AKT (pS473; top), pan AKT (middle), and $\beta$-actin (bottom).

\section{Supplementary Figure 4. Silmitasertib treatment does not impact collective invasive groups at the tumor invasive front.}
A. Quantitation of invasive distance and B. area of collective invasive group in tumors from mice treated with Silmitasertib or vehicle (DMSO). Data represent the mean + S.E.M. of two serial sections from $N=4$ tumors, $n \geq 54$ collective groups per condition. n.s., not significant; Welch's $t$ test vs DMSO. 


\section{Supplemental Materials and Methods}

\section{Plasmid constructs}

FLAG-tagged murine cortactin expression constructs were generated as described (1). Briefly, murine cortactin cDNAs were PCR amplified as Xbal-Sall fragments and subcloned into Xbal-Sall digested pLenti CMV GFP Hygro (\#17446, Addgene). GSTtagged full length and NTA human cortactin and N-WASp VCA expression constructs were generated as before (2). Full length human cortactin cDNAs were PCR amplified as Clal-Bglll fragments and subcloned into Clal-Bglll digested pGST-parallel2. Purification of recombinant proteins were performed as before (3), with GST tags removed by AcTEV protease (\#12575015, Invitrogen). The pLU-Luc2 lentiviral vector was obtained from Elena Pugacheva (West Virginia University, Morgantown, WV).

\section{Tissue sectioning, staining and microscopy}

HNSCC patient and PDX tissue samples were fixed in 10\% neutral-buffered formalin and paraffin embedded (FFPE). Extracted orthotopic tongue tumors were frozen in O.C.T. media (4583, Scigen) using a HM 525 cryostat (Thermo Scientific). For patient tissue blocks, five micron sections were cut and dried onto charged glass slides at $60-65^{\circ} \mathrm{C}$ for 30 minutes. Slides were washed three times in xylene (\#8400-1, Statlabs) for two minutes each, rinsed in $100 \%, 95 \%$, and $80 \%$ ethanol (\#7100-1, Statlabs) sequentially for 20 seconds each, rinsed with distilled water twice for 10 seconds each, then incubated in hematoxylin (\#7211, Richard Allan Scientific) for 90 seconds. Slides were washed twice in distilled water for 20 seconds each, then incubated with bluing solution $(0.3 \%$ ammonium hydroxide, A669-212, Fisher Scientific) for 10 seconds and rinsed twice in 
distilled water for 20 seconds. Slides were washed with acid alcohol solution $(0.1 \%$ hydrochloric acid in 70\% ethanol, A144-212, Fisher Scientific), twice with distilled water for 1 minute, then with $80 \%$ ethanol for 10 seconds. Slides were incubated in eosin (\#3801600, Lieca Biosystems) for 15 seconds, washed twice with 95\% ethanol, three times with $100 \%$ ethanol, then three times with xylene for 20 seconds. Slides were mounted using a Tissue-Tek SCA coverslipper (Model 4764, Sakura).

For PDX tumors, FFPE sections were incubated three times in microwave-heated xylene for 3 minutes each. PDX tumor and orthotopic tongue tumor sections were washed thrice in $100 \%$ ethanol, then once in $96 \%$ ethanol for 1 minute. Samples were washed with distilled water, incubated with hematoxylin for 30 seconds, rinsed with water for 1 minute, then with $96 \%$ ethanol. Slides were subsequently incubated with eosin for 2 minutes, washed with water for 10 seconds, then rinsed in $96 \%$ ethanol. Slides were washed with $100 \%$ ethanol, followed by three 1 minute xylene washes. Slides were dried and mounted with glass coverslips using Permount (SP15-500, Fisher). Histological images were acquired with an Olympus VS120 Virtual Slide microscope with a UPlanSApo 20X/0.75 objective using VS-ASW-S6 software (Olympus Corporation).

\section{Predictive analysis of human cortactin T24 phosphorylating kinases}

The 84 amino acid sequence containing the entire human cortactin NTA domain was evaluated for potential phosphorylating kinases targeting the T24 site using the publicly available web-based programs ScanSite 2.0 (4), Minimotif Miner (5), PhosphoMotif finder (6), NetPhosK 1.0 (7), GPS 2.0 (8), PPSP (9) and KinasePhos (10). Potential kinases 
were ranked by frequency based on the number of different programs identifying the same kinase and are displayed in Supplementary Table S1.

\section{Tumor invasive group characterization and quantitation}

Collective groups were defined as independent groups of tumor cells that were discontinuous with the main tumor mass and progressed towards the base of tongue. Invasive distance represents the difference between the farthest edge of the collective group and the nearest border of the primary tumor mass. Quantitation of these groups was carried out as described for tumor invasive protrusions in the Materials and Methods.

\section{References}

1. Kelley LC, Ammer AG, Hayes KE, Martin KH, Machida K, Jia L, et al. Oncogenic Src requires a wild-type counterpart to regulate invadopodia maturation. J Cell Sci. 2010;123:3923-32.

2. Weed SA, Karginov A V., Schafer DA, Weaver AM, Kinley AW, Cooper JA, et al. Cortactin localization to sites of actin assembly in lamellipodia requires interactions with F-actin and the Arp2/3 complex. J Cell Biol. 2000;151:29-40.

3. Evans J V, Ammer AG, Jett JE, Bolcato C a, Breaux JC, Martin KH, et al. Src binds cortactin through an $\mathrm{SH} 2$ domain cystine-mediated linkage. J Cell Sci. 2012;125:6185-97.

4. Obenauer JC, Cantley LC, Yaffe MB. Scansite 2.0: Proteome-wide prediction of cell signaling interactions using short sequence motifs. Nucleic Acids Res. 2003;31:3635-41.

5. Balla S, Thapar V, Verma S, Luong T, Faghri T, Huang C-H, et al. Minimotif Miner: a tool for investigating protein function. Nat Methods. 2006;3:175-7.

6. Amanchy R, Periaswamy B, Mathivanan S, Reddy R, Tattikota SG, Pandey A. A curated compendium of phosphorylation motifs. Nat Biotechnol. 2007;25:285-6.

7. Blom N, Sicheritz-Pontén T, Gupta R, Gammeltoft S, Brunak S. Prediction of posttranslational glycosylation and phosphorylation of proteins from the amino acid sequence. Proteomics. 2004;4:1633-49. 
8. Xue Y, Ren J, Gao X, Jin C, Wen L, Yao X. GPS 2.0, a tool to predict kinasespecific phosphorylation sites in hierarchy. Mol Cell Proteomics. 2008;7:1598608.

9. Xue Y, Li A, Wang L, Feng H, Yao X. PPSP: prediction of PK-specific phosphorylation site with Bayesian decision theory. BMC Bioinformatics. 2006;7:163.

10. Huang H-D, Lee T-Y, Tzeng S-W, Horng J-T. KinasePhos: a web tool for identifying protein kinase-specific phosphorylation sites. Nucleic Acids Res. 2005;33:W226-9. 


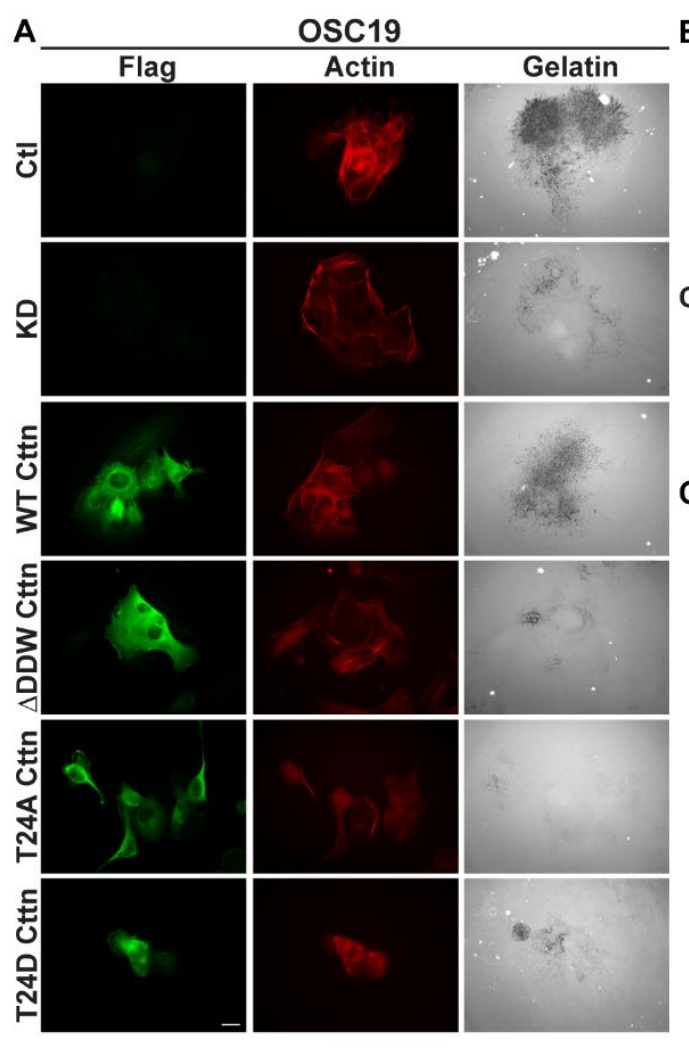

B
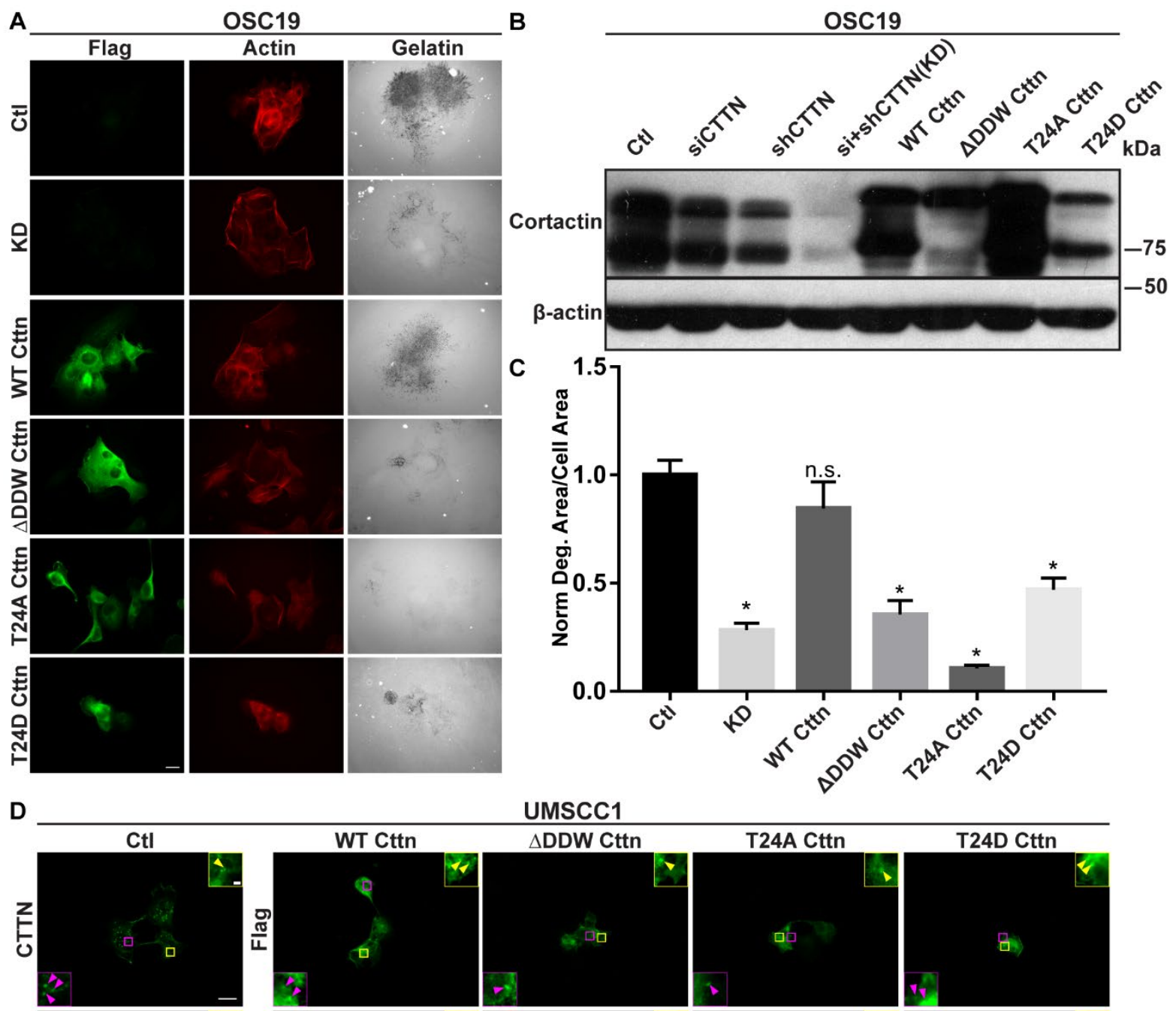

C

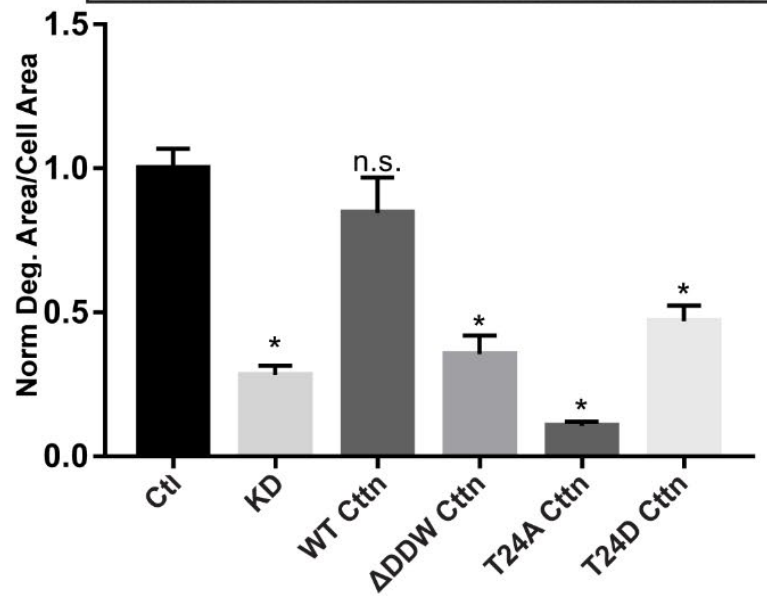

UMSCC1

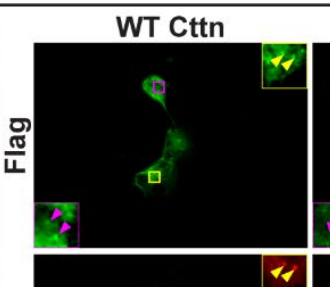

$\triangle \mathrm{DDW}$ Cttn

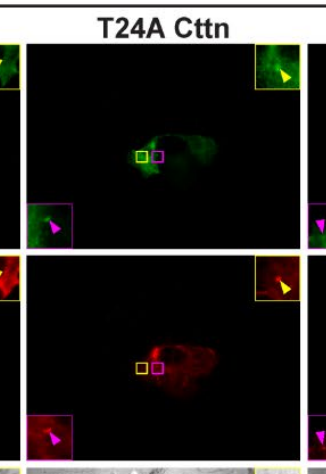

T24D Cttn
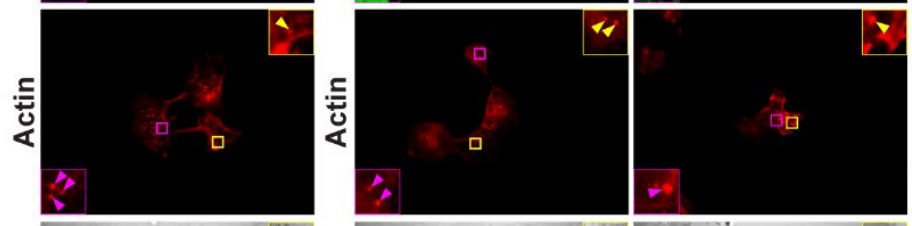

6. 5

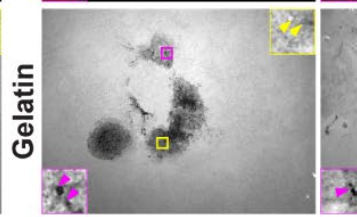

Supplementary Figure 1 Markwell et al. 
A

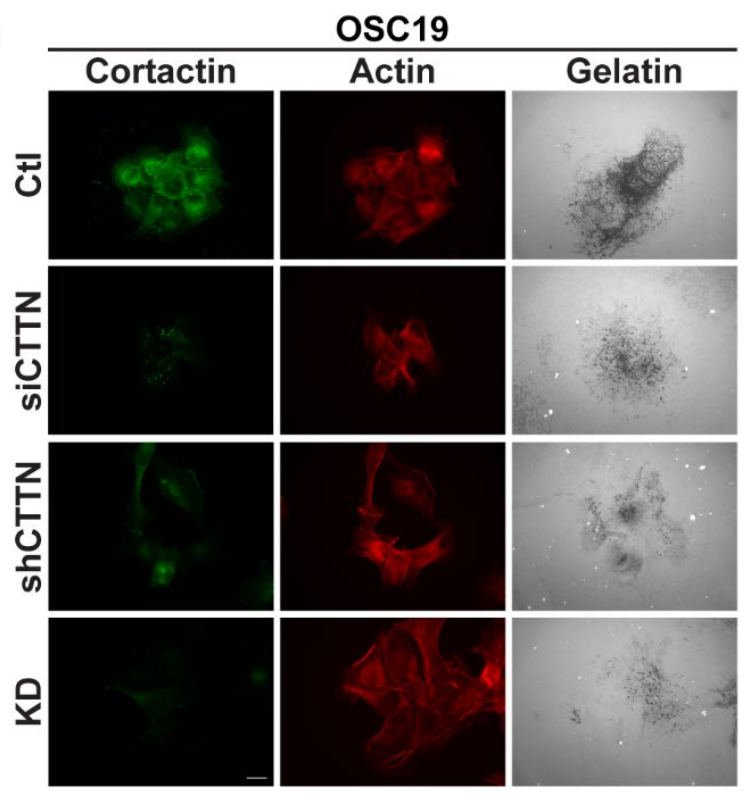

B

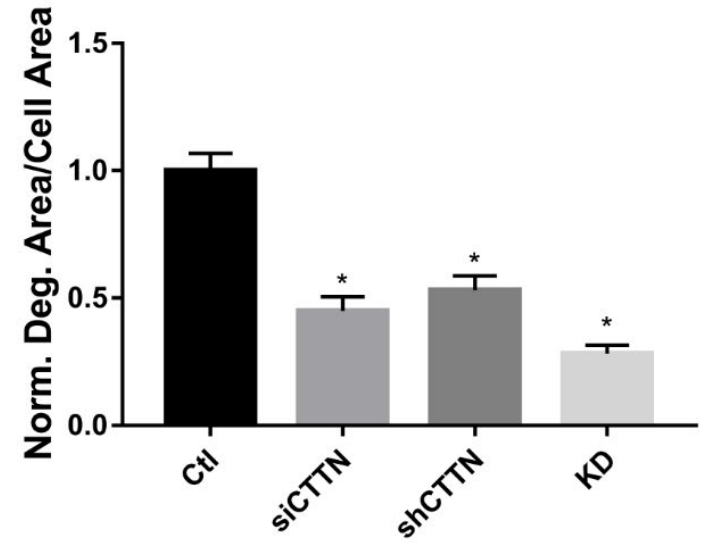

C
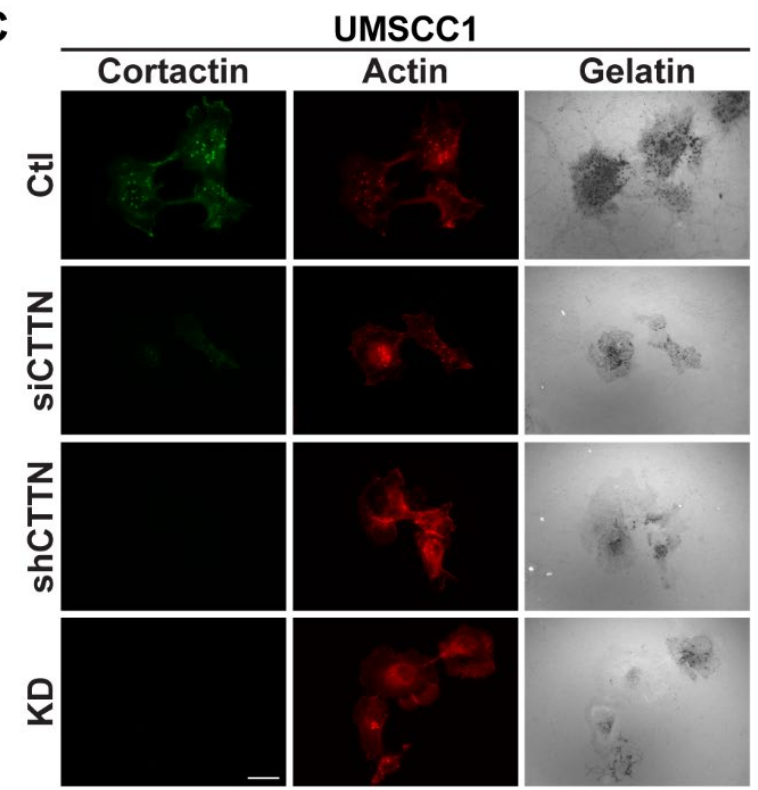

D

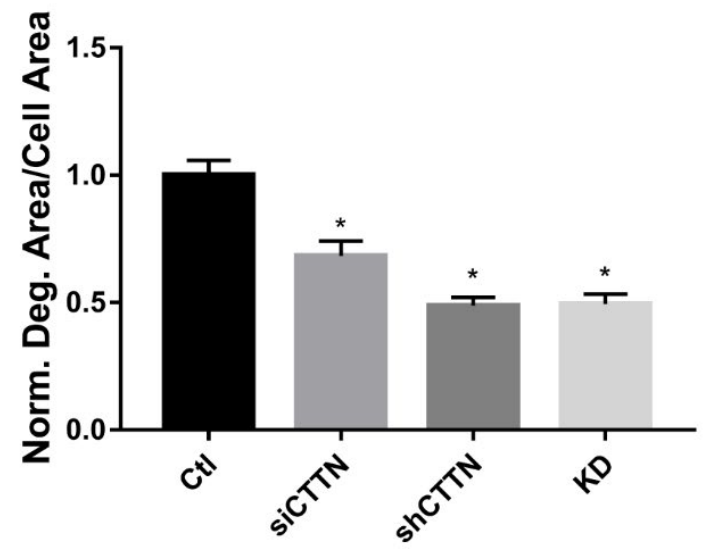

Supplementary Figure 2 Markwell et al. 


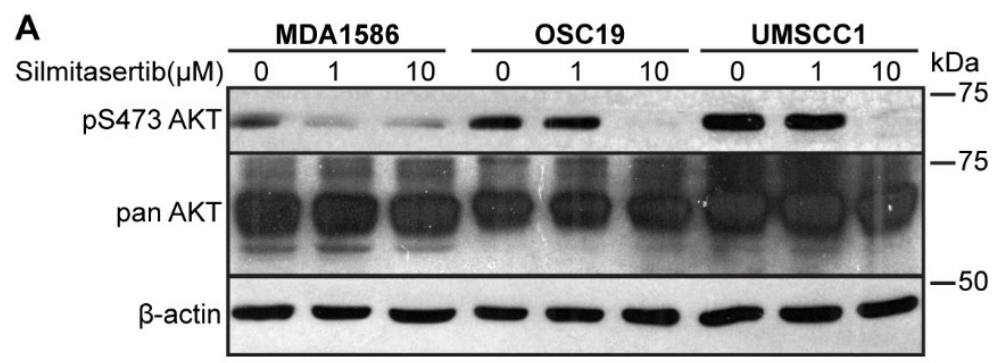

Supplementary Figure 3 Markwell et al. 

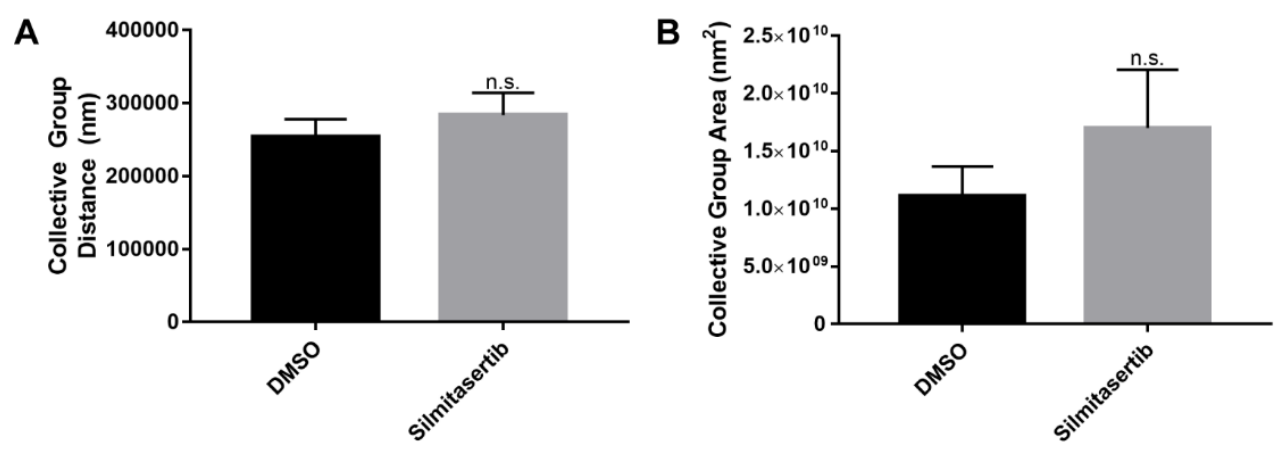

Supplementary Figure 4 Markwell et al. 
Supplemental Table S1. Predictive analysis of human cortactin T24 phosphorylating kinases

\begin{tabular}{|c|c|c|c|c|c|c|c|}
\hline Kinase & ScanSite & $\begin{array}{l}\text { Minimotif } \\
\text { Miner }\end{array}$ & $\begin{array}{l}\text { Phospho } \\
\text { Motif } \\
\text { finder }\end{array}$ & NetPhosK & GPS & PPSP & KinasePhos \\
\hline CK2 & $x$ & $x$ & $X$ & $X$ & $X$ & $X$ & \\
\hline CK1 & & & $X$ & & & $x$ & \\
\hline GRK & & & & & $X$ & $x$ & $x$ \\
\hline DNA-PK & & & & & & $X$ & \\
\hline PKB & & & & & & & $X$ \\
\hline $\begin{array}{l}\text { Protein } \\
\text { MWKAS, }\end{array}$ & IVSIAC & AGA & ETDF & IS & מיח & QQ & Sequence: \\
\hline
\end{tabular}




\section{Study 2: Establishment of an Appalachian Head and Neck}

\section{Squamous Cell Carcinoma Patient-Derived Xenograft Cohort}

Steven M. Markwell ${ }^{1}$, Jessica A. Allen¹, River A. Hames ${ }^{1}$, Brenen W. Papenberg ${ }^{1}$, Jonathan E. Castaño ${ }^{2}$ and Scott A. Weed ${ }^{1}$

${ }^{1}$ Program in Cancer Cell Biology, Department of Biochemistry, West Virginia University, Morgantown, WV, ${ }^{2}$ Department of Otolaryngology, Head and Neck Surgery, West

Virginia University, Morgantown, WV 


\section{Abstract}

Although overall cancer incidence rates are declining across the United States, the incidence of head and neck squamous cell carcinoma (HNSCC) continues to increase within the Appalachian region. West Virginia is the only state entirely within Appalachia, with the population having increased HNSCC incidence and mortality. This underscores the need for improved understanding of Appalachian HNSCC in WV and the rest of the region in order to ultimately devise improved patient treatment. To better understand the underlying factors leading to these disproportionate outcomes, our group has established an Appalachian-specific HNSCC patient tissue cohort from surgically-resected tumors. This cohort represents all HNSCC stages, lesion types and morphologies, as well as cases that contain human papillomavirus (HPV) and/or from patients with histories of tobacco and alcohol use. Moreover, we have generated several patient derived xenografts (PDXs) from these tissues, allowing further cellular, biochemical and preclinical therapeutic evaluation. Utilization of PDX tumors from this cohort will allow examination of critical steps in the development and potential treatment of invasive, metastatic, and recurrent Appalachian-associated disease. Matched patient and PDX sample availability enables personalized medicine and co-clinical trials aimed at reversing this Appalachian cancer health disparity and ultimately improving regional HNSCC patient care. 


\section{Introduction}

Tumors of the epithelium lining the upper aerodigestive tract, termed head and neck squamous cell carcinoma (HNSCC), can form in the lips, oral cavity, nasopharynx, hypopharynx, and larynx (1-3). Anatomical location subdivides these tumors into oral cavity and pharynx or laryngeal disease, which clinically display differences in progression, response to therapy, and patient survival (1-3). Clinically HNSCC is subdivided based on human papilloma virus (HPV) infection (1-3). HPV associated HNSCC results a significantly less aggressive disease with much improved prognosis, leading the American Joint Committee on Cancer (AJCC) to down-stage all HPV-positive tumors effective January 1st, 2017 (4). Clinical trials have shown that less aggressive therapy can still garner efficacious patient outcomes in HPV-positive HNSCC (5). In contrast, prolonged tobacco and alcohol exposure is associated with HPV-negative HNSCC $(1,2)$. With diminishing tobacco consumption in the United States the number of HPV-negative HNSCC cases have decreased over time (6). Unfortunately, increasing HPV-positive incidence within this same time period has resulted in no significant change in the total number HNSCC cases during the past decade (6). Recent projections predict HPV-related HNSCC will become the predominant form of this cancer as early as 2020 (6).

Present reports indicate that HPV-positive HNSCC accounts for approximately $20 \%$ of all HNSCC incidence, but is significantly enriched in oropharyngeal tumors $(60-80 \%$ of cases) $(6-8)$. HPVpositive HNSCC is due in large part to infection of the high-risk HPV subtypes 16 and 18 (7-9). HPV is a circular double stranded DNA virus encoding 7 "early" stage proteins (E1-7) and 2 "late" stage proteins (L1 and L2) $(8,9)$. The critical early proteins E6 and E7 primarily target tumor suppressor genes p53 and $\mathrm{Rb}$ in host cells, promoting transformation of the infected epithelium and predisposing cells to subsequent genomic damage $(8,9)$. Surprisingly, HPV-positive tumors harbor similar chromosomal mutational burdens but unique mutational signatures when compared with HPV-negative tumors (10-12). Other HPV encoded genes bias these tumors 
towards a poorly differentiated and highly metastatic phenotype (13). Conversely, HPV-negative HNSCC tumors are typically well- to moderately-differentiated and undergo collective invasion causing vast loco-regional devastation resulting in decreased overall patient survival (13).

Loco-regional disease remains the focus HNSCC progression, yet metastasis to distant organs such as the lungs, bone, brain, and liver do occur $(14,15)$. More commonly, tumors spread along tissue-specific drainage pathways into the lymphatic system or follow neural pathways from the oral cavity (14-17). Both perineural invasion (PNI) and lymph node involvement result in decreased patient survival, emphasizing the necessity to control local disease prior to distal dissemination (14-17).

Utilizing 2D culture or subcutaneous and orthotopic xenograft models to model human cancer and develop pharmaceuticals has largely been ineffective, as few preclinical trial drugs duplicate their success when evaluated in the clinic (18-21). Traditional cell culture models select for highly proliferative, pro-survival subclonal cell populations presenting far greater homogeneity than found in patient tumors (21). Established cell line-based xenograft models often lack physiological tumor microenvironment interactions, displaying limited resemblance to the human disease (22). These limitations necessitate the use of more sophisticated tumor models to enhance the translatability of preclinical findings in informing patient treatment.

Genetically engineered mouse models (GEMMs) can model tumorigenesis and progression as well as serve to evaluate tumor-stroma interactions and immune surveillance of the tumor, enabling broader biological insights (23-25). These models allow researchers to address tissuespecific and temporal changes regulating key steps in tumor onset and progression that cannot be recapitulated in cell line-based xenografts $(23,24)$. GEMM studies can be used to validate novel oncogenes, evaluate new drugs and/or treatment combinations, and assess drugresistance and disease recurrence mechanisms that closely mimic the human disease $(23,25-$ 28). There are some practical and biological limitations to GEMMs in that they can be time 
consuming to generate, often display decreased tumor heterogeneity due to reliance on specific oncogenic drives rather than chromosomal instability and de novo DNA damage, and rarely capture the magnitude of alterations seen heavily pretreated patients $(27,29-31)$. While GEMMs represent marked improvement towards mimicking human disease, certain genetic abnormalities found in human patients cannot be recreated in any mouse model due to differences between species $(26,31)$. This is particularly true for large chromosomal amplification events common in human patients which have no genetic equivalent in rodents.

Patient-derived xenograft (PDX) models maintain greater tumor heterogeneity and preserve genetic landscapes found in patient tumors (30-37). Directly culturing tumors in vivo conserves several critical tumor microenvironmental facets, including reduced oxygen levels, nutrient and hormone delivery, and tumor-stromal interactions $(22,23,34)$.

PDX development begins by directly implanting patient tumor samples into immunodeficient mice, maintaining many primary patient tumor histological and genetic characteristics $(32,33,35-38)$. Patient samples are obtained as either fine needle aspirates (FNAs) or following surgical resection (34). The host mice receive either small pieces of tumor or a single-cell suspension typically mixed with pseudo-extracellular matrix (ECM) cocktail such as collagen I or Matrigel $(29,32-36,39-41)$. Tumor cells are separated into suspension through chemical digestion with ECM cleaving proteases and/or mechanical tissue mincing (29,34,40-42). These suspension techniques expose tumor cells to harsh chemical and mechanical challenges that can reduce cell viability, leading to decreased tumor engraftment and/or heterogeneity in mice (29). PDXs are inserted into heterotopic (typically subcutaneous flank or subretinal space) or orthotopic sites $(34,39,43)$. Site selection varies based on tissue sample size, orthotopic organ (both size and accessibility), and the individual PDX model purpose (tissue expansion vs. tumor phenomenon observation vs. therapeutic assessment, etc.) $(34,42)$. 
PDX engraftment success varies based on intrinsic tumor characteristics such as tumor aggressiveness, histological grade, tumor composition, sample size, resistance to anoikis and apoptosis $(29,37,38,40,43)$. Other factors that influence engraftment include time (from surgical removal from the patient to implantation in the mouse), tissue temperature, exposure to nutrients while outside of the body, and implantation location $(29,41)$.

PDX nomenclature varies but is typically denoted using either F0, G0, or P0 for initial implantation of patient tissue into the mouse $(29,34,43)$. Subsequent implantations following P0 tumor growth increase the associated passage number, indicating rounds of growth occurring within the animal model (ex. P1, P2, P3). A particular PDX sample is commonly considered established and reliable around $\mathrm{P} 3$, assuming the tumor characteristics still resemble the primary patient sample $(29,34,43,44)$. From this passage forward, PDX models are utilized in drug or tumor progression studies rather than the initial rounds of PDX establishment and tissue expansion $(29,34,43)$. Criteria for PDX tumor passaging are variable due to differing Institutional Animal Care and Use Committee (IACUC) standards and tumor monitoring techniques. Generally, once the tumor exceeds $1 \mathrm{~cm}$ in the largest dimension, the tissue is harvested, processed, then reimplanted (43). During tissue processing the tumor mass is broken down into small pieces, which can then be evaluated for tumor drift by genomic sequencing or derived into novel primary cell lines.

Several HNSCC PDX models have been developed, covering both HPV+ and HPV- subtypes. However these models represent a limited selection of HNSCC patients $(41,42,44-48)$. As seen in other tumor PDX models, engraftment rates vary based on the tissue of origin $(42,44)$. Surprisingly, while neither recurrence nor HPV status impact the success of HNSCC PDX establishment, these PDXs still recapitulate overall survival differences seen in patients $(41,42)$. The sole factor seems to influence PDX engraftment thus far is whether the patient developed metastatic disease prior to surgical resection, but this finding is inconsistent across published cohorts $(41,45-47)$. Epigenetic analysis determined that, unlike tumor cell lines, PDXs maintain 
an identical methylation signature to their primary human tumor, indicating consistent genetic regulation between the PDX and patient tumor (49). Several studies sought to identify a HNSCC cancer stem cell (CSC) subpopulation enriched for tumor initiating capacity and drug resistance $(36,40,50)$. Establishing treatment modalities to identify and target distinct subpopulations is critical to developing novel therapeutics and preventing disease progression and recurrence.

In many cases, PDX models respond to clinical therapeutics similarly to patient tumors $(37,51,52)$. Successful translation of PDX treatment to clinical efficacy enabled the National Cancer Institute $(\mathrm{NCl})$ drug screening program to replace their panel of established cell lines, known as the $\mathrm{NCl}$ 60, with a comprehensive PDX cohort (53). Recently, the Novartis Institutes for BioMedical Research unveiled a robust drug screening study utilizing its 1,075 PDX library (54). The increasing availability of large PDX cohorts should improve drug optimization and translation efforts that have largely been unsuccessful using prior methodologies. Advantageously, PDX samples can be evaluated post-treatment, identifying shifts in tumor cell protein expression that can influence secondary treatment options $(42,55)$. For instance, identifying drug resistant and susceptible subpopulations undetectable in the untreated sample provides beneficial insight towards selecting subsequent clinical interventions $(42,51,55,56)$. One study found that the drugresistant tumor subpopulation was addicted to the drug itself and regressed following treatment cessation (51). This study went on to show that periodic treatment rather than continual dosing delayed onset of drug resistance and abolished drug-reliant subpopulation development (51). Several studies have run co-clinical trials where the patient and their matched PDX are treated with identical therapeutics. Subsequent PDX sample analysis is then used to advise therapeutic selection tailored to that specific tumor (57-60). These studies utilize personalized or precision medicine, employing genomic and proteomic tumor characteristics to customize patient care rather than conventional chemotherapy, where clinicians rely upon gross tumor characteristics involving tumor size, grade, and stage to determine the optimal clinical intervention $(56,61,62)$. 
While PDXs represent advances in characterizing and preclinical testing of human tumors, there are limitations to this model. Not every tumor sample engrafts in mice. Some that do engraft can grow too slow to be practical for clinicians to adjust patient care. Additionally, mouse stromal cells replace human stromal components within the first two passages and do not always mimic behaviors found in the patient tumor microenvironment. Additionally, alterations in drug metabolism, targeting, and tumor vascularity in the mouse can weaken PDX translation to the clinical setting. More practically, PDXs come at the increased cost associated with immunodeficient mice along with a large time commitment for implanting tumors, monitoring progress, and passaging tissue. Despite these short comings, PDX preclinical and co-clinical trials enhance translational effectiveness to patient care, paving the way towards precision medicine.

West Virginia resides entirely within the Appalachian region, an area with a majority Caucasian rural population and a history of economic depression (63). While Appalachia has made remarkable strides towards attaining national averages in health care, it remains an underserved region (63). When compared to regions outside Appalachia, multiple studies indicate an increased cancer incidence and mortality within the Appalachian population $(63,64)$. West Virginia in particular shows higher total cancer incidence and a lower relative survival ratio than the national average $(64,65)$. A recent study found that while overall cancer incidence is decreasing both nationally and within Appalachia, cancers of the oral cavity/pharynx and larynx, show increasing incidence compared to the rest of the nation $(63,64)$. Specifically, there was a $20 \%$ increase in Appalachian oral cavity and pharynx cancer incidence from 2004-2011 compared to national averages $(63,64)$. This increased burden on the local and regional population necessitates a focused approach to combating this growing cancer health disparity. As a first step towards further understanding the underlying biological factors that drive Appalachian oral cavity and pharyngeal cancers, the present study details the collection and establishment of an Appalachian specific HNSCC and PDX cohort. Characterization and analysis of this cohort will begin to unravel the 
molecular and genetic mechanisms driving the disproportionate incidence in our state, enabling improved clinical treatment to reverse these trends.

\section{Materials and Methods}

\section{PDX establishment}

Patient-derived xenograft (PDX) tumors were established as described $(36,66)$. WVUSCC PDXs were derived from surgical specimens of HNSCC in compliance with the West Virginia University Institutional Review Board. PDX models were developed in compliance with the West Virginia University Institutional Animal Care and Use Committee by placing approximately $1 \mathrm{~mm}$ tumor fragments into subcutaneous pockets in the flanks of anesthetized 8-10 week-old NOD/SCID-Y (NSG) mice. Tumor fragments were overlayed with Matrigel (354234, Corning) and incisions were closed using wound clips. Mice were weighed and monitored for tumor growth on a weekly basis. PDX tumors were passaged into new NSG mice and/or used to generate cell lines once tumors reached $\sim 1 \mathrm{~cm}$ in greatest dimension.

\section{PDX-derived cell lines}

For cell line derivation, PDX tumors were minced and digested in DMEM supplemented with $20 \%$ FBS and $1 \mathrm{mg} / \mathrm{mL}$ collagenase IV (17104019, Gibco). Digested tissues were plated onto NIH 3T3 fibroblasts senesced with $4 \mu \mathrm{g} / \mathrm{mL}$ mitomycin C (BP2531, Fisher) and cultured in DMEM:F12 1:1 supplemented with $10 \%$ FBS, $400 \mathrm{ng} / \mathrm{mL}$ hydrocortisone (H0888, Sigma), $50 \mu \mathrm{g} / \mathrm{mL}$ gentamycin

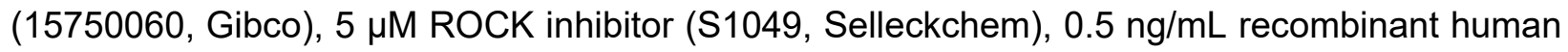
epidermal growth factor (EGF) (PHG0311, Gibco), and $10 \mathrm{ng} / \mathrm{mL}$ cholera toxin (C8062, Sigma). Prior to utilization in gelatin degradation assays, PDX derived cell lines were plated directly onto cell culture dishes for 1-2 passages to remove the fibroblast population. Gelatin degradation assays were performed in DMEM supplemented with 10\% FBS. 
Tissue sectioning, staining and microscopy

HNSCC patient and PDX tissue samples were fixed in 10\% neutral-buffered formalin and paraffin embedded (FFPE).

For patient tissue blocks, five micron sections were cut and dried onto charged glass slides at 60$65^{\circ} \mathrm{C}$ for 30 minutes. Slides were washed three times in xylene (\#8400-1, Statlabs) for two minutes each, rinsed in $100 \%, 95 \%$, and $80 \%$ ethanol (\#7100-1, Statlabs) sequentially for 20 seconds each, rinsed with distilled water twice for 10 seconds each, then incubated in hematoxylin (\#7211, Richard Allan Scientific) for 90 seconds. Slides were washed twice in distilled water for 20 seconds each, then incubated with bluing solution $(0.3 \%$ ammonium hydroxide, A669-212, Fisher Scientific) for 10 seconds and rinsed twice in distilled water for 20 seconds. Slides were washed with acid alcohol solution $(0.1 \%$ hydrochloric acid in $70 \%$ ethanol, A144-212, Fisher Scientific), twice with distilled water for 1 minute, then with $80 \%$ ethanol for 10 seconds. Slides were incubated in eosin (\#3801600, Lieca Biosystems) for 15 seconds, washed twice with 95\% ethanol, three times with $100 \%$ ethanol, then three times with xylene for 20 seconds. Slides were mounted using a Tissue-Tek SCA coverslipper (Model 4764, Sakura).

For H\&E stained PDX tumors, FFPE sections were incubated three times in microwave-heated xylene for 3 minutes each. Tumor sections were washed thrice in $100 \%$ ethanol, then once in $96 \%$ ethanol for 1 minute. Samples were washed with distilled water, incubated with hematoxylin for 30 seconds, rinsed with water for 1 minute, then with $96 \%$ ethanol. Slides were subsequently incubated with eosin for 2 minutes, washed with water for 10 seconds, then rinsed in $96 \%$ ethanol. Slides were washed with $100 \%$ ethanol, followed by three 1 minute xylene washes. Slides were dried and mounted with glass coverslips using Permount (SP15-500, Fisher). 
Histological images were acquired with an Olympus VS120 Virtual Slide microscope with a UPlanSApo 20X/0.75 objective using VS-ASW-S6 software (Olympus Corporation).

For immunofluorescent stained PDX tumors, FFPE sections were incubated three times in microwave-heated xylene for 3 minutes each. Tumor sections were washed thrice in microwaveheated $100 \%$ ethanol for 2 minutes each. Sections were then washed in $95 \%, 80 \%$, and $70 \%$ ethanol for 2 minutes each. Next sections were incubated in $11 \mathrm{mM}$ citrate buffer $\mathrm{pH} 6.0$ with $0.05 \%$ Tween 20 at $98^{\circ} \mathrm{C}$ for 20 minutes then cooled to room temperature. Slides were incubated in $1 \mathrm{X}$ PBS with $0.1 \%$ Triton $\mathrm{X}-100$ for 20 minutes followed by incubation in $1 \mathrm{X}$ PBS with $0.01 \%$ Tween 20 for 5 minutes. Tissue samples were encircled using a hydrophobic PAP pen. Antibodies used were p16 (ab2301980, 1:250, Abcam). Primary antibodies were visualized using Alexa Fluor 647 conjugated goat anti-mouse secondary antibody (A21235, 1:500, Invitrogen). F-actin was visualized with rhodamine-conjugated phalloidin (R415, 1:1000, Invitrogen). Slides were mounted using ProLong Gold antifade with DAPI (P36935, Invitrogen). Fluorescent images were acquired using a Zeiss Axiovert 200M microscope equipped with an AxioCamMR CCD camera using a Plan-Neofluar 20X/0.40 Corr objective and AxioVision software (Carl Zeiss Microscopy).

\section{Phase contrast and gelatin degradation microscopy}

Phase contrast images were acquired using a Zeiss Axiovert 200M microscope equipped with an AxioCamMR CCD camera using a Plan-Neofluar 20X/0.40 Corr objective and AxioVision software (Carl Zeiss Microscopy).

For gelatin degradation, cells were plated on Oregon Green 488-conjugated gelatin (G13186, Invitrogen) coated coverslips for $24 \mathrm{~h}$ (67). Cells were rinsed in PBS, fixed with $10 \%$ buffered formalin (SF100-4, Fisher) and labeled as described (67). Antibodies used were 4F11 (1:500). Primary antibodies were visualized using Alexa Fluor 647 conjugated goat anti-mouse secondary antibody (A21235, 1:500, Invitrogen). F-actin was visualized with rhodamine-conjugated 
phalloidin (R415, 1:1000, Invitrogen). Coverslips were mounted using ProLong Gold antifade with DAPI (P36935, Invitrogen). Gelatin degradation images were acquired with a Zeiss Axiovert 200M microscope equipped with an AxioCamMR CCD camera using a Plan-Apochromat 63X/1.4 objective and AxioVision software (Carl Zeiss Microscopy). 


\section{Results}

\section{Establishing a WVUSCC patient cohort}

In order to investigate underlying biological factors that contribute to increased HNSCC lethality in West Virginia and other Appalachia regions, a collective patient tissue repository was constructed. 63 tumor samples from different individuals encompassing the majority of HNSCC subsites were accrued from WVU Department of Pathology Laboratory for Translational Medicine. Patient consent was provided in all cases prior to surgery by the Department of OtolaryngologyHead and Neck Surgery. The cohort displays a biased occurrence towards male patients similar to that found in HNSCC cohorts from other institutions (Table 1, $(11,44,68)$ ). The vast majority of these patients present with advanced disease (Stage IV) at initial diagnosis (Table 1, $(11,44,68)$ ). Within the oropharyngeal subtype, the majority of samples came from a primary tumor, with some recurrent and metastatic patient samples (Table 1). The laryngeal subtype presented an even distribution between primary and recurrent tumors and lacked any metastatic samples (Table 1). Well differentiated tumors show clear distinction between tumor cells and the surrounding stroma at the tumor leading edge, while poorly differentiated tumors display intermixed tumor and stromal cells (69). Tumors that either fall in between those two extremes or display both morphologies across areas along the tumor leading edge are considered moderately differentiated (69). The overall WVUSCC histology matches the rank order distribution published for similar HNSCC cohorts, with the majority of patient tumors are moderately differentiated, followed by well differentiated and poorly differentiated morphology (Table 1, (68,70)). While HPV status is an important clinical parameter for patient care, HPV status for any HNSCC tumor was not routinely tested at our institution until 2017. As a result, the majority of tumors in our cohort do not have HPV status determined either by p16 immunostaining or PCR (Table 1). Importantly, over $70 \%$ of all cohort patients use tobacco in some form, with $11 \%$ having smoked with concurrent smokeless tobacco use (Table 1). Alcohol consumption is present in over $50 \%$ of cases, 
correlating with high occurrence in other studies $(11,70)$. Due to the self-reporting nature of these data, it is possible that figures for tobacco and alcohol underrepresent the number of individuals with such exposure. Overall our WVUSCC cohort contains similar representation of clinical, biological and social factors that are common to other HNSCC population groups from a highsmoking demographic.

While HNSCC represents an assortment tumors arising from various tissues, not all tissues give rise to tumors that behave uniformly in the clinic (3). Differences in outcomes and response to therapy have already been taken into account for oral cavity and pharynx vs. laryngeal disease as well as the enrichment for HPV positivity among tumors of the oropharynx $(1-3,6-8)$. To establish clarity and allow for future assessment of unique features that may arise from other tissue sites, we designated our PDX samples based on tissue of origin as defined in Cumming's Otolaryngology, $5^{\text {th }}$ edition (Table $2,(3)$ ). HNSCC is made up of five major anatomic sites: 1 ) oral cavity (OC), 2) oropharynx (OX), 3) hypopharynx (HX), 4) larynx (LX), 5) nasal cavity (NC); each of which can be further subdivided into specific tissue locations. The oral cavity comprises the lip (LP), buccal mucosa (BM), alveolar ridge (AR), floor of mouth (FM), oral tongue (OT), retromolar trigone (RT), hard palate (HP), and soft palate (SP). The oropharynx contains the base of tongue $(\mathrm{BT})$, vallecula $(\mathrm{VL})$, posterior pharyngeal wall (PPW), tonsil (TS), and lateral pharyngeal wall (LPW). The hypopharynx contains the pyriform sinus (PS) and post cricoid area (PC) (3). The larynx contains the supraglottis (SG), arytenoid (AT), epiglottis (EG), vocal cords (VC), and subglottis/infraglottis (IG). The nasal cavity contains the ethmoid sinus (ET), maxillary sinus (MS), frontal sinus (FS) and sphenoid sinus (SS). The nasopharynx (NX) contains only one subsite. Additionally, we designated associated lymph nodes as LN. Care was taken to attempt to discern the tissue of origin for each tissue collected in order to accurately account for any trends discovered from analysis of these samples. This nomenclature allows rapid determination of the specific HNSCC subsite for each patient tumor and associated PDX. 


\section{PDX Development from WVUSCC patient samples}

Detailed analysis of the genomic and proteomic landscape contributing to aggressive HNSCC in the WVUSCC cohort requires a robust tissue resource. As clinical presentation and prioritization towards pathological diagnosis limit tissue availability for further detailed analysis, tissue expansion becomes a critical step in generating sufficient tumor material for subsequent study. Sample collection begins with surgical resection where a tumor mass is excised from the patient (Fig. 1A-D). These samples undergo histological staining and pathological confirmation, with many tumors consisting of moderately differentiated cancer associated with keratin pearl formation (Fig. 1E). Keratin pearls (large pink-stained oval areas lacking cells within the tumor) are a hallmark of classical HNSCC, exhibiting the epithelial nature common to the originating tissue (Fig. 1E). Following resection, a small portion of the patient tumor is implanted into an anesthetized NSG mouse flank (Fig. 2A). Mice are monitored biweekly until tumors reach $\sim 1 \mathrm{~cm}$ in greatest diameter, at which point the mouse is sacrificed and tumor tissue is collected (Fig. 3AC). Despite the heterotopic location, established PDX tumors maintain the histological characteristics of the patient tumor from which they are derived (Fig. 4A). Clearly, the PDX tumor (Fig. 4A, left) resembles the patient tumor (Fig. 1E, left), displaying a more well differentiated morphology against the mouse stroma and continuing to present keratin pearls. Similarly, the oropharyngeal PDX tumor maintains its well differentiated status (Fig. 4A, right). While histological resemblance suggests similarities between the patient and PDX tumors, biochemical characterization is required to understand oncogenic, recurrent, and metastatic drivers within each tumor. Thus verifying expression of pathologically identified characteristics such as p16 expression is crucial to the reliability of this PDX cohort. Immunofluorescent staining of a previously pathologically identified HPV positive tumor shows sustained p16 expression in the PDX tumor, specifically staining tumor cells within the tumor mass (Fig. 4B). Together these initial indicators suggest that the PDX cohort strongly represents the WVUSCC patient cohort from 
which these samples have been established. Further genomic and proteomic examination are necessary to validate these PDX samples and will allow for insight into the causative factors driving this disease.

In addition to tumor characterization, preclinical modeling is frequently used to establish novel therapeutic approaches and paradigms aimed at improving patient outcome. To expand cell and tumor-based model availability for Appalachian HNSCC patients, PDX derived cell lines have been generated from six PDX tumors. Ongoing work will increase the number and variety of PDX derived cell lines from new tumors, enabling a more comprehensive investigation of the disease spectrum observed in the WVUSCC cohort.

For primary cell line generation, tumors excised from mice were dissociated and plated on senesced NIH 3T3 fibroblasts to generate novel cell lines. Example cell lines are shown in Fig. 5A. The WVUSCC-AR3 line, derived from a recurrent stage IV alveolar ridge tumor displays tight colony morphology in 2D co-culture typical of epithelial HNSCC cell lines (Fig. 5A left, (71)). The WVUSCC-BT4 line, derived from a recurrent stage II base of tongue tumor displays very tight colony morphology (Fig. 5A right, (71)).

As a way to measure retention of tumor behavior in the PDX cell lines, select lines were evaluated for invadopodia activity. The WVUSCC-AR3 cell line exhibits large numbers of active invadopodia (colocalization of actin and cortactin puncta at black dots within the gelatin) degrading the surrounding pseudo-ECM (Fig. 5B, top). Capturing this invasive phenomenon indicates retention of the aggressive nature of advanced stage HNSCC. The WVUSCC-BT4 cell line displays fewer invadopodia and less degradation over the same time course (Fig. 5B, bottom), suggesting lower aggressiveness as seen in p16-positive, HPV-harboring tumors. 


\section{Discussion}

The health disparity in West Virginia and other Appalachian regions for various cancers including HNSCC necessitates further investigation into the molecular and genetic drivers in this disease (65). Characterizing these patient tumors will help identify potential unique factors within Appalachia contributing to the increased incidence and mortality in this region, potentially identifying novel targets and increased information to better inform rational clinical intervention. Once the genomic and proteomic landscape for Appalachian HNSCC is known, appropriate combination therapies or novel drug development can be employed. In order to perform these detailed analysis, we generated the first Appalachian HNSCC live tissue cohort to capture the spectrum of the disease as it presents in the clinic. From there, tumor and cell-based models allow for preclinical experimentation and validation of novel approaches, tailoring patient care to the individual. This study demonstrates the creation of this cohort through the WVUCl, including a matched HNSCC PDX cohort for downstream applications. The generation of this critical resource will allow better insight into this health disparity with the end goal of resolving the Appalachian HNSCC cancer disparity through more suitable therapeutic interventions.

While the diversity within the WVUSCC cohort captures a broad spectrum within the disease, it is not representative of the total patient population treated at the WVUCl, nor does it completely extend to the local or regional population (Table 1, (65)). Patient accrual and tissue sample collection was limited to patients undergoing surgical resection whose tumors were sufficiently large enough to provide diagnostic and research samples. Even if surgical patients had adequate tumors, some patients refused consent or underwent lengthy resections which precluded tissue collection. Despite these limitations, the WVUSCC cohort captures a myriad of tumor stages, types and sublocations. There is also preferential selection for cofactors associated with poor patient outcome including advanced stage, recurrence, and metastatic disease (Table 1). Additional value of this specific HNSCC cohort lies in the unique ability to allow direct molecular, 
biological and pharmacological interrogation of tumors from patients that represent an escalating health issue among the patient population seen at the WVUCI. In addition, this cohort enables comparison between HNSCC sub-locations (oropharynx vs. larynx), stage, and differentiation status. It also encompasses the currently acknowledged extrinsic disease contributions from HPV, tobacco, and alcohol. Combining these factors allows for identification of biomarkers for oncogenesis and aggressive disease. Once these factors have been identified, preclinical experimentation aimed at combatting this disease can begin.

The successful translation of many co-clinical and preclinical drug studies carried out utilizing PDX models lends this cohort the potential for changing disease outcomes $(41,42,46-49)$. This study demonstrates PDX model reliability in maintaining hallmarks identified in patient tumors. We have shown that our PDX tumors continue to display histological characteristics (Fig. 1B vs. E) and genetic abnormalities (Fig. 1F) found in the matched patient tumor. The PDX derived cell lines enable further characterization of tumor and stromal based contributions to disease progression and recurrence. Initial novel drug compound validation in these PDX derived cell lines and tumors can assure target specificity and efficacy prior to clinical trials. Our previous work demonstrates this utility in preclinical Silmitasertib evaluation in HNSCC, a drug which other studies have concluded was ineffective $(66,72)$. Although Silmitasertib is currently ongoing clinical trials, it has yet to be evaluated in this disease as an anti-invasive therapeutic (66). Given the current paucity of targeted therapeutic options, recognition of other clinical trials or FDA approved therapies as potentially advantageous for HNSCC patients would rapidly benefit patients. Novel drug target identification will facilitate partnership with pharmaceutical research and development in order to broaden the spectrum of available treatments specifically aimed at combatting this disease. The high translatability and decreased cost of PDX preclinical studies as compared to human clinical trials can continue to lessen the burden on drug developers while at the same time enhancing the successful implementation of promising lead compounds. The continued 
expansion of this WVUSCC cohort will allow us to make more rapid advances in HNSCC treatment designed to ablate the current rising trend in HNSCC incident and mortality seen in the Appalachian patient population. 


\section{Figure Legends}

Figure 1. Representative examples of WVUSCC patient tumor anatomy and histology

A. Gross images of a recurrent stage IV laryngeal tumor involving the supraglottis, glottis, and subglottis. Scale bar represents $2.5 \mathrm{~cm}$. B. Stage IV alveolar ridge tumor exhibiting mandibular invasion. Scale bar represents $1 \mathrm{~cm}$. C. Neck mass metastasis from a stage IV oral tongue tumor. Ruler displays $\mathrm{cm}$ distance. D. Stage IV oral tongue tumor cross section. Ruler displays $\mathrm{cm}$ distance. E. Histological architecture of HNSCC patient tumors. Hematoxylin and eosin (H \& E) stained stage IV patient tumor (left) and a stage III tumor (right), both arising from the alveolar ridge. Keratin pearls are denoted with black arrows. Scale bar represents $100 \mu \mathrm{m}$ (left) and $200 \mu \mathrm{m}$ (right).

\section{Figure 2. Representative example of WVUSCC PDX implantation}

A. Image of an anesthetized NSG mouse following surgical implantation of HNSCC patient tumor tissue. Tissue that has been implanted into a subcutaneous flank pocked of a shaved/depilated mouse was covered with Matrigel and wound closed with metal surgical wound clips.

\section{Figure 3. WVUSCC PDX tumor monitoring and tissue passaging}

A. Established (P0) flank PDX tumor protruding from the mouse flank. Calipers show largest tumor dimension $(1 \mathrm{~cm})$ prior to passaging. B. WVUSCC-AR2R PDX tumors derived from recurrent stage IV patient tumor of the alveolar ridge. Two tumors are evident; one tumor mass adherent to the skin flap on either flank. C. Gross images of multiple resected PDX tumors in sterile PBS prior to mincing for reimplantation or dissociation for cell line derivation. Ruler displays cm distance.

\section{Figure 4. Histological validation of WVUSCC PDX tumors}

A. PDX tumor tissue maintains patient tumor histological architecture. H \& E stained WVUSCCAR3 P2 (left) and WVUSCC-OX3 P0 (right) PDX tumor tissue. Keratin pearls are denoted with 
black arrows. Scale bar represents $100 \mu \mathrm{m}$ (left) and $200 \mu \mathrm{m}$ (right). B. Immunofluorescent labeling of WVUSCC-BT4 PDX tissue with an anti-p16 antibody. p16 (green) expression is maintain in the PDX tumor and exclusively stains tumor cells. Merged image shows p16 along with F-actin (red) and nuclei (blue). Scale bar represents $50 \mu \mathrm{m}$.

\section{Figure 5. WVUSCC PDX-derived cell lines and gelatin degradation}

A. Phase contrast images of PDX derived cell lines grown on senesced fibroblast "feeder" cell layers. Images of the indicated WVUSCC lines are from alveolar ridge (AR, left) and base of tongue (BT, right) tumor tissue. Tumor cells display tight colony epithelial morphology indicative of moderate-well differentiated HNSCC. Scale bar represents $50 \mu \mathrm{m}$. B. Invadopodia formation and matrix degradation activity in PDX derived cell lines. Representative epifluorescent images of PDX cell lines derived from tumor tissue shown in (A). Cells were plated on OG-488 gelatin coverslips for 24 hours and labeled with an anti-cortactin antibody and rhodamine phalloidin (Actin). Gelatin is pseudo-colored white. Scale bar represents $20 \mu \mathrm{m}$. 


\section{References}

1. Rothenberg SM, Ellisen LW. The molecular pathogenesis of head and neck squamous cell carcinoma. J Clin Invest. 2012;122:1951-7.

2. Leemans $\mathrm{CR}$, Braakhuis BJM, Brakenhoff $\mathrm{RH}$. The molecular biology of head and neck cancer. Nat Rev Cancer. 2011;11:9-22.

3. Flint PW, Haughey BH, Lund VJ, Niparko JK, Richardson MA, Robbins KT, et al. Cummings Otolaryngology - Head \&amp; Neck Surgery. 5th Editio. 2010.

4. Amin MB, Edge SB, American Joint Committee on Cancer. AJCC cancer staging manual.

5. Masterson L, Moualed D, Liu ZW, Howard JEF, Dwivedi RC, Tysome JR, et al. De-escalation treatment protocols for human papillomavirus-associated oropharyngeal squamous cell carcinoma: A systematic review and meta-analysis of current clinical trials. Eur J Cancer. 2014;50:2636-48.

6. Chaturvedi AK, Engels EA, Pfeiffer RM, Hernandez BY, Xiao W, Kim E, et al. Human papillomavirus and rising oropharyngeal cancer incidence in the United States. J Clin Oncol. 2011;29:4294-301.

7. Benson E, Li R, Eisele D, Fakhry C. The clinical impact of HPV tumor status upon head and neck squamous cell carcinomas. Oral Oncol. 2014;50:565-74.

8. Rautava J, Syrjänen S. Biology of human papillomavirus infections in head and neck carcinogenesis. Head Neck Pathol. 2012;6 Suppl 1:S3-15.

9. Rampias T, Sasaki C, Psyrri A. Molecular mechanisms of HPV induced carcinogenesis in head and neck. Oral Oncol. 2014;50:356-63.

10. Keck MK, Zuo Z, Khattri A, Stricker TP, Brown CD, Imanguli M, et al. Integrative Analysis of Head and Neck Cancer Identifies Two Biologically Distinct HPV and Three Non-HPV Subtypes. Clin Cancer Res. 2015;21:870-81.

11. Seiwert TY, Zuo Z, Keck MK, Khattri A, Pedamallu CS, Stricker T, et al. Integrative and comparative genomic analysis of HPV-positive and HPV-negative head and neck squamous cell carcimonas. Clin cancer Res. 2014;

12. Zhang Y, Koneva LA, Virani S, Arthur AE, Virani A, Hall PB, et al. Subtypes of HPV-Positive Head and Neck Cancers Are Associated with HPV Characteristics, Copy Number Alterations, PIK3CA Mutation, and Pathway Signatures. Clin Cancer Res. 2016;22:4735-45.

13. O'Rorke MA, Ellison M V, Murray LJ, Moran M, James J, Anderson LA. Human papillomavirus related head and neck cancer survival: a systematic review and meta-analysis. Oral Oncol. 2012;48:1191-201.

14. Takes RP, Rinaldo A, Silver CE, Haigentz M, Woolgar JA, Triantafyllou A, et al. Distant metastases from head and neck squamous cell carcinoma. Part I. Basic aspects. Oral Oncol. 2012;48:775-9. 
15. López F, Rodrigo JP, Silver CE, Haigentz M, Bishop JA, Strojan P, et al. Cervical lymph node metastases from remote primary tumor sites. Eisele D, editor. Head Neck. 2016;38:E2374-85.

16. Kurtz KA, Hoffman HT, Bridget Zimmerman M, Robinson RA, Carver LA. Perineural and Vascular Invasion in Oral Cavity Squamous Carcinoma Increased Incidence on Re-review of Slides and by Using Immunohistochemical Enhancement. Arch Pathol Lab Med. 2005.

17. Fagan JJ, Collins B, Barnes L, D'Amico F, Myers EN, Johnson JT. Perineural Invasion in Squamous Cell Carcinoma of the Head and Neck. Arch Otolaryngol Neck Surg. American Medical Association; 1998;124:637.

18. Barretina J, Caponigro G, Stransky N, Venkatesan K, Margolin AA, Kim S, et al. The Cancer Cell Line Encyclopedia enables predictive modelling of anticancer drug sensitivity. Nature. 2012;483:603-7.

19. DiMasi JA, Reichert JM, Feldman L, Malins A. Clinical approval success rates for investigational cancer drugs. Clin Pharmacol Ther. 2013;94:329-35.

20. Hutchinson L, Kirk R. High drug attrition rates-where are we going wrong? Nat Rev Clin Oncol. 2011;8:189-90.

21. Gillet J-P, Calcagno AM, Varma S, Marino M, Green LJ, Vora MI, et al. Redefining the relevance of established cancer cell lines to the study of mechanisms of clinical anti-cancer drug resistance. Proc Natl Acad Sci U S A. 2011;108:1870813.

22. De Wever O, Mareel M. Role of tissue stroma in cancer cell invasion. J Pathol. 2003;200:429-47.

23. Gengenbacher N, Singhal M, Augustin HG. Preclinical mouse solid tumour models: status quo, challenges and perspectives. Nat Rev Cancer. Nature Publishing Group; 2017;17:751-65.

24. Day C-P, Merlino G, Van Dyke T. Preclinical mouse cancer models: a maze of opportunities and challenges. Cell. NIH Public Access; 2015;163:39-53.

25. Clohessy JG, Pandolfi PP. Mouse hospital and co-clinical trial project-from bench to bedside. Nat Rev Clin Oncol. Nature Publishing Group; 2015;12:491-8.

26. Singh M, Murriel CL, Johnson L. Genetically engineered mouse models: closing the gap between preclinical data and trial outcomes. Cancer Res. American Association for Cancer Research; 2012;72:2695-700.

27. Kersten K, de Visser KE, van Miltenburg MH, Jonkers J. Genetically engineered mouse models in oncology research and cancer medicine. EMBO Mol Med. Wiley-Blackwell; 2017;9:137-53.

28. Singh M, Lima A, Molina R, Hamilton P, Clermont AC, Devasthali V, et al. Assessing therapeutic responses in Kras mutant cancers using genetically engineered mouse models. Nat Biotechnol. Nature Publishing Group; 
2010;28:585-93.

29. Jung J, Seol HS, Chang S. The Generation and Application of Patient-Derived Xenograft Model for Cancer Research. Cancer Res Treat. 2018;50:1-10.

30. Alizadeh AA, Aranda V, Bardelli A, Blanpain C, Bock C, Borowski C, et al. Toward understanding and exploiting tumor heterogeneity. Nat Med. 2015;21:846-53.

31. Tabassum DP, Polyak K. Tumorigenesis: It takes a village. Nat Rev Cancer. Nature Publishing Group; 2015;15:473-83.

32. van Weerden WM, de Ridder CM, Verdaasdonk CL, Romijn JC, van der Kwast $\mathrm{TH}$, Schröder FH, et al. Development of seven new human prostate tumor xenograft models and their histopathological characterization. Am J Pathol. 1996;149:1055-62.

33. Reyal F, Guyader C, Decraene C, Lucchesi C, Auger N, Assayag F, et al. Molecular profiling of patient-derived breast cancer xenografts. Breast Cancer Res. 2012;14:R11.

34. Tentler JJ, Tan AC, Weekes CD, Jimeno A, Leong S, Pitts TM, et al. Patientderived tumour xenografts as models for oncology drug development. Nat Rev Clin Oncol. 2012;9:338-50.

35. Jung J, Lee CH, Seol HS, Choi YS, Kim E, Lee EJ, et al. Generation and molecular characterization of pancreatic cancer patient-derived xenografts reveals their heterologous nature. Oncotarget. 2016;7:62533-46.

36. Basu D, Nguyen T-TK, Montone KT, Zhang G, Wang L-P, Diehl JA, et al. Evidence for mesenchymal-like sub-populations within squamous cell carcinomas possessing chemoresistance and phenotypic plasticity. Oncogene. 2010;29:4170-82.

37. Sivanand S, Peña-Llopis S, Zhao H, Kucejova B, Spence P, Pavia-Jimenez A, et al. A validated tumorgraft model reveals activity of dovitinib against renal cell carcinoma. Sci Transl Med. 2012;4:137ra75.

38. John T, Kohler D, Pintilie M, Yanagawa N, Pham N-A, Li M, et al. The ability to form primary tumor xenografts is predictive of increased risk of disease recurrence in early-stage non-small cell lung cancer. Clin Cancer Res. 2011;17:134-41.

39. Morton CL, Houghton PJ. Establishment of human tumor xenografts in immunodeficient mice. Nat Protoc. 2007;2:247-50.

40. Prince ME, Sivanandan R, Kaczorowski A, Wolf GT, Kaplan MJ, Dalerba P, et al. Identification of a subpopulation of cells with cancer stem cell properties in head and neck squamous cell carcinoma. Proc Natl Acad Sci. 2007;104:973-8.

41. Kimple RJ, Harari PM, Torres AD, Yang RZ, Soriano BJ, Yu M, et al. Development and characterization of HPV-positive and HPV-negative head and neck squamous cell carcinoma tumorgrafts. Clin Cancer Res. 2013;19:855-64. 
42. Keysar SB, Astling DP, Anderson RT, Vogler BW, Bowles DW, Morton JJ, et al. A patient tumor transplant model of squamous cell cancer identifies PI3K inhibitors as candidate therapeutics in defined molecular bins. Mol Oncol. 2013;7:776-90.

43. Jin K, Teng L, Shen Y, He K, Xu Z, Li G. Patient-derived human tumour tissue xenografts in immunodeficient mice: a systematic review. Clin Transl Oncol. 2010;12:473-80.

44. Li H, Wheeler S, Park YS, Ju Z, Thomas SM, Fichera M, et al. Proteomic Characterization of Head and Neck Cancer Patient-Derived Xenografts. Mol Cancer Res. 2015;

45. Chen J, Milo GE, Shuler CF, Schuller DE. Xenograft growth and histodifferentiation of squamous cell carcinomas of the pharynx and larynx. Oral Surg Oral Med Oral Pathol Oral Radiol Endod. 1996;81:197-202.

46. Zätterström UK, Braakhuis BJ, Wennerberg J, van Dongen GA, Attewell R, Nauta $\mathrm{JJ}$, et al. Growth of xenografted squamous cell carcinoma of the head and neck-possible correlation with patient survival. APMIS. 1992;100:976-80.

47. Wennerberg J, Tropé C, Biörklund A. Heterotransplantation of human head and neck tumours into nude mice. Acta Otolaryngol. 1983;95:183-90.

48. Langdon SP, Hendriks HR, Braakhuis BJ, Pratesi G, Berger DP, Fodstad O, et al. Preclinical phase II studies in human tumor xenografts: a European multicenter follow-up study. Ann Oncol Off J Eur Soc Med Oncol. 1994;5:415-22.

49. Hennessey PT, Ochs MF, Mydlarz WW, Hsueh W, Cope L, Yu W, et al. Promoter methylation in head and neck squamous cell carcinoma cell lines is significantly different than methylation in primary tumors and xenografts. Wang XW, editor. PLoS One. 2011;6:e20584.

50. Basu D, Montone KT, Wang L-P, Gimotty PA, Hammond R, Diehl JA, et al. Detecting and targeting mesenchymal-like subpopulations within squamous cell carcinomas. Cell Cycle. 2011;10:2008-16.

51. Das Thakur M, Salangsang F, Landman AS, Sellers WR, Pryer NK, Levesque $\mathrm{MP}$, et al. Modelling vemurafenib resistance in melanoma reveals a strategy to forestall drug resistance. Nature. 2013;494:251-5.

52. Merk J, Rolff J, Becker M, Leschber G, Fichtner I. Patient-derived xenografts of non-small-cell lung cancer: a pre-clinical model to evaluate adjuvant chemotherapy? Eur J Cardiothorac Surg. 2009;36:454-9.

53. Ledford $\mathrm{H}$. US cancer institute to overhaul tumour cell lines. Nature. 2016;530:391-391.

54. Gao H, Korn JM, Ferretti S, Monahan JE, Wang Y, Singh M, et al. Highthroughput screening using patient-derived tumor xenografts to predict clinical trial drug response. Nat Med. 2015;21:1318-25.

55. Migliardi G, Sassi F, Torti D, Galimi F, Zanella ER, Buscarino M, et al. Inhibition of 
MEK and PI3K/mTOR suppresses tumor growth but does not cause tumor regression in patient-derived xenografts of RAS-mutant colorectal carcinomas. Clin Cancer Res. 2012;18:2515-25.

56. Erriquez J, Olivero M, Mittica G, Scalzo MS, Vaira M, De Simone M, et al. Xenopatients show the need for precision medicine approach to chemotherapy in ovarian cancer. Oncotarget. 2016;7:26181-91.

57. Garrido-Laguna I, Tan AC, Uson M, Angenendt M, Ma WW, Villaroel MC, et al. Integrated preclinical and clinical development of mTOR inhibitors in pancreatic cancer. Br J Cancer. 2010;103:649-55.

58. Jimeno A, Amador ML, Kulesza $P$, Wang X, Rubio-Viqueira $B$, Zhang $X$, et al. Assessment of celecoxib pharmacodynamics in pancreatic cancer. Mol Cancer Ther. 2006;5:3240-7.

59. Lunardi A, Ala U, Epping MT, Salmena L, Clohessy JG, Webster KA, et al. A coclinical approach identifies mechanisms and potential therapies for androgen deprivation resistance in prostate cancer. Nat Genet. 2013;45:747-55.

60. Owonikoko TK, Zhang G, Kim HS, Stinson RM, Bechara R, Zhang C, et al. Patient-derived xenografts faithfully replicated clinical outcome in a phase II coclinical trial of arsenic trioxide in relapsed small cell lung cancer. J Transl Med. 2016;14:111.

61. Gonçalves A, Bertucci F, Guille A, Garnier S, Adelaide J, Carbuccia N, et al. Targeted NGS, array-CGH, and patient-derived tumor xenografts for precision medicine in advanced breast cancer: a single-center prospective study. Oncotarget. 2016;7:79428-41.

62. Witkiewicz AK, Balaji U, Eslinger C, McMillan E, Conway W, Posner B, et al. Integrated Patient-Derived Models Delineate Individualized Therapeutic Vulnerabilities of Pancreatic Cancer. Cell Rep. 2016;16:2017-31.

63. Wilson RJ, Ryerson AB, Singh SD, King JB. Cancer Incidence in Appalachia, 2004-2011. Cancer Epidemiol Biomarkers Prev. 2016;25:250-8.

64. Cancer in North America: 2011-2015. 2018.

65. 2017 West Virginia Cancer Burden Report. Morgantown; 2017.

66. Markwell SM, Ammer AG, Interval ET, Allen JL, Papenberg RA, Castano JE, et al. Cortactin Phosphorylation by Casein Kinase 2 Regulates Actin-Related Protein 2/3 Complex Activity, Invadopodia Function and Tumor Cell Invasion. Mol cancer Res. 2019;in press.

67. Martin KH, Hayes KE, Walk EL, Ammer AG, Markwell SM, Weed SA. Quantitative Measurement of Invadopodia-mediated Extracellular Matrix Proteolysis in Single and Multicellular Contexts. J Vis Exp. 2012;e4119.

68. Weller P, Bankfalvi A, Gu X, Dominas N, Lehnerdt GF, Zeidler R, et al. The role of tumour FoxP3 as prognostic marker in different subtypes of head and neck 
cancer. Eur J Cancer. 2014;50:1291-300.

69. Rivera C, Venegas B. Histological and molecular aspects of oral squamous cell carcinoma. Oncol Lett. 2014;8:7-11.

70. Chang K-P, Kao H-K, Yen T-C, Chang Y-L, Liang Y, Liu S-C, et al. Overexpression of macrophage inflammatory protein-3alpha in oral cavity squamous cell carcinoma is associated with nodal metastasis. Oral Oncol. 2011;47:108--113.

71. Zhao M, Sano D, Pickering CR, Jasser SA, Henderson YC, Clayman GL, et al. Assembly and initial characterization of a panel of 85 genomically validated cell lines from diverse head and neck tumor sites. Clin Cancer Res. 2011;17:7248-64.

72. Bian Y, Han J, Kannabiran V, Mohan S, Cheng H, Friedman J, et al. MEK inhibitor PD-0325901 overcomes resistance to CK2 inhibitor CX-4945 and exhibits antitumor activity in head and neck cancer. Int J Biol Sci. Ivyspring International Publisher; 2015;11:411-22. 
Figures and Tables
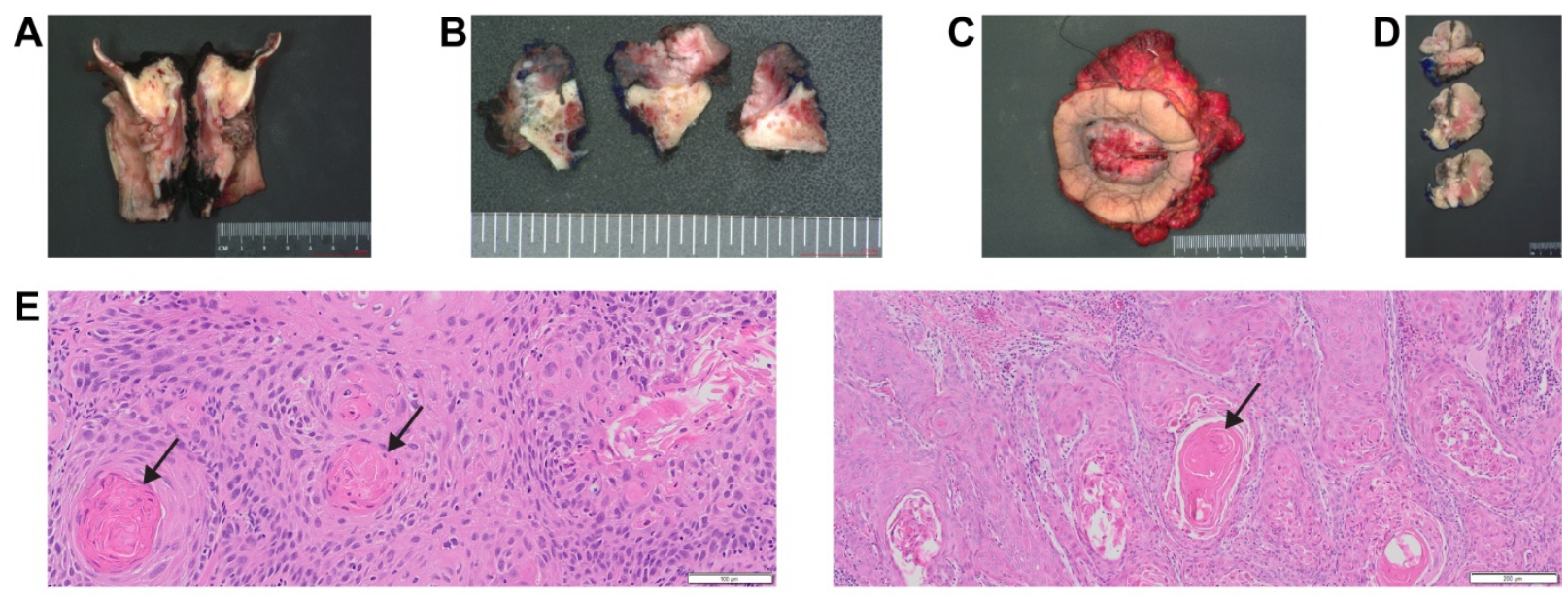

Figure 1 Markwell et al. 


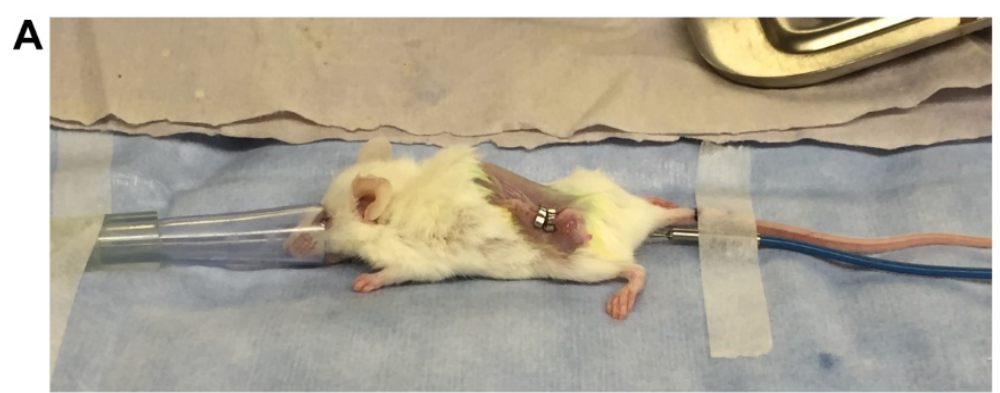

Figure 2 Markwell et al. 


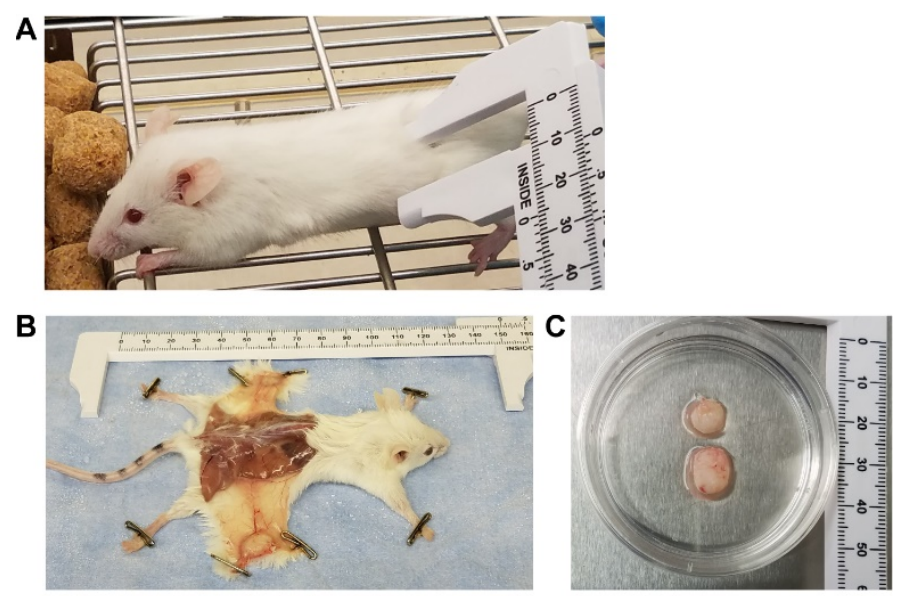

Figure 3 Markwell et al. 

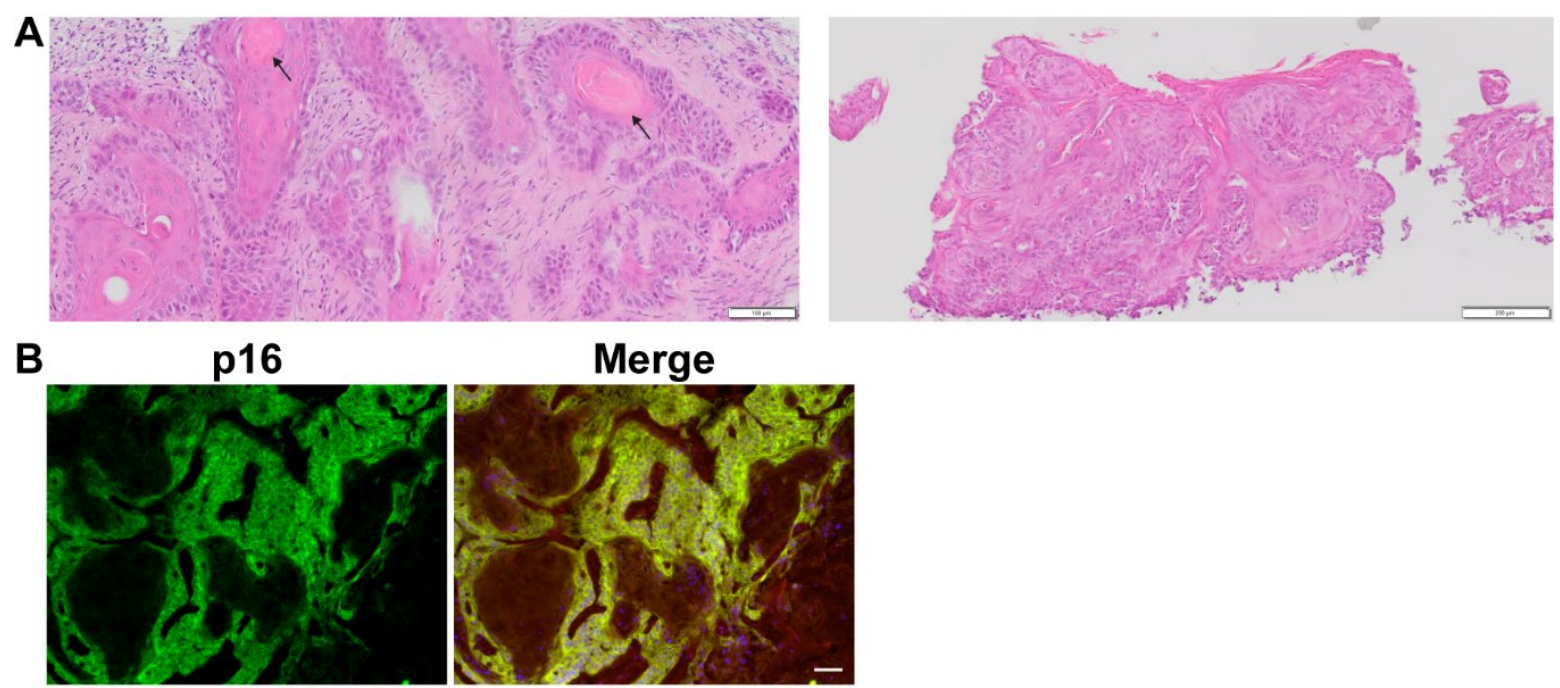

Figure 4 Markwell et al. 

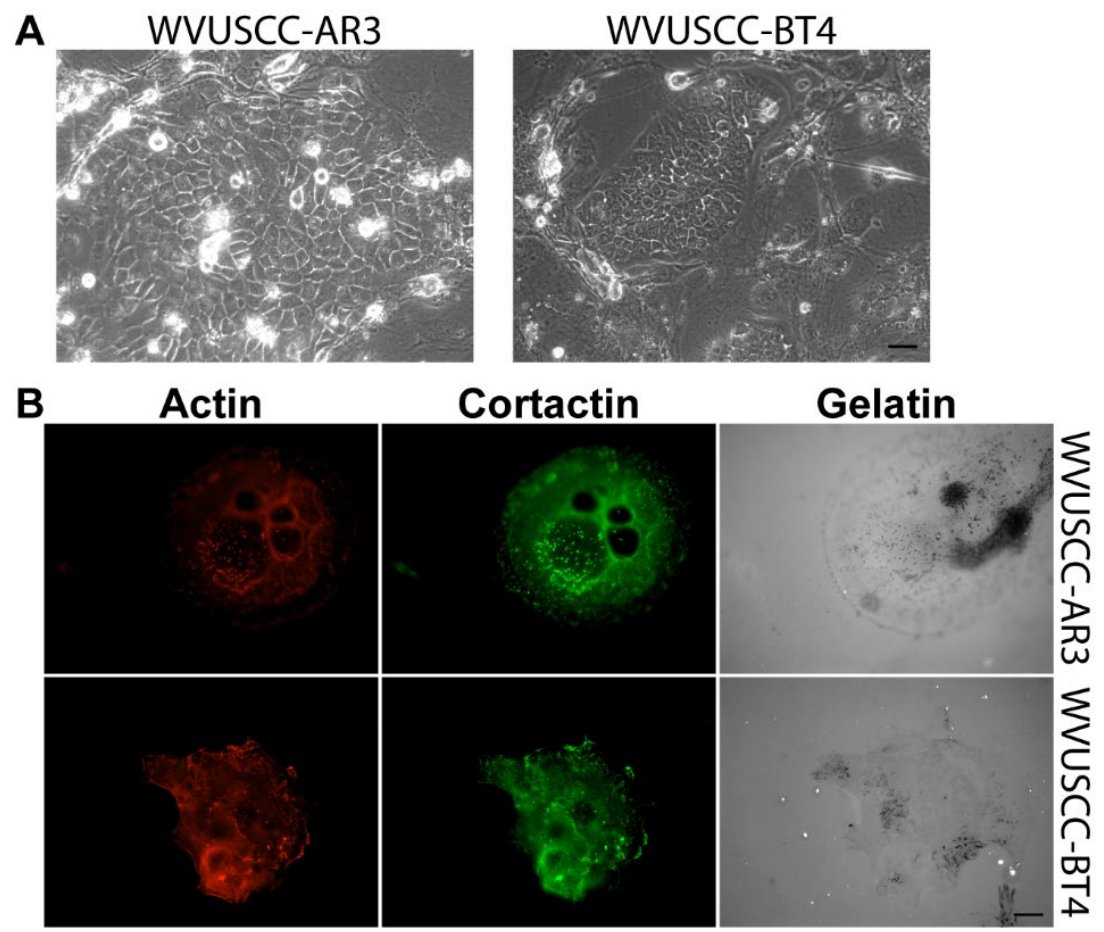

Figure 5 Markwell et al. 
Table 1. WVUSCC patient cohort

\begin{tabular}{|c|c|c|c|}
\hline Characteristic & $\begin{array}{l}\text { All } \\
n=63 \\
(100 \%)\end{array}$ & $\begin{array}{l}\text { Oropharynx } \\
n=53 \\
(100 \%)\end{array}$ & $\begin{array}{c}\text { Larynx } \\
n=10 \\
(100 \%)\end{array}$ \\
\hline & $\mathrm{n}(\%)$ & $\mathrm{n}(\%)$ & $\mathrm{n}(\%)$ \\
\hline \multicolumn{4}{|l|}{ Sex } \\
\hline Male & $43(68.25)$ & $36(67.92)$ & $7(70)$ \\
\hline Female & $20(31.75)$ & $17(32.08)$ & $3(30)$ \\
\hline \multicolumn{4}{|l|}{ AJCC Stage } \\
\hline I / II & $13(20.63)$ & $12(22.64)$ & $1(10)$ \\
\hline III & $8(12.7)$ & $5(9.43)$ & $3(30)$ \\
\hline IV & $42(66.67)$ & $36(67.92)$ & $6(60)$ \\
\hline \multicolumn{4}{|l|}{ Type of Lesion } \\
\hline Primary & $40(63.49)$ & $35(66.04)$ & $5(50)$ \\
\hline Recurrence & $21(33.33)$ & $16(30.19)$ & $5(50)$ \\
\hline Metastasis & $2(3.17)$ & $2(3.77)$ & - \\
\hline \multicolumn{4}{|c|}{ Differentiation Status } \\
\hline Well & $15(23.81)$ & $12(22.64)$ & $3(30)$ \\
\hline Moderate & $38(60.32)$ & $32(60.38)$ & $6(60)$ \\
\hline Poor & $7(11.11)$ & $6(11.32)$ & $1(10)$ \\
\hline Unknown & $2(3.17)$ & $2(3.77)$ & - \\
\hline \multicolumn{4}{|l|}{ HPV Status } \\
\hline Positive & $9(14.29)$ & $9(16.98)$ & - \\
\hline Negative & $16(25.4)$ & $15(28.3)$ & $1(10)$ \\
\hline Missing & $38(60.32)$ & $29(54.72)$ & $9(90)$ \\
\hline \multicolumn{4}{|l|}{ Tobacco Usage } \\
\hline Smoking & $45(71.43)$ & $35(66.04)$ & $10(100)$ \\
\hline Smokeless & $14(22.22)$ & $14(26.42)$ & - \\
\hline Both & $7(11.11)$ & $7(13.21)$ & - \\
\hline Neither & $6(9.52)$ & $6(11.32)$ & - \\
\hline \multicolumn{4}{|l|}{ Alcohol Usage } \\
\hline Yes & $36(57.14)$ & $31(58.49)$ & $5(50)$ \\
\hline Never & $25(39.68)$ & $20(37.74)$ & $5(50)$ \\
\hline Unknown & $2(3.17)$ & $2(3.77)$ & - \\
\hline
\end{tabular}

Markwell et al. Table 1 
Table 2. WVUSCC tissue naming convention

\begin{tabular}{|lcc|}
\hline Subsite & Abbreviation & Anatomic Site \\
\hline Lip & LP & Oral Cavity \\
Buccal mucosa & BM & \\
Alveolar ridge & AR & \\
Floor of mouth & FM & \\
Oral tongue & OT & \\
Retromolar trigone & RT & \\
Hard palate & HP & \\
Soft palate & SP & \\
\hline Lymph node & LN & \\
\hline Oropharynx & OX & Oropharynx \\
Base of tongue & BT & \\
Vallecula & VL & \\
Posterior pharyngeal wall & PPW & \\
Tonsil & TS & \\
Lateral pharyngeal wall & LPW & \\
\hline Nasopharynx & NX & \\
\hline Hypopharynx & HX & Hypopharynx \\
Pyriform sinus & PS & \\
Post cricoid area & PC & \\
\hline Larynx & LX & Larynx \\
Supraglottis & SG & \\
Arytenoid & AT & \\
Epiglottis & EG & \\
Vocal cord & VC & \\
Subglottis & IG & for Infraglottis \\
\hline Nasal cavity & NC & Nasal Cavity \\
Ethmoid sinus & ET & \\
Maxillary sinus & MS & \\
Frontal sinus & FS & \\
Sphenoid sinus & SS & \\
\hline
\end{tabular}

Markwell et al. Table 2 


\section{GENERAL DISCUSSION}

This dissertation describes studies that elucidate a novel phospho-regulatory pathway that modulates tumor cell invasiveness and the development of a unique Appalachian HNSCC patient cohort with matched PDX samples. The first study details a druggable target that regulates the initial steps of cellular invasion which controls tumor invasion in an orthotopic setting. The second study establishes a comprehensive tissue collection allowing investigation of significant steps in the development and potential treatment of invasive, metastatic, and recurrent Appalachianassociated HNSCC. Collectively, these studies expand potential treatment options for HNSCC patients and position us to uncover unique facets driving higher HNSCC incidence and poor outcome among Appalachian patients as initial steps to combat this cancer health disparity.

While HNSCC loco-regional and distal spread has long been shown to coincide with decreased patient survival, we are just beginning to understand the various modes of invasion enabling lymphatic and metastatic dissemination (1-5). Tumor cell invasion occurs across a spectrum, ranging from single-cell modalities to large multi-cellular structures $(6,7)$. These collective invasive groups often contain stromal cells alongside tumor cells, leading to greater appreciation for the contributions of the tumor microenvironment toward tumor progression $(7,8)$. Currently, there are promising new therapeutic interventions targeting tumor stromal components that target endothelial cells, CAFs, and TAMs along with various immunotherapies aimed at reinitiating immune surveillance (9-12). As future studies expand our understanding of stromal contributions to tumorigenesis, progression and recurrence, it seems likely that combination therapies will soon include components aimed at combating multiple facets of the tumor microenvironment.

At the cellular level, many studies have focused on tumor cell-based motility and invasion, much of which relies on actin cytoskeletal dynamics allowing for concerted cell migration. The interaction between Arp2/3 complex and associate NPFs, mainly N-WASp and cortactin, are critical to producing the F-actin network at the center of migratory and invasive cellular 
substructures such as lamellipodia, filapodia, podosomes and invadopodia (13-15). These structures require tightly regulated actin dynamics achieved through specific interactions among various ABPs (13-15). Cortactin plays a key role maintaining the integrity of the F-actin network through its ability to stabilize actin branch point through interactions in the NTA domain and RR (16). Much of the current literature focuses on cortactin as a central integrator for multiple upstream signaling pathways that converge on actin cytoskeletal regulation. Post-translational modifications to the cortactin C-terminal end have been established for Src, Erk1/2, PAK family kinases, PKD, and Arg/Abl family kinases (17). All of these modifications are suggested to release cortactin from an auto-inhibited state, opening the protein up to become a scaffold for other ABPs (17). Less is known regarding signaling events that modulate the ability of cortactin to interact with other binding partners. Several studies have demonstrated that removal of the DDW motif in the cortactin NTA domain dramatically reduces binding to Arp2/3 complex and subsequently blunts invadopodia formation and maturation in multiple cell lines (18-25). Data presented in Study 1 demonstrate a novel CK2-cortactin signaling mechanism which is required for efficient invadopodia formation and function leading to tumor cell invasion in 3D and in vivo. We observed that CK2-mediated cortactin NTA phosphorylation proximal to the DDW motif is sufficient to diminish binding to and/or activation of Arp2/3 complex. This is in agreement with the majority of the literature that indicates CK2 phosphorylation plays an inhibitory role with respect to other NPFs (26-29).

Despite significant reductions in HNSCC tumor cell invadopodia and invasion due to modification of cortactin T24 and CK2 inhibition, invadopodia or tumor cell invasion were not completely abolished (Study 1). The incomplete ability of cortactin removal or CK2 inhibition to block invasion has not been emphasized in other work (30-36). Unlike CK2 inhibition, which has mainly been studied with a focus on anti-proliferative effects, cortactin inhibition resulting in reduced invasiveness has been extensively documented in many tumor cell types. Results from across 
these studies have shown significant reduction in invadopodia and invasion following reduced cortactin expression, yet some cells still retain the ability to form functional invadopodia (16,3033). Evaluation of this work indicates that $\sim 20 \%$ of cells still make invadopodia and degrade matrix when cortactin is directly targeted. While it is possible that this residual activity results from incomplete cortactin reduction through limitations of RNAi, the consistent lower limit achieved among multiple laboratories (including our own) using a variety of cell lines suggests that tumor cells also possess cortactin-independent invasion mechanism(s) to ensure invadopodia are produced. Studies utilizing CRISPR-Cas9 technologies or cortactin null GEMM models can be used to verify the existence of such a mechanism. Presuming that such a mechanism does exist, there are several ways to elucidate the compensatory pathways allowing cortactin-independent invasion. Following the creation of cortactin-null cell lines, RNA sequencing and proteomic analysis may reveal compensatory upregulation of proteins able to stabilize actin networks in the absence of cortactin. We have already tested our cortactin KD cell lines for expression of the cortactin homologue HS1 and found that all HNSCC cell lines tested failed to upregulate HS1. Upregulation or a change in the phosphorylation status of other ABPs will generate a list of targets for subsequent investigation. Utilizing an RNA or CRISP-mediated reduction in those target proteins in cortactin-null cells will be helpful in further validating the comprehensive network involved in compensatory cortactin-independent invasive processes. Such targets can serve as the basis for additional anti-invasive therapeutic development in order to generate a combination therapy capable of fully inhibiting tumor invasion. It is also possible, if not likely, that there are tumor cell independent compensatory mechanisms in vivo that can facilitate tumor cell invasion in the absence of cortactin. This is supported by the fact that cortactin amplification and overexpression only occurs in a subset of tumors, indicating that tumors can progress without relying on cortactin driven enhancements $(1,30,33,37-45)$. The most likely compensation in this case would arise from recruitment of various stromal cells to the tumor border, followed by stromal-cell directed invasion. This can already be seen in certain tumors undergoing collective 
invasion mediated through the proteolytic action of CAFs on the ECM to generate permissive trails for tumor cells for migration independent of tumor cell-driven ECM proteolysis $(6,7)$. In addition to CAFs, other immune infiltrating cells such as TAMs are capable of remodeling the ECM and can similarly contribute to generating a permissive environment for cortactinindependent tumor cell invasion. Further stromal cell contributions to tumor cell invasion are detailed in Markwell and Weed 2015 which can be found under Appendix items attached to end of this dissertation (p.172-196).

CK2 has been established as a pro-oncogenic gene involved in cell survival, proliferation and resistance to apoptosis (46). In agreement with a pro-invasive role, CK2 is enriched at the tumor border and correlates with enhanced tumor cell invasion and metastatic progression in various tumor types $(47,48)$. Many signaling pathways require CK2 activity for optimal signal transduction including mitogenic signals, inflammatory cytokines, apoptosis, cell survival and angiogenesis (49-54). The central role of CK2 in multiple important oncogenic process spurred the development of many pharmacological inhibitors, though only a select few have shown promise as true clinical interventions (55). Data from Study 1 demonstrate efficacy for Silmitasertib in blunting HNSCC invasion in 3D and in vivo models. While insufficient as a single agent, CK2 inhibition may be a useful component of a multi-drug regimen for treating aggressive tumors. Several preclinical and early clinical studies have already demonstrated a synergistic role for CK2 inhibition when in combination with other standard chemotherapeutic agents, mainly through sensitizing cells to apoptosis (56-58). Targeting the CK2-cortactin signal pathway with Silmitasertib presents a new potential anti-invasion therapeutic option for HNSCC patients. Preclinical efficacy in PDX-derived cell lines further suggests the likelihood of clinical benefit resultant from Silmitasertib administration, since PDXs have been shown to exhibit similar response to patient tumors when treated with the same agents (59-61). 
While data presented in Study 1 identify CK2 $\alpha$ as the T24 phosphorylating kinase, future efforts are needed to discover the pT24 phosphatase capable of regenerating T24 cortactin that is required for optimal interaction with Arp2/3 complex. The Slingshot family phosphatases are potential candidates for this role, since they localize to actin-rich structures and are capable of dephosphorylating other ABPs including cofilin and coronin (62-64). The close proximity Slingshot phosphatases to sites of cortactin activity suggest that it may be capable of acting upon pT24 in the cortactin NTA domain. Alternatively, CK2 $\alpha$ has been shown to bind to and enhance the activity of protein phosphatase 2A (PP2A) in fibroblasts and glioma cell lines $(65,66)$. PP2A directly binds to the CK2 catalytic subunit and blocks its association with the regulatory $\beta$ subunit, preventing CK2 holoenzyme formation (66). The feasibility of this interaction at invadopodia warrants further investigation, as the CK2 $\beta$ subunit localizes the holoenzyme to the plasma membrane and may be critical for CK2 association at areas of actin network formation in invadopodia $(67,68)$. It is possible that both interactions with CK2 $\alpha$ are coordinated to enhance CK2 signal cycling. One interaction allowing for $\mathrm{CK} 2 \alpha$ substrate phosphorylation is through proper localization of the kinase domain, while a second enhances substrate dephosphorylation. These two events combine to form a CK2 phosphoregulatory cycle at the plasma membrane. Additionally, RNA sequencing or proteomic analysis of CK2 overexpressing cell lines and patient tumors could generate an unbiased approach to identifying likely CK2 phosphatase candidates. These candidate genes could then be verified through biochemical, RNAi, or phosphatase inhibitor studies in order to identify candidate phosphatases that contribute to the CK2-cortactin phosphoregulatory cycle described herein.

PDX models maintain greater tumor heterogeneity and preserve genetic landscapes found in patient tumors $(59,69-75)$. Directly culturing tumors in vivo conserves several critical tumor microenvironmental facets, including reduced oxygen levels, nutrient and hormone delivery, and tumor-stromal interactions $(71,76,77)$. Despite some failures of PDX-based treatment outcomes 
to recapitulate their results in the clinical setting, PDX models have far superior success rates when compared to cell culture and animal-based studies (78). Not only do PDX models allow for repurposing current FDA approved drugs and the generation of novel combinational therapies with those drugs, but PDX tissue analysis post treatment allows for discovery of new druggable targets for further research and development $(79,80)$. The success of a small handful of co-clinical trials has already altered the way that innovative oncologists approach patient care. The current shift toward rapid, reliable sequencing utilizing personalized or precision medicine through genomic and proteomic tumor analysis will allow customized patient care to become more frequently utilized in clinical practice in the future.

Although PDX models are becoming increasingly popular tools for studying tumor behavior, this model still does not fully recapitulate the human disease. Rapid depletion of human stromal cells with their mouse stromal counterparts that do not entirely mimic behaviors found in the patient tumor microenvironment limits the utility of PDX models. Furthermore, the immune compromised nature of these animals prevents observation of tumor-immune cell interactions. The inability to observe stromal and immune cell compensation in the tumor microenvironment is the greatest limitation to PDX-based discoveries. Additionally, differences in drug metabolism, targeting, and tumor vascularity in the mouse diminishes PDX translation to the clinical setting. Development of better immune-compromised mouse hosts such as humanized mice and more sophisticated de novo tumorigenesis models will enable researchers to recapitulate disease development, progression, response to therapy and recurrence.

The Appalachian population displays increased cancer incidence and mortality when compared to the rest of the United States $(81,82)$. West Virginia specifically exhibits higher total cancer incidence and a lower relative survival ratio than the national average $(81,83)$. Despite a decrease in overall cancer incidence at the national level and within Appalachia, cancers of the oral cavity/pharynx and larynx, continue to have higher incidence in Appalachia $(81,82)$. The 
enhanced tumor burden on the local and regional population warrants directed efforts to reverse this cancer health disparity. Our group has begun such an effort towards further understanding biological factors that drive Appalachian oral cavity and pharyngeal cancers through the collection and establishment of an Appalachian specific HNSCC and PDX cohort. We have amassed 63 tumor samples covering the majority of HNSCC subsites across all stages, histologic subtypes and known causative factors including HPV, tobacco and alcohol exposure. The established PDX tissues maintain histological and functional similarities to the patient samples from which they are derived (Study 2). Characterization and future investigation of this cohort seeks to reveal the molecular and genetic mechanisms driving the disproportionate incidence and mortality in West Virginia and neighboring Appalachian regions. Successful analysis will enable informed clinical care to combat this health disparity. Additional investigation into the genomic and proteomic topography within Appalachian HNSCC combined with current array of PDXs will allow for rapid evaluation of novel treatments directed towards improving HNSCC patient survival. With the limited targeted therapeutic agents available in HNSCC, any novel interventions will provide meaningful advances in patient care.

As noted in the discussion section of Study 1, there are several subsequent studies required to further confirm these findings. Successful pT24 antibody development would enable direct detection of CK2-mediated cortactin regulation. This would confirm that CK2 acts directly on cortactin in cell lines and tumor tissues. It would also allow analysis of cortactin amplified and overexpressing tumors to determine if pT24 levels correlate with enhanced invasiveness, metastasis, and poor outcome as predicted by this dissertation. Additional analysis directed at observing invadopodia dynamics in real time could determine whether the T24 phosphoregulatory cycle alters invadopodia initiation or maturation. This can be partially achieved through actin incorporation assays aimed at labeling actin barded ends in areas of invadopodia development. A complementary method involves live cell imaging of de novo invadopodia 
formation and maturation utilizing $2 \mathrm{D}$ or $3 \mathrm{D}$ invasion models. Combined these data would elucidate the exact step(s) of invadopodia formation and function that CK2 phosphorylation regulate.

Further validation of Silmitasertib in blunting tumor invasiveness can be achieved through additional animal modeling. PDX tumor treatment either at heterotopic or orthotopic sites would confirm its utility in treating advanced human HNSCC tumors. However, PDX models and the orthotopic tumor injection model shown in Study 1 all start treatment following significant tumor establishment. Further studies involving spontaneous tumor development combined with Silmitasertib treatment would determine the extent to which CK2 inhibition can blunt tumor progression from the point of tumor initiation. Focusing solely on CK2-dependent processes, additional studies carried on patients or animals with post-surgical tumor resection may demonstrate Silmitasertib efficacy towards delaying or even preventing disease recurrence. As has been revealed with CK2 inhibition in other tumor types, Silmitasertib would be expected to enhance HNSCC tumor sensitivity to cytotoxic chemotherapies. Thus, Silmitasertib could be a component of first-line therapeutic combinations. In the case of HNSCC, it has yet to be determined if CK2 inhibition enhances EGFR inhibition. As EGFR blockade is the only tumor targeted therapy in HNSCC that shows efficacy in the metastatic setting (84), it would be interesting to evaluate any potential synergistic overlap between these two major signaling pathways through combined Silmitasertib and Erbitux treatment in the neoadjuvant or postoperative settings. 


\section{References}

1. Martin CL, Reshmi SC, Ried T, Gottberg W, Wilson JW, Reddy JK, et al. Chromosomal imbalances in oral squamous cell carcinoma: examination of 31 cell lines and review of the literature. Oral Oncol. 2008;44:369-82.

2. Kurtz KA, Hoffman HT, Bridget Zimmerman M, Robinson RA, Carver LA. Perineural and Vascular Invasion in Oral Cavity Squamous Carcinoma Increased Incidence on Rereview of Slides and by Using Immunohistochemical Enhancement. Arch Pathol Lab Med. 2005.

3. Fagan JJ, Collins B, Barnes L, D'Amico F, Myers EN, Johnson JT. Perineural Invasion in Squamous Cell Carcinoma of the Head and Neck. Arch Otolaryngol Neck Surg. American Medical Association; 1998;124:637.

4. Takes RP, Rinaldo A, Silver CE, Haigentz M, Woolgar JA, Triantafyllou A, et al. Distant metastases from head and neck squamous cell carcinoma. Part I. Basic aspects. Oral Oncol. 2012;48:775-9.

5. López F, Rodrigo JP, Silver CE, Haigentz M, Bishop JA, Strojan P, et al. Cervical lymph node metastases from remote primary tumor sites. Eisele D, editor. Head Neck. 2016;38:E2374-85.

6. Friedl P, Locker J, Sahai E, Segall JE. Classifying collective cancer cell invasion. Nat Cell Biol. 2012;14:777-83.

7. Friedl P, Alexander S. Cancer invasion and the microenvironment: plasticity and reciprocity. Cell. Elsevier Inc.; 2011;147:992-1009.

8. Markwell S, Weed S. Tumor and Stromal-Based Contributions to Head and Neck Squamous Cell Carcinoma Invasion. Cancers (Basel). 2015;7:382-406.

9. Kosoff D, Lang JM. Development and translation of novel therapeutics targeting tumorassociated macrophages. Urol Oncol Semin Orig Investig. 2018;

10. Chen X, Song E. Turning foes to friends: targeting cancer-associated fibroblasts. Nat Rev Drug Discov. 2018;

11. Vassilakopoulou M, Psyrri A, Argiris A. Targeting angiogenesis in head and neck cancer. Oral Oncol. 2015;

12. Jie H-B, Srivastava RM, Argiris A, Bauman JE, Kane LP, Ferris RL. Increased PD-1+ and TIM-3+ TILs during cetuximab therapy inversely correlates with response in head and neck cancer patients. Cancer Immunol Res. 2017;canimm.0333.2016.

13. Rottner K, Faix J, Bogdan S, Linder S, Kerkhoff E. Actin assembly mechanisms at a glance. J Cell Sci. 2017;130:3427-35.

14. Yamaguchi $\mathrm{H}$, Condeelis $\mathrm{J}$. Regulation of the actin cytoskeleton in cancer migration and invasion. Biochem Biophys Acta. 2007;1773:642-52.

15. Siton-Mendelson O, Bernheim-Groswasser A. Functional Actin Networks under Construction: The Cooperative Action of Actin Nucleation and Elongation Factors. Trends Biochem Sci. 2017;42:414-30.

16. Markwell SM, Ammer AG, Interval ET, Allen JL, Papenberg RA, Castano JE, et al. 
Cortactin Phosphorylation by Casein Kinase 2 Regulates Actin-Related Protein 2/3 Complex Activity, Invadopodia Function and Tumor Cell Invasion. Mol cancer Res. 2019;in press.

17. Ayala I, Baldassarre M, Giacchetti G, Caldieri G, Tete S, Luini A, et al. Multiple regulatory inputs converge on cortactin to control invadopodia biogenesis and extracellular matrix degradation. J Cell Sci. 2008;121:369-78.

18. Weaver AM, Heuser JE, Karginov A V., Lee W, Parsons JT, Cooper JA. Interaction of cortactin and N-WASp with Arp2/3 complex. Curr Biol. 2002;12:1270-8.

19. Pollard TD. Regulation of Actin Filament Assembly by Arp2/3 Complex and Formins. Annu Rev Biophys Biomol Struct. Annual Reviews; 2007;36:451-77.

20. Miglarese MR, Mannion-Henderson J, Wu H, Parsons JT, Bender TP. The protein tyrosine kinase substrate cortactin is differentially expressed in murine $B$ lymphoid tumors. Oncogene. 1994;9:1989-97.

21. Du Y, Weed SA, Xiong WC, Marshall TD, Parsons JT. Identification of a novel cortactin $\mathrm{SH} 3$ domain-binding protein and its localization to growth cones of cultured neurons. Mol Cell Biol. 1998;18:5838-51.

22. Weed S a, Du Y, Parsons JT. Translocation of cortactin to the cell periphery is mediated by the small GTPase Rac1. J Cell Sci. 1998;111 ( Pt 1:2433-43.

23. Daly RJ. Cortactin signalling and dynamic actin networks. Biochem J. 2004;382:13-25.

24. Weed SA, Karginov A V., Schafer DA, Weaver AM, Kinley AW, Cooper JA, et al. Cortactin localization to sites of actin assembly in lamellipodia requires interactions with F-actin and the Arp2/3 complex. J Cell Biol. 2000;151:29-40.

25. Galletta BJ, Chuang DY, Cooper JA. Distinct roles for Arp2/3 regulators in actin assembly and endocytosis. Kirschner MW, editor. PLoS Biol. 2008;6:e1.

26. Mendoza MC. Phosphoregulation of the WAVE regulatory complex and signal integration. Semin Cell Dev Biol. Elsevier Ltd; 2013;24:272-9.

27. Pocha SM, Cory GO. WAVE2 is regulated by multiple phosphorylation events within its VCA domain. Cell Motil Cytoskeleton. 2009;66:36-47.

28. Cory GOC, Cramer R, Blanchoin L, Ridley AJ. Phosphorylation of the WASP-VCA domain increases its affinity for the Arp2/3 complex and enhances actin polymerization by WASP. Mol Cell. 2003;11:1229-39.

29. Galovic M, Xu D, Areces LB, van der Kammen R, Innocenti M. Interplay between NWASP and CK2 optimizes clathrin-mediated endocytosis of EGFR. J Cell Sci. 2011;124:2001-12.

30. Clark ES, Brown B, Whigham AS, Kochaishvili a, Yarbrough WG, Weaver a M. Aggressiveness of HNSCC tumors depends on expression levels of cortactin, a gene in the 11q13 amplicon. Oncogene. 2009;28:431-44.

31. Clark ES, Whigham AS, Yarbrough WG, Weaver AM. Cortactin is an essential regulator of matrix metalloproteinase secretion and extracellular matrix degradation in invadopodia. Cancer Res. 2007;67:4227-35.

32. Oser M, Yamaguchi H, Mader CC, Bravo-Cordero JJ, Arias M, Chen X, et al. Cortactin 
regulates cofilin and N-WASp activities to control the stages of invadopodium assembly and maturation. J Cell Biol. 2009;186:571-87.

33. Chuma M, Sakamoto M, Yasuda J, Fujii G, Nakanishi K, Tsuchiya A, et al. Overexpression of cortactin is involved in motility and metastasis of hepatocellular carcinoma. J Hepatol. 2004;41:629-36.

34. Zhang H-X, Jiang S-S, Zhang X-F, Zhou Z-Q, Pan Q-Z, Chen C-L, et al. Protein kinase CK2 $\alpha$ catalytic subunit is overexpressed and serves as an unfavorable prognostic marker in primary hepatocellular carcinoma. Oncotarget. 2015;

35. Ku MJ, Park JW, Ryu BJ, Son Y-J, Kim SH, Lee SY. CK2 inhibitor CX4945 induces sequential inactivation of proteins in the signaling pathways related with cell migration and suppresses metastasis of A549 human lung cancer cells. Bioorg Med Chem Lett. Elsevier Ltd; 2013;23:5609-13.

36. Benavent Acero F, Capobianco CS, Garona J, Cirigliano SM, Perera Y, Urtreger AJ, et al. CIGB-300, an anti-CK2 peptide, inhibits angiogenesis, tumor cell invasion and metastasis in lung cancer models. Lung Cancer. 2017;107:14-21.

37. Luo M-L, Shen X-M, Zhang Y, Wei F, Xu X, Cai Y, et al. Amplification and overexpression of CTTN (EMS1) contribute to the metastasis of esophageal squamous cell carcinoma by promoting cell migration and anoikis resistance. Cancer Res. 2006;66:11690-9.

38. Gibcus JH, Mastik MF, Menkema L, de Bock GH, Kluin PM, Schuuring E, et al. Cortactin expression predicts poor survival in laryngeal carcinoma. Br J Cancer. 2008;98:950-5.

39. Rodrigo JP, Garcı LA, Ramos S, Lazo PS, Sua C, García LA, et al. EMS1 Gene Amplification Correlates with Poor Prognosis in Squamous Cell Carcinomas of the Head and Neck. Clin cancer Res. 2000;6:3177-82.

40. Weaver AM. Cortactin in tumor invasiveness. Cancer Lett. 2008;265:157-66.

41. Dedes KJ, Lopez-Garcia M-A, Geyer FC, Lambros MBK, Savage K, Vatcheva R, et al. Cortactin gene amplification and expression in breast cancer: a chromogenic in situ hybridisation and immunohistochemical study. Breast Cancer Res Treat. 2010;124:65366.

42. Rodrigo JP, García-Carracedo D, García LA, Menéndez S, Allonca E, González M V, et al. Distinctive clinicopathological associations of amplification of the cortactin gene at $11 q 13$ in head and neck squamous cell carcinomas. J Pathol. 2009;217:516-23.

43. Yuan B-Z, Zhou X, Zimonjic DB, Durkin ME, Popescu NC. Amplification and Overexpression of the EMS 1 Oncogene, a Possible Prognostic Marker, in Human Hepatocellular Carcinoma. J Mol Diagnostics. 2003;5:48-53.

44. Rothschild BL, Shim AH, Ammer AG, Kelley LC, Irby KB, Head J a, et al. Cortactin overexpression regulates actin-related protein $2 / 3$ complex activity, motility, and invasion in carcinomas with chromosome 11q13 amplification. Cancer Res. 2006;66:8017-25.

45. Hui R, Campbell DH, Lee CS, McCaul K, Horsfall DJ, Musgrove EA, et al. EMS1 amplification can occur independently of CCND1 or INT-2 amplification at 11q13 and may identify different phenotypes in primary breast cancer. Oncogene. 1997;15:1617-23.

46. Chua MMJ, Ortega CE, Sheikh A, Lee M, Abdul-Rassoul H, Hartshorn KL, et al. CK2 in Cancer: Cellular and Biochemical Mechanisms and Potential Therapeutic Target. 
Pharmaceuticals (Basel). Multidisciplinary Digital Publishing Institute (MDPI); 2017;10.

47. Faust RA, Niehans G, Gapany M, Hoistad D, Knapp D, Cherwitz D, et al. Subcellular immunolocalization of protein kinase CK2 in normal and carcinoma cells. Int J Biochem Cell Biol. 1999;31:941-9.

48. Yenice S, Davis AT, Goueli SA, Akdas A, Limas C, Ahmed K. Nuclear casein kinase 2 (CK-2) activity in human normal, benign hyperplastic, and cancerous prostate. Prostate. 1994;24:11-6.

49. Trembley JH, Chen Z, Unger G, Slaton J, Kren BT, Van Waes C, et al. Emergence of protein kinase CK2 as a key target in cancer therapy. Biofactors. NIH Public Access; 2010;36:187-95.

50. Di Maira G, Brustolon F, Bertacchini J, Tosoni K, Marmiroli S, Pinna LA, et al. Pharmacological inhibition of protein kinase CK2 reverts the multidrug resistance phenotype of a CEM cell line characterized by high CK2 level. Oncogene. 2007;26:691526.

51. Landesman-Bollag E, Song DH, Romieu-Mourez R, Sussman DJ, Cardiff RD, Sonenshein GE, et al. Protein kinase CK2: signaling and tumorigenesis in the mammary gland. Mol Cell Biochem. 2001;227:153-65.

52. Kramerov AA, Saghizadeh M, Caballero S, Shaw LC, Li Calzi S, Bretner M, et al. Inhibition of protein kinase CK2 suppresses angiogenesis and hematopoietic stem cell recruitment to retinal neovascularization sites. Mol Cell Biochem. 2008;316:177-86.

53. Brown MS, Diallo OT, Hu M, Ehsanian R, Yang X, Arun P, et al. CK2 Modulation of NF- B, TP53, and the Malignant Phenotype in Head and Neck Cancer by Anti-CK2 Oligonucleotides In vitro or In vivo via Sub-50-nm Nanocapsules. Clin Cancer Res. 2010;16:2295-307.

54. Duncan JS, Litchfield DW. Too much of a good thing: the role of protein kinase CK2 in tumorigenesis and prospects for therapeutic inhibition of CK2. Biochim Biophys Acta. 2008;1784:33-47.

55. Ahmad KA, Wang G, Slaton J, Unger G, Ahmed K. Targeting CK2 for cancer therapy. Anticancer Drugs. 2005;16:1037-43.

56. Mishra S, Pertz V, Zhang B, Kaur P, Shimada H, Groffen J, et al. Treatment of P190 Bcr/Abl lymphoblastic leukemia cells with inhibitors of the serine/threonine kinase CK2. Leukemia. 2007;21:178-80.

57. Sandholt IS, Olsen BB, Guerra B, Issinger O-G. Resorufin: a lead for a new protein kinase CK2 inhibitor. Anticancer Drugs. 2009;20:238-48.

58. Yamane K, Kinsella TJ. CK2 inhibits apoptosis and changes its cellular localization following ionizing radiation. Cancer Res. 2005;65:4362-7.

59. Sivanand S, Peña-Llopis S, Zhao H, Kucejova B, Spence P, Pavia-Jimenez A, et al. A validated tumorgraft model reveals activity of dovitinib against renal cell carcinoma. Sci Transl Med. 2012;4:137ra75.

60. Das Thakur M, Salangsang F, Landman AS, Sellers WR, Pryer NK, Levesque MP, et al. Modelling vemurafenib resistance in melanoma reveals a strategy to forestall drug resistance. Nature. 2013;494:251-5. 
61. Merk J, Rolff J, Becker M, Leschber G, Fichtner I. Patient-derived xenografts of nonsmall-cell lung cancer: a pre-clinical model to evaluate adjuvant chemotherapy? Eur $\mathrm{J}$ Cardiothorac Surg. 2009;36:454-9.

62. Konotop G, Bausch E, Nagai T, Turchinovich A, Becker N, Benner A, et al. Pharmacological inhibition of centrosome clustering by slingshot-mediated cofilin activation and actin cortex destabilization. Cancer Res. 2016;

63. Liu L, Li J, Zhang L, Zhang F, Zhang R, Chen X, et al. Cofilin phosphorylation is elevated after F-actin disassembly induced by Rac1 depletion. Biofactors. 2015;41:352-9.

64. Cai L, Makhov AM, Schafer D a, Bear JE. Coronin 1B antagonizes cortactin and remodels Arp2/3-containing actin branches in lamellipodia. Cell. 2008;134:828-42.

65. Mandal T, Bhowmik A, Chatterjee A, Chatterjee U, Chatterjee S, Ghosh MK. Reduced phosphorylation of Stat3 at Ser-727 mediated by casein kinase 2 - Protein phosphatase $2 \mathrm{~A}$ enhances Stat3 Tyr-705 induced tumorigenic potential of glioma cells. Cell Signal. 2014;26:1725-34.

66. Hériché JK, Lebrin F, Rabilloud T, Leroy D, Chambaz EM, Goldberg Y. Regulation of protein phosphatase $2 \mathrm{~A}$ by direct interaction with casein kinase 2alpha. Science. 1997;276:952-5.

67. Sarrouilhe D, Filhol O, Leroy D, Bonello G, Baudry M, Chambaz EM, et al. The tight association of protein kinase CK2 with plasma membranes is mediated by a specific domain of its regulatory beta-subunit. Biochim Biophys Acta. 1998;1403:199-210.

68. Wei T, Tao M. Human erythrocyte casein kinase II: characterization and phosphorylation of membrane cytoskeletal proteins. Arch Biochem Biophys. 1993;307:206-16.

69. van Weerden WM, de Ridder CM, Verdaasdonk CL, Romijn JC, van der Kwast TH, Schröder FH, et al. Development of seven new human prostate tumor xenograft models and their histopathological characterization. Am J Pathol. 1996;149:1055-62.

70. Reyal F, Guyader C, Decraene C, Lucchesi C, Auger N, Assayag F, et al. Molecular profiling of patient-derived breast cancer xenografts. Breast Cancer Res. 2012;14:R11.

71. Tentler JJ, Tan AC, Weekes CD, Jimeno A, Leong S, Pitts TM, et al. Patient-derived tumour xenografts as models for oncology drug development. Nat Rev Clin Oncol. 2012;9:338-50.

72. Jung J, Lee CH, Seol HS, Choi YS, Kim E, Lee EJ, et al. Generation and molecular characterization of pancreatic cancer patient-derived xenografts reveals their heterologous nature. Oncotarget. 2016;7:62533-46.

73. Basu D, Nguyen T-TK, Montone KT, Zhang G, Wang L-P, Diehl JA, et al. Evidence for mesenchymal-like sub-populations within squamous cell carcinomas possessing chemoresistance and phenotypic plasticity. Oncogene. 2010;29:4170-82.

74. Alizadeh AA, Aranda V, Bardelli A, Blanpain C, Bock C, Borowski C, et al. Toward understanding and exploiting tumor heterogeneity. Nat Med. 2015;21:846-53.

75. Tabassum DP, Polyak K. Tumorigenesis: It takes a village. Nat Rev Cancer. Nature Publishing Group; 2015;15:473-83.

76. De Wever O, Mareel M. Role of tissue stroma in cancer cell invasion. J Pathol. 
2003;200:429-47.

77. Gengenbacher N, Singhal M, Augustin HG. Preclinical mouse solid tumour models: status quo, challenges and perspectives. Nat Rev Cancer. Nature Publishing Group; 2017; 17:751-65.

78. Day C-P, Merlino G, Van Dyke T. Preclinical mouse cancer models: a maze of opportunities and challenges. Cell. NIH Public Access; 2015;163:39-53.

79. Migliardi G, Sassi F, Torti D, Galimi F, Zanella ER, Buscarino M, et al. Inhibition of MEK and $\mathrm{PI} 3 \mathrm{~K} / \mathrm{mTOR}$ suppresses tumor growth but does not cause tumor regression in patient-derived xenografts of RAS-mutant colorectal carcinomas. Clin Cancer Res. 2012;18:2515-25.

80. Keysar SB, Astling DP, Anderson RT, Vogler BW, Bowles DW, Morton JJ, et al. A patient tumor transplant model of squamous cell cancer identifies PI3K inhibitors as candidate therapeutics in defined molecular bins. Mol Oncol. 2013;7:776-90.

81. Cancer in North America: 2011-2015. 2018.

82. Wilson RJ, Ryerson AB, Singh SD, King JB. Cancer Incidence in Appalachia, 2004-2011. Cancer Epidemiol Biomarkers Prev. 2016;25:250-8.

83. 2017 West Virginia Cancer Burden Report. Morgantown; 2017.

84. Vermorken JB, Mesia R, Rivera F, Remenar E, Kawecki A, Rottey S, et al. Platinumbased chemotherapy plus cetuximab in head and neck cancer. $\mathrm{N}$ Engl J Med. 2008;359:1116-27. 


\section{APPENDIX}




\title{
Video Article \\ Quantitative Measurement of Invadopodia-mediated Extracellular Matrix Proteolysis in Single and Multicellular Contexts
}

\author{
Karen H. Martin, Karen E. Hayes, Elyse L. Walk, Amanda Gatesman Ammer, Steven M. Markwell, Scott A. Weed \\ Department of Neurobiology and Anatomy, Program in Cancer Cell Biology, Mary Babb Randolph Cancer Center, West Virginia University
}

Correspondence to: Scott A. Weed at scweed@hsc.wvu.edu

URL: http://www.jove.com/video/4119/

DOI: $10.3791 / 4119$

Keywords: Cellular Biology, Issue 66, Cancer Biology, Anatomy, Molecular Biology, Biochemistry, invadopodia, extracellular matrix, gelatin, confocal microscopy, quantification, oregon green

Date Published: $8 / 27 / 2012$

Citation: Martin, K.H., Hayes, K.E., Walk, E.L., Ammer, A.G., Markwell, S.M., Weed, S.A. Quantitative Measurement of Invadopodia-mediated Extracellular Matrix Proteolysis in Single and Multicellular Contexts. J. Vis. Exp. (66), e4119 10.3791/4119, DOI : 10.3791/4119 (2012).

\section{Abstract}

Cellular invasion into local tissues is a process important in development and homeostasis. Malregulated invasion and subsequent cell movement is characteristic of multiple pathological processes, including inflammation, cardiovascular disease and tumor cell metastasis ${ }^{1}$. Focalized proteolytic degradation of extracellular matrix (ECM) components in the epithelial or endothelial basement membrane is a critical step in initiating cellular invasion. In tumor cells, extensive in vitro analysis has determined that ECM degradation is accomplished by ventral actin-rich membrane protrusive structures termed invadopodia ${ }^{2,3}$. Invadopodia form in close apposition to the ECM, where they moderate ECM breakdown through the action of matrix metalloproteinases (MMPs). The ability of tumor cells to form invadopodia directly correlates with the ability to invade into local stroma and associated vascular components ${ }^{3}$.

Visualization of invadopodia-mediated ECM degradation of cells by fluorescent microscopy using dye-labeled matrix proteins coated onto glass coverslips has emerged as the most prevalent technique for evaluating the degree of matrix proteolysis and cellular invasive potential ${ }^{4,5}$. Here we describe a version of the standard method for generating fluorescently-labeled glass coverslips utilizing a commercially available Oregon Green-488 gelatin conjugate. This method is easily scaled to rapidly produce large numbers of coated coverslips. We show some of the common microscopic artifacts that are often encountered during this procedure and how these can be avoided. Finally, we describe standardized methods using readily available computer software to allow quantification of labeled gelatin matrix degradation mediated by individual cells and by entire cellular populations. The described procedures provide the ability to accurately and reproducibly monitor invadopodia activity, and can also serve as a platform for evaluating the efficacy of modulating protein expression or testing of anti-invasive compounds on extracellular matrix degradation in single and multicellular settings.

\section{Video Link}

The video component of this article can be found at http://www.jove.com/video/4119/

\section{Protocol}

\section{Production of Oregon Green 488-gelatin Coated Coverslips}

1. Prepare an unlabeled $5 \%(\mathrm{w} / \mathrm{w})$ stock gelatin/sucrose solution by adding $1.25 \mathrm{~g}$ gelatin and $1.25 \mathrm{~g}$ sucrose in PBS to a final volume of $50 \mathrm{ml}$. Warm the stock gelatin solution to $37^{\circ} \mathrm{C}$ and ensure it is entirely melted before use. Store the final mixture at $4{ }^{\circ} \mathrm{C}$.

2. Clean $13 \mathrm{~mm}$ diameter \#1 glass coverslips by placing an individual coverslip into each well of a 24 well plastic tissue culture plate. Add $500 \mu \mathrm{l}$ of $20 \%$ nitric acid to each well and incubate for $30 \mathrm{~min}$. Aspirate the nitric acid solution and wash coverslips three times with deionized water.

3. Coat coverslips with $500 \mu \mathrm{l}$ of $50 \mu \mathrm{g} / \mathrm{ml}$ poly-L-lysine (prepared from $0.1 \%$ stock solution and diluted in deionized water) to each well for 20 min at room temperature. Aspirate the solution and wash three times with PBS. Poly-L-lysine coating facilitates even coating and bonding of the overlying labeled gelatin.

4. Add $500 \mu \mathrm{l}$ of $0.5 \%$ glutaraldehyde (made fresh before use) to each well and incubate the 24 well plates on ice for 15 min. Aspirate and wash three times with cold PBS. Be sure to remove all traces of PBS prior to gelatin coating. Keep plates on ice during all washes until gelatin is added.

5. Reconstitute the Oregon Green 488-conjugated gelatin as per manufacturer's protocol and warm it and the unlabeled $5 \%$ gelatin/sucrose solution from (1.1) to $37^{\circ} \mathrm{C}$. Dilute one part Oregon Green 488 gelatin into eight parts of unlabeled gelatin/sucrose (i.e.; $500 \mu \mathrm{l}$ of Oregon Green 488 gelatin into $4 \mathrm{ml}$ of $5 \%$ gelatin mixture). Pipet $100 \mu \mathrm{l}$ of the diluted 488 -gelatin mixture (kept at $37^{\circ} \mathrm{C}$ ) onto each coverslip, using enough gelatin to coat the coverslip without manual spreading (which can lead to uneven coverslip coating as shown in Figure 3B). It is important to keep the diluted 488 -gelatin mixture at $37^{\circ} \mathrm{C}$ during the coating procedure to prevent premature solidification. From this step forward the coverslips should be kept in the dark as much as possible to avoid potential photobleaching. Other ECM proteins conjugated to different fluorophores can be substituted for Oregon Green 488 gelatin (see Discussion). 
6. Once all coverslips are coated in a single plate, hold the 24 well plate at an angle and remove excess gelatin from each well by vacuum aspiration. Incubate coated coverslips in the dark for $10 \mathrm{~min}$ at room temperature.

7. Wash the coverslips three times with PBS, then add $500 \mu \mathrm{l}$ of freshly made $5 \mathrm{mg} / \mathrm{ml}$ sodium borohydride $\left(\mathrm{NaBH}_{4}\right)$ for $15 \mathrm{~min}$ at room temperature to reduce and inactivate residual glutaraldehyde. Sodium borohydride is effervescent, and small bubbles will be evident on and around each coverslip.

8. Remove the $\mathrm{NaBH}_{4}$ solution by vacuum aspiration with a quick sweeping motion around the outside of each well. Take care not to pick up any floating coverslips that became detached from the bottom of the tissue culture plate during $\mathrm{NaBH}_{4}$ treatment. Detached coverslips that float to the top may be gently pushed back down to the well bottom, but care must be taken to avoid damaging the protein coating. Wash each well three times with PBS and then incubate coverslips in 70\% ethanol for $30 \mathrm{~min}$ at room temperature.

9. Using sterile technique, transfer the coverslip-containing plates to a type IIA/B cell culture laminar flow hood and rinse coverslips three times with sterile PBS. At this point coverslips can be stored in PBS protected from light at $4{ }^{\circ} \mathrm{C}$ for at least two months.

10. Transfer coverslips to be used for degradation assays to an empty well of a new 24 well plate by careful removal using a sterile needle and forceps. Equilibrate coverslips for 1-24 hr with complete media appropriate to the specific cell type being assayed. Care must be taken not to invert the coverslip or scratch the gelatin coating (see Figure 3B).

\section{Plating and Processing of Cells on Oregon Green 488-gelatin Coated Coverslips to Assay ECM Degradation}

1. Seed $3-5 \times 10^{4}$ cells onto a coverslip within each well of the 24 well plate.

2. Conduct a time course study to determine optimal times required for invadopodia degradation activity for the particular cell line/type of interest. Most invasive cells require a time between $4-24 \mathrm{~h}$ for degradation to become apparent, although this range can vary widely and should be empirically determined. To synchronize invadopodia activity, cells can be treated with MMP inhibitors (e.g., GM 6001) for a desired time period, then wash out the inhibitor to allow invadopodia activity to proceed (for example, see ${ }^{6}$ ).

3. Rinse coverslips three times with PBS, then fix cells with $500 \mu \mathrm{l}$ of $10 \%$ buffered formalin phosphate for 15 min. Rinse three times with PBS and permeabilize for 4 min with $0.4 \%$ Triton X-100 in PBS. Rinse three times with PBS to remove the Triton X-100.

4. Label cells using any standard protocol for immunofluorescence staining (see ${ }^{7}$ for example) by co-labeling cells with fluorescent conjugated phalloidin to visualize actin filaments (F-actin) and for a known marker protein that localizes to invadopodia (e.g; $\operatorname{cortactin}^{5}$, TKS5 ${ }^{8}$, or NWASp ${ }^{9}$ ). Remember to avoid using 488-labeled secondary antibodies or GFP-labeled proteins if using Oregon Green 488 or FITC-labeled gelatin to prevent signal interference.

5. Mount stained coverslips onto glass microscope slides by carefully inverting the coverslip and placing it on a drop of ProLong Gold antifade or similar reagent.

6. To assess matrix degradation, image cells in appropriate channels using a conventional fluorescent or confocal microscope. Gelatin degradation is visualized as darker areas on the coverslip due to proteolytic removal of the fluorescent gelatin (Figure 4A). Labeling of cells for actin and an invadopodia marker protein allows for confirmation of invadopodia at sites of matrix degradation in merged images (Figure 4A).

7. Degradation activity can also be monitored in real time by live cell imaging with fluorescent-tagged recombinant proteins to track invadopodia formation and matrix degradation ${ }^{5,10,11}$.

\section{Quantification of Fluorescent Gelatin Degradation by Measuring Normalized Matrix Degradation}

This analysis provides the normalized area of matrix degradation relative to the area of the cells or the number of cells. It is useful for analyzing entire microscopic fields of view where multiple cells are present that have been collectively treated with siRNA, growth factors or therapeutic agents. For this analysis, images collected at lower magnification are sufficient to efficiently collect information about populations of cells.

1. Open the images in Image $\mathrm{J}^{12}$. ImageJ for microscopy can be downloaded from http://www.macbiophotonics.ca/imagej/.

2. Check the scale information by choosing the menu command "Analyze/Set Scale." This information will import automatically with many file formats, but can be entered manually if required. Proper scaling is necessary to report measurements in microns rather than pixels.

3. Select the appropriate measurements to track by choosing "Analyze/Set Measurements." Check Area and Limit to Threshold.

4. Calculate the area of degradation using the fluorescent gelatin image (Figure 5A).

5. Threshold the image ("Image/Adjust/Threshold") to set the upper and lower pixel intensity values to select the areas of degradation (highlighted in red; Figure 5B). In subsequent images, use the Set button in the Threshold window to set the same threshold for all images as an objective means to select degradation area.

6. In some cases, the coverslip may not be perfectly flat when images are acquired. This causes the intensity of the gelatin to change across the image. If this variation creates problems when thresholding the image, correct for uneven illumination across the gelatin by subtracting the background ("Process/Subtract Background") or by filtering with a bandpass filter ("Process/FFT/Bandpass Filter") or a pseudo flatfield filter ("Process/Filters/Pseudo Flatfield") until the background intensity is uniform.

7. Measure the area of matrix degradation ("Analyze/Analyze Particles"). In the Analyze Particles window, choose a particle size $>0$ to remove noise from the selection. Show Outlines to identify regions of interest (ROIs). Check Display Results and Summarize to show measurements. If the drawing has specifically outlined all of the areas of degradation (Figure 5C), copy the Total Area measurement into a spreadsheet. If other objects were selected (such as debris), record only the areas of the relevant ROls.

8. Calculate the cell area using the phalloidin stained (F-actin) image (Figure 5D).

9. Threshold the image ("Image/Adjust/Threshold") to set the upper and lower pixel intensity values so that the edges of the cells are selected (highlighted in red; Figure 5E). In subsequent images, use the Set button in the Threshold window to set the same threshold for all images as an objective means to select cell area. 
10. 10 Measure the area of the cells ("Analyze/Analyze Particles"). In the Analyze Particles window, choose a particle size $>0$ to remove noise from the selection. Show Outlines to identify regions for analysis (Figure 5F). Check Display Results and Summarize to show area measurements. Do not check Include Holes if there are spaces between cells in a cluster so the non-selected pixels within the cluster will not be included in the cell area calculation. Choose OK.

11. Copy the Area results for relevant ROls into a spreadsheet.

12. Calculate the area of gelatin degradation per total area of cells ${ }^{13}$

13. An alternative approach would be to report the area of degradation per number of cells from counting nuclei (Figure $\mathbf{5 G}$ ). This is necessary if manipulations alter the cell area between different compared treatment groups. Automatic counting works best if nuclei are well separated uniform in intensity and round. Automatically count nuclei ("Plugins/Particle Analysis/Nucleus Counter"). Choose Smallest and Largest Particle Size, a Threshold Method and a Smoothing Method. Check Subtract Background, Watershed Filter, Add Particles to ROI Manager and Show Summary (Figure 5H).

14. If nuclei overlap extensively or have an irregular shape or texture, automatic counting may not produce an accurate count (Figure $\mathbf{5 H}$, arrows on right). In this case, manual counting can be facilitated using the cell counter tool ("Plugins/Particle Analysis/Cell Counter"). This will keep count as cells are marked during a manual count (Figure 5I).

15. Copy the number of cells (nuclei) into a spreadsheet. Calculate the area of gelatin degradation per total number of cells.

\section{Quantification of Fluorescent Gelatin Degradation by Individual Cells in a Mixed Cellular Population}

To evaluate matrix degradation resulting from specific cells in a population apart from other cells within the field (e.g., transfected versus nontransfected cells), the procedure in section 3 can be modified to measure the area of degradation under individual cells. An additional fluorescent channel is needed to mark transfected cells. In this instance, higher magnification images and well-separated cells are easier to quantitate.

1. Check the scale information by choosing the menu command "Analyze/Set Scale." Select the appropriate measurements to track by choosing "Analyze/Set Measurements." Check Area and Limit to Threshold.

2. For individual cells that are not touching, identify each cell using the F-actin image (Figure 6A). Threshold the image (see 3.9) (Figure 6B). It is important to capture the edges of the cells, but there can be holes inside that are not included in the threshold. Use the same intensity values across images to select cell boundaries.

3. To measure the area of the cells, use "Analyze/Analyze Particles." In the Analyze Particles window, choose a Size $>0$ (to eliminate noise), Show Outlines, and check Display Results, Add to Manager and Include Holes (to record the entire area inside the outline). Choose OK and record the Area for each cell from the Results window.

4. Identify which cells are transfected (Figure 6C).

5. Identify the areas of degradation using the fluorescent gelatin image (Figure 6D). If needed, filter the gelatin image to even background intensity (see 3.6). Threshold to select the areas of degradation, making note of the threshold settings (Figure 6E). On subsequent images, use these same upper and lower intensity values (using the Set button in the Threshold window) for an objective selection of areas of degradation.

6. Measure the areas of degradation under the cells. On the thresholded fluorescent gelatin image, show an outline of the cells by selecting ROIs in the ROI Manager window and selecting Measure (Figure 6F). Record the results and calculate the normalized area of degradation/ cell or cell area.

\section{Representative Results}

The overall schematic for the procedure is shown in Figure 1. The procedure entails preparation of glass coverslips and coating with fluorescently-conjugated gelatin, plating of cells onto the coated coverslips to allow cells to degrade the gelatin, fixing and labeling of cells for fluorescence microscopic analysis, imaging the fluorescent matrix to assess the matrix integrity, and objectively quantifying the degree of gelatin matrix degradation using computer software. 


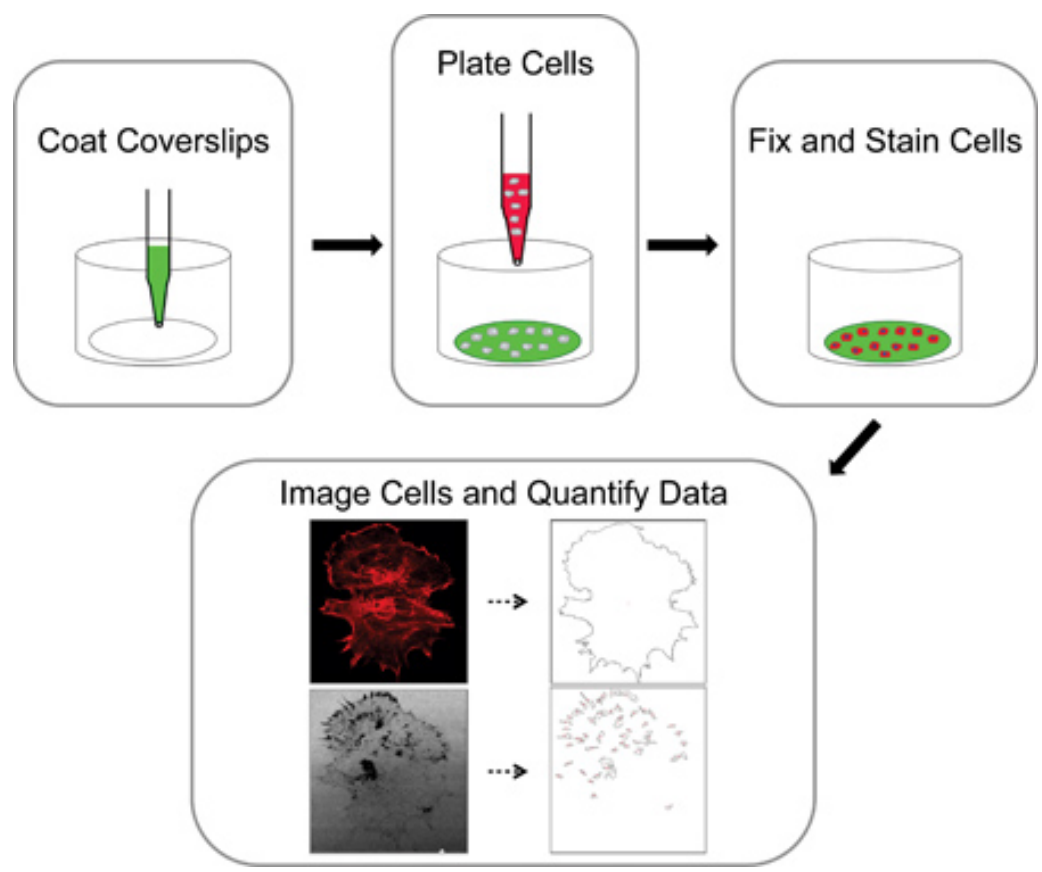

Figure 1. Overall schematic highlighting the key steps involved in fluorescent gelatin coating, cell plating, fixing and immunolabeling, and evaluating matrix proteolysis.

The key procedural steps involved in preparing and coating glass coverslips are outlined in Figure 2.
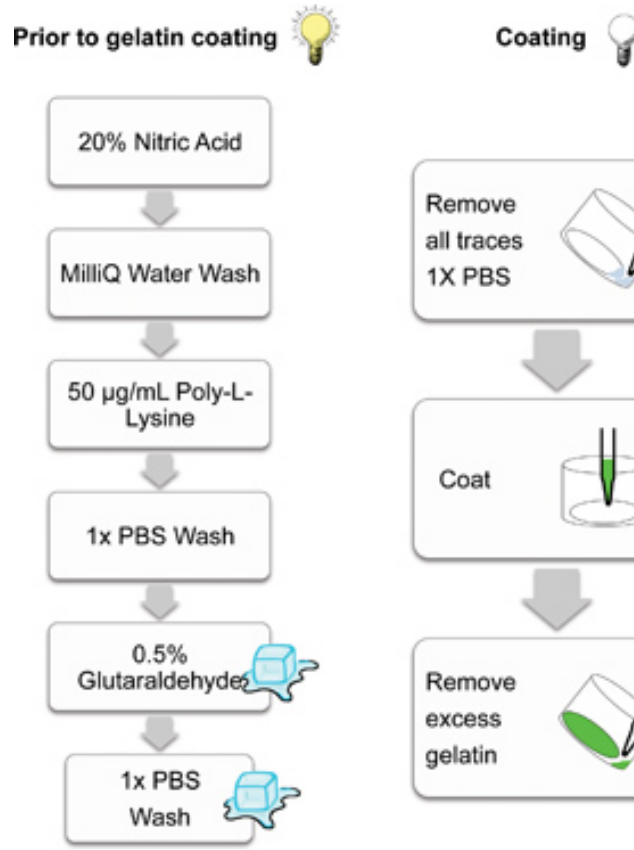

After Coating
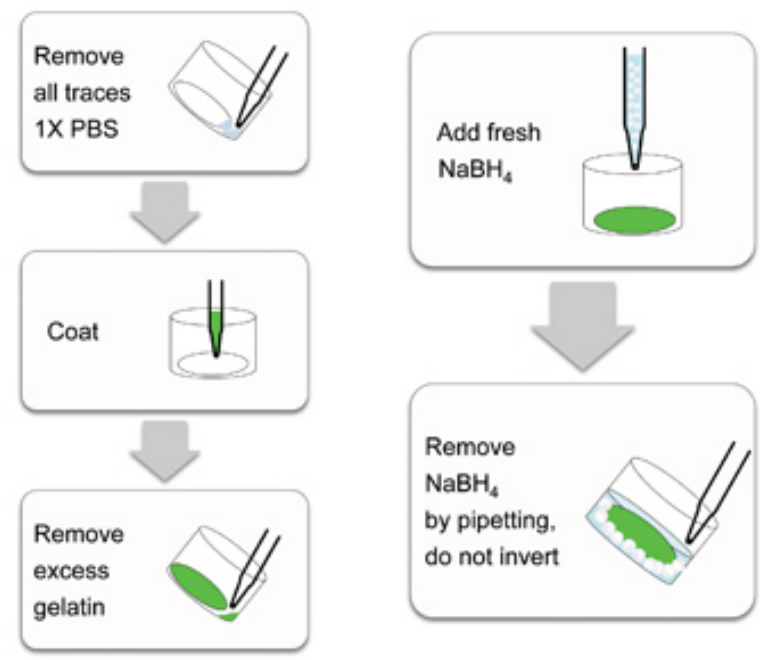

Figure 2. Schematic demonstrating the individual steps involved in preparing glass coverslips for gelatin matrix coating. Steps conducted in the light (lit bulb), on ice (cubes) and in the dark (non-illuminated bulb) are cartoon indicated. Steps conducted in the dark help prevent photobleaching of the fluorescent matrices.

When properly performed, coverslips are evenly coated with Oregon Green 488-conjugated gelatin, displaying homogenous fluorescence when visualized by microscopy (Figure 3A). Typical artifacts that can arise due to improper coating, handling, storage and usage of coated coverslips are shown in Figure 3B. 
A

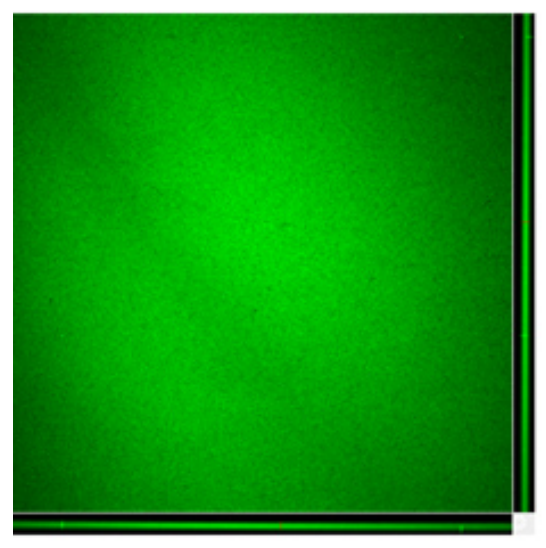

B
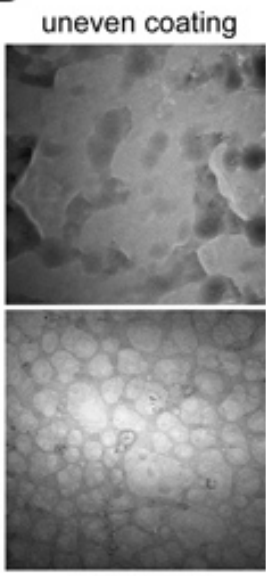

dehydrated

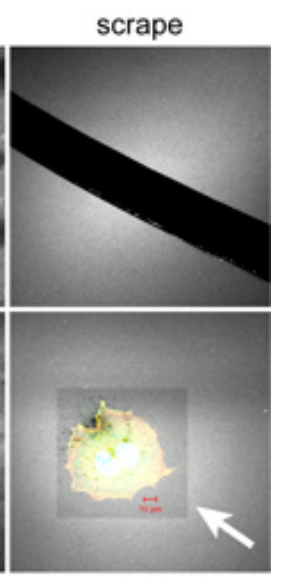

bleaching

Figure 3. Examples of artifacts encountered during gelatin coated coverslip preparation and handling. A. Orthogonal view of a confocal z-stack showing the typical color and consistency of an Oregon Green 488-conjugated gelatin coated coverslip produced using the prescribed protocol Coverslips should have a homogenous coating $\sim 1-2 \mu \mathrm{m}$ thick as shown in the X-Z (bottom) and Y-Z (right) confocal planes. B. Artifacts that can occur during the coating and processing of gelatin-coated coverslips include: Improper covering of the coverslip during the coating process due to poor mixing, manual spreading or partial solidification of the gelatin mixture (uneven coating), removal of the coated matrix by scoring with needles or forceps during handling (scrape), drying of the coverslip surface during prolonged storage periods, resulting in a "cobblestone" appearance (dehydrated) and photobleaching of the fluorescent gelatin surface during imaging due to prolonged or high intensity light exposure (bleaching). White arrow indicates bleached area encompassing a plated OSC19 head and neck squamous carcinoma cell. The Oregon Green 488-conjugated gelatin is pseudocolored white to enhance image contrast. Bar, $10 \mu \mathrm{m}$.

The resulting thin matrices produced during this procedure provide a sensitive means to evaluate the ability of cells to degrade ECM. Figure 4 demonstrates an example of invadopodia activity from an OSC19 cell plated on an Oregon Green-488 conjugated gelatin coverslip and imaged by conventional confocal microscopy as well as by volume-fill image rendering following three dimensional deconvolution. 

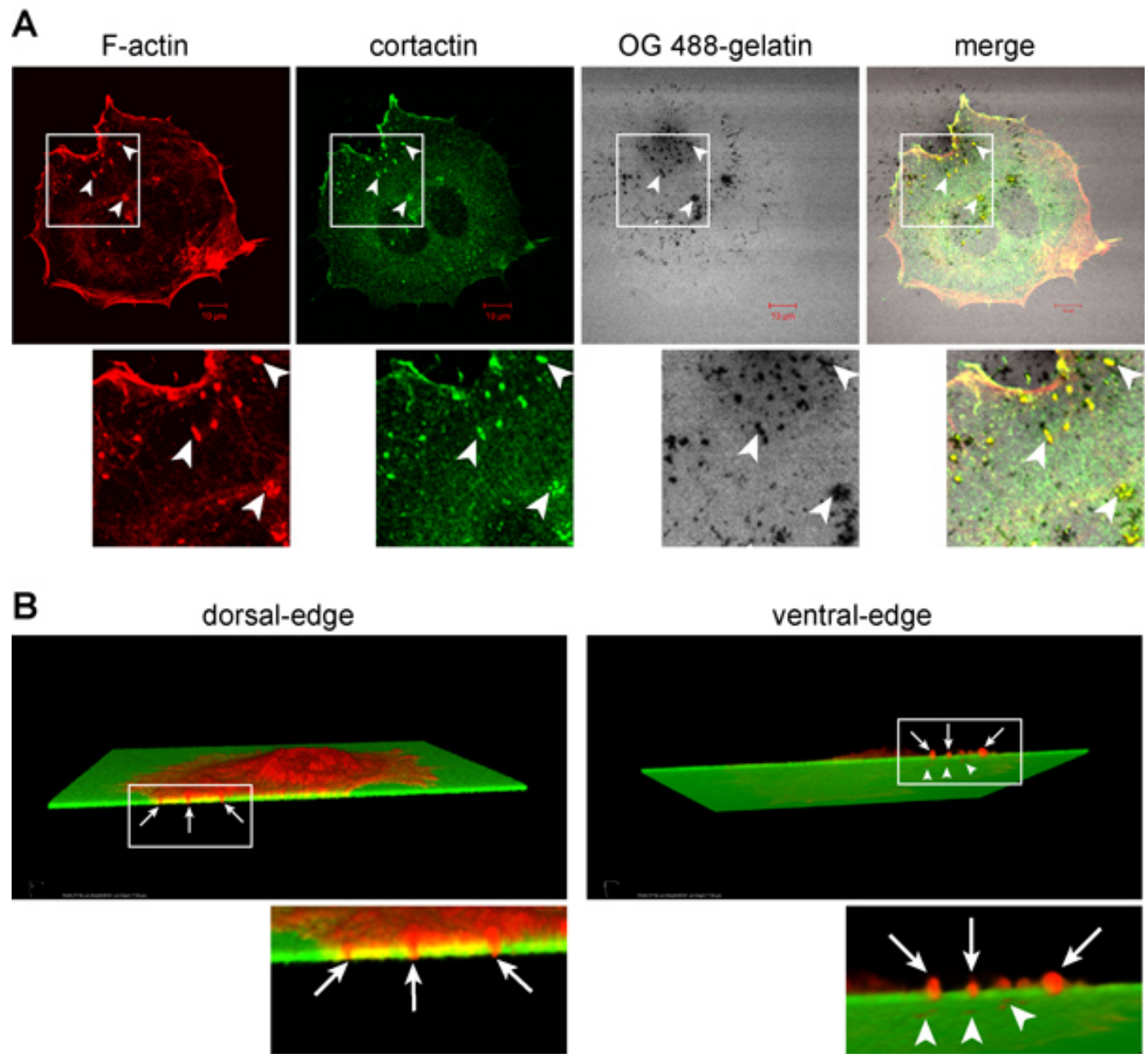

Figure 4. Representative examples of invadopodia matrix degradation activity. A. Visualization of invadopodia and corresponding gelatin matrix proteolysis. OSC19 cells plated on Oregon Green 488-conjugated gelatin coverslips for $10 \mathrm{hr}$ were fixed and labeled with rhodamine-conjugated phalloidin (F-actin) and anti-cortactin antibodies (visualized with an Alexa Fluor 647 secondary antibody and pseudocolored green). Invadopodia are evident as focal cytoplasmic concentrations of F-actin and cortactin that overlap with areas of gelatin clearing (dark holes in the matrix) within the merged image. Boxed regions containing arrowheads indicate individual invadopodia and areas of focal matrix proteolysis as shown in the enlarged regions below. Bar, $10 \mu \mathrm{m}$. B. Volume fill visualization of invadopodia penetration into the ECM. OSC19 cells plated and stained as in (A) were visually rendered by obtaining 23 successive $0.32 \mu \mathrm{m}$ optical z-slices totaling $7.04 \mu \mathrm{m}$ for rhodamine-conjugated phalloidin and Oregon Green 488-conjugated gelatin. The native LSM file set for each channel was opened in AutoQuant X2.2 software and a 3D blind deconvolution of each image stack was performed using the recommended settings (10 iterations, medium noise). The processed images were saved as TIFF stacks that were then opened in NIS Elements and rendered as a volume view with alpha blending. The LUTs were adjusted, and a subvolume was created to show an edge inside the cell where invadopodia are present. Dorsal-edge view demonstrates invadopodia (red, arrows) inserted into the underlying gelatin (green). Ventral-edge view shows protrusive invadopodia and areas of gelatin degradation underneath the coverslip as regions of red present in the green matrix (arrowheads). The total image field presented is cropped to $77 \times 65 \mu \mathrm{m}$; the cell is $60 \times 40 \mu \mathrm{m}$.

Figure 5 shows some of the important steps for quantification of normalized gelatin matrix degradation as described in step 3 of the protocol. This procedure is designed to allow for unbiased quantitation of gelatin degradation in an entire field of view, and is suitable for matrix degradation attributed to many cells within the field. 

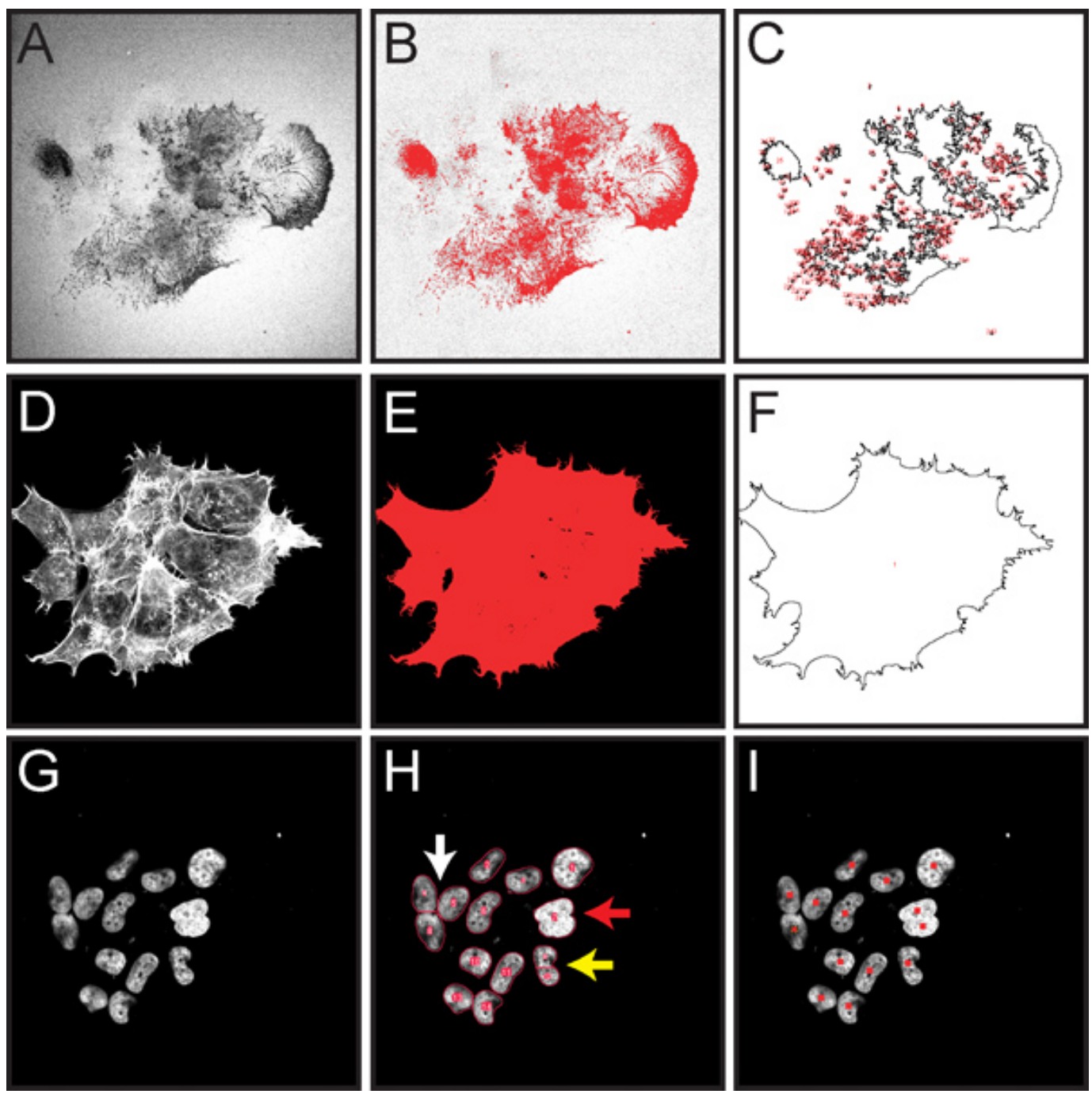

Figure 5. Screen capture images demonstrating key steps in computational-assisted quantification of normalized gelatin degradation for cells within an entire microscopic image as described in protocol step 3. All fluorescent images have been converted to grayscale to better display the red thresholding and ROI markings. A. Image of Oregon Green 488-conjugated gelatin, showing dark areas ("holes") where degradation has occurred (step 3.4). B. Thresholded gelatin image highlighting areas of degradation in red (step 3.5). C. Drawing showing ROls measured for area of degradation (step 3.7). D. Rhodamine phalloidin staining of F-actin (step 3.8). E. Thresholded actin image highlighting total cell area in red (step 3.9). F. Drawing showing cell areas to be measured (step 3.10). G. Image of DAPI-stained cell nuclei (step 3.13). H. Red outlines show results from automatic nuclei counting (step 3.13). The Watershed filter has the potential to separate nuclei that are touching (white arrow). If nuclei overlap extensively, they may not be separated into individual objects (red arrow). If a nucleus has an irregular shape, it may be separated into multiple objects (yellow arrow). I. Results from marking nuclei during a manual count using the cell counter tool (step 3.14).

Figure 6. demonstrates select steps involved in quantifying fluorescent gelatin degradation by individual cells within a mixed cellular population as described in protocol step 4. Here, matrix degradation by transfected cells can be analyzed within a mixed population of transfected and nontransfected cells. 

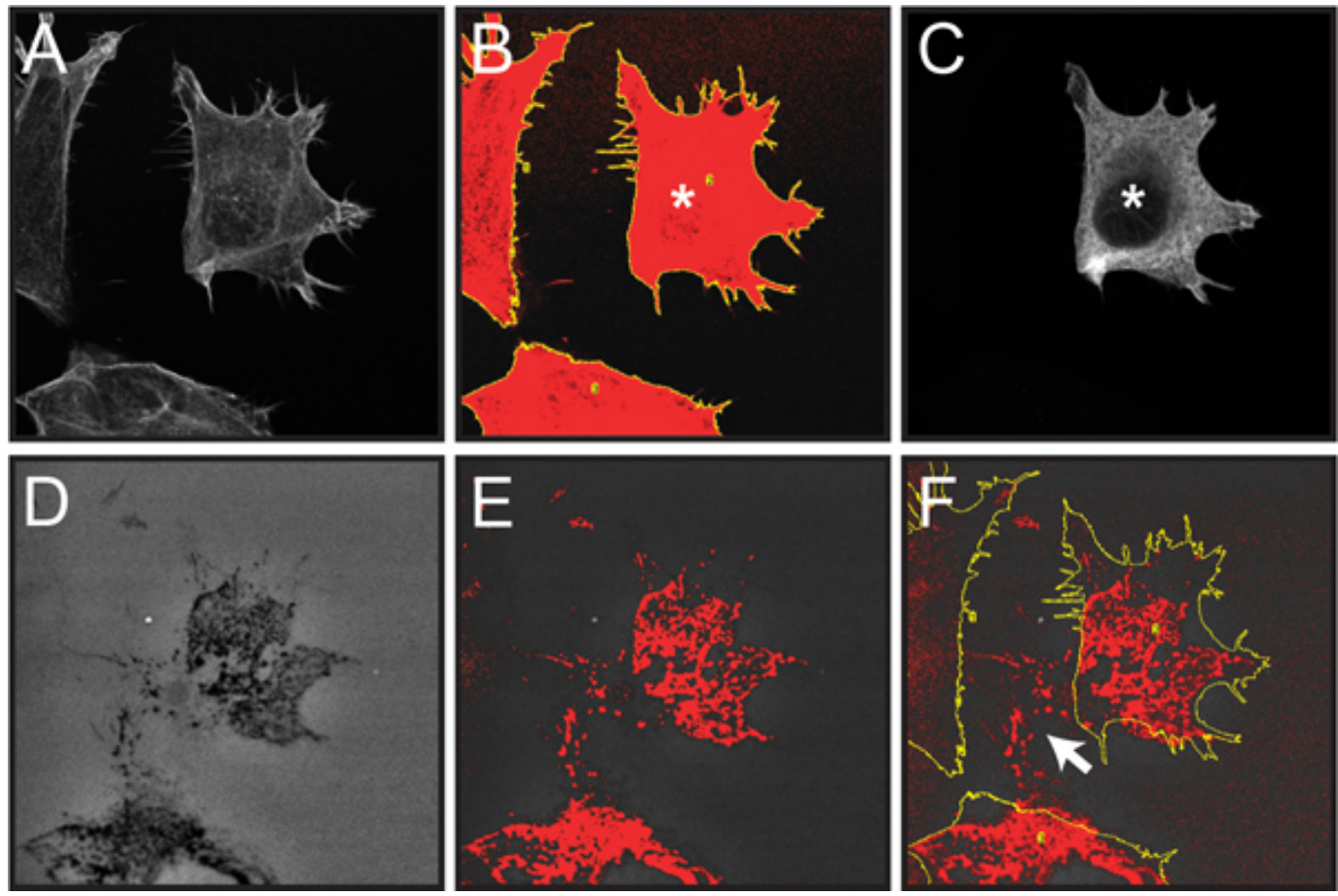

Figure 6. Screen capture images of steps involved in quantifying gelatin degradation from individual transfected cells within a cell population. Quantification of a single transfected OSC19 cell overexpressing recombinant cortactin fused to the FLAG epitope tag is shown as an example. All fluorescent images have been converted to grayscale to better display the red thresholding and yellow ROI markings. A. Confocal image of three cells labeled with rhodamine-phalloidin (step 4.2). B. Drawing of total cell area based on F-actin staining following application of the Threshold and Analyze Particles functions (step 4.2-3). C. Confocal image of anti-FLAG immunolabeling of the cell population demonstrating a single cell expressing FLAG-tagged cortactin (marked with *) (step 4.4). D. Image of Oregon Green 488-conjugated gelatin, showing dark areas ("holes") where degradation has occurred (step 4.5) E. Thresholded gelatin image highlighting dark areas of degradation in red (step 4.5). F. Thresholded gelatin image overlaid with cell outlines from panel B (step 4.6). Note that only the thresholded pixels within the cell outlines are counted in the analysis. Areas of degradation outside the current cell location (white arrow) result from cell migration across the gelatin over time and are not included in the analysis.

\section{Discussion}

The ability to visualize cells degrading the extracellular matrix has aided in discovering the molecular mechanisms employed in the early steps of cell invasion. Pioneered by Wen-Tien Chen in the early $1980^{\prime} \mathrm{s}^{4,14,15}$, coating fluorescently labeled extracellular proteins on glass coverslips for subsequent microscopic analysis has emerged as the primary technique in evaluating invadopodia function across a wide range of cell types. The prescribed protocol demonstrates the basic method used for preparing gelatin-coated coverslips that form a collagenous layer less than $2 \mu \mathrm{m}$ thick suitable for detection of extracellular matrix degradation by cells in most conventional fluorescent and confocal microscopes ${ }^{11,16-18}$, similar to what has been previously described ${ }^{19-21}$. These properties allow for rapid production of coated coverslips capable of detecting the initial onset of matrix degradation. The sensitivity afforded by the resulting thin gelatin matrix on the underlying hard glass surface likely aids in promoting invadopodia formation as a response to the high inherent stiffness of the overall matrix environment ${ }^{22}$. However, these matrices are not well suited for analysis of invadopodia elongation or additional morphological evaluation that has been achieved using thicker $(30-100 \mu \mathrm{m})$ gelatin layers with similar methodology, coated transwells or electron microscopy ${ }^{20,23,24}$

We have found that pre-conjugated commercially produced Oregon Green 488 gelatin allows for rapid experimental set up and consistent, reproducible results. However, alkaline borate conjugation of fluorescein isothiocyanate (FITC) to unlabeled gelatin remains a popular and inexpensive method for producing fluorescent gelatin conjugate ${ }^{20}$. Fibronectin is also used as an alternative matrix protein for labeling and coverslip coating ${ }^{4,9}$, and in some cases investigators have used labeled fibronectin layered onto unlabeled gelatin coated coverslips to create denser matrices ${ }^{11,25}$. Other matrices could be used, depending on the specifics of the cell type. In addition to dyes in the green $488 \mathrm{~nm}$ spectrum, a wide range of fluorophores have also been used with manual coupling methods to generate coverslips with different fluorescence spectra, including rhodamine ${ }^{21,26}$, Alexa Fluor $350^{24}, 546^{21}, 568^{5,11}, 594^{27}$ and $647^{5}$ dyes. Such conjugates are easily adaptable for use in the prescribed protocol, providing the flexibility for utilizing specific ECM protein-dye combinations suitable for most any imaging filter set.

The techniques described herein provide the necessary detailed steps for utilizing ImageJ to quantify gelatin matrix degradation attributed to individual cells in a heterogeneous population or to entire cell groups as published previously ${ }^{6,28}$. Proprietary software has also been successfully employed for the same purpose ${ }^{5,25}$. In this protocol, the area of matrix degradation is normalized to either the total area of the cells or the total number of cells (nuclei) in the field. Generally, both options for normalization will give the same result (ELW, data not shown). However, if different cell lines having different sized cells are being compared or if the experimental treatment causes cells to change size, then it may be 
more accurate to normalize to cell number. On the other hand, many cancer cell lines have a high percentage of multi-nucleated cells, in which case total cell area may be a more accurate parameter for normalization. Also, if only part of a cell is captured in an image (Figure 6), it may be better to normalize to cell area rather than underestimate the degradation potential for an individual cell. It is important to optimize the image analysis to best suit the characteristics and nuances of the specific experimental setup.

For determining cell numbers in a crowded field, counting nuclei is often the method of choice. ImageJ has a nucleus counter plugin for automatic counting. One option in this tool is the Watershed filter. This filter will help separate nuclei that are touching by segregating them into individual objects (Figure $\mathbf{5 H}$, white arrow). However, this filter may not be able to separate nuclei that overlap extensively (Figure $\mathbf{5} \mathbf{H}$, red arrow). In addition, if a nucleus has an irregular shape and large variations in intensity, the filter may separate a single nucleus into multiple objects (Figure $\mathbf{5 H}$, yellow arrow). Therefore, it is important to try different thresholding and smoothing methods in this plugin to determine the best parameters for analysis. If the automatic counting does not produce accurate numbers, the cell counter plugin can facilitate manual counting of cells or nuclei.

In cases utilizing transient transfection, images will often contain a mixture of cells expressing or not expressing a protein of interest (Figure 6). In this scenario, it is not always apparent which cells were responsible for creating areas of matrix degradation. This is especially true if the cells are migrating across the gelatin. To be consistent in the analysis, it is important to only measure the degraded areas directly underneath each cell. By thresholding to select the dark areas in the matrix and using the actin to generate cell outlines, only the degraded areas under the cells will be quantitated. This procedure will exclude degraded areas outside of cell boundaries from analysis (Figure 6F, arrow). The assay may require optimization to select a time point that allows sufficient time for degradation before the cells have had a chance to move.

Numerous methods have been developed to quantitate invadopodia formation and function. In addition to matrix degradation, other frequently reported parameters include determining the number of invadopodia per cell, the percentage of cells displaying invadopodia within a given population, and the number of "immature" or "pre" non-degrading invadopodia compared to "mature" invadopodia capable of degrading the $\mathrm{ECM}^{11,13,25,26}$. The method(s) of choice for invadopodia evaluation depend on inherent characteristics of each cell type. For instance, counting the number of invadopodia per cell or determining the percentage of cells containing invadopodia is a straightforward approach that works well if the analyzed cells contain just a few prominent invadopodia, but becomes more difficult in cells that have dozens of invadopodia or where invadopodia may be small and difficult to detect. Using the degradation assay makes it possible to calculate the percentage of pre-invadopodia vs. mature invadopodia in single cells or in a population by comparing the total number of cells with invadopodia to the percentage that are degrading matrix. If there is a discrepancy where fewer cells are degrading matrix compared to cells displaying invadopodia, it may indicate that these cells are forming pre-invadopodia that were incapable of matrix degradation at the time the cells were fixed.

Whatever method combination is chosen for analysis, it is important to quantify the desired invadopodia characteristics as objectively as possible. When collecting images on the microscope, choose fields by looking at cells (actin), rather than the fluorescent matrix, to avoid bias from preferentially selecting areas with high levels of degradation. Multiple images should be acquired to ensure a fair representation of the cell population. Images should also be acquired at an appropriate magnification. For uniform populations of cells, lower magnification can be used to collect more cells as long as the areas of degradation can still be resolved. Higher magnification images are preferred to measure areas under individual cells and to resolve individual invadopodia. When areas are being quantitated, thresholding images based on intensity is more objective than manually choosing the area of the matrix to measure. In all cases, a sufficient number of cells from multiple independent experiments should be analyzed to give statistically meaningful, reproducible results.

\section{Disclosures}

No conflicts of interest declared.

\section{Acknowledgements}

This work was supported by endowment funds from the West Virginia University Mary Babb Randolph Cancer Center. We thank Susette Mueller (Georgetown University) and Laura Kelley for early advice and assistance. The use of the West Virginia University Microscopy Imaging Facility (supported by the Mary Babb Randolph Cancer Center and, NIH grants P20 RR16440, P30 RR032138 and P30 GM103488) is gratefully acknowledged.

1. Ridley, A.J. Life at the leading edge. Cell. 145, 1012-1022, [pii] S0092-8674(11)00652-0 doi:10.1016/j.cell.2011.06.010 (2011).

2. Murphy, D.A. \& Courtneidge, S.A. The 'ins' and 'outs' of podosomes and invadopodia: characteristics, formation and function. Nat. Rev. Mol. Cell Biol. 12, 413-426, [pii] nrm3141 doi:10.1038/nrm3141 (2011).

3. Linder, S., Wiesner, C., \& Himmel, M. Degrading Devices: Invadosomes in Proteolytic Cell Invasion. Annu. Rev. Cell. Dev. Biol., doi:10.1146/ annurev-cellbio-092910-154216 (2010).

4. Chen, W.T., Chen, J.M., Parsons, S.J., \& Parsons, J.T. Local degradation of fibronectin at sites of expression of the transforming gene product pp60src. Nature. 316, 156-158 (1985).

5. Artym, V.V., Zhang, Y., Seillier-Moiseiwitsch, F., Yamada, K.M., \& Mueller, S.C. Dynamic interactions of cortactin and membrane type 1 matrix metalloproteinase at invadopodia: defining the stages of invadopodia formation and function. Cancer Res. 66, 3034-3043, [pii] 66/6/3034 doi:10.1158/0008-5472.CAN-05-2177 (2006).

6. Ayala, I., et al. Multiple regulatory inputs converge on cortactin to control invadopodia biogenesis and extracellular matrix degradation. J. Cell Sci., (2008).

7. Ammer, A.G., et al. Saracatinib impairs head and neck squamous cell carcinoma invasion by disrupting invadopodia function. J. Cancer Sci. Ther. 1, 52-61, doi:10.4172/1948-5956.1000009 (2009). 
8. Seals, D.F., et al. The adaptor protein Tks5/Fish is required for podosome formation and function, and for the protease-driven invasion of cancer cells. Cancer Cell. 7, 155-165 (2005).

9. Yamaguchi, H., et al. Molecular mechanisms of invadopodium formation: the role of the N-WASP-Arp2/3 complex pathway and cofilin. J. Cell Biol. 168, 441-452 (2005).

10. Kopp, P., et al. The kinesin KIF1C and microtubule plus ends regulate podosome dynamics in macrophages. Mol. Biol. Cell. 17, 2811-2823, [pii] E05-11-1010 doi:10.1091/mbc.E05-11-1010 (2006).

11. Oser, M., et al. Cortactin regulates cofilin and N-WASp activities to control the stages of invadopodium assembly and maturation. J. Cell. Biol. 186, 571-587 (2009).

12. Rasband, W.S. ImageJ. U.S. National Institutes of Health., (1997-2011)

13. Kelley, L.C., et al. Oncogenic Src requires a wild-type counterpart to regulate invadopodia maturation. J. Cell Sci. 123, 3923-3932, [pii] jcs.075200 doi:10.1242/jcs.075200 (2010).

14. Chen, W.T. \& Singer, S.J. Fibronectin is not present in the focal adhesions formed between normal cultured fibroblasts and their substrata. Proc. Natl. Acad. Sci. U.S.A. 77, 7318-7322 (1980).

15. Chen, W.T., Olden, K., Bernard, B.A., \& Chu, F.F. Expression of transformation-associated protease(s) that degrade fibronectin at cell contact sites. J. Cell Biol. 98, 1546-1555 (1984).

16. Bharti, S., et al. Src-dependent phosphorylation of ASAP1 regulates podosomes. Mol. Cell Biol. 27, 8271-8283 (2007).

17. Albrechtsen, R., Stautz, D., Sanjay, A., Kveiborg, M., \& Wewer, U. M. Extracellular engagement of ADAM12 induces clusters of invadopodia with localized ectodomain shedding activity. Exp. Cell Res. 317, 195-209, [pii] S0014-4827(10)00457-X doi:10.1016/j.yexcr.2010.10.003 (2011).

18. Scott, R.W., et al. LIM kinases are required for invasive path generation by tumor and tumor-associated stromal cells. J. Cell Biol. 191, 169-185, [pii] jcb.201002041 doi:10.1083/jcb.201002041 (2010).

19. Mueller, S.C., Yeh, Y., \& Chen, W.T. Tyrosine phosphorylation of membrane proteins mediates cellular invasion by transformed cells. J. Cell. Biol. 119, 1309-1325 (1992).

20. Bowden, E.T., Coopman, P.J., \& Mueller, S.C. Invadopodia: unique methods for measurement of extracellular matrix degradation in vitro. Methods Cell Biol. 63, 613-627 (2001).

21. Baldassarre, M., et al. Dynamin participates in focal extracellular matrix degradation by invasive cells. Mol. Biol. Cell. 14, 1074-1084 (2003).

22. Alexander, N.R., et al. Extracellular matrix rigidity promotes invadopodia activity. Curr. Biol. 18, 1295-1299, [pii] S0960-9822(08)01035-X doi:10.1016/j.cub.2008.07.090 (2008).

23. Artym, V.V., Yamada, K.M., \& Mueller, S.C. ECM degradation assays for analyzing local cell invasion. Methods Mol. Biol. 522, 211-219, doi:10.1007/978-1-59745-413-1 15 (2009).

24. Schoumacher, M., Goldman, R.D., Louvard, D., \& Vignjevic, D.M. Actin, microtubules, and vimentin intermediate filaments cooperate for elongation of invadopodia. J. Cell Biol. 189, 541-556, [pii] jcb.200909113 doi:10.1083/jcb.200909113 (2010).

25. Clark, E.S., Whigham, A.S., Yarbrough, W.G., \& Weaver, A.M. Cortactin is an essential regulator of matrix metalloproteinase secretion and extracellular matrix degradation in invadopodia. Cancer Res. 67, 4227-4235, [pii] 67/9/4227 doi:10.1158/0008-5472.CAN-06-3928 (2007).

26. Yamaguchi, H., et al. Phosphoinositide 3-kinase signaling pathway mediated by p110alpha regulates invadopodia formation. J. Cell Biol. 193, 1275-1288, [pii] jcb.201009126 doi:10.1083/jcb.201009126 (2011).

27. Li, A., et al. The actin-bundling protein fascin stabilizes actin in invadopodia and potentiates protrusive invasion. Curr. Biol. 20, 339-345, [pii] S0960-9822(09)02159-9 doi:10.1016/j.cub.2009.12.035 (2010).

28. Yamaguchi, H., et al. Phosphatidylinositol 4,5-bisphosphate and PIP5-kinase lalpha are required for invadopodia formation in human breast cancer cells. Cancer Sci. [pii] CAS1574 doi:10.1111/j.1349-7006.2010.01574.x (2010). 
Review

\title{
Tumor and Stromal-Based Contributions to Head and Neck Squamous Cell Carcinoma Invasion
}

\author{
Steven M. Markwell and Scott A. Weed * \\ Department of Neurobiology and Anatomy, Program in Cancer Cell Biology, Mary Babb Randolph \\ Cancer Center, West Virginia University, Morgantown, WV 26506, USA; \\ E-Mail: smarkwell@hsc.wvu.edu \\ * Author to whom correspondence should be addressed; E-Mail: scweed@hsc.wvu.edu; \\ Tel.: +1-304-293-3016; Fax: +1-304-293-4667.
}

Academic Editor: Hiromi I. Wettersten

Received: 12 January 2015 / Accepted: 15 February 2015 / Published: 27 February 2015

\begin{abstract}
Head and neck squamous cell carcinoma (HNSCC) is typically diagnosed at advanced stages with evident loco-regional and/or distal metastases. The prevalence of metastatic lesions directly correlates with poor patient outcome, resulting in high patient mortality rates following metastatic development. The progression to metastatic disease requires changes not only in the carcinoma cells, but also in the surrounding stromal cells and tumor microenvironment. Within the microenvironment, acellular contributions from the surrounding extracellular matrix, along with contributions from various infiltrating immune cells, tumor associated fibroblasts, and endothelial cells facilitate the spread of tumor cells from the primary site to the rest of the body. Thus far, most attempts to limit metastatic spread through therapeutic intervention have failed to show patient benefit in clinic trails. The goal of this review is highlight the complexity of invasion-promoting interactions in the HNSCC tumor microenvironment, focusing on contributions from tumor and stromal cells in order to assist future therapeutic development and patient treatment.
\end{abstract}

Keywords: invasion; HNSCC; tumor microenvironment; metastasis 


\section{Introduction}

Head and neck squamous cell carcinoma (HNSCC) is one of the most aggressive and invasive cancer types [1]. A common HNSCC hallmark is loco-regional invasion and metastasis to cervical lymph nodes, accounting for an $88 \%$ patient mortality rate in the two years following metastatic disease development [1]. Despite the long-held notion of genomic instability in advanced disease stages, recent studies have found no difference in the accumulation of mutations in tumors from patients with and without lymph node involvement [2]. This indicates that alterations other than mutations in signaling pathways likely account for progression from primary tumor to invasive and metastatic disease. The contributions towards metastatic disease arise both from changes in the behavior of tumor cells and interactions with various stromal components in the tumor microenvironment. The purpose of this review is to highlight the molecular and cellular mechanisms utilized by tumor cells and the associated microenvironment in promoting HNSCC invasiveness.

\section{Tumor Cell Contributions}

In HNSCC patients, two out of three individuals exhibit locoregional or distal metastasis at diagnosis, correlating with poor patient survival $[3,4]$. As in other carcinomas, HNSCC invasion involves a multi-step process that entails initial breaching of the basement membrane, tumor cell migration through the stromal extracellular matrix (ECM), intravasation into regional vasculature, and extravasation at the metastatic site. These stages frequently utilize proteolytic-mediated degradation of ECM proteins to facilitate tumor cell spreading [5-9].

\subsection{Cell-ECM Interactions}

The activity of several actin cytoskeletal-modulating proteins has been demonstrated to alter the invasive nature of HNSCC. The basement membrane and ECM are barriers that tumor cells must bypass in order to move into the surrounding stroma $[6,10,11]$. Tumor cell mediated proteolytic degradation of ECM components, globally or at focalized points termed invadopodia, is essential to the invasive process [6,10-12]. Invadopodia are actin-based membrane protrusions that mediate tumor cell dissemination by degrading restrictive ECM proteins through the action of matrix metalloproteinases (MMPs) [5,10-12]. Many MMPs are overexpressed in HNSCC, including the invadopodia-associated MMPs MMP-2, MMP-9, and MMP-14 [9,12,13]. Invadopodia comprise a central filamentous (F)-actin core surrounded by an integrin-based adhesion complex ring [6,10,11]. Cortactin and Arp2/3 complex are essential protein components involved in formation of the F-actin invadopodia core [14-17]. Cortactin is overexpressed in several cancer types including HNSCC, resulting in enhanced tumor cell motility and invasion [18-25]. Cortactin stabilizes actin branch points, binding to both the F-actin "mother" filament and Arp2/3 complex on the "daughter" filament [22,25-27]. The end result of this activity is enhanced invadopodia formation and maturation, leading to robust localized ECM degradation [22,25-27]. Further evidence indicates that cortactin overexpression correlates with lymph node involvement and metastases [28-30]. In addition to modulating cytoskeletal dynamics, cortactin facilitates localization and activation of MMP-14 (also termed membrane type 1-matrix metalloproteinase (MT1-MMP)) to invadopodia along with the secretion of MMP-2 and MMP-9 at sites of focalized degradation of ECM 
proteins $[13,31,32]$. The activity of MMP-14, MMP-2, and MMP-9 is significantly elevated in HNSCC cell lines with high metastatic potential and well as oral cancer patient samples with lymph node involvement $[12,33,34]$.

Several studies have demonstrated increased localization of the actin bundling protein fascin at the tumor invasive front [35,36]. Facin functions by bundling F-actin, which facilitates the formation of cellular protrusions necessary for cell-ECM interactions and cell motility [35-37]. Bundling of F-actin into parallel strands stabilizes filopodia and invadopodia, resulting in enhanced cell motility and localized ECM degradation [35-37]. Re-expression of fascin in facin-null SW1222 human colonic epithelial cells results in relocalization of integrin $\beta 1$ and vinculin to the leading edge of motile cells [38]. Overexpression of fascin in various tumors, including HNSCC, correlates with aggressive disease, high metastatic potential, and poor prognosis $[35,36]$.

Similarly, the serine/threonine kinase $\mathrm{p} 21$ protein $(\mathrm{Cdc} 42 / \mathrm{Rac})$-activated kinase (PAK1) is enriched at the invasive boarder of HNSCC tumors, and is essential for HNSCC invasion in vitro [39,40]. PAK1 resides in the cytoplasm, but can be detected at the leading edge of motile cells, focal adhesions, cell-cell junctions, and cortical actin structures [41-44]. PAKs phosphorylate several cytoskeletal protein targets, including vimentin, desmin, LIM kinase (LIMK), myosin light chain (MLC), and myosin light chain kinase (MLCK), where phosphorylation directly correlates with enhanced cellular motility [39,40]. PAK1-mediated MLCK phosphorylation reduces stress fiber formation, while PAK-1-mediated MLC phosphorylation induces contractility $[41,45,46]$. LIMK activation facilitates LIMK binding to the F-actin severing protein $\mathrm{ADF} /$ cofilin, inhibiting $\mathrm{ADF} /$ cofilin activity via phosphorylation to stabilize the $\mathrm{F}$-actin network $[41,47,48]$. The p41-ARC subunit of Arp2/3 complex can be directly phosphorylated by PAK1, activating Arp2/3 actin nucleation activity to enhance F-actin formation and increase cell motility [49,50]. This effect on actin network formation can also be accomplished through PAK1 phosphorylation of cortactin [49,51]. In addition to altering cytoskeletal dynamics, PAK1 has been implicated in the downregulation of cell-cell contacts. PAK1-mediated phosphorylation of the transcription factor Snail results in reduced expression of the epithelial cell-cell adhesion molecule epithelial (E)-cadherin [41,52]. Secretion of MMP-1, MMP-3, and MMP-9 correlates directly with PAK1 expression, suggesting that the activity of PAK1 may enhance proteolytic degradation of ECM [53,54]. Overexpression of PAK1 in various tumors, including HNSCC, correlates with aggressive disease and poor prognosis $[39,40]$.

The calcium binding proteins S100A8 and S100A9 belong to a family of low-molecular-weight cytoplasmic proteins primarily detected as a S100A8/A9 heterodimer termed calprotectin [55-58]. Expression and secretion of S100A8/A9 is associated with chronic inflammation and is released from tumor cells in response to hypoxic stress [55]. While S100A8 and S100A9 are overexpressed in a multitude of cancers, their expression is suppressed in HNSCC $[55,59,60]$. Certain studies have demonstrated a pro-apoptotic role of S100A8/A9, inducing pro-caspase-3 cleavage and downregulating expression of anti-apoptotic members of the Bcl family, Bcl2 and Bcl- $\mathrm{X}_{\mathrm{L}}[55,61]$. The ability of S100A8/A9 to induce an apoptotic response, rather than the role in inflammatory signaling, is the most likely reason that expression of these proteins is lost in HNSCC. In addition to inflammatory signaling and apoptotic response, S100A8/A9 regulates the expression and secretion of MMP-2, representing a potential upstream therapeutic target $[59,60]$. Thus, calprotectin may serve a dual role in HNSCC by preventing apoptosis while facilitating MMP-2-driven metastatic dissemination. 
In order to monitor the surrounding ECM, cells form actin-rich protrusions that in a migratory cell contact the ECM to form structures known as focal adhesions. Focal adhesions contain the well-characterized cytoskeletal proteins talin, paxillin, $\alpha$-actinin, vinculin and focal adhesion kinase (FAK) [62-64]. Focal adhesions serve as intermediary structures by linking the actin cytoskeleton within the cell to the ECM surrounding the cell by interacting with the cytoplasmic domains of the integrin class of transmembrane ECM receptors [62,65-68]. Integrin extracellular domains directly bind ECM proteins, including fibronectin, laminin, collagen I and collagen IV. [62,65-68]. FAK activation precedes focal contact formation and facilitates focal adhesion maturation through phosphorylation of Rho guanine nucleotide exchange factors and phosphatidylinositol phosphate kinase isoform $\gamma$, which enhances talin binding to integrin cytoplasmic domains [66,69]. Regulation of focal adhesion disassembly at the trailing edge by FAK dramatically alters cellular motility [66,70,71]. FAK overexpression occurs early in HNSCC development, correlating with increased tumor cell invasion and lymph node metastasis, partially through an increase in MMP-2 and MMP-9 secretion [67-69]. As such, FAK has become a therapeutic target in many tumor types, where pharmacological inhibition of FAK tyrosine kinase activity results in decreased tumor cell invasion [72-75].

Phospholipase D (PLD1), mediates the hydrolysis of phosphatidyl choline into choline and the second messenger phosphatidic acid [49,76,77]. Phosphatidic acid is further hydrolyzed by phosphatidic acid phosphohydrolases to generate diacylglycerol and lysophosphatidic acid (LPA), the latter being a key mediator of inflammatory response and has been implicated in oncogenesis and metastatic progression $[10,76]$. In addition, LPA activates the Rho family of cytoskeletal regulatory GTPases, facilitating the formation of filopodia, lamellipodia, and stress fibers essential for cell movement [49,76]. PLD1 has been shown to drive stress fiber and focal adhesion formation in HeLa cells [78]. PLD1 is overexpressed in several cancers including HNSCC, where it activates Src kinase and mitogen activated protein kinase (MAPK), driving invadopodia formation, maturation, and tumor cell invasion [79-82]. Due to the numerous migratory and invasive signaling networks stimulated by PLD1 and PLD1 substrates, PLD1 represents a viable upstream target for limiting tumor spread and metastatic progression. To this end, the PLD1 inhibitors quercetin, ML298, and ML299 decrease U87 glioblastoma cell invasion in in vitro assays [83,84]. These data support further investigation into PLD1 inhibitor efficacy in suppressing HNSCC invasion.

The phosphoinsositide-3-kinase (PI3K) family of kinases are among the most frequently altered oncongenic drivers in cancer $[85,86]$. Genomic alteration of PI3K occurs in approximately $31 \%$ of HNSCC tumors $[85,86]$. The PI3K class IA isoforms, $\mathrm{p} 110 \alpha, \mathrm{p} 110 \beta$, and $\mathrm{p} 110 \delta$ lie directly downstream of many oncogenic receptor tyrosine kinases, including epidermal growth factor receptor (EGFR), human epidermal growth factor receptor 3 (HER3), Met, platelet-derived growth factor receptor (PDGFR), vascular endothelial growth factor receptor (VEGFR), and insulin-like growth factor receptor 1 (IGF-1R) [85,87]. The PI3K isoform $\mathrm{p} 110 \alpha$ is the most commonly overexpressed family member in HNSCC, acting upstream of Cdc42, Rac, and Rho kinases, to enhance filopodia and lamellipodia formation resulting in increased cellular motility [85,86,88-90].

Despite the expression of several fibroblast growth factor (FGF) receptors in HNSCC, surprisingly little investigation has focused on secretion of the FGF gene products FGF-3, FGF-4, and FGF-19 located within the 11q13 amplified region found in nearly a third of HNSCC patient samples [91,92]. Studies have focused on FGF-2 and FGF-binding protein, identifying autocrine loops with these FGF receptors that correlate with enhanced HNSCC invasion [92,93]. Given the establishment of these 
autocrine loops and the potential for these secreted FGFs to attract fibroblasts into proximity with HNSCC cells (see below), further investigation into the 11q13 amplified FGFs is warranted to determine if these proteins contribute to HNSCC metastatic progression.

\subsection{Cell-Cell Interactions}

In addition to enhanced motility at the individual cellular level, the mode of tumor cell migration also impacts local invasion and metastasis. Tumor cells can invade as individual cells, displaying either mesenchymal or amoeboid migration depending on intercellular signaling events, which result in poorly differentiated tumors due to the intermingling of individual invasive tumor cells with the stromal tissue [94,95]. Other tumor cells utilize multicellular or collective invasion, maintaining tumor cell-cell junctions, resulting in moderately to well differentiated tumors as the invasive tumor cells can be distinguished from the surrounding tissue [94,95]. In histological HNSCC samples displaying a broad invasive front, tumors remain well-to moderately-differentiated due the tumor cells being easily distinguished from the surrounding tissue by retaining membranous E-cadherin staining. These characteristics indicate that such tumors undergo collective invasion. In addition, cases where tumors display individual finger-like invasive fronts, tumors are poorly differentiated as individual tumor cells are intermingled with stromal cells. These invasive tumor cells show reduced E-cadherin staining, with notable increases in both phospho-Src and vimentin that represent a more mesenchymal invasion modality [96]. Patients with elevated phospho-Src and vimentin have direct correlation with greater lymph node involvement and advanced tumor stage [96]. Although E-cadherin is not essential to collective invasion, maintenance of cell-cell adhesions and an epithelial phenotype allow for multicellular invasive clusters to migrate simultaneously [94,95].

In addition to direct cell-cell contact, tumor cells interact through autocrine and paracrine signaling networks. EGFR is overexpressed in greater than $95 \%$ of HNSCC patient samples, and phosphorylation of the downstream effector Src kinase correlates with poorly differentiated HNSCC, lymph node involvement, and poor patient outcome [97-99]. Recent studies indicate that there are two distinct subpopulations within most HNSCC tumors, in which E-cadherin and vimentin are inversely expressed [100-102]. These two subpopulations demonstrate plasticity in regenerating heterogeneity in culture and xenograft tumors derived from single subpopulations, but respond differentially to various chemotherapeutic agents [100-102]. Expression of EGFR is variable in these subpopulations, correlating inversely with vimentin expression, suggesting a potential mechanism for acquired EGFR inhibitor resistance that is observed in the clinic [100-102]. Another receptor tyrosine kinase, tyrosine receptor kinase B (TrkB), is expressed in more than half of HNSCC patient tumors. TrkB activates the transcription factors Snail and Twist, driving the epithelial to mesenchymal transition (EMT) and enhancing tumor cell invasion [103]. These data collectively support the idea that deterioration of cell-cell contacts drives a drug resistant and more invasive phenotype in HNSCC.

\subsection{Angiogenesis and Neo-Vascularization}

Angiogenesis not only supplies growing tumors with requisite nutrition, but also provides cells at the tumor periphery a route to disseminate into surrounding tissues and the rest of the body. In addition to MMPs, HNSCC cells secrete a variety of pro-angiogenic factors that recruit endothelial cells into 
the local tumor microenvironment, resulting in formation of a leaky capillary bed that facilitates tumor cell intra- and extravasation. Two key angiogenic paracrine signaling profiles have been proposed for HNSCC cells. The first utilizes excess secreted VEGF, FGF-2, with small amounts of interleukin (IL)-8. The second mainly consists of IL-8, with lesser amounts of VEGF and FGF-2 [104-106]. In addition, primary HNSCC tumor cell cultures, tissue specimens, and established cells lines have enhanced secretion of VEGF and/or PDGF-AB, with lesser, yet still elevated, secretion of granulocyte colony stimulating factor (G-CSF) and granulocyte macrophage (GM)-CSF [105,107]. Increased secretion of these cytokines drives HNSCC tumor angiogenesis and corresponds with decreased patient survival [105]. Furthermore, oral SCC tissue samples display enhanced lymphatic microvessel density in the presence of VEGF, PDGF, basic FGF, hepatocyte growth factor (HGF) and IGF-1 [108,109]. Enhanced primary tumor lymphatic and blood microvessel density in response to these secreted factors correlates with lymph node metastasis and invasive tumor margins [110,111]. Endothelial cell recruitment and formation of an immature vascular network around the tumor in response to HNSCC cell angiogenic secretions are therefore prime contributors for providing essential routes for primary tumor cell invasion and metastatic dissemination.

\subsection{Metastasis to Distant Sites}

Once tumor cells reach the blood or lymphatic vasculature, they must survive in circulation until they reach lymph nodes or other metastatic sites. While little has been elucidated about such circulating tumor cells (CTCs) in HNSCC, the amount of HNSCC CTCs rises significantly in stage IV tumors, correlating directly with increased metastasis and inversely with therapeutic response [112,113]. HNSCC CTCs are not well defined, and are typically characterized as cells expressing epidermal cell adhesion molecule (EpCAM) or cytokeratin (CK) 8, CK18, or CK19 in blood samples [112,113]. One study found that IL-6 enhanced survival and self-renewal of the aldehyde dehydrogenase $(\mathrm{ALDH}){ }^{\text {high }} \mathrm{CD} 44^{\text {high }}$ cell population, representing a potential cancer stem cell (CSC) subpopulation sufficient to reconstitute a tumor when transplanted into a mouse xenograft model [114]. This same CSC subpopulation is resistant to cisplatininduced cell death [115]. There is evidence that indicates EGFR, TrkB, and IL-1 $\beta$ are essential to maintaining a mesenchymal subpopulation associated with chemotherapeutic resistance in HNSCC [103,116,117]. Other studies suggest that these mesenchymal-like cells can recapitulate the epithelial population of a tumor following chemotherapeutic therapy, potentially representing the HNSCC tumor equivalent to the $\mathrm{CD} 4^{+} / \mathrm{CD} 24^{-}$stem-like subpopulation in breast carcinomas [100-102]. It remains unclear if these mesenchymal-like cells, CSCs and CTCs are the same or unique HNSCC subpopulations, but all show tumor initiating capacity that can be utilized to form metastases [101-103,114,115,117]. Once these tumor initiating cells (TICs) reach the metastatic site, they must first extravasate, a process aided by the local endothelial cells [114]. Following extravasation, some TICs differentiate back into the more epithelial phenotype that makes up the majority of the tumor mass, while other TICs undergo self-renewal to maintain the subpopulation [100-102,114]. Reconstituting the entire tumor mass allows the tumor to grow rapidly, taking advantage of the hospitable metastatic niche since the epithelial cell phenotype shows enhanced proliferation rates as compared to TICs [101,117]. While CTCs, CSCs, and mesenchymal-like cells represent resistant subpopulations potentially capable of initiating recurrence 
and correlates with invasive and metastatic disease, investigation into this aspect of HNSCC progression for therapeutic targeting has become an important newly emerging field [100,103,112,113,116].

\section{Stromal Cell Contributions}

In addition to carcinoma cells, various cellular and acellular stromal components contribute to promoting and maintaining HNSCC invasion. Deposition of specific ECM proteins (collagen IV, collagen XVII, fibronectin, and laminin) is enhanced in HNSCC tumors and serve as a chemo-attractant for HNSCC cells in various in vitro invasion assays [118-120]. As HNSCC tumors progress towards metastatic disease, non-tumor cell types from the associated stroma have been shown to have direct and indirect roles in facilitating HNSCC invasion.

\subsection{Mast Cells}

Mast cells are part of the immune myeloid lineage that mediate innate and acquired immune responses through granule exocyctosis, releasing histamine, serine proteases, carboxypeptidase A (CPA1), proteoglycans, prostaglandin $\mathrm{D}_{2}\left(\mathrm{PGD}_{2}\right)$, leukotriene $\mathrm{C}_{4}\left(\mathrm{LTC}_{4}\right)$, tumor necrosis factor (TNF)- $\alpha, \mathrm{GM}-\mathrm{CSF}$, IL-3, IL-4, IL-5, IL-6, IL-8, and IL-16 [121]. During advanced HNSCC stages, where the tumor has spread to loco-regional or distal lymph nodes, mast cells accumulate in the tumor stroma, with their presence directly correlating with increased angiogenesis [104,122,123]. How HNSCC tumors suppress rapid mast cell activation in response to immunoglobulin $\mathrm{E}$ or $\mathrm{CD} 32$ binding to $\mathrm{F}_{\mathrm{c} \varepsilon} \mathrm{RI}$ or $\mathrm{F}_{\mathrm{C} \gamma} \mathrm{RIIb}$ respectively remains to be elucidated, but may occur by blocking $F_{c \varepsilon} R I$ activation on mast cells [121]. Additionally, heparanase, an enzyme involved in cleavage and remodeling heparin sulfate proteoglycans in the ECM, accumulates at the HNSCC invasive front, and is a marker of poor prognosis for lymph node metastasis and tumor recurrence [124]. Mast cells, along with tumor infiltrating neutrophils, endothelial cells, and macrophages exhibit heparanase activity [124,125]. However, since mast cells also secrete large amounts of heparin, they are the cell type that is likely responsible for invasion-associated heparanase activity in the tumor microenvironment. While the main contribution of mast cells to tumor progression may be inflammation-mediated recruitment of other cell types into the microenvironment, their presence also facilitates HNSCC tumor neo-vascularization and dissemination to loco-regional lymph nodes.

\subsection{Neutrophils}

Neutrophils are another component of the immune system that contributes to the innate immune response [126]. Neutrophils are recruited to the tumor microenvironment by pro-inflammatory signals, including IL-8, transforming growth factor (TGF)- $\beta$, IL-4, IL-10, IL-13, GM-CSF, and TNF- $\alpha[127,128]$. Following recruitment to the tumor microenvironment, neutrophils secrete VEGF-A, stimulating neovascularization through endothelial cell recruitment and proliferation, which can be abrogated via anti-VEGF-A antibodies or angiostatin treatment [128-130]. Additionally, neutrophil-derived HGF and MMP-9 facilitate tumor cell migration and invasion towards the newly formed vascular bed [128]. In this context, neutrophils bridge the gap between the growing tumor mass and the local vasculature, 
bringing in endothelial cells to an area of growing hypoxia while promoting a chemotactic invasive phenotype in the tumor cells.

\subsection{Macrophages}

Macrophages belong to the myeloid lineage of the immune system [131]. Macrophages play a direct role in immune surveillance through endocytosis of pathogens and cellular debris [131]. Tumor associated macrophage (TAM) infiltration into the tumor microenvironment correlates with lymph node involvement, tumor stage, and extracapsular spread [132-134]. Once TAMs arrive in the tumor microenvironment, TAM secretions set up several paracrine signaling loops that drive tumor cell invasion and metastasis. In one loop, TAMs secrete EGF stimulating tumor cell growth, migration, and invasion. Correspondingly, HNSCC cells secrete CSF-1 that drives further TAM proliferation and tumor infiltration [135-137]. In another loop, TAMs secrete macrophage migration inhibitory factor (MMIF), attracting and activating neutrophils, which subsequently interact with HNSCC cells as described above [128-130,138]. In response to HNSCC secreted paracrine factors, TAMs develop podosomes, capable of assisting tumor cells breach the basement membrane and enter the vascular or lymphatic network [133,139]. Similar to invadopodia, podosomes are membrane protrusions containing an actin-rich core surrounded by an integrin ring that mediates interaction with the ECM [140]. Podosomes are formed at the leading edge of motile cells and contribute to cellular motility, simultaneously allowing cells to adhere to the ECM and initiating acto-mysoin contractility to pull the cell body forward $[139,140]$. Podosomes can also localize MMPs, including MMP-2, MMP-9, and MT1-MMP to proteolytically degrade and rearrange the ECM [140,141]. TAMs also secrete the chemotactic factor macrophage inflammatory protein (MIP)-3 which drives HNSCC cell migration and invasion [142]. Through these signaling pathways, macrophages are able to promote and maintain the HNSCC invasive phenotype, assist in basement membrane breakdown and recruitment of other cell types into the tumor microenvironment.

\subsection{Endothelial Cells}

While endothelial cells play a major role in vascularization of the growing tumor mass, emerging evidence demonstrates a novel role for endothelial cells in facilitating tumor cell invasion. The chemotactic factors VEGF, TNF- $\alpha$, and TGF- $\beta$ induce podosome formation in endothelial cells along the invasive tumor front $[141,143-146]$. This allows endothelial cells to reach hypoxic tumor regions, facilitating breakdown of basement membrane encapsulating the primary tumor. Once endothelial cells come into direct contact with tumor cells, endothelial cell Notch activation in response to HNSCC-derived Notch ligand Jagged 1 drives capillary-like sprout formation and neovascularization of the expanding tumor mass [147]. The combined effort of endothelial cell-mediated rearrangement of the microenvironment to promote tumor cell access to the vascular network makes endothelial cells important contributors to HNSCC tumor progression.

\subsection{Fibroblasts}

The desmoplastic response is a hallmark of cancer progression, where secretion and restructuring of ECM proteins drives tumor cell proteolytic invasion and production of "tracks" for proteolytic-independent 
invasion modes [148,149]. Fibroblasts are specialized for this task, as they can degrade and rearrange a variety of ECM proteins including type I and IV collagens, laminin, and fibronectin $[145,149,150]$. Integrin $\alpha 6$ expression allows such cancer associated fibroblasts (CAFs) to bind the basement membrane protein laminin, enabling CAF-mediated proteolytic laminin degradation [140,151]. Expression of integrin $\alpha 6$ in CAFs has been linked to poor prognosis in oral cancer patients [151]. Fibroblast-mediated proteolytic cleavage of ECM proteins requires direct contact with tumor cells or binding of HNSCC secreted endothelin-1 (ET-1), leading to localization of a disintegrin and metalloprotease (ADAM)-12 and ADAM-17 at fibroblast podosomes, followed by secretion and activation of MMP-2 and MMP-9 from carcinoma cells and CAFs [34,152-154]. Other studies suggest that chemokine C-X-C motif receptor type 4 (CXCR4) binding to CAF-secreted chemokine C-X-C motif ligand 12 (CXCL12) initiates carcinoma derived MMP-9 secretion in the tumor microenvironment [34,155]. Regardless of the source of MMP secretion, total MMP levels and the ratio of activated MMPs to total MMP concentration compared with adjacent normal tissue positively correlates with lymph node involvement $[155,156]$. As a result, the HNSCC stroma is enriched in infiltrating CAFs, with the highest concentrations accumulating near the invasive front of the tumor [24,29,157]. Infiltrating CAFs have several characteristics of myofibroblasts, including enhanced proliferation and motility, expression of cytokeratins, vimentin, and $\alpha$-smooth muscle actin (SMA), and secretion of MMP-2 and HGF $[122,158,159]$. CAF MMP secretion facilitates ECM degradation and remodeling, whereas HGF enhances HNSCC cell motility $[122,158,159]$. In turn, enhanced CAF proliferation and motility allows the CAF population to expand and spread in accordance with the growing invasive tumor front $[122,158,159]$. The adaptation of HNSCC CAFs with myofibroblast characteristics results in extracapsular tumor cell spread, increased invasion, and lymph node metastasis [160]. Orthotopic floor of mouth co-injection of HNSCC cells with CAFs or normal fibroblasts in mice indicates that CAFs contribute significantly to lymph node and distal metastatic disease [161]. The net results of fibroblasts in the tumor microenvironment is rearrangement of ECM proteins, allowing fibroblasts to lead tumor cells into surrounding tissues or paving pathways in the stroma for invasive tumor cells to follow. Additionally, TGF- $\beta$ and miR-210 induced CAF senescence promotes fibroblast MMP-2 secretion and tumor cell EMT, enhancing in vitro tumor cell invasion [151,162-164]. Further evidence indicates that coinjection of tumor cells with senescent CAFs promotes xenograft engraftment and tumor growth [165-167]. These activities ultimately result in facilitating HNSCC metastatic progression.

\section{Anti-Metastatic Therapeutic Approaches}

While indolent primary HNSCC tumors are typically treated by surgical resection and/or radiation therapy, the treatment of invasive and metastatic disease is more complex. The development of preventative anti-metastatic therapies holds promise to broaden patient treatment options and improve survival rates. Many recent anti-metastatic treatments have been aimed at Src kinase due to the essential role Src plays in cancer cell motility and invadopodia formation, as well as the multitude of overexpressed upstream transmembrane receptors that activate Src in tumors [25-27]. Initial in vitro studies using saracatinib (AZD0530) resulted in decreased MMP-9 activation and ECM degradation in established HNSCC cell lines, and also reduced invasion in HNSCC cells lines from primary tumors and matched lymph node metastases in combination with the phospholipase C inhibitor U73122 [168,169]. Another 
combination study showed that saracatinib with the EGFR small molecule inhibitor gefitinib suppressed HNSCC cell invasion in vitro to a greater extent than either drug alone [170]. However, a subsequent Phase II trial of saracatinib resulted in no therapeutic benefit in either recurrent or metastatic HNSCC [171]. Treatment of HNSCC cell lines with the Src/Abl small molecule inhibitor dasatinib (BMS-354825) decreased migration and invasion while blocking the G1-S transition [172]. A Phase II clinical trial of dasatinib alone also failed to show clinical benefit to patients with late stage HNSCC [173]. These trial results clearly demonstrate that targeting Src is insufficient to prevent HNSCC progression, prompting the need to evaluate additional pro-invasive oncogenic targets. The activity of another oncogenic tyrosine kinase, Abl, downstream of EGFR and Src kinase facilitates invadopodia formation and promotes tumor cell invasion and metastasis [30,174-178]. In vitro treatment with the Abl family inhibitor imatinib mesylate (STI571; Gleevac) resulted in enhanced HNSCC cell invasion, opposite of what has been observed in invasive breast cancer [179]. Imatinib mesylate stimulates HNSCC shedding of heparin-binding EGF, which activates EGFR on the HNSCC cell surface, driving invadopodia formation and ECM degradation [179]. A phase II trial of imatinib mesylate and docetaxel for patients with metastatic non-small-cell lung carcinoma and HNSCC found no clinical benefit and closed early due to significant toxicity from this drug regimen [180]. Cetuximab (IMC-C225), an anti-EGFR humanized monoclonal antibody, shows multifaceted benefit in HNSCC by blocking proliferation, angiogenesis and metastasis while increasing tumor cell apoptosis [181-183]. Phase II clinical trials for patients with late stage HNSCC showed partial response to cetuximab alone in a small patient subset, while complete response was observed in the majority of patients when cetuximab was used in combination with cisplatin, fluorouracil, and radiotherapy [184,185]. The Erbitux in First-Line Treatment of Recurrent or Metastatic Head and Neck Cancer (EXTREME) Phase III trial showed significant increases in overall survival, progression-free survival, and response rate for the combination of cetuximab and platinum/5-fluorouralcil compared with platinum/5-fluorouracil alone [186-189]. While these trials did not directly investigate an anti-metastatic role for cetuximab, it is a promising advance in HNSCC treatment. Another study found that the potassium ionophore antibiotic salinomycin significantly inhibited growth of the cisplatin-resistant mesenchymal-like HNSCC subpopulation, likely through induction of apoptosis [101,190]. These data demonstrate a potential mechanism for targeting a drug resistant, highly mobile subpopulation that has been implicated in metastatic dissemination as well as disease recurrence [100-102]. While these initial studies have demonstrated some efficacy in patients with advanced disease, direct anti-invasive and antimetastatic therapeutic targeting continues to remain elusive in HNSCC.

\section{Conclusions}

HNSCC tumors contain a host of aberrant signaling pathways, from cytoskeletal modulation responsible for driving increased invasion to promoting tumor cell survival in the circulation. Interactions with the surrounding ECM as well as between individual tumor cells influences the ability of HNSCC cells to invade into the surrounding tissue and eventually to other parts of the body, predominantly the cervical lymph nodes. Changes in cell-cell adhesions along with alterations in cellular morphology allow HNSCC cells to undergo a variety of invasive patterns. Additionally, HNSCC cells utilize various autocrine and paracrine secreted factors in order to optimize tumor dissemination, whether through neovascularization by endothelial cells or rearrangement of ECM protein by local fibroblasts. 
The tumor microenvironment, depicted in Figure 1, is therefore a complex, dynamic system, complicating our understanding of tumor behavior and potential therapeutic interventions.

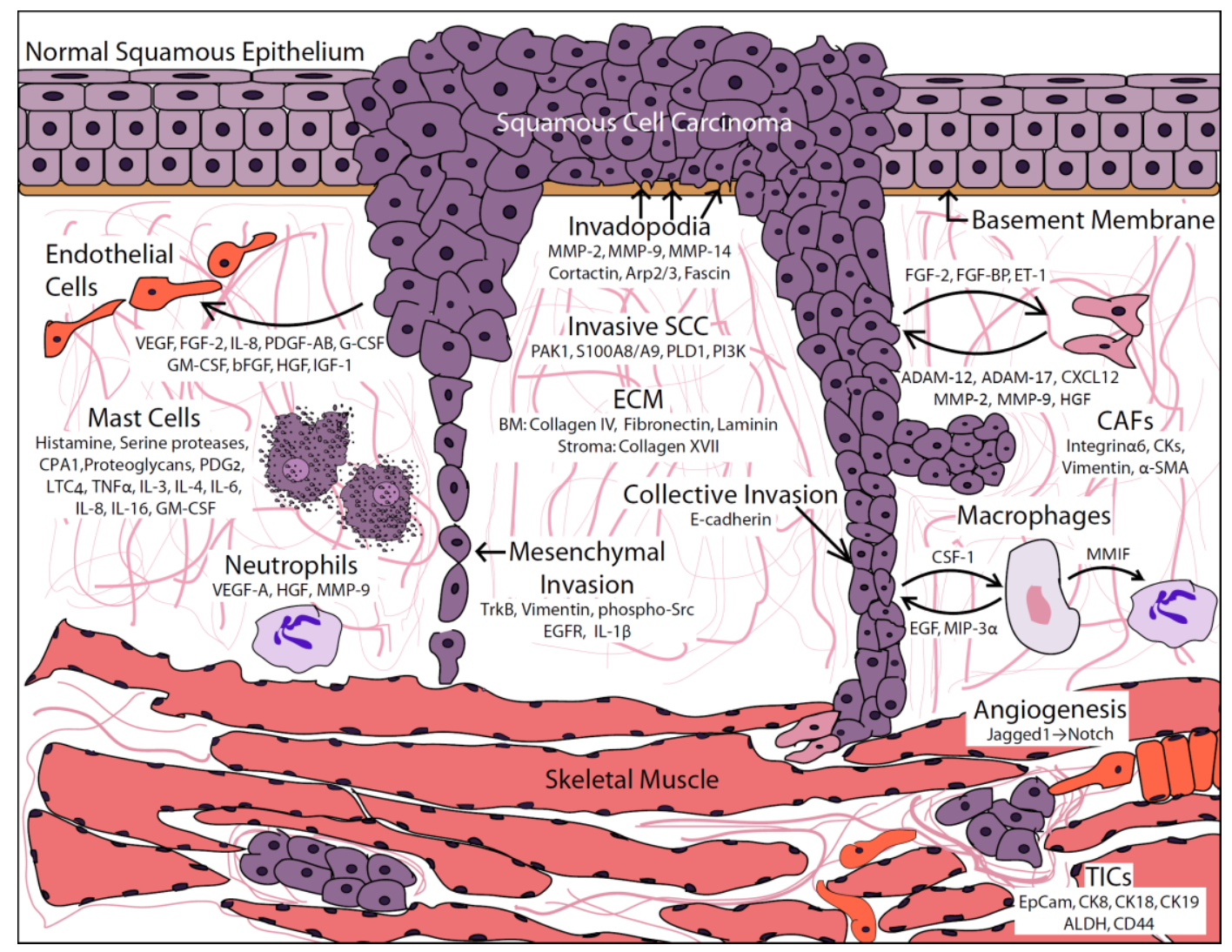

Figure 1. Diagram of tumor and stromal-based contributions that promote head and neck squamous cell carcinoma (HNSCC) invasion. Depicted is an invasive HNSCC tumor cell mass invading into the surrounding stroma and muscle tissue of the oral tongue. Specific cell types and their respective secreted protein contributions are detailed, demonstrating subsequent cellular responses and paracrine signaling networks. See text for additional detail.

Use of conventional wet-bench-based cell and xenograft assays cannot incorporate the breath of tumor-stromal interactions that occur in patient tumors. The inability of these models to accurately guide pharmaceutical intervention development, as witnessed by the lack of successful clinical trials to date, is a testament to the complexity and difficulty of targeting HNSCC invasion and metastasis. This is likely due to the multitude of pro-invasive signaling networks in HNSCC cells and various tumor-stromal interactions. Therefore, in order to make meaningful advances in the treatment of HNSCC invasion, new model systems need to be developed that include, or at least consider, all of the intracellular, cell-cell, and cell-matrix contributions from carcinoma cells and corresponding tumor-associated stromal cells found in patient tumors.

\section{Acknowledgments}

This work was supported by the Mary Babb Randolph Cancer Center, Department of Neurobiology and Anatomy and Office of Research and Graduate Education at West Virginia University. Additional support from the National Institute of General Medical Sciences, U54GM104942 is gratefully 
acknowledged. The content is solely the responsibility of the authors and does not necessarily represent the official views of the NIH.

\title{
Author Contributions
}

S.M.M. and S.A.W. wrote the manuscript.

\begin{abstract}
Abbreviations
ALDH, aldehyde dehydrogenase; ADAM-12, a disintegrin and metalloprotease 12; ADAM-17, a disintegrin and metalloprotease 17; bFGF, basic fibroblast growth factor; CAF, cancer associated fibroblast; CK, cytokeratin; CK8, cytokeratin 8; CK18, cytokeratin 18; CK19, cytokeratin 19; CSC, cancer stem cell; CTC, circulating tumor cell; CXCL12, chemokine C-X-C motif ligand 12; CXCR4, chemokine C-X-C motif receptor type 4; E-cadherin, epithelial-cadherin; ECM, extracellular matrix; EGF, epidermal growth factor; EGFR, epidermal growth factor receptor; EMT, epithelial to mesenchymal transition; EpCAM, epidermal cell adhesion molecule; ET-1, endothelin-1; F-actin, filamentous-actin; FAK, focal adhesion kinase; FGF-2, fibroblast growth factor 2; FGF-3, fibroblast growth factor 3; FGF-4, fibroblast growth factor 4; FGF-19, fibroblast growth factor 19; FGF-BP, fibroblast growth factor binding protein; G-CSF, granulocyte colony stimulating factor; GM-CSF, granulocyte macrophage colony stimulating factor; HER3, human epidermal growth factor receptor 3; HGF, hepatocyte growth factor; HNSCC, head and neck squamous cell carcinoma; IGF-1, insulin-like growth factor 1; IGF-1R, insulin-like growth factor receptor 1 ; IL-1 $\beta$, interleukin $1 \beta$; IL-3, interleukin 3; IL-4, interleukin 4; IL-5, interleukin 5; IL-6, interleukin 6; IL-8, interleukin 8; IL-10, interleukin 10; IL-13, interleukin 13; IL-16, interleukin 16; LIMK, LIM kinase; LTC4, leukotriene $\mathrm{C}_{4}$; MAPK, mitogen activated protein kinase; MIP-3 $\alpha$, macrophage inhibitor protein $3 \alpha$; MLC, myosin light chain; MLCK, myosin light chain kinase; MMIF, macrophage migration inhibitory factor; MMP, matrix metalloproteinase; MMP-1, matrix metalloproteinase 1; MMP-2, matrix metalloproteinase 2; MMP-3, matrix metalloproteinase 3; MMP-9, matrix metalloproteinase 9; MMP-14, matrix metalloproteinase 14; MT1-MMP, membrane type 1-matrix metalloproteinase; PAK1, p21 protein (Cdc42/Rac)-activated kinase 1; PDGF, platelet-derived growth factor; PDGF-AB, platelet-derived growth factor AB; PDGFR, platelet-derived growth factor receptor; $\mathrm{PGD}_{2}$, prostaglandin $\mathrm{D}_{2}$; $\mathrm{PI} 3 \mathrm{~K}$, phosphoinsositide-3-kinase; TAM, tumor associated macrophage; TGF- $\beta$, transforming growth factor $\beta$; TIC, tumor initiating cell; TNF- $\alpha$, tumor necrosis factor $\alpha$; TrkB, tyrosine receptor kinase B; VEGF, vascular endothelial growth factor; VEGF-A, vascular endothelial growth factor A; VEGFR, vascular endothelial growth factor receptor.
\end{abstract}

\section{Conflicts of Interest}

The authors declare no conflict of interest.

\section{References}

1. Probert, J.C.; Thompson, R.W.; Bagshaw, M.A. Patterns of spread of distant metastases in head and neck cancer. Cancer 1974, 33, 127-133. 
2. Onken, M.D.; Winkler, A.E.; Kanchi, K.-L.; Chalivendra, V.; Law, J.H.; Rickert, C.G.; Kallogjeri, D.; Judd, N.P.; Dunn, G.P.; Piccirillo, J.F.; et al. A surprising cross-species conservation in the genomic landscape of mouse and human oral cancer identifies a transcriptional signature predicting metastatic disease. Clin. Cancer Res. 2014, 20, 2873-2884.

3. Leemans, C.R.; Braakhuis, B.J.M.; Brakenhoff, R.H. The molecular biology of head and neck cancer. Nat. Rev. Cancer 2011, 11, 9-22.

4. Koontongkaew, S. The tumor microenvironment contribution to development, growth, invasion and metastasis of head and neck squamous cell carcinomas. J. Cancer 2013, 4, 66-83.

5. Bowden, E.T.; Barth, M.; Thomas, D.; Glazer, R.I.; Mueller, S.C. An invasion-related complex of cortactin, paxillin and PKCmu associates with invadopodia at sites of extracellular matrix degradation. Oncogene 1999, 18, 4440-4449.

6. Branch, K.M.; Hoshino, D.; Weaver, A.M. Adhesion rings surround invadopodia and promote maturation. Biol. Open 2012, 1, 711-722.

7. Yamaguchi, H.; Wyckoff, J.; Condeelis, J. Cell migration in tumors. Curr. Opin. Cell Biol. 2005, 17, 559-564.

8. Baldassarre, M.; Pompeo, A.; Beznoussenko, G.; Castaldi, C.; Cortellino, S.; McNiven, M.A.; Luini, A.; Buccione, R. Dynamin participates in focal extracellular matrix degradation by invasive cells. Mol. Biol. Cell 2003, 14, 1074-1084.

9. Leong, H.S.; Robertson, A.E.; Stoletov, K.; Leith, S.J.; Chin, C.A.; Chien, A.E.; Hague, M.N.; Ablack, A.; Carmine-Simmen, K.; McPherson, V.A.; et al. Invadopodia are required for cancer cell extravasation and are a therapeutic target for metastasis. Cell Rep. 2014, 8, 1-13.

10. Lohmer, L.L.; Kelley, L.C.; Hagedorn, E.J.; Sherwood, D.R. Invadopodia and basement membrane invasion in vivo. Cell Adh. Migr. 2014, 8, 246-255.

11. Paz, H.; Pathak, N.; Yang, J. Invading one step at a time: The role of invadopodia in tumor metastasis. Oncogene 2014, 33, 4193-4202.

12. Rosenthal, E.; Matrisian, L.M. Matrix metalloproteases in head and neck cancer. Head Neck 2006, 28, 639-648.

13. Clark, E.S.; Whigham, A.S.; Yarbrough, W.G.; Weaver, A.M. Cortactin is an essential regulator of matrix metalloproteinase secretion and extracellular matrix degradation in invadopodia. Cancer Res. 2007, 67, 4227-4235.

14. Wu, H.; Parsons, J.T. Cortactin, an 80/85-Kilodalton pp60Src substrate, is a Filamentous Actin-binding Protein Enriched in the Cell Cortex. J. Cell Biol. 1993, 120, 1417-1426.

15. Wu, H.; Reynolds, A.B.; Kanner, S.B.; Vines, R.R.; Parsons, J.T. Identification and characterization of a novel cytoskeleton-associated pp60src substrate. Mol. Cell. Biol. 1991, 11, 5113-5124.

16. Weed, S.A.; Du, Y.; Parsons, J.T. Translocation of cortactin to the cell periphery is mediated by the small GTPase Rac1. J. Cell Sci. 1998, 111, 2433-2443.

17. Siton, O.; Ideses, Y.; Albeck, S.; Unger, T.; Bershadsky, A.D.; Gov, N.S.; Bernheim-Groswasser, A. Cortactin releases the brakes in actin- based motility by enhancing WASP-VCA detachment from Arp2/3 branches. Curr. Biol. 2011, 21, 2092-2097.

18. Schuuring, E.; Verhoeven, E.; Litvinov, S.; Michalides, R.J.A.M. The product of the EMS1 gene, amplified and overexpressed in human carcinomas, is homologous to a v-src substrate and is located in cell-substratum contact sites. Mol. Cell. Biol. 1993, 13, 2891-2898. 
19. Schuuring, E.; Verhoeven, E.; Mooi, W.J.; Michalides, R.J.A.M. Identification and cloning of two overexpressed genes, U21B31/PRAD1 and EMS1, within the amplified chromosome 11q13 region in human carcinomas. Oncogene 1992, 7, 355-361.

20. Clark, E.S.; Brown, B.; Whigham, A.S.; Kochaishvili, A.; Yarbrough, W.G.; Weaver, A.M. Aggressiveness of HNSCC tumors depends on expression levels of cortactin, a gene in the 11q13 amplicon. Oncogene 2009, 28, 431-444.

21. Huang, C.; Liu, J.; Haudenschild, C.C.; Zhan, X. The Role of Tyrosine Phosphorylation of Cortactin in the Locomotion of Endothelial Cells. J. Biol. Chem. 1998, 273, 25770-25776.

22. Patel, A.S.; Schechter, G.L.; Wasilenko, W.J.; Somers, K.D. Overexpression of EMS1/cortactin in NIH3T3 fibroblasts causes increased cell motility and invasion in vitro. Oncogene 1998, 16, 3227-3232.

23. Kowalski, J.R.; Egile, C.; Gil, S.; Snapper, S.B.; Li, R.; Thomas, S.M. Cortactin regulates cell migration through activation of N-WASP. J. Cell Sci. 2005, 118, 79-87.

24. Rodrigo, J.P.; Garc1, L.A.; Ramos, S.; Lazo, P.S.; Sua, C. EMS1 gene amplification correlates with poor prognosis in squamous cell carcinomas of the head and neck. Clin. Cancer Res. 2000, 6, 3177-3182.

25. Rothschild, B.L.; Shim, A.H.; Ammer, A.G.; Kelley, L.C.; Irby, K.B.; Head, J.A.; Chen, L.; Varella-Garcia, M.; Sacks, P.G.; Frederick, B.; et al. Cortactin overexpression regulates actin-related protein $2 / 3$ complex activity, motility, and invasion in carcinomas with chromosome 11q13 amplification. Cancer Res. 2006, 66, 8017-8025.

26. Kelley, L.C.; Hayes, K.E.; Ammer, A.G.; Martin, K.H.; Weed, S.A. Cortactin phosphorylated by ERK1/2 localizes to sites of dynamic actin regulation and is required for carcinoma lamellipodia persistence. PLoS One 2010, 5, e13847.

27. Kelley, L.C.; Ammer, A.G.; Hayes, K.E.; Martin, K.H.; Machida, K.; Jia, L.; Mayer, B.J.; Weed, S.A. Oncogenic Src requires a wild-type counterpart to regulate invadopodia maturation. J. Cell Sci. 2010, 123, 3923-3932.

28. Luo, M.-L.; Shen, X.-M.; Zhang, Y.; Wei, F.; Xu, X.; Cai, Y.; Zhang, X.; Sun, Y.-T.; Zhan, Q.-M.; $\mathrm{Wu}, \mathrm{M}$; et al. Amplification and overexpression of CTTN (EMS1) contribute to the metastasis of esophageal squamous cell carcinoma by promoting cell migration and anoikis resistance. Cancer Res. 2006, 66, 11690-11699.

29. Li, Y.; Tondravi, M.; Liu, J.; Smith, E.; Haudenschild, C.C.; Kaczmarek, M.; Zhan, X. Cortactin potentiates bone metastasis of breast cancer cells. Cancer Res. 2001, 61, 6906-6911.

30. Mader, C.C.; Oser, M.; Magalhaes, M.A.O.; Bravo-Cordero, J.J.; Condeelis, J.; Koleske, A.J.; Gil-Henn, H. An EGFR-Src-Arg-Cortactin pathway mediates functional maturation of invadopodia and breast cancer cell invasion. Cancer Res. 2011, 71, 1730-1741.

31. Bowden, E.T.; Onikoyi, E.; Slack, R.; Myoui, A.; Yoneda, T.; Yamada, K.M.; Mueller, S.C. Co-localization of cortactin and phosphotyrosine identifies active invadopodia in human breast cancer. Exp. Cell Res. 2006, 321, 1240-1253.

32. Clark, E.S.; Weaver, A.M. A new role for cortactin in invadopodia: Regulation of protease secretion. Eur. J. Cell Biol. 2008, 87, 581-590.

33. Patel, B.P.; Shah, S.V.; Shukla, S.N.; Shah, P.M.; Patel, P.S. Clinical significance of MMP-2 and MMP-9 in patients with oral cancer. Head Neck 2007, 29, 564-572. 
34. Koontongkaew, S.; Amornphimoltham, P.; Monthanpisut, P.; Saensuk, T.; Leelakriangsak, M. Fibroblasts and extracellular matrix differently modulate MMP activation by primary and metastatic head and neck cancer cells. Med. Oncol. 2012, 29, 690-703.

35. Papaspyrou, K.; Brochhausen, C.; Schmidtmann, I.; Fruth, K.; Gouveris, H.; Kirckpatrick, J.; Mann, W.; Brieger, J. Fascin upregulation in primary head and neck squamous cell carcinoma is associated with lymphatic metastasis. Oncol. Lett. 2014, 7, 2041-2046.

36. Karasavvidou, F.; Barbanis, S.; Pappa, D.; Moutzouris, G.; Tzortzis, V.; Melekos, M.D.; Koukoulis, G. Fascin determination in urothelial carcinomas of the urinary bladder: A marker of invasiveness. Arch. Pathol. Lab. Med. 2008, 132, 1912-1915.

37. Adams, J.C. Roles of fascin in cell adhesion and motility. Curr. Opin. Cell Biol. 2004, 16, 590-596.

38. Jawhari, A.U.; Buda, A.; Jenkins, M.; Shehzad, K.; Sarraf, C.; Noda, M.; Farthing, M.J.G.; Pignatelli, M.; Adams, J.C. Fascin, an actin-bundling protein, modulates colonic epithelial cell invasiveness and differentiation in vitro. Am. J. Pathol. 2003, 162, 69-80.

39. Park, J.; Kim, J.-M.; Park, J.K.; Huang, S.; Kwak, S.Y.; Ryu, K.A.; Kong, G.; Park, J.; Koo, B.S. Association of p21-activated kinase-1 activity with aggressive tumor behavior and poor prognosis of head and neck cancer. Head Neck 2014, doi:10.1002/hed.23695.

40. McCarty, S.K.; Saji, M.; Zhang, X.; Jarjoura, D.; Fusco, A.; Vasko, V.V.; Ringel, M.D. Group I p21-activated kinases regulate thyroid cancer cell migration and are overexpressed and activated in thyroid cancer invasion. Endocr. Relat. Cancer 2010, 17, 989-999.

41. King, H.; Nicholas, N.S.; Wells, C.M. Role of p-21-activated kinases in cancer progression. Int. Rev. Cell Mol. Biol. 2014, 309, 347-387.

42. Dharmawardhane, S.; Brownson, D.; Lennartz, M.; Bokoch, G.M. Localization of p21-activated kinase 1 (PAK1) to pseudopodia, membrane ruffles, and phagocytic cups in activated human neutrophils. J. Leukoc. Biol. 1999, 66, 521-527.

43. Dharmawardhane, S.; Sanders, L.C.; Martin, S.S.; Daniels, R.H.; Bokoch, G.M. Localization of p21-activated kinase 1 (PAK1) to pinocytic vesicles and cortical actin structures in stimulated cells.

J. Cell Biol. 1997, 138, 1265-1278.

44. Delorme-Walker, V.D.; Peterson, J.R.; Chernoff, J.; Waterman, C.M.; Danuser, G.; DerMardirossian, C.; Bokoch, G.M. Pak1 regulates focal adhesion strength, myosin IIA distribution, and actin dynamics to optimize cell migration. J. Cell Biol. 2011, 193, 1289-1303.

45. Sanders, L.C. Inhibition of myosin light chain kinase by p21-activated kinase. Science 1999, 283, 2083-2085.

46. Stockton, R.A.; Schaefer, E.; Schwartz, M.A. p21-Activated kinase regulates endothelial permeability through modulation of contractility. J. Biol. Chem. 2004, 279, 46621-46630.

47. Arber, S.; Barbayannis, F.A.; Hanser, H.; Schneider, C.; Stanyon, C.A.; Bernard, O.; Caroni, P. Regulation of actin dynamics through phosphorylation of cofilin by LIM-kinase. Nature 1998, 393, 805-809.

48. Yang, N.; Higuchi, O.; Ohashi, K.; Nagata, K.; Wada, A.; Kangawa, K.; Nishida, E.; Mizuno, K. Cofilin phosphorylation by LIM-kinase 1 and its role in Rac-mediated actin reorganization. Nature 1998, 393, 809-812.

49. Kelley, L.C.; Shahab, S.; Weed, S.A. Actin cytoskeletal mediators of motility and invasion amplified and overexpressed in head and neck cancer. Clin. Exp. Metastasis 2008, 25, 289-304. 
50. Vadlamudi, R.K.; Li, F.; Barnes, C.J.; Bagheri-Yarmand, R.; Kumar, R. P41-Arc subunit of human Arp2/3 complex is a p21-activated kinase-1-interacting substrate. EMBO Rep. 2004, 5, 154-160.

51. Webb, B.A.; Zhou, S.; Eves, R.; Shen, L.; Jia, L.; Mak, A.S. Phosphorylation of cortactin by p21-activated kinase. Arch. Biochem. Biophys. 2006, 456, 183-193.

52. Elloul, S.; Vaksman, O.; Stavnes, H.T.; Trope, C.G.; Davidson, B.; Reich, R. Mesenchymal-toepithelial transition determinants as characteristics of ovarian carcinoma effusions. Clin. Exp. Metastasis 2010, 27, 161-172.

53. Goc, A.; Al-Azayzih, A.; Abdalla, M.; Al-Husein, B.; Kavuri, S.; Lee, J.; Moses, K.; Somanath, P.R. P21 activated kinase-1 (Pak1) promotes prostate tumor growth and microinvasion via inhibition of transforming growth factor $\beta$ expression and enhanced matrix metalloproteinase 9 secretion. J. Biol. Chem. 2013, 288, 3025-3035.

54. Rider, L.; Oladimeji, P.; Diakonova, M. PAK1 regulates breast cancer cell invasion through secretion of matrix metalloproteinases in response to prolactin and three-dimensional collagen IV. Mol. Endocrinol. 2013, 27, 1048-1064.

55. Srikrishna, G. S100A8 and S100A9: New insights into their roles in malignancy. J. Innate Immun. 2012, 4, 31-40.

56. Donato, R. S100: A multigenic family of calcium-modulated proteins of the EF-hand type with intracellular and extracellular functional roles. Int. J. Biochem. Cell Biol. 2001, 33, 637-668.

57. Heizmann, C.W.; Fritz, G.; Schäfer, B.W. S100 proteins: Structure, functions and pathology. Front. Biosci. 2002, 7, d1356-d1368.

58. Leukert, N.; Vogl, T.; Strupat, K.; Reichelt, R.; Sorg, C.; Roth, J. Calcium-dependent tetramer formation of S100A8 and S100A9 is essential for biological activity. J. Mol. Biol. 2006, 359, 961-972.

59. Silva, E.J.; Argyris, P.; Zou, X.; Ross, K.F.; Herzberg, M.C. S100A8/A9 regulates MMP-2 expression and invasion and migration by carcinoma cells. Int. J. Biochem. Cell Biol. 2014, 55, 279-287.

60. Fung, L.F.; Lo, A.K.F.; Yuen, P.W.; Wang, X.H.; Tsao, S.W. Differential gene expression in nasopharyngeal carcinoma cells. Life Sci. 2000, 67, 923-936.

61. Ghavami, S.; Chitayat, S.; Hashemi, M.; Eshraghi, M.; Chazin, W.J.; Halayko, A.J.; Kerkhoff, C. S100A8/A9: A Janus-faced molecule in cancer therapy and tumorgenesis. Eur. J. Pharmacol. 2009, 625, 73-83.

62. Fogh, B.S.; Multhaupt, H.A.B.; Couchman, J.R. Protein kinase C, focal adhesions and the regulation of cell migration. J. Histochem. Cytochem. 2014, 62, 172-184.

63. Zaidel-Bar, R.; Itzkovitz, S.; Ma'ayan, A.; Iyengar, R.; Geiger, B. Functional atlas of the integrin adhesome. Nat. Cell Biol. 2007, 9, 858-867.

64. Zaidel-Bar, R.; Cohen, M.; Addadi, L.; Geiger, B. Hierarchical assembly of cell-matrix adhesion complexes. Biochem. Soc. Trans. 2004, 32, 416-420.

65. Tang, H.; Li, A.; Bi, J.; Veltman, D.M.; Zech, T.; Spence, H.J.; Yu, X.; Timpson, P.; Insall, R.H.; Frame, M.C.; et al. Loss of Scar/WAVE complex promotes N-WASP- and FAK-dependent invasion. Curr. Biol. 2013, 23, 107-117.

66. Schlaepfer, D.D.; Mitra, S.K. Multiple connections link FAK to cell motility and invasion. Curr. Opin. Genet. Dev. 2004, 14, 92-101. 
67. Canel, M.; Secades, P.; Rodrigo, J.-P.; Cabanillas, R.; Herrero, A.; Suarez, C.; Chiara, M.-D. Overexpression of focal adhesion kinase in head and neck squamous cell carcinoma is independent of fak gene copy number. Clin. Cancer Res. 2006, 12, 3272-3279.

68. Gabarra-Niecko, V.; Schaller, M.D.; Dunty, J.M. FAK regulates biological processes important for the pathogenesis of cancer. Cancer Metastasis Rev. 2003, 22, 359-374.

69. Hsia, D.A.; Mitra, S.K.; Hauck, C.R.; Streblow, D.N.; Nelson, J.A.; Ilic, D.; Huang, S.; Li, E.; Nemerow, G.R.; Leng, J.; et al. Differential regulation of cell motility and invasion by FAK. J. Cell Biol. 2003, 160, 753-767.

70. McLean, G.W.; Carragher, N.O.; Avizienyte, E.; Evans, J.; Brunton, V.G.; Frame, M.C. The role of focal-adhesion kinase in cancer-A new therapeutic opportunity. Nat. Rev. Cancer 2005, 5, 505-515.

71. Schaller, M.D.; Borgman, C.A.; Cobb, B.S.; Vines, R.R.; Reynolds, A.B.; Parsons, J.T. pp125FAK a structurally distinctive protein-tyrosine kinase associated with focal adhesions. Proc. Natl. Acad. Sci. USA 1992, 89, 5192-5196.

72. Shanthi, E.; Krishna, M.H.; Arunesh, G.M.; Venkateswara Reddy, K.; Sooriya Kumar, J.; Viswanadhan, V.N. Focal adhesion kinase inhibitors in the treatment of metastatic cancer: A patent review. Expert Opin. Ther. Pat. 2014, 24, 1077-1100.

73. Dao, P.; Smith, N.; Tomkiewicz-Raulet, C.; Yen-Pon, E.; Camacho-Artacho, M.; Lietha, D.; Herbeuval, J.-P.; Coumoul, X.; Garbay, C.; Chen, H. Design, synthesis, and evaluation of novel imidazo(1,2-a)(1,3,5)triazines and their derivatives as focal adhesion kinase inhibitors with antitumor activity. J. Med. Chem. 2014, 58, 237-251.

74. Lin, J.-J.; Su, J.-H.; Tsai, C.-C.; Chen, Y.-J.; Liao, M.-H.; Wu, Y.-J. 11-epi-Sinulariolide acetate reduces cell migration and invasion of human hepatocellular carcinoma by reducing the activation of ERK1/2, p38MAPK and FAK/PI3K/AKT/mTOR signaling pathways. Mar. Drugs 2014, 12, 4783-4798.

75. Guo, B.; Su, J.; Zhang, T.; Wang, K.; Li, X. Fangchinoline as a kinase inhibitor targets FAK and suppresses FAK-mediated signaling pathway in A549. J. Drug Target. 2014, 24, 1-9.

76. Steed, P.M.; Chow, A.H. Intracellular signaling by phospholipase D as a therapeutic target. Curr. Pharm. Biotechnol. 2001, 2, 241-256.

77. Jenkins, G.M.; Frohman, M.A. Phospholipase D: A lipid centric review. Cell. Mol. Life Sci. 2005, 62, 2305-2316.

78. Kim, J.H.; Kim, H.; Jeon, H.; Suh, P.-G.; Ryu, S.H. Phospholipase D1 regulates cell migration in a lipase activity-independent manner. J. Biol. Chem. 2006, 281, 15747-15756.

79. Ahn, B.-H.; Kim, S.Y.; Kim, E.H.; Choi, K.S.; Kwon, T.K.; Lee, Y.H.; Chang, J.-S.; Kim, M.-S.; Jo, Y.-H.; Min, D.S. Transmodulation between phospholipase D and c-Src enhances cell proliferation. Mol. Cell. Biol. 2003, 23, 3103-3115.

80. Martinez-quiles, N.; Ho, H.H.; Kirschner, M.W.; Ramesh, N.; Geha, R.S. Erk/Src phosphorylation of cortactin acts as a switch on-switch off mechanism that controls its ability to activate N-WASP. Mol. Cell. Biol. 2004, 24, 5269-5280.

81. Noh, D.Y.; Ahn, S.J.; Lee, R.A.; Park, I.A.; Kim, J.H.; Suh, P.G.; Ryu, S.H.; Lee, K.H.; Han, J.S. Overexpression of phospholipase D1 in human breast cancer tissues. Cancer Lett. 2000, 161, 207-214.

82. Kelley, L.C.; Hayes, K.E.; Ammer, A.G.; Martin, K.H.; Weed, S.A. Revisiting the ERK/Src cortactin switch. Commun. Integr. Biol. 2011, 4, 205-207. 
83. O’Reilly, M.C.; Scott, S.A.; Brown, K.A.; Oguin, T.H.; Thomas, P.G.; Daniels, J.S.; Morrison, R.; Brown, H.A.; Lindsley, C.W. Development of dual PLD1/2 and PLD2 selective inhibitors from a common 1,3,8-Triazaspiro(4.5)decane Core: Discovery of M1298 and M1299 that decrease invasive migration in U87-MG glioblastoma cells. J. Med. Chem. 2013, 56, 2695-2699.

84. Park, M.H.; Min, D.S. Quercetin-induced downregulation of phospholipase D1 inhibits proliferation and invasion in U87 glioma cells. Biochem. Biophys. Res. Commun. 2011, 412, 710-715.

85. Simpson, D.R.; Mell, L.K.; Cohen, E.E.W. Targeting the PI3K/AKT/mTOR pathway in squamous cell carcinoma of the head and neck. Oral Oncol. 2014, in press.

86. Lui, V.W.Y.; Hedberg, M.L.; Li, H.; Vangara, B.S.; Pendleton, K.; Zeng, Y.; Lu, Y.; Zhang, Q.; Du, Y.; Gilbert, B.R.; et al. Frequent mutation of the PI3K pathway in head and neck cancer defines predictive biomarkers. Cancer Discov. 2013, 3, 761-769.

87. Engelman, J.A.; Luo, J.; Cantley, L.C. The evolution of phosphatidylinositol 3-kinases as regulators of growth and metabolism. Nat. Rev. Genet. 2006, 7, 606-619.

88. Volinia, S.; Hiles, I.; Ormondroyd, E.; Nizetic, D.; Antonacci, R.; Rocchi, M.; Waterfield, M.D. Molecular cloning, cDNA sequence, and chromosomal localization of the human phosphatidylinositol 3-kinase p110 alpha (PIK3CA) gene. Genomics 1994, 24, 472-477.

89. Roymans, D.; Slegers, H. Phosphatidylinositol 3-kinases in tumor progression. Eur. J. Biochem. 2001, 268, 487-498.

90. Estilo, C.L.; O-Charoenrat, P.; Ngai, I.; Patel, S.G.; Reddy, P.G.; Dao, S.; Shaha, A.R.; Kraus, D.H.; Boyle, J.O.; Wong, R.J.; et al. The role of novel oncogenes squamous cell carcinoma-related oncogene and phosphatidylinositol 3-kinase p110alpha in squamous cell carcinoma of the oral tongue. Clin. Cancer Res. 2003, 9, 2300-2306.

91. Katoh, M. WNT and FGF gene clusters. Int. J. Oncol. 2002, 21, 1269-1273.

92. Marshall, M.E.; Hinz, T.K.; Kono, S.A.; Singleton, K.R.; Bichon, B.; Ware, K.E.; Marek, L.; Frederick, B.A.; Raben, D.; Heasley, L.E. Fibroblast growth factor receptors are components of autocrine signaling networks in head and neck squamous cell carcinoma cells. Clin. Cancer Res. 2011, 17, 5016-5025.

93. Li, W.; Wang, C.; Juhn, S.K.; Ondrey, F.G.; Lin, J. Expression of fibroblast growth factor binding protein in head and neck cancer. Arch. Otolaryngol. Head Neck Surg. 2009, 135, 896-901.

94. Friedl, P.; Locker, J.; Sahai, E.; Segall, J.E. Classifying collective cancer cell invasion. Nat. Cell Biol. 2012, 14, 777-783.

95. Friedl, P.; Alexander, S. Cancer invasion and the microenvironment: Plasticity and reciprocity. Cell 2011, 147, 992-1009.

96. Mandal, M.; Myers, J.N.; Lippman, S.M.; Johnson, F.M.; Williams, M.D.; Rayala, S.; Ohshiro, K.; Rosenthal, D.I.; Weber, R.S.; Gallick, G.E.; et al. Epithelial to mesenchymal transition in head and neck squamous carcinoma: Association of Src activation with E-cadherin down-regulation, vimentin expression, and aggressive tumor features. Cancer 2008, 112, 2088-2100.

97. Sok, J.C.; Coppelli, F.M.; Thomas, S.M.; Lango, M.N.; Xi, S.; Hunt, J.L.; Freilino, M.L.; Graner, M.W.; Wikstrand, C.J.; Bigner, D.D.; et al. Mutant epidermal growth factor receptor (EGFRvIII) contributes to head and neck cancer growth and resistance to EGFR targeting. Clin. Cancer Res. 2006, 12, 5064-5073. 
98. Xi, S.; Zhang, Q.; Dyer, K.F.; Lerner, E.C.; Smithgall, T.E.; Gooding, W.E.; Kamens, J.; Grandis, J.R. Src kinases mediate STAT growth pathways in squamous cell carcinoma of the head and neck. J. Biol. Chem. 2003, 278, 31574-31583.

99. Bryne, M.; Koppang, H.S.; Lilleng, R.; Kjaerheim, A. Malignancy grading of the deep invasive margins of oral squamous cell carcinomas has high prognostic value. J. Pathol. 1992, 166, 375-381.

100. Basu, D.; Bewley, A.F.; Sperry, S.M.; Montone, K.T.; Gimotty, P.A.; Rasanen, K.; Facompre, N.D.; Weinstein, G.S.; Nakagawa, H.; Diehl, J.A.; et al. EGFR inhibition promotes an aggressive invasion pattern mediated by mesenchymal-like tumor cells within squamous cell carcinomas. Mol. Cancer Ther. 2013, 12, 2176-2186.

101. Basu, D.; Montone, K.T.; Wang, L.-P.; Gimotty, P.A.; Hammond, R.; Diehl, J.A.; Rustgi, A.K.; Lee, J.T.; Rasanen, K.; Weinstein, G.S.; et al. Detecting and targeting mesenchymal-like subpopulations within squamous cell carcinomas. Cell Cycle 2011, 10, 2008-2016.

102. Basu, D.; Nguyen, T.-T.K.; Montone, K.T.; Zhang, G.; Wang, L.-P.; Diehl, J.A.; Rustgi, A.K.; Lee, J.T.; Weinstein, G.S.; Herlyn, M. Evidence for mesenchymal-like sub-populations within squamous cell carcinomas possessing chemoresistance and phenotypic plasticity. Oncogene 2010, 29, 4170-4182.

103. Kupferman, M.E.; Jiffar, T.; El-Naggar, A.; Yilmaz, T.; Zhou, G.; Xie, T.; Feng, L.; Wang, J.; Holsinger, F.C.; Yu, D.; et al. TrkB induces EMT and has a key role in invasion of head and neck squamous cell carcinoma. Oncogene 2010, 29, 2049-2059.

104. Sawatsubashi, M.; Yamada, T.; Fukushima, N.; Mizokami, H.; Tokunaga, O.; Shin, T. Association of vascular endothelial growth factor and mast cells with angiogenesis in laryngeal squamous cell carcinoma. Virchows Arch. 2000, 436, 243-248.

105. Ninck, S.; Reisser, C.; Dyckhoff, G.; Helmke, B.; Bauer, H.; Herold-Mende, C. Expression profiles of angiogenic growth factors in squamous cell carcinomas of the head and neck. Int. J. Cancer 2003, 106, 34-44.

106. Liss, C.; Fekete, M.J.; Hasina, R.; Lam, C.D.; Lingen, M.W. Paracrine angiogenic loop between head-and-neck squamous-cell carcinomas and macrophages. Int. J. Cancer 2001, 93, 781-785.

107. Bran, B.; Bran, G.; Hormann, K.; Riedel, F. The platelet-derived growth factor receptor as a target for vascular endothelial growth factor-mediated anti-angiogeneic therapy in head and neck cancer. Int. J. Oncol. 2009, 34, 255-261.

108. Ali, M.A. Lymphatic microvessel density and the expression of lymphangiogenic factors in oral squamous cell carcinoma. Med. Princ. Pract. 2008, 17, 486-492.

109. Li, C.; Shintani, S.; Terakada, N.; Klosek, S.K.; Ishikawa, T.; Nakashiro, K.; Hamakawa, H. Microvessel density and expression of vascular endothelial growth factor, basic fibroblast growth factor, and platelet-derived endothelial growth factor in oral squamous cell carcinomas. Int. J. Oral Maxillofac. Surg. 2005, 34, 559-565.

110. Sedivy, R.; Beck-Mannagetta, J.; Haverkampf, C.; Battistutti, W.; Honigschnabl, S. Expression of vascular endothelial growth factor-C correlates with the lymphatic microvessel density and the nodal status in oral squamous cell cancer. J. Oral Pathol. Med. 2003, 32, 455-460.

111. Beasley, N.J.P.; Prevo, R.; Banerji, S.; Leek, R.D.; Moore, J.; van Trappen, P.; Cox, G.; Harris, A.L.; Jackson, D.G. Intratumoral lymphangiogenesis and lymph node metastasis in head and neck cancer. Cancer Res. 2002, 62, 1315-1320. 
112. Jatana, K.R.; Balasubramanian, P.; Lang, J.C.; Yang, L.; Jatana, C.; White, E.; Agrawal, A.; Ozer, E.; Schuller, D.E.; Teknos, T.N.; et al. Significance of cirulating tumors cells in patients with squamous cell carcinoma of the head and neck. Arch. Otolaryngol. Head Neck Surg. 2010, 136, 1274-1279.

113. Buglione, M.; Grisanti, S.; Almici, C.; Mangoni, M.; Polli, C.; Consoli, F.; Verardi, R.; Costa, L.; Paiar, F.; Pasinetti, N.; et al. Circulating tumor cells in locally advanced cancer: Preliminary report about their possible role in predicting response to non-surgical treatment and survival. Eur. J. Cancer 2012, 48, 3019-3026.

114. Krishnamurthy, S.; Warner, K.A.; Dong, Z.; Imai, A.; Nör, C.; Ward, B.B.; Helman, J.I.; Taichman, R.S.; Bellile, E.L.; McCauley, L.K.; et al. Endothelial interleukin-6 defines the tumorigenic potential of primary human cancer stem cells. Stem Cells 2014, 32, 2845-2857.

115. Nör, C.; Zhang, Z.; Warner, K.A.; Bernardi, L.; Visioli, F.; Helman, J.I.; Roesler, R.; Nör, J.E. Cisplatin induces Bmi-1 and enhances the stem cell fraction in head and neck cancer. Neoplasia 2014, 16, 137-146.

116. Zuo, J.-H.; Zhu, W.; Li, M.-Y.; Li, X.-H.; Yi, H.; Zeng, G.-Q.; Wan, X.-X.; He, Q.-Y.; Li, J.-H.; $\mathrm{Qu}, \mathrm{J} . \mathrm{Q}$.; et al. Activation of EGFR promotes squamous carcinoma SCC10A cell migration and invasion via inducing EMT-like phenotype change and MMP-9-mediated degradation of E-cadherin. J. Cell. Biochem. 2011, 112, 2508-2517.

117. St. John, M.A.; Dohadwala, M.; Luo, J.; Wang, G.; Lee, G.; Shih, H.; Heinrich, E.; Krysan, K.; Walser, T.; Hazra, S.; et al. Proinflammatory mediators upregulate snail in head and neck squamous cell carcinoma. Clin. Cancer Res. 2009, 15, 6018-6027.

118. Cao, X.-L.; Xu, R.-J.; Zheng, Y.-Y.; Liu, J.; Teng, Y.-S.; Li, Y.; Zhu, J. Expression of type IV collagen, metalloproteinase-2, metalloproteinase-9 and tissue inhibitor of metalloproteinase-1 in laryngeal squamous cell carcinomas. Asian Pac. J. Cancer Prev. 2011, 12, 3245-3249.

119. Nielsen, J.D.; Moeslund, M.; Wandall, H.H.; Dabelsteen, S. Influences of tumor stroma on the malignant phenotype. J. Oral Pathol. Med. 2008, 37, 412-416.

120. Parikka, M.; Nissienen, L.; Kainulainen, T.; Bruckner-Tuderman, L.; Salo, T.; Heino, J.; Tasanen, K. Collagen XVII promotes integrin-mediated squamous cell carcinoma transmigration-A novel role for $\alpha$ IIb integrin and tirofiban. Exp. Cell Res. 2006, 312, 1431-1438.

121. Prussin, C.; Metcalfe, D.D. IgE, mast cells, basophils, and eosinophils. J. Allergy Clin. Imunol. 2003, 111, S486-S494.

122. Barth, P.J.; Schweinsberg zu Schweinsberg, T.; Ramaswamy, A.; Moll, R. CD34 $4^{+}$fibrocytes, alpha-smooth muscle antigen-positive myofibroblasts, and CD117 expression in the stroma of invasive squamous cell carcinomas of the oral cavity, pharynx, and larynx. Virchows Arch. 2004, 444, 231-234.

123. Iamaroon, A.; Pongsiriwet, S.; Jittidecharaks, S.; Pattanaporn, K.; Prapayasatok, S.; Wanachantararak, S. Increase of mast cells and tumor angiogenesis in oral squamous cell carcinoma. J. Oral Pathol. Med. 2003, 32, 195-199.

124. Beckhove, P.; Helmke, B.M.; Ziouta, Y.; Bucur, M.; Borner, W.; Mogler, C.; Dyckhoff, G.; Herold-Mende, C. Heparanase expression at the invasion front of human head and neck cancers and correlation with poor prognosis. Clin. Cancer Res. 2005, 11, 2899-2906. 
125. Friedmann, Y.; Vlodavsky, I.; Aingorn, H.; Aviv, A.; Peretz, T.; Pecker, I.; Pappo, O. Expression of heparanase in normal, dysplastic and neoplastic human colonic mucosa and stroma. Am. J. Pathol. 2000, 157, 1167-1175.

126. Nathan, C. Neutrophils and immunity: Challenges and opportunities. Nat. Rev. Immunol. 2006, 6, $173-182$.

127. Waugh, D.J.J.; Wilson, C. The interleukin-8 pathway in cancer. Clin. Cancer Res. 2008, 14, 6735-6741.

128. Scapini, P.; Bazzoni, F.; Cassatella, M.A. Regulation of B-cell-activating factor (BAFF)/B lymphocyte stimulator (BLyS) expression in human neutrophils. Immunol. Lett. 2008, 116, 1-6.

129. Scapini, P.; Morini, M.; Tecchio, C.; Minghelli, S.; di Carlo, E.; Tanghetti, E.; Albini, A.; Lowell, C.; Berton, G.; Noonan, D.M.; et al. CXCL1/Macrophage inflammatory protein-2-induced angiogenesis in vivo is mediated by neutrophil-derived vascular endothelial growth factor-A. J. Immunol. 2004, 172, 5034-5040.

130. Benelli, R.; Morini, M.; Carrozzino, F.; Ferrari, N.; Minghelli, S.; Santi, L.; Cassatella, M.; Noonan, D.M.; Albini, A. Neutrophils as a key cellular target for angiostatin: Implications for regulation of angiogenesis and inflammation. FASEB J. 2002, 2, 267-269.

131. Eming, S.A.; Krieg, T.; Davidson, J.M. Inflammation in wound repair: Molecular and cellular mechanisms. J. Investig. Dermatol. 2007, 127, 514-525.

132. Li, C.; Shintani, S.; Terakada, N.; Nakashiro, K.; Hamakawa, H. Infiltration of tumor-associated macrophages in human oral squamous cell carcinoma. Oncol. Rep. 2002, 9, 1219-1223.

133. Marcus, B.; Arenberg, D.; Lee, J.; Kleer, C.; Chepeha, D.B.; Schmalbach, C.E.; Islam, M.; Paul, S.; Pan, Q.; Hanash, S.; et al. Prognostic factors in oral cavity and oropharyngeal squamous cell carcinoma. Cancer 2004, 101, 2779-2787.

134. Liu, S.-Y.; Chang, L.-C.; Pan, L.-F.; Hung, Y.-J.; Lee, C.-H.; Shieh, Y.-S. Clinicopathologic significance of tumor cell-lined vessel and microenvironment in oral squamous cell carcinoma. Oral Oncol. 2008, 44, 277-285.

135. Yamaguchi, H.; Pixley, F.; Condeelis, J. Invadopodia and podosomes in tumor invasion. Eur. J. Cell Biol. 2006, 85, 213-218.

136. Wyckoff, J.; Wang, W.; Lin, E.Y.; Wang, Y.; Pixley, F.; Stanley, E.R.; Graf, T.; Pollard, J.W.; Segall, J.; Condeelis, J. A paracrine loop between tumor cells and macrophages is required for tumor cell migration in mammary tumors. Cancer Res. 2004, 64, 7022-7029.

137. Goswami, S.; Sahai, E.; Wyckoff, J.; Cammer, M.; Cox, D.; Pixley, F.J.; Stanley, E.R.; Segall, J.E.; Condeelis, J.S. Macrophages promote the invasion of breast carcinoma cells via a colony-stimulating factor-1/epidermal growth factor paracrine loop. Cancer Res. 2005, 65, 5278-5283.

138. Dumitru, C.A.; Gholaman, H.; Trellakis, S.; Bruderek, K.; Dominas, N.; Gu, X.; Bankfalvi, A.; Whiteside, T.L.; Lang, S.; Brandau, S. Tumor-derived macrophage migration inhibitory factor modulates the biology of head and neck cancer cells via neutrophil activation. Int. J. Cancer 2011, $129,859-869$.

139. Linder, S.; Nelson, D.; Weiss, M.; Aepfelbacher, M. Wiskott-Aldrich syndrome protein regulates podosomes in primary human macrophages. Proc. Natl. Acad. Sci. USA 1999, 96, 9648-9653.

140. Schachtner, H.; Calaminus, S.D.J.; Thomas, S.G.; Machesky, L.M. Podosomes in adhesion, migration, mechanosensing and matrix remodeling. Cytoskeleton 2013, 70, 572-589. 
141. Varon, C.; Tatin, F.; Moreau, V.; van Obberghen-Schilling, E.; Fernandez-Sauze, S.; Reuzeau, E.; Kramer, I.; Genot, E. Transforming growth factor beta induces rosettes of podosomes in primary aortic endothelial cells. Mol. Cell. Biol. 2006, 26, 3582-3594.

142. Chang, K.-P.; Kao, H.-K.; Yen, T.-C.; Chang, Y.-L.; Liang, Y.; Liu, S.-C.; Lee, L.-Y.; Chang, Y.-L.; Kang, C.-J.; Chen, I.-H.; et al. Overexpression of macrophage inflammatory protein-3alpha in oral cavity squamous cell carcinoma is associated with nodal metastasis. Oral Oncol. 2011, 47, 108-113.

143. Osiak, A.-E.; Zenner, G.; Linder, S. Subconfluent endothelial cells form podosomes downstream of cytokine and RhoGTPase signaling. Exp. Cell Res. 2005, 307, 342-353.

144. Yamaguchi, H.; Lorenz, M.; Kempiak, S.; Sarmieto, C.; Coniglio, S.; Symons, M.; Segall, J.; Eddy, R.; Miki, H.; Takenawa, T.; et al. Molecular mechanisms of invadopodium formation: The role of the N-WASP-Arp2/3 complex pathway and cofilin. J. Cell Biol. 2005, 168, 441-452.

145. Mizutani, K.; Miki, H.; He, H.; Maruta, H.; Takenawa, T. Essential role of neural Wiskot-Aldrich syndrome protein in podosome formation and degradation of extracellular matrix in src-transformed fibroblasts. Cancer Res. 2002, 62, 669-674.

146. Spinardi, L.; Rietdorf, J.; Nitsch, L.; Bono, M.; Tacchetti, C.; Way, M.; Marchisio, P.C. A dynamic podosome-like structure of endothelial cells. Exp. Cell Res. 2004, 295, 360-374.

147. Zeng, Q.; Li, S.; Chepeha, D.B.; Giordano, T.J.; Li, J.; Zhang, H.; Polverini, P.J.; Nor, J.; Kitajewski, J.; Wang, C.-Y. Crosstalk between tumor and endothelial cells promotes tumor angiogenesis by MAPK activation of Notch signaling. Cancer Cell 2005, 8, 13-23.

148. Goetz, J.G.; Minguet, S.; Navarro-Lerida, I.; Lazcano, J.J.; Samaniego, R.; Calvo, E.; Tello, M.; Osteso-Ibanez, T.; Pellinen, T.; Echarri, A.; et al. Biomechanical remodeling of the microenvironment by stromal caveolin-1 favors tumor invasion and metastasis. Cell 2011, 146, 148-163.

149. Gaggioli, C.; Hooper, S.; Hidalgo-Carcedo, C.; Grosse, R.; Marshall, J.F.; Harrington, K.; Sahai, E. Fibroblast-led collective invasion of carcinoma cells with differing roles for RhoGTPases in leading and following cells. Nat. Cell Biol. 2007, 9, 1392-1400.

150. Kelly, T.; Mueller, S.C.; Yeh, Y.; Chen, W.T. Invadopodia promote proteolysis of a wide variety of extracellular matrix proteins. J. Cell. Physiol. 1994, 158, 299-308.

151. Lim, K.P.; Cirillo, N.; Hassona, Y.; Wei, W.; Thurlow, J.K.; Cheong, S.C.; Pitiyage, G.; Parkinson, E.K.; Prime, S.S. Fibroblast gene expression profile reflects the stage of tumour progression in oral squamous cell carcinoma. J. Pathol. 2011, 223, 459-469.

152. Abram, C.L.; Seals, D.F.; Pass, I.; Salinsky, D.; Maurer, L.; Roth, T.M.; Courtneidge, S.A. The adaptor protein fish associates with members of the ADAMs family and localizes to podosomes of Src-transformed cells. J. Biol. Chem. 2003, 278, 16844-16851.

153. Seals, D.F.; Azucena, E.F.J.; Pass, I.; Tesfay, L.; Gordon, R.; Woodrow, M.; Resau, J.H.; Courtneidge, S.A. The adaptor protein Tks5/Fish is required for podosome formation and function, and for the protease-driven invasion of cancer cells. Cancer Cell 2005, 7, 155-165.

154. Hinsley, E.E.; Hunt, S.; Hunter, K.D.; Whawell, S.A.; Lambert, D.W. Endothelin-1 stimulates motility of head and neck squamous carcinoma cells by promoting stromal-epithelial interactions. Int. J. Cancer 2012, 130, 40-47.

155. Ishikawa, T.; Nakashiro, K.; Klosek, S.K.; Goda, H.; Hara, S.; Uchida, D.; Hamakawa, H. Hypoxia enhances CXCR4 expression by activating HIF-1 in oral squamous cell carcinoma. Oncol. Rep. 2009, 21, 707-712. 
156. Uchida, D.; Begum, N.M.; Almofti, A.; Nakashiro, K.; Kawamata, H.; Tateishi, Y.; Hamakawa, H.; Yoshida, H.; Sato, M. Possible role of stromal-cell-derived factor-1/CXCR4 signaling on lymph node metastasis of oral squamous cell carcinoma. Exp. Cell Res. 2003, 290, 289-302.

157. Chuma, M.; Sakamoto, M.; Yasuda, J.; Fujii, G.; Nakanishi, K.; Tsuchiya, A.; Asaka, M.; Hirohashi, S. Overexpression of cortactin is involved in motility and metastasis of hepatocellular carcinoma. J. Hepatol. 2004, 41, 629-636.

158. Liu, Y.; Hu, T.; Shen, J.; Li, S.F.; Lin, J.W.; Zheng, X.H.; Gao, Q.H.; Zhou, H.M. Separation, cultivation and biological characteristics of oral carcinoma-associated fibroblasts. Oral Dis. 2006, $12,375-380$.

159. Lewis, M.P.; Lygoe, K.A.; Nystrom, M.L.; Anderson, W.P.; Speight, P.M.; Marshall, J.F.; Thomas, G.J. Tumor-derived TGF- $\beta 1$ modulates myofibroblast differentiation and promotes HGF/SF-dependent invasion of squamous carcinoma cells. Br. J. Cancer 2004, 90, 822-832.

160. Marsh, D.; Suchak, K.; Moutasim, K.A.; Vallath, S.; Hopper, C.; Jerjes, W.; Upile, T.; Kalavrezos, N.; Violette, S.M.; Weinreb, P.H.; et al. Stromal features are predictive of disease mortality in oral cancer patients. J. Pathol. 2011, 223, 470-481.

161. Wheeler, S.E.; Shi, H.; Lin, F.; Dasari, S.; Bednash, J.; Thorne, S.; Watkins, S.; Joshi, R.; Thomas, S.M. Enhancement of head and neck squamous cell carcinoma proliferation, invasion, and metastasis by tumor-associated fibroblasts in preclinical models. Head Neck 2014, 36, 385-392.

162. Taddei, M.L.; Cavallini, L.; Comito, G.; Giannoni, E.; Folini, M.; Marini, A.; Gandellini, P.; Morandi, A.; Pintus, G.; Raspollini, M.R.; et al. Senescent stroma promotes prostate cancer progression: The role of miR-210. Mol. Oncol. 2014, 8, 1729-1746.

163. Hassona, Y.; Cirillo, N.; Heesom, K.; Parkinson, E.K.; Prime, S.S. Senescent cancer-associated fibroblasts secrete active MMP-2 that promotes keratinocyte dis-cohesion and invasion. Br. J. Cancer 2014, 111, 1230-1237.

164. Hassona, Y.; Cirillo, N.; Lim, K.P.; Herman, A.; Mellone, M.; Thomas, G.J.; Pitiyage, G.N.; Parkinson, E.K.; Prime, S.S. Progression of genotype-specific oral cancer leads to senescence of cancer-associated fibroblasts and is mediated by oxidative stress and TGF- $\beta$. Carcinogenesis 2013, $34,1286-1295$.

165. Ren, C.; Cheng, X.; Lu, B.; Yang, G. Activation of interleukin-6/signal transducer and activator of transcription 3 by human papillomavirus early proteins 6 induces fibroblast senescence to promote cervical tumourigenesis through autocrine and paracrine pathways in tumour microenvironment. Eur. J. Cancer 2013, 49, 3889-3899.

166. Capparelli, C.; Chiavarina, B.; Whitaker-Menezes, D.; Pestell, T.G.; Pestell, R.G.; Hulit, J.; Andò, S.; Howell, A.; Martinez-Outschoorn, U.E.; Sotgia, F.; et al. CDK inhibitors (p16/p19/p21) induce senescence and autophagy in cancer-associated fibroblasts, "fueling" tumor growth via paracrine interactions, without an increase in neo-angiogenesis. Cell Cycle 2012, 11, 3599-3610.

167. Capparelli, C.; Whitaker-Menezes, D.; Guido, C.; Balliet, R.; Pestell, T.G.; Howell, A.; Sneddon, S.; Pestell, R.G.; Martinez-Outschoorn, U.; Lisanti, M.P.; et al. CTGF drives autophagy, glycolysis and senescence in cancer-associated fibroblasts via HIF1 activation, metabolically promoting tumor growth. Cell Cycle 2012, 11, 2272-2284. 
168. Ammer, A.G.; Kelley, L.C.; Hayes, K.E.; Evans, J.V.; Lopez-skinner, A.; Martin, K.H.; Frederick, B.; Rothschild, B.L.; Elvin, P.; Green, T.P.; et al. Saracatinib impairs head and neck squamous cell carcinoma invasion by disruption invadopodia function. J. Cancer Sci. Ther. 2009, 1, 52-61.

169. Nozawa, H.; Howell, G.; Suzuki, S.; Zhang, Q.; Qi, Y.; Klein-Seetharaman, J.; Wells, A.; Grandis, J.R.; Thomas, S.M. Combined inhibition of PLC $\gamma$-1 and c-Src abrogates epidermal growth factor receptor-mediated head and neck squamous cell carcinoma invasion. Clin. Cancer Res. 2008, 14, 4336-4344.

170. Koppikar, P.; Choi, S.-H.; Egloff, A.M.; Cai, Q.; Suzuki, S.; Freilino, M.; Nozawa, H.; Thomas, S.M.; Gooding, W.E.; Siegfried, J.M.; et al. Combined inhibition of c-Src and epidermal growth factor receptor abrogates growth and invasion of head and neck squamous cell carcinoma. Clin. Cancer Res. 2008, 14, 4284-4291.

171. Fury, M.G.; Baxi, S.; Shen, R.; Kelly, K.W.; Lipson, B.L.; Carlson, D.; Stambuk, H.; Haque, S.; Pfister, D.G. Phase II study of saracatinib (AZD0530) for patients with recurrent or metastatic head and neck squamous cell carcinoma (HNSCC). Anticancer Res. 2011, 31, 249-253.

172. Johnson, F.M.; Saigal, B.; Talpaz, M.; Donato, N.J. Dasatinib (BMS-354825) tyrosine kinase inhibitor suppresses invasion and induces cell cycle arrest and apoptosis of head and neck squamous cell carcinoma and non-small cell lung cancer cells. Clin. Cancer Res. 2005, 11, 6924-6932.

173. Brooks, H.D.; Glisson, B.S.; Bekele, B.N.; Johnson, F.M.; Ginsberg, L.E.; El-Naggar, A.; Culotta, K.S.; Takebe, N.; Wright, J.; Tran, H.T.; et al. Phase 2 study of dasatinib in the treatment of head and neck squamous cell carcinoma. Cancer 2011, 117, 2112-2119.

174. Srinivasan, D.; Plattner, R. Activation of Abl tyrosine kinases promotes invasion of aggressive breast cancer cells. Cancer Res. 2006, 66, 5648-5655.

175. Lin, J.; Sun, T.; Ji, L.; Deng, W.; Roth, J.; Minna, J.; Arlinghaus, R. Oncogenic activation of c-Abl in non-small cell lung cancer cells lacking FUS1 expression: Inhibition of c-Abl by the tumor suppressor gene product Fus1. Oncogene 2007, 26, 6989-6996.

176. Furlan, A.; Stagni, V.; Hussain, A.; Richelme, S.; Conti, F.; Prodosmo, A.; Destro, A.; Roncalli, M.; Barilà, D.; Maina, F. Abl interconnects oncogenic Met and p53 core pathways in cancer cells. Cell Death Differ. 2011, 18, 1608-1616.

177. Ganguly, S.S.; Fiore, L.S.; Sims, J.T.; Friend, J.W.; Srinivasan, D.; Thacker, M.A.; Cibull, M.L.; Wang, C.; Novak, M.; Kaetzel, D.M.; et al. C-Abl and Arg are activated in human primary melanomas, promote melanoma cell invasion via distinct pathways, and drive metastatic progression. Oncogene 2012, 31, 1804-1816.

178. Smith-Pearson, P.S.; Greuber, E.K.; Yogalingam, G.; Pendergast, A.M. Abl kinases are required for invadopodia formation and chemokine-induced invasion. J. Biol. Chem. 2010, 285, 40201-40211.

179. Hayes, K.E.; Walk, E.L.; Ammer, A.G.; Kelley, L.C.; Martin, K.H.; Weed, S.A. Ableson kinases negatively regulate invadopodia function and invasion in head and neck squamous cell carcinoma by inhibiting an HB-EGF autocrine loop. Oncogene 2013, 32, 4766-4777.

180. Tsao, A.S.; Liu, S.; Fujimoto, J.; Wistuba, I.I.; Lee, J.J.; Marom, E.M.; Charnsangavej, C.; Fossella, F.V.; Tran, H.T.; Blumenschein, G.R.; et al. Phase II trials of imatinib mesylate and docetaxel in patients with metastatic non-small cell lung cancer and head and neck squamous cell carcinoma. J. Thorac. Oncol. 2011, 6, 2104-2111. 
181. Herbst, R.S.; Kim, E.S.; Harari, P.M. IMC-C225, an anti-epidermal growth factor receptor monoclonal antibody, for treatment of head and neck cancer. Expert Opin. Biol. Ther. 2001, 1, 719-732.

182. Huang, S.M.; Bock, J.M.; Harari, P.M. Epidermal growth factor receptor blockade with C225 modulates proliferation, apoptosis, and radiosensitivity in squamous cell carcinomas of the head and neck. Cancer Res. 1999, 59, 1935-1940.

183. Huang, S.M.; Harari, P.M. Modulation of radiation response after epidermal growth factor receptor blockade in squamous cell carcinomas: Inhibition of damage repair, cell cycle kinetics, and tumor angiogenesis. Clin. Cancer Res. 2000, 6, 2166-2174.

184. Fury, M.G.; Sherman, E.; Lisa, D.; Agarwal, N.; Algazy, K.; Brockstein, B.; Langer, C.; Lim, D.; Mehra, R.; Rajan, S.K.; et al. A randomized phase II study of cetuximab every 2 weeks at either 500 or $750 \mathrm{mg} / \mathrm{m} 2$ for patients with recurrent or metastatic head and neck squamous cell cancer. J. Natl. Compr. Canc. Netw. 2012, 10, 1391-1398.

185. Merlano, M.; Russi, E.; Benasso, M.; Corvò, R.; Colantonio, I.; Vigna-Taglianti, R.; Vigo, V.; Bacigalupo, A.; Numico, G.; Crosetto, N.; et al. Cisplatin-based chemoradiation plus cetuximab in locally advanced head and neck cancer: A phase II clinical study. Ann. Oncol. 2011, 22, 712-717.

186. Licitra, L.; Störkel, S.; Kerr, K.M.; van Cutsem, E.; Pirker, R.; Hirsch, F.R.; Vermorken, J.B.; von Heydebreck, A.; Esser, R.; Celik, I.; et al. Predictive value of epidermal growth factor receptor expression for first-line chemotherapy plus cetuximab in patients with head and neck and colorectal cancer: Analysis of data from the EXTREME and CRYSTAL studies. Eur. J. Cancer 2013, 49, 1161-1168.

187. Licitra, L.; Mesia, R.; Rivera, F.; Remenár, E.; Hitt, R.; Erfán, J.; Rottey, S.; Kawecki, A.; Zabolotnyy, D.; Benasso, M.; et al. Evaluation of EGFR gene copy number as a predictive biomarker for the efficacy of cetuximab in combination with chemotherapy in the first-line treatment of recurrent and/or metastatic squamous cell carcinoma of the head and neck: EXTREME study. Ann. Oncol. 2011, 22, 1078-1087.

188. Rivera, F.; García-Castaño, A.; Vega, N.; Vega-Villegas, M.E.; Gutiérrez-Sanz, L. Cetuximab in metastatic or recurrent head and neck cancer: The EXTREME trial. Expert Rev. Anticancer Ther. 2009, 9, 1421-1428.

189. William, W.N.; Kim, E.S.; Herbst, R.S. Cetuximab therapy for patients with advanced squamous cell carcinomas of the head and neck. Nat. Clin. Pract. Oncol. 2009, 6, 132-133.

190. Fuchs, D.; Heinold, A.; Opelz, G.; Daniel, V.; Naujokat, C. Salinomycin induces apoptosis and overcomes apoptosis resistance in human cancer cells. Biochem. Biophys. Res. Commun. 2009, 390, 743-749.

(C) 2015 by the authors; licensee MDPI, Basel, Switzerland. This article is an open access article distributed under the terms and conditions of the Creative Commons Attribution license (http://creativecommons.org/licenses/by/4.0/). 


\title{
Personal Information \\ Steven Michael Markwell
}

Permanent Address: 417 Timberline Apartments, Morgantown, WV 26505

Work Address: 1 Medical Center Drive, Weed Lab 1833 MBRCC, Morgantown, WV 26506

Cell phone: (304) 680-1897

Office: MBRCC 1833

Office phone: (304)293-3090

Preferred email address: smarkwell@mix.wvu.edu

Alternate email addresses: smarkwell@hsc.wvu.edu, smarkwe2@gmail.com

\section{Citizenship}

United States of America (USA)

\section{Education}

\author{
West Virginia University (August 2010-current) \\ Department of Biochemistry \\ Morgantown WV, USA \\ Doctor of Philosophy in Cancer Cell Biology \\ Expected graduation: December 2018
}

University of Illinois at Urbana - Champaign (August 2005-May 2009)

Department of Chemistry, School of Molecular and Cellular Biology

Urbana-Champaign Illinois, USA

Bachelor of Science in Chemistry, Molecular and Cellular Biology

Awarded May 2009

\section{Research Experience}

Research assistant under Scott Weed at West Virginia University, Morgantown WV, USA. Office phone: (304)293-3016. Janurary 2011 - current.

Research project focused on mechanisms of invasion and metastasis in squamous cell carcinoma of the head and neck.

Three dimensional organotypic invasion assay training under Claudia Andl at Vanderbilt University, Nashville TN, USA.

Dr. Andl is now at University of Central Florida.

Office phone: (407)823-1147.

July 2014 - August 2014. 1-week visitation.

In person training for generating organotypic invasion cultures and subsequent analysis modalities following the invasion period. 
Actin polymerization assay training and fluorometric analysis under Dorothy Schafer at University of Virginia, Charlottesville VA, USA. Office phone: (434)243-5297.

September 2013 - October 2013. 2-week visitation.

Hands on instruction in assembling actin filament networks, followed by assessment of mutant cortactin variants on actin assembly dynamics.

Senior thesis research under Alexander Scheeline at University of Illinois at Urbana, Champaign IL, USA. Office phone: (217)333-2999. January 2008 - May 2009.

Research project focused on polyaniline reference electrode stability in cochlear fluid of the inner ear.

\section{Honors and Awards}

March 2016: Second Place E.J. Van Liere Memorial Convocation \& HSC Research Day basic science oral presentation

February 2015: First Place E. J. Van Liere Memorial Convocation \& HSC Research Day basic science poster presentation

May 2014: STEM Mountains of Excellence Scholarship for 2014-2015 academic year

February 2014: Second Place E. J. Van Liere Memorial Convocation \& HSC Research Day basic science poster presentation

\section{Professional Society Memberships}

American Association for Cancer Research (AACR) (March 2016-present) American Society for Cell Biology (ASCB) (November 2015-present)

\section{Scientific Presentations}

\section{Oral Presentations}

March 2016: E.J. Van Liere Memorial Convocation \& HSC Research Day

Poster Presentations

November 2016: Cancer Biology Training Consortium (CABTRAC) Annual Meeting

April 2016: American Association for Cancer Research (AACR) Annual Meeting March 2016: E.J. Van Liere Memorial Convocation \& HSC Research Day December 2015: The American Society for Cell Biology (ASCB) Annual Meeting February 2015: E.J. Van Liere Memorial Convocation \& HSC Research Day February 2014: E.J. Van Liere Memorial Convocation \& HSC Research Day October 2013: $2^{\text {nd }}$ Annual Appalachian Regional Cell Conference October 2012: $1^{\text {st }}$ Annual Appalachian Regional Cell Conference March 2012: E.J. Van Liere Memorial Convocation \& HSC Research Day 


\section{Teaching Experience}

Spring 2014: Immunology \& Medical Microbiology Colloquium, Teaching Assistant Fall 2013: Cellular Methods, Lecturer \& Student Course Coordinator

Fall 2012: Cellular Methods, Lecturer \& Student Course Coordinator

Fall 2008 - Spring 2009: General Chemistry Lab I, Teaching Assistant

Spring 2008: Accelerated Chemistry Lab II, Teaching Assistant

Fall 2007: General Chemistry Lab I, Teaching Assistant

\section{Registered Student Organization Memberships}

Spring 2014 - Spring 2015: WVU Health Science Center Student Advisory Board

Summer 2012 - Fall 2013: Planning Committee, $1^{\text {st }} \& 2^{\text {nd }}$ Annual Appalachian

Regional Cell Conference

Spring 2011-current: member of WVU Cell Biology Training Program

\section{Research Interests}

Cancer biology, invasion, metastasis, clinical intervention, pharmaceutical development, translational medicine, animal model systems

\section{Relevant Biology-Related Coursework}

Undergraduate

General Chemistry

Cellular Biology

Lab Safety Fundamentals

Endocrinology
Organic Chemistry

Inorganic Chemistry

Microbiology

Physiology

\section{Graduate}

Cell Biology

Cellular Methods

Molecular Genetics

Drug Discover \& Development

Oncogenes \& Signaling Networks

Cancer Pathology \& Therapeutics
Integrative Systems Biology

Scientific Integrity

Intro Cancer Cell Biology

Special Topics in Cancer Biology

Teaching Practicum

Teaching in Higher Education

\section{Bibliography}

Markwell, S.M., Ammer, A.G., Interval, E.T., Allen, J.L., Papenberg, B.W., Hames, R.A., Castaño, J.E., Schafer, D.A., Weed, S.A. Cortactin Phosphorylation by CK2 Regulates Arp2/3 Activity, Invadopodia Function and Tumor Cell Invasion. Mol Cancer Res 2019.

In Press 
Malcolm D. Mattes, Elizabeth Swart, Steven M. Markwell, Sijin Wen, and Linda C. Vona-Davis. Methods of Academic Course Planning for Cancer Biology PhD Students to Enhance Knowledge of Clinical Oncology. Cancer Res September 152017 (77) (18) 4741-4744; doi:10.1158/0008-5472.

Markwell, S.M.; Weed, S.A. Tumor and Stromal-Based Contributions to Head and Neck Squamous Cell Carcinoma Invasion. Cancers 2015, 7, 382-406.

Martin, K. H., Hayes, K. E., Walk, E. L., Ammer, A. G., Markwell, S. M., Weed, S. A. Quantitative Measurement of Invadopodia-mediated Extracellular Matrix Proteolysis in Single and Multicellular Contexts. J. Vis. Exp. (66), e4119, doi:10.3791/4119 (2012). 